\title{
LUDICS WITH REPETITIONS (EXPONENTIALS, INTERACTIVE TYPES AND COMPLETENESS) *
}

\author{
MICHELE BASALDELLA $^{a}$ AND CLAUDIA FAGGIAN $^{b}$ \\ ${ }^{a}$ Research Institute for Mathematical Sciences, Kyoto University, Kitashirakawa Oiwakecho, Sakyo- \\ ku, Kyoto 606-8502, Japan. \\ e-mail address: mbasalde@kurims.kyoto-u.ac.jp \\ ${ }^{b}$ Équipe PPS, CNRS and Université Paris 7, 2 place Jussieu, case 7017, 75251 Paris cedex 05, \\ France. \\ e-mail address: faggian@pps.jussieu.fr
}

\begin{abstract}
Ludics is peculiar in the panorama of game semantics: we first have the definition of interaction-composition and then we have semantical types, as a set of strategies which "behave well" and react in the same way to a set of tests. The semantical types which are interpretations of logical formulas enjoy a fundamental property, called internal completeness, which characterizes ludics and sets it apart also from realizability. Internal completeness entails standard full completeness as a consequence.

A growing body of work start to explore the potential of this specific interactive approach. However, ludics has some limitations, which are consequence of the fact that in the original formulation, strategies are abstractions of MALL proofs. On one side, no repetitions are allowed. On the other side, the proofs tend to rely on the very specific properties of the MALL proof-like strategies, making it difficult to transfer the approach to semantical types into different settings.

In this paper, we provide an extension of ludics which allows repetitions and show that one can still have interactive types and internal completeness. From this, we obtain full completeness w.r.t. a polarized version of MELL. In our extension, we use less properties than in the original formulation, which we believe is of independent interest. We hope this may open the way to applications of ludics approach to larger domains and different settings.
\end{abstract}

\section{INTRODUCTION}

Ludics is a research program started by Girard [22] with the aim of providing a foundation for logic based on interaction. It can be seen as a form of game semantics where first we have the definition of interaction (equivalently called composition, normalization), and then we have semantical types, as sets of strategies which "behave well" with respect to composition.

1998 ACM Subject Classification: F.4.1 Mathematical Logic (Proof theory), F.3 Logics and Meanings of Programs.

Key words and phrases: linear logic, ludics, game semantics, internal completeness.

* This paper is a completely revised and extended version of [5]. 
This role of interaction in the definition of types is where lies the specificity of ludics in the panorama of game semantics.

Recently, a growing body of work is starting to explore and to develop the potential of this specific approach, and to put at work the more general notion of type offered by ludics: the notion of type defined through interaction. We mention in particular work by Saurin on interactive proof-search as a logic programming paradigm [38, and work by Terui on computability [39]. Terui gives an especially interesting use of the notion of orthogonality ("to interact well"): if the strategy $\mathcal{D}$ describes an automaton, $\{\mathcal{D}\}^{\perp}$ (the set of all strategies which "interact well" with it) is the language accepted by that automaton. In [6] Basaldella and Terui have studied the traditional logical duality between proofs and models in the setting of computational ludics [39] enriched with exponentials (following our approach to exponentials [5], this paper). Both proofs and models live in an homogeneous setting, both are strategies, which are related by orthogonality. Finally, we observe that interactive types seem to be very natural also in process calculi; a bridge between process calculi and ludics has already been established in Faggian and Piccolo [15, which shows a close correspondence between the strategies of ludics and the terms of the linear $\pi$-calculus [40] - from this one can hope to transfer the whole approach of ludics to that setting.

There are also other lines of work in the literature which use orthogonality to define semantical types. We mention work by Pitts on parametricity [37, work by Krivine on realizability [28], work by Hyland and Schalk on categorical models of linear logic [27, work by Melliès and Vouillon on recursive types [34] and work by Paolini on parametric $\lambda$-calculus [36].

Interactive types. The computational objects of ludics - designs - can be seen as a linear form of Hyland-Ong (HO) innocent strategies (as shown in [14]) or as Curien's abstract Böhm trees [8, 11].

However, in game semantics, we first define the types (arenas, games), and then strategies on a given type. The type information guarantees that strategies compose well. In ludics, strategies are untyped, in the sense that all strategies are given on a universal arena (the arena of all possible moves); strategies can always interact with each other, and the interaction may terminate well (the two strategies "accept each other", and are said to be orthogonal) or not (they deadlock). An interactive type is a set of strategies which "compose well", and reacts in the same way to a set of tests (see Section 4). More concretely, a semantical type $\mathbf{G}$ is any set of strategies which reacts well to the same set of tests $\mathbf{E}$, which are themselves strategies (counter-strategies), that is $\mathbf{G}=\mathbf{E}^{\perp}$.

Internal completeness. With ludics, Girard also introduces a new notion of completeness, which is called internal completeness (see Section (5). This is a key - really defining element of ludics. We have already said that a semantical type is a set of strategies closed by biorthogonal $\left(\mathbf{G}=\mathbf{G}^{\perp \perp}\right.$ ). Internal completeness (in [22]) is the property which essentially says that the constructions on semantical types do not require any closure operation, i.e., they are already complete.

For instance, in [22] the interpretation of $A \oplus B$ (where $\oplus$ denotes the additive disjunction of linear logic) is defined as $(\mathbf{A} \cup \mathbf{B})^{\perp \perp}$. This set of terms could be in general strictly greater than $\mathbf{A} \cup \mathbf{B}$. But under certain conditions, it is possible to prove that $\mathbf{A} \cup \mathbf{B}$ is equal to $(\mathbf{A} \cup \mathbf{B})^{\perp \perp}$, and since the closure by biorthogonal does not introduce new terms, $\mathbf{A} \cup \mathbf{B}$ 
already gives a simple and complete description of what inhabits the semantical type, i.e., we have internal completeness.

While it is standard in realizability that a semantical type is a set $\mathbf{S}$ of terms closed by biorthogonal $\left(\mathbf{S}=\mathbf{S}^{\perp \perp}\right.$ ), when interpreting types one has to perform some kind of closure, and this operation might introduce new terms. Such new terms do not pose any essential problem when we are only interested in proving the soundness of a calculus w.r.t. a realizability model, that is, roughly, the property which states that if $\pi$ is a proof of a formula $A$, then we can construct from $\pi$ a strategy $\mathcal{D}$ which realizes the semantical interpretation $\mathbf{A}$ of $A$, i.e., $\mathcal{D} \in \mathbf{A}$. However, introducing new terms does make a difference when we are also interested in the other direction, namely (full) completeness: given a "good" strategy $\mathcal{D} \in \mathbf{A}$, we want to effectively associate to $\mathcal{D}$ a proof $\pi$ of $A$. Now the new terms which might have been introduced by the closure operation have to be taken into account. What internal completeness guarantees, is that we actually have a complete description of all the terms of a semantical types which correspond to logical proofs (or at least a subset which includes the "good" strategies).

In Girard's paper on ludics, the semantical types which are interpretations of propositional formulas enjoy internal completeness. This is really the key property (and the one used in [38, 39]). Full completeness (for multiplicative-additive-linear logic MALL, in the case of [22]) directly follows from it.

1.1. Contributions of the paper. The purpose of this paper is two-fold.

On the one hand, we show that it is possible to overcome the main limitation of ludics, namely the constraint of linearity, hence the lack of exponentials: we show that internal completeness (and from that full completeness) can be obtained also when having repetitions, if one extends in a rather natural way the setting of ludics.

On the other hand, we provide proofs which make use of less properties than the original ones given by Girard. Not only we do believe this improves the understanding of the results, but - more fundamentally - we hope this opens the way to the application of the approach of ludics to a larger domain.

We now give more details on the content of the paper.

1.1.1. Ludics architecture. A difficulty in [22] is that there is a huge amount of structure (see the "analytical theorems"), without a clear distinction between what are properties observed in the specific objects, and what is necessary to the construction. Strategies are an abstraction of MALL proofs, and enjoy many good properties. In [22], all proofs of the high level structure of ludics make essential use of these properties. Since some of those properties are very specific to the particular nature of the objects, this makes it difficult in principle to extend the - very interesting — approach of ludics to a different setting, or build the interactive types on different computational objects.

Ludics, as introduced in [22], is composed of several layers.

- At the low level, there is the definition of the untyped computational structures (strategies, there called designs) and their dynamics (interaction). Interaction allows the definition of orthogonality.

- The computational objects satisfy certain remarkable properties, called analytical theorems, in particular separation property, the ludics analogue of Böhm theorem for 
$\lambda$-calculus: two strategies $\mathcal{A}, \mathcal{B}$ are syntactically equal if and only if they are observationally equal (i.e., for any counter-strategy $\mathcal{C}$, the strategies $\mathcal{A}, \mathcal{B}$ react in the same way to $\mathcal{C})$.

- At the high level, there is the definition of interactive types, which satisfy internal completeness.

By relying on less structure, we show that the high level architecture of ludics is somehow independent from the low level entities (strategies), and in fact could be built on other - more general - computational objects.

In particular, separation is a strong property. It is a great property, but it is not a common one to have. However, the fact that computational objects do not enjoy separation does not mean that it is not possible to build the "high level architecture" of ludics. In fact, we show (Section 5) that the proofs of internal and full completeness rely on much less structure, namely operational properties of the interaction.

We believe that discriminating between internal completeness and the properties which are specific to the objects is important both to improve understanding of the results, and to make it possible to build the same construction on different entities.

In particular, strategies with repetitions have weaker properties with respect to the original - linear - ones. We show that it is still possible to have interactive types, internal completeness, and from this full completeness for a polarized version of the constant-only fragment of MELL (multiplicative-exponential-linear logic) that we call MELLS (Section 2).

The reason we restrict our attention to the constant-only fragment is that the treatment of propositional variables is rather complicated in ludics (see [22] and also [13]) and not strictly related to our purposes: the analysis of ludics with repetitions of actions. On the other hand, the extension of our framework to additives is straightforward.

1.1.2. Exponentials in ludics. The treatment of exponentials has been the main open problem in ludics since [22]. Maurel [32] has been the first one to propose a solution (a summary of this solution can also be found in [11, 23]). The focus of Maurel's work is to recover a form of separation when having repetitions; for this purpose, he develops a sophisticated setting, which is based on the use of probabilistic strategies: two probabilistic strategies "compose well" with a certain probability. This approach is however limited by its technical complexity; this is the main obstacle which stops Maurel from going further, and studying interpretation and full completeness issues.

In this work, we do not analyze the issue of separation, while we focus exactly into interactive types and internal completeness, and develop a fully complete interpretation from it.

Maurel also explores a simpler solution in order to introduce exponentials, but he does not pursue it further because of the failure of the separation property. Our work starts from an analysis of this simpler solution, and builds on it.

1.1.3. Our approach. In the literature, there are two standard branches of game semantics which have been extensively used to build denotational models of various fragments of linear logic. On the one hand, we have Abramsky-Jagadeesan-Malacaria style game semantics (AJM) [2] which is essentially inspired by Girard's geometry of interaction [18]. On the other hand, we have Hyland-Ong style game semantics (HO) [26], introducing innocent 
strategies. The main difference between those two game models is how the semantical structures corresponding to exponential modalities are built. In AJM, given a game $A$, $! A$ is treated as an infinite tensor product of $A$, where each copy of $A$ receives a different labeling index. Two strategies in !A which only differ by a different labeling of moves are identified. By contrast, in $\mathrm{HO}$ the notion of justification pointer substitutes that of index. The games $A$ and $! A$ share the same arena. Informally, a strategy in $! A$ is a kind of "juxtaposition" of strategies of $A$ such that by following the pointer structure, we can unambiguously decompose it as a set of strategies of $A$.

Girard's designs [22] are a linear form of HO innocent strategies [14]. Hence, the most natural solution to extend ludics to the exponentials is to consider as strategies, standard HO innocent strategies (on an universal arena). But in order to do so, there is a new kind of difficulty, which we deal with in this paper: we need to have enough tests.

More precisely, as we illustrate in Section 6, we need non-uniform counter-strategies. We implement and concretely realize this idea of non-uniform (non-deterministic) tests by introducing a non-deterministic sum of strategies, which builds on and refines work by Faggian and Piccolo [16]. More precise motivations and a sketch of the solution are detailed in Section 6.4

1.2. Plan of the paper. In Section 2, we introduce the polarized fragment of linear logic MELLS for which we will show a fully complete model in Section 11.

In Section 3, we recall the basic notions of HO innocent game semantics, which we then use in Section 4 to present Girard's ludics.

In Section 5 we review the results of internal completeness for linear strategies and outline a direct proof of full completeness.

In Section 6, we provide the motivations and an informal description of non-uniform strategies, and in Section 7 we give the formal constructions.

In Section 8 we describe in detail the composition of non-uniform strategies and in Section 9 we revise the notion of orthogonality in the non-uniform setting.

In Section 10 we introduce semantical types for MELLS, and we extend internal completeness to non-linear strategies. Full completeness is developed in Section 11.

In Section 12 we discuss related work and conclude the paper.

\section{Calculus}

We start by introducing a calculus that we call MELLS, which will be our "underlying" syntax; in Section 11, we prove that our model is fully complete for this calculus.

MELLS is a polarized variant of the constant-only propositional fragment of multiplicative-exponential linear logic MELL [17] based on synthetic connectives [21. Polarization, which we discuss in Section 2.2. is fundamental to Girard's computational analysis of classical logic [19] in which the system $\mathbf{L C}$ was introduced, and more recently to the design of Laurent's polarized linear logic LLP [29, 30]. 
2.1. MELL. Formulas of the constant-only, propositional, multiplicative-exponential linear logic MELL [17] are finitely generated by the following grammar:

$$
F::=\mathrm{I}|\perp| F \otimes F \mid F \text { \& } F|! F| ? F \text {. }
$$

The involutive linear negation $\perp$ is defined as follows:

$$
\mathrm{I}^{\perp}:=\perp ; \quad F \otimes G^{\perp}:=F^{\perp} \not \gamma G^{\perp} ; \quad ! F^{\perp}:=?\left(F^{\perp}\right) .
$$

A sequent, written $\vdash \Gamma$, consists of a (possibly empty) multi-set of formulas $\Gamma=$ $F_{1}, \ldots, F_{n}$. Given two multi-sets $\Gamma$ and $\Delta$ the expression $\Gamma, \Delta$ denotes their multi-set union. Given a multi-set $\Gamma=F_{1}, \ldots, F_{n}$ we write $? \Gamma$ for $? F_{1}, \ldots, ? F_{n}$.

Sequent calculus rules are given in Table 1,

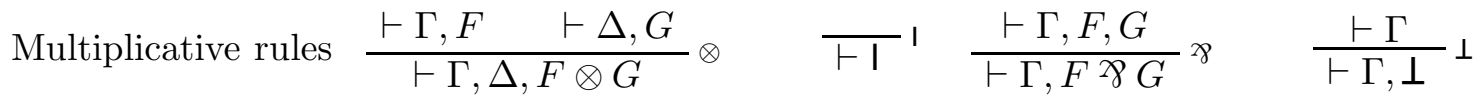

$$
\begin{aligned}
& \text { Exponential rules } \frac{\vdash ? \Gamma, F}{\vdash ? \Gamma, ! F} ! \quad \frac{\vdash \Gamma, F}{\vdash \Gamma, ? F} ? \quad \frac{\vdash \Gamma}{\vdash \Gamma, ? F} \mathrm{~W} \quad \frac{\vdash \Gamma, ? F, ? F}{\vdash \Gamma, ? F} \mathrm{C} \\
& \text { Cut-rule } \quad \frac{\vdash \Gamma, F \quad \vdash \Delta, F^{\perp}}{\vdash \Gamma, \Delta} \text { Cut }
\end{aligned}
$$

Table 1: MELL

A key feature of linear logic is the distinction between:

- linear formulas: I, $\perp, F \otimes F, F$ \& $F$;

- exponential formulas: ?F,!F.

Linear formulas can only be used once, while the modalities !,? allow formulas and sequent calculus derivations to be erased or duplicated in the sense we now make precise.

The possibility of discarding formulas is expressed in the sequent calculus by the weakening rule (or erase, in the bottom-up reading of a derivation) on ?F formulas:

$$
\frac{\vdash \Gamma}{\vdash \Gamma, ? F} \mathrm{w}
$$

The possibility of repeating formulas is taken into account by the contraction rule (or duplication, in the bottom-up reading) on ?F formulas:

$$
\frac{\vdash \Gamma, ? F, ? F}{\vdash \Gamma, ? F} \mathrm{C}
$$

In a dual sense, the modality ! allows derivations to be erased or duplicated during cutelimination procedure. Namely, (recall that $? F^{\perp}$ is defined as $!\left(F^{\perp}\right)$ ) we have:

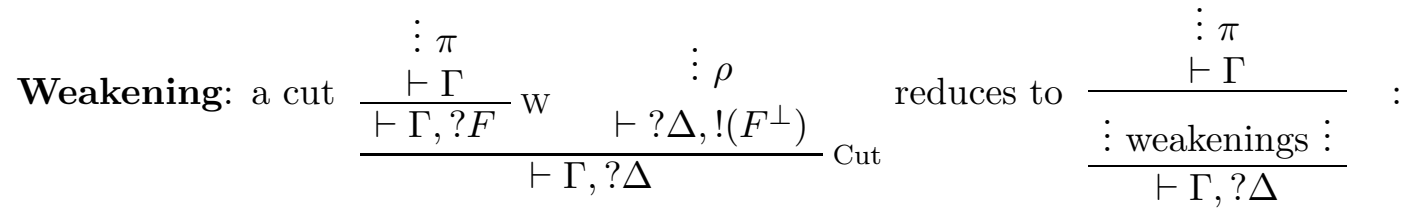

the derivation of $\vdash ? \Delta, !\left(F^{\perp}\right)$ is erased.

More important (for the purposes of this paper) is the case of contraction rule: 


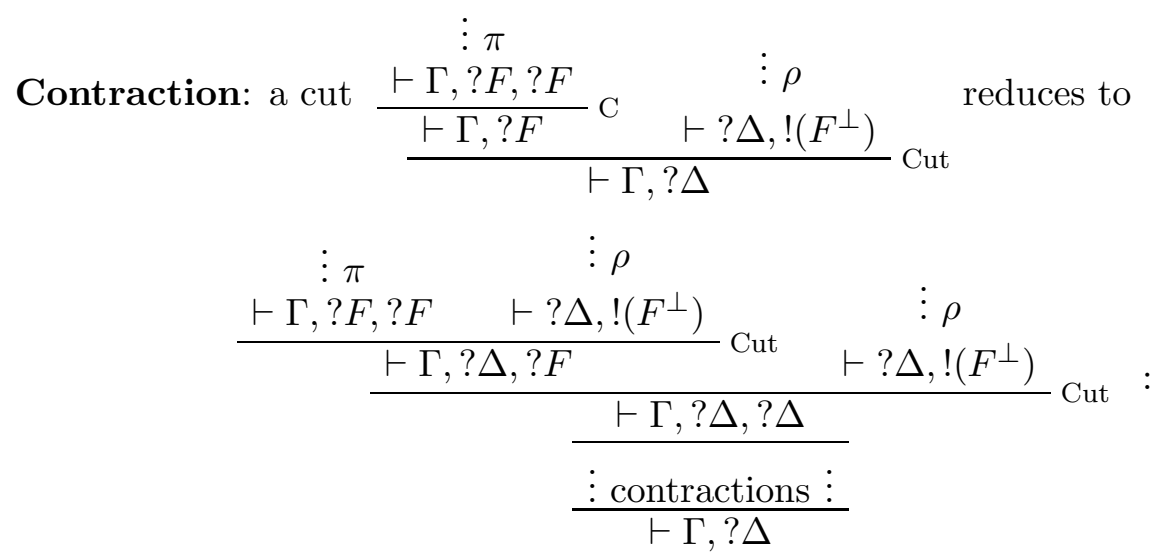

the derivation $\rho$ of $\vdash ? \Delta, !\left(F^{\perp}\right)$ can be used several times, once for each duplication of ?F.

2.2. Polarities, focalization and synthetic connectives. The connectives and constants of linear logic are split into two families according to their polarity (positive or negative). Let us discuss first the multiplicative-additive fragment MALL, where the distinction is clearly highlighted also typographically.

\section{Positive multiplicative : $\mathrm{I}, \otimes, \quad$ Positive additive : $\quad 0, \oplus$, \\ Negative multiplicative : $\perp, \not, \quad$ Negative additive : $T, \&$.}

The significance of polarities in linear logic was made explicit by Andreoli's seminal work on focalization [3]. The distinction into positive and negative corresponds in fact to properties of the connectives in proof construction [21, 9]. If a sequent is provable in linear logic, then it is provable with a proof which satisfies the proof-search strategy which we recall next (we recall that the polarity of a formula is the polarity of the outermost connective).

In the bottom-up construction of a proof:

(1) If there is a negative formula, keep on decomposing it until we get to atoms or positive subformulas.

(2) If there are not negative formulas, choose a positive formula, and keep on decomposing it until we get to atoms or negative subformulas.

For the exponential modalities ?,!, the situation is however a bit more complex (see e.g., 3 ] and [29]). This has lead Girard to analyze the exponentials via the following decomposition [20, 30]:

$$
! F=\downarrow \sharp F, \quad ? F=\uparrow b F,
$$

where $\sharp$ is the negative modality, $b$ is the positive modality, and $\downarrow, \uparrow$ are operators which change the polarity. The connectives $\sharp$ and $b$ are the "true" modalities, responsible of duplicative features. Hence, after the previous decomposition, the contraction rule would become:

$$
\frac{\vdash \Gamma, b P, b P}{\vdash \Gamma, b P} \mathrm{C}
$$

In this paper, we decided to use symbols which are more familiar, and we simply write $!_{N}$ (instead of $\sharp$ ) for the negative modality, and ? (instead of $b$ ) for the positive modality. 
Polarities allow us to have synthetic connectives [21, 23] i.e., maximal clusters of connectives of the same polarity. The key ingredient that allows for the definition of synthetic connectives is precisely the focalization property. In fact, from the point of view of logic, focalization (see the proof-search strategy above) means that each cluster of formulas with the same polarity can be introduced by a single logical rule (with several premises). By using synthetic connectives, formulas are in a canonical form, where immediate subformulas have opposite polarity. This means that in a (cut-free) proof, there is a positive/negative alternation of rules, which matches the standard Player (positive)/Opponent (negative) alternation of moves in a strategy (see Section 3 ).

2.3. MELLS. We now introduce in detail our calculus.

Formulas of MELLS split into positive $P$ and negative $N$ formulas, and they are inductively generated by the following grammar:

$$
\begin{array}{ccccc}
\text { Positive formulas : } & P & :=?_{P}\left(N_{1} \otimes \cdots \otimes N_{n}\right) & (n \geq 0) \\
\text { Negative formulas : } & N & :=!_{N}\left(P_{1} \not \cdots>P_{n}\right) & (n \geq 0)
\end{array}
$$

When $n=0$, we write $?_{P} \mathrm{I}$ and $!_{N} \perp$ for the positive and negative formula respectively. They are the only ground formulas of our calculus.

We will use $F$ as a variable for formulas and indicate the polarity also by writing $F^{+}$ or $F^{-}$. We use $P, Q, R, \ldots$ (resp. $N, M, L, \ldots$ ) for positive (resp. negative) formulas. To stress the immediate subformulas of some formula $F$, we often write $F^{+}\left(N_{1}, \ldots, N_{n}\right)$ and $F^{-}\left(P_{1}, \ldots, P_{n}\right)$.

The involutive linear negation ${ }^{\perp}$ is defined in the natural way:

$$
?_{P}\left(N_{1} \otimes \cdots \otimes N_{n}\right)^{\perp}:=!_{N}\left(N_{1}^{\perp} \text { ช } \ldots \text { ช } N_{n}^{\perp}\right) \text {. }
$$

In particular, $?_{P} \mathrm{I}^{\perp}=!_{N} \perp$.

A sequent of MELLS is a (possibly empty) multi-set of formulas $\Gamma$, written $\vdash \Gamma$, such that $\Gamma$ contains at most one (occurrence of) negative formula. In the sequel, $\Pi$ always stands for a (possibly empty) multi-set consisting of positive formulas only.

Rules of MELLS are given in Table 2 .

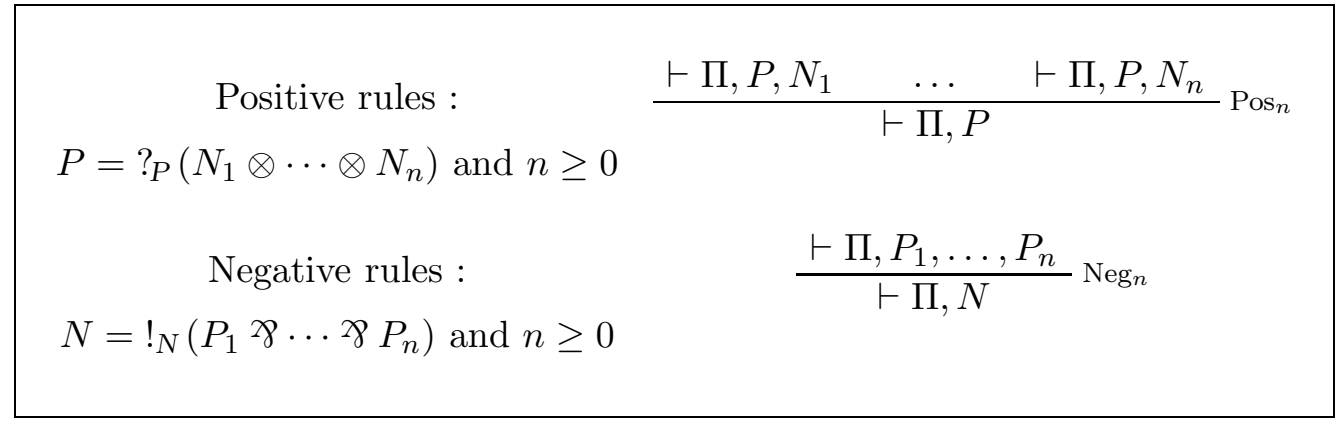

Table 2: MELLS

In particular, when $n=0$ (and hence $P=?_{P} \mathrm{I}$ and $N=!_{N} \perp$ ) we have:

$$
{\overline{\vdash \Pi, ?_{P} I}}^{\text {Pos } 0} \frac{\vdash \Pi}{\vdash \Pi, !_{N} \perp} \text { Neg }_{0}
$$


In the sequel we use variables $\pi, \rho, \psi, \theta \ldots$ for derivations of sequents in MELLS.

Structural rules (weakening and contraction) are on positive formulas only, and given implicitly in the positive rules.

2.4. Expressivity of MELLS. In Appendix $\mathrm{A}$ we discuss the expressivity of MELLS by relating it to more standard systems. In Appendix A.2 we give a correspondence between MELLS and a "focalized and synthesized" version of the $\neg, \wedge$ fragment of the sequent calculus for propositional intuitionistic logic LJ. In Appendix A.3 we relate MELLS to a more standard polarized version of MELL called MELL $_{\text {pol }}$ 29].

2.5. Cut-rule. The cut-rule for MELLS is the following one:

$$
\frac{\vdash \Xi, \Pi, P \quad \vdash \Delta, P^{\perp}}{\vdash \Xi, \Pi, \Delta} \mathrm{Cut}
$$

where the multi-set $\Xi$ is either empty or it consists of exactly one (occurrence of) negative formula and $\Delta$ is a multi-set of positive formulas.

This cut is admissible in MELLS (Theorem A.3), i.e., it does not improve the derivability of sequents w.r.t. the cut-free formulation of MELLS. A proof of this fact is given in Appendix A.1.

\section{HO INNOCENT GAME SEMANTICS}

An innocent strategy [26] can be described either in terms of all possible interactions for the player - strategy as set of plays - or in a more compact way, which provides only the minimal information for Player to move - strategy as set of views (see e.g., [35, 24, 10]). It is standard that the two presentations are equivalent: from a play one can extract the views, and from the views one can calculate the play.

In this paper we use the "strategy as set of views" description. Our presentation of innocent strategies adapts to our needs the presentations by Harmer [25] and Laurent [30].

Before introducing the formal notions, let us use an image. A strategy tells the player how to respond to a counter-player move. The dialogue between two players - let us call them $P$ (Player) and $O$ (Opponent) — will produce an interaction (a play). The "universe of moves" which can be played is set by the arena. Each move belongs to only one of the players, hence there are $P$-moves and $O$-moves. For $P$, the moves which $P$ plays are positive (active, output), while the moves played by $O$ are negative (passive, input), to which $P$ has to respond.

Polarities. Let $\mathrm{Pol}:=\{+,-\}$ be the set of polarities: positive (for Player) and negative (for Opponent). We use the symbol $\epsilon$ as a variable to range over polarities. 
Arenas. An arena is given by a directed acyclic graph, d.a.g. for short, which describes a dependency relation between moves and a polarity function, which assigns a polarity to the moves.

Definition 3.1 (Arena). An arena $A=\left(M_{A}, \vdash_{A}, \lambda_{A}\right)$ is given by:

- a directed acyclic graph $\left(M_{A}, \vdash_{A}\right)$ where:

- $M_{A}$ (nodes of the d.a.g.) is the set of moves;

$-\vdash_{A}$ (edges of the d.a.g.) is a well founded, binary enabling relation on $A$. If there is an edge from $m$ to $n$, we write $m \vdash_{A} n$. We call initial each move $m$ such that no other move enables it, and we write this as $\vdash_{A} n$.

- a function $\lambda_{A}: M_{A} \rightarrow P o l$ which labels each element with a polarity $\epsilon$.

Enabling relation and polarity have to satisfy the following property of alternation:

if $n \vdash_{A} m$, they have opposite polarity.

A non-empty arena whose initial moves have all the same polarity $\epsilon$, is said to be polarized [30]. In such a case, if $\epsilon$ is positive (resp. negative), we say that the arena is positive (resp. negative).

With a slight abuse of notation, we will write $m \in A$ for $m \in M_{A}$.

Strategies. A pointing string over a set $X$ is a string $s \in X^{*}$ with pointers between the occurrences of $s=s_{1} \ldots s_{n}$ such that, if $s_{i}$ points to $s_{j}$ then $j<i$, i.e., pointers always point back to earlier occurrences, and we have at most one pointer from any given occurrence of $s_{i}$.

Definition 3.2 (Justified sequence). Let $A$ be an arena. A justified sequence $s$ on $A$ is a pointing string $s=s_{1} \ldots s_{n} \in A^{*}$ which satisfies the following properties.

- Justification. For each non-initial move $s_{i}$ of $s$, there is a unique pointer to an earlier occurrence of move $s_{j}$, called the justifier of $s_{i}$, such that $s_{j} \vdash_{A} s_{i}$.

The polarity of a move in a justified sequence is given by the arena. We sometimes put in evidence the polarity of a move $x$ by writing $x^{+}$or $x^{-}$.

Definition 3.3 (View). A view on $A$ is a justified sequence on $A$ which satisfies:

- Alternation. No two following moves have the same polarity.

- View. If $s=s_{1} \ldots s_{n}$, for each negative (Opponent) move $s_{i}$ such that $i>1, s_{i}$ is justified by its immediate predecessor $s_{i-1}$.

Definition 3.4 (Strategy). A strategy $\mathcal{D}$ on $A$, denoted by $\mathcal{D}: A$ is a prefix-closed set of non-empty views, such that:

(1) Coherence. If $s . m, s . n \in \mathcal{D}$ and $m \neq n$ then $m, n$ are negative.

(2) Positivity. If $s . m$ is maximal in $\mathcal{D}$ (i.e., no other view extends it), then $m$ is positive.

In case that the arena is polarized - which is the case for all arenas we deal with in this paper - we call positive (resp. negative) a strategy on a positive (resp. negative) arena.

Remark 3.5. The choice of defining a strategy as a set of non-empty views is not standard, but is coherent with the setting of ludics. Following [22], a strategy can be an empty set (of views), whereas it never contains the empty view. 
As a consequence, condition (1) (Coherence) in Definition 3.4 implies that in a positive strategy, all views have the same first move. On the other hand, a negative strategy may also be a forest, rather than a tree (see Section 3.3).

3.1. Constructions on arenas. We give some constructions on arenas, which we will need in Section 4. $\emptyset)$.

Let $A_{1}, A_{2}$ be positive arenas such that the sets of moves are disjoint (i.e., $M_{A_{1}} \cap M_{A_{2}}=$ The arena $A_{1} \| A_{2}$ is defined as follows:

- $M_{A_{1} \| A_{2}}:=M_{A_{1}} \cup M_{A_{2}}$;

- $\lambda_{A_{1} \| A_{2}}(m):=\lambda_{A_{1}}(m)$ if $m \in M_{A_{1}}, \quad \lambda_{A_{1} \| A_{2}}(m):=\lambda_{A_{2}}(m)$ if $m \in M_{A_{2}}$;

- $\vdash_{A_{1} \| A_{2}}:=\vdash_{A_{1}} \cup \vdash_{A_{2}}$.

Observe that the structure of $A_{1} \| A_{2}$ is inherited from its constituent arenas; we place the arenas side-by-side. The construction immediately generalizes to the $n$-ary case: if $A_{1} \ldots, A_{n}(n \geq 1)$ are positive arenas such that for any $1 \leq i, j \leq n$ the sets of moves of $A_{i}$ and $A_{j}$ are pairwise disjoint, we obtain $A_{1}\|\cdots\| A_{n}$. It is immediate that $A_{1}\|\cdots\| A_{n}$ is a positive arena.

Let $C$ be a negative and $A$ be a positive arena, with disjoint sets of moves.

The arena $C \triangleleft A$ is defined as follows.

- $M_{C \triangleleft A}:=M_{C} \cup M_{A}$;

- $\lambda_{C \triangleleft A}(m):=\lambda_{C}(m)$ if $m \in M_{C}, \quad \lambda_{C \triangleleft A}(m):=\lambda_{A}(m)$ if $m \in M_{A}$;

- $m \vdash_{C \triangleleft A} n$ holds if

$m \vdash_{C} n$, or

$m \vdash_{A} n$, or

$m$ is initial in $C$ and $n$ is initial in $A$.

In words, the arenas $C$ is lifted on top of the roots of $A$, by adding each root of $A$ as child to each root of $C$.

3.2. Composition of strategies. Composition of strategies as sets of views has been studied in particular by Curien and Herbelin. They have introduced the View-AbstractMachine (VAM) [8, 11] by elaborating Coquand's Debates machine [7]. We will give more details in section 8 .

3.3. Conventions and notation. It is now convenient to fix some conventions and notation we will employ in all the rest of this paper.

We will often deal with sets of $n \geq 0$ elements, which we write $\left\{L_{i}: 1 \leq i \leq n\right\}$ or $\left\{L_{1}, \ldots, L_{n}\right\}$. The case $n=0$ always corresponds to the empty set.

Given $n \geq 0, I_{n}$ always stands for the set given as follows:

$$
I_{n}:= \begin{cases}\{1, \ldots, n\}, & \text { if } n>0 \\ \emptyset, & \text { if } n=0\end{cases}
$$


Given an index set $S$, we will often use (as in [22]) the notation $\left(L_{s}\right), s \in S$, to indicate a family of elements, indexed by $S$. When $S$ is clear from the context, we just write $\left(L_{s}\right)$.

Let $\mathcal{X}$ be a set of views. We call root each occurrence of move $a$ such that $a . s \in \mathcal{X}$.

Emphasizing the arborescent structure of a strategy, it is convenient to write a strategy with a single root $a$ as $\mathcal{D}=a . \mathcal{X}$, where $\mathcal{X}$ is the set of pointing strings $\{s: a . s \in \mathcal{X}\}$. Notice that $\mathcal{X}$ does not need to be a strategy. In case $\mathcal{X}$ is empty we just write $\mathcal{D}=a$.

We will come back on this in Section 4.1.2 and in Section 10.1, Lemma 10.1.

To better grasp the intuitions, we will draw strategies as trees whose nodes are labeled by moves. Nodes which are labeled by positive moves are circled.

Example 3.6. Let $\mathcal{D}$ be the strategy given by the closure under non-empty prefix of the set of views $\left\{a^{+} . b^{-} . d^{+}, a^{+} . c^{-} . e^{+}\right\}$(less formally, we could also write $\mathcal{D}=a^{+} .\left\{b^{-} . d^{+}, c^{-} . e^{+}\right\}$). We represent $\mathcal{D}$ by the following tree:

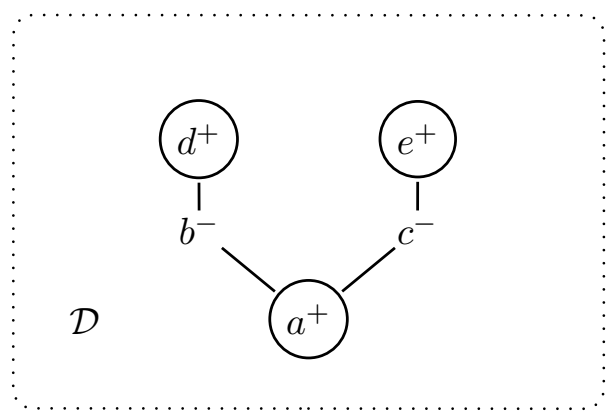

\section{LUdiCs, THE LINEAR CASE}

In this and next section we give a compact but complete presentation of ludics [22, introducing all definitions and technical results which are relevant to our approach, including internal completeness and full completeness. Our choice here is to give a presentation which fits into the language of game semantics.

Let us first stress again the peculiarity of ludics in the panorama of game semantics. In game semantics, one defines constructions on arenas which correspond to the interpretation of types. A strategy is always "typed", in the sense that it is a strategy on a specific arena: first we have the "semantical type" (the arena), and then the strategy on that arena. When strategies are opportunely typed, they interact (compose) well.

In the approach of ludics, there is only one arena (up to renaming): the universal arena of all possible moves. Strategies are "untyped", in the sense that all strategies are defined on the universal arena. Strategies then interact with each other, and the interaction can terminate well (the two strategies "accept" each other) or not (deadlock).

Two opposite strategies $\mathcal{D}, \mathcal{E}$ whose interaction terminates well, are said to be orthogonal, written $\mathcal{D} \perp \mathcal{E}$. Orthogonality allows us to define interactive types. A semantical type $\mathbf{G}$ is any set of strategies which react well to the same set of tests $\mathbf{E}$, which are themselves strategies (counter-strategies), that is $\mathbf{G}=\mathbf{E}^{\perp}$. 
Daimon. One of the goals in the program of ludics is to overcome the distinction between syntax (the formal system) on one side and semantics (its interpretation) on the other side. Rather then having two separate worlds, proofs are interpreted via proofs. To determine and test properties, a proof of $A$ should be tested with proofs of $A^{\perp}$. Ludics provides a setting in which proofs of $A$ interact with proofs of $A^{\perp}$; to this aim, it generalizes the notion of proof.

A proof should be thought in the sense of "proof-search" or "proof-construction": we start from the conclusion, and guess a last rule, then the rule above. What if we cannot apply any rule? A new rule is introduced, called daimon:

$$
\overline{\vdash \Pi} \dagger
$$

Such a rule allows us to assume any conclusion, or said in other words, it allows to close any open branch in the proof-search tree of a sequent.

In the semantics, the daimon is a special action which acts as a termination signal.

4.1. Strategies on a universal arena. We now introduce the notion of strategy as defined in ludics. The role of the arena becomes somehow secondary, while the central notion, is that of name. Strategies communicate on names. We can think of names as process algebras channels, which can be used to send outputs (if positive) or to receive inputs (if negative). Each strategy $\mathcal{D}$ will have an interface, which provides the names on which $\mathcal{D}$ can communicate with the rest of the world, and the use (input/output) of each name.

A name (called locus in [22]) is a string of natural numbers. We use the variables $\xi, \sigma, \alpha, \ldots$ to range over names. Two names are disjoint if neither is a prefix of the other one.

An action $x$ is either the symbol $\dagger$ (called daimon) or a pair $(\xi, I)$, where $\xi$ is a name, and $I$ is a finite subset of $\mathbb{N}$. In this paper, we will always assume $I$ of the form $I_{n}=\{1, \ldots, n\}$, for some $n \geq 0$ (see Section 3.3) 1 . Given an action $(\xi, I)$ on the name $\xi$, the set $I$ is actually a shortcut for the set of the names $\{\xi i: i \in I\}$ which are generated from $\xi$ by this action. We call proper an action of the form $(\xi, I)$ (to contrast with a $\dagger$ action, which has a different function). The role of proper actions and of the $\dagger$ actions will be very different when defining interaction between strategies.

The prefix relation (written $\xi \sqsubseteq \sigma$ ) induces a natural relation of dependency on names, and hence on the proper actions, which generates an arena.

Definition 4.1 (Universal arena (on a name)). Given a name $\xi$ and a polarity $\epsilon \in\{+,-\}$, the universal arena $A(\xi, \epsilon)$ is the tuple $(M, \vdash, \lambda)$ defined as follows:

(1) The set of moves $M$ is the set of all actions of the form $\left(\xi^{\prime}, I\right)$, where $\xi \sqsubseteq \xi^{\prime}$ and $I$ is a finite subset of $\mathbb{N}$.

(2) The polarity of the initial actions is $\lambda((\xi, I))=\epsilon$ for each $I$; the polarity of any other action is the one induced by alternation.

The enabling relation $x \vdash y$ is defined as follows:

\footnotetext{
${ }^{1}$ We can think of the set $I_{n}$ only just as an "arity provider." We use the notation $I_{n}$ first of all for compatibility (and comparability) with [22], in which the general case is needed to deal with the additives. Secondly, this choice leaves open the possibility to extend our work with the additive structure of ludics, without essential modifications.
} 


$$
x \vdash y \text { if } x=\left(\xi^{\prime}, I\right) \text { and } y=\left(\xi^{\prime} i, J\right) \text {, with } i \in I .
$$

The choice of a name "delocalizes" the universal arena on a specific name. Of course, all universal arenas are isomorphic up to the choice of some name $\xi$.

Definition 4.2 (Interface). An interface $\Gamma$ (called base in [22]) is a (possibly empty) finite set of pairwise disjoint names, together with a polarity for each name, such that at most one name is negative. If a name $\xi$ has polarity $\epsilon$, we write $\xi^{\epsilon} \in \Gamma$.

With an abuse of notation, in the sequel we often write $\Gamma=\xi_{1}^{\epsilon_{1}}, \ldots, \xi_{n}^{\epsilon_{n}}$ instead of $\Gamma=\left\{\xi_{1}^{\epsilon_{1}}, \ldots, \xi_{n}^{\epsilon_{n}}\right\}$. When no confusion arises, we also omit the polarities and simply write $\Gamma=\xi_{1}, \ldots, \xi_{n}$.

An interface $\Gamma$ is negative if it contains a negative name, positive otherwise. In particular, the empty interface is positive.

Definition 4.3 (Universal arena on an interface). We denote by $A_{\dagger}$ the arena whose set of moves is $\{\dagger\}$, with the polarity of $\dagger$ being positive.

Let $\Pi=\xi_{1}, \ldots, \xi_{n}$ be a (possibly empty) positive interface.

The universal arena on the interface $\Pi$ is the arena

$$
U(\Pi):=A\left(\xi_{1},+\right)\|\cdots\| A\left(\xi_{n},+\right) \| A_{\dagger} .
$$

The universal arena on a (negative) interface $\left\{\sigma^{-}\right\} \cup \Pi$ is the arena

$$
U(\Gamma):=A(\sigma,-) \triangleleft U(\Pi) \text {. }
$$

Remark 4.4. We observe that, according to Definition [3.1, a universal arena $U(\Gamma)$ is a polarized arena; the polarity of the arena is that of its initial actions, which results the same as the polarity of the interface $\Gamma$.

Example 4.5. The universal arena $U\left(\xi^{+}\right)$can be pictured as in Figure 1. An arrows from $b$ to $a$ stands for an enabling $a \vdash b$; the polarity of the actions is given as follows: actions lying on even (resp. odd) layers have positive (resp. negative) polarity.

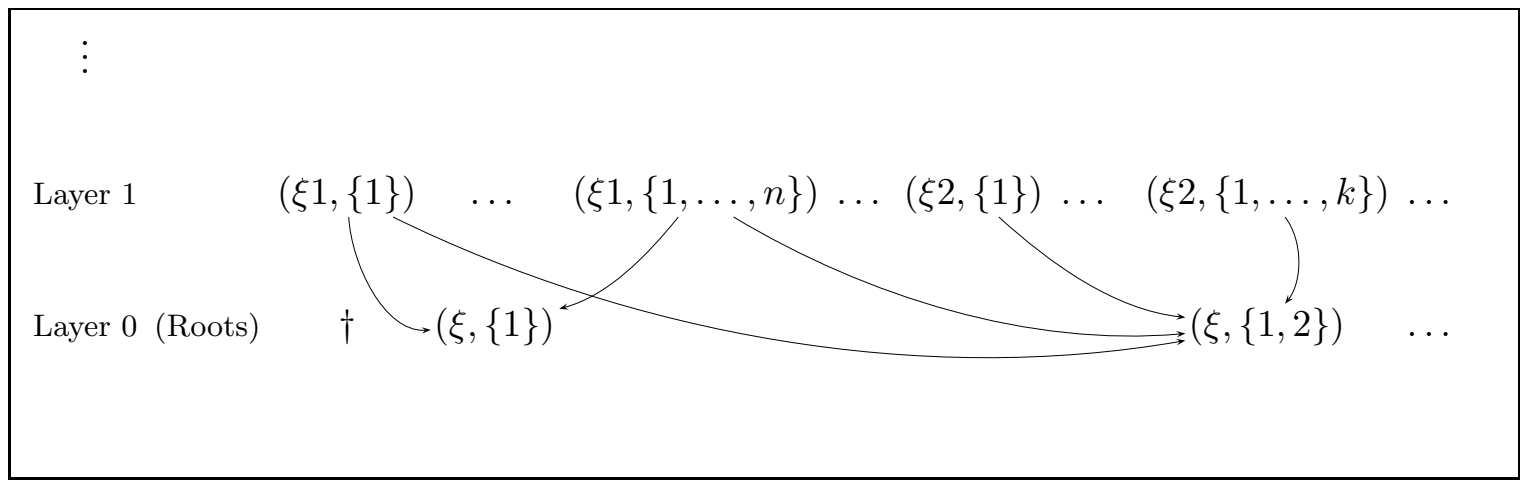

Figure 1: The universal arena $U\left(\xi^{+}\right)$

Definition 4.6 (Untyped strategies). Let $\Gamma$ be an interface. A strategy $\mathcal{D}$ on $\Gamma$, also written $\mathcal{D}: \Gamma$, is a strategy (in the sense of Definition 3.4) on the universal arena $U(\Gamma)$.

We point out that here we call strategies and views (following the language of game semantics) what in [22] is indicated as designs and chronicles respectively. 
Examples 4.7 (Basic strategies: $\mathfrak{D a i}, \mathfrak{F} \mathfrak{i} d, \emptyset$ ). Let us point out a few relevant strategies.

- There are two positive strategies which play a key role in ludics: $\mathfrak{D a i}$ and $\mathfrak{F i d}$. Both are defined on any positive interface, and in particular also on the empty interface. In fact, they are the only possible strategies on the empty interface.

- $\mathfrak{D a i}$ is the strategy which consists of only one action $\{\dagger\}$; it is called daimon.

- $\mathfrak{F} \mathfrak{i d}$ is the empty strategy; it is called faith.

- We highlight also a simple example of negative strategy: the empty strategy on a negative interface. We will denote this strategy simply by $\emptyset$.

\subsubsection{Totality.}

Definition 4.8 (Totality). We say that an untyped strategy is total when it is not $\mathfrak{F i d}$.

The definition of totality deserves some explanations. First, it is not the usual totality condition of game semantics. Totality, in ludics, is closely connected with the definition of orthogonality, hence it will become clear only after Section 4.3. Let us however anticipate some remarks, to justify why $\mathfrak{F i d}$ is not a total strategy (while the empty negative strategy $\emptyset$ is total). The definition of orthogonality is based on the fact that there are only two possible outcomes when we make interact two strategies on dual interfaces: either $\mathfrak{F i d}$ or $\mathfrak{D a i}$. The former is interpreted as failure of the interaction process (no output/deadlock), while $\mathfrak{D a \mathfrak { i }}$ is interpreted as success of the interaction process. In such a case, the strategies are said to be orthogonal. We will see (Example 4.27) that, for the way in which the interaction is defined, when we have a negative strategy $\mathcal{E}$, its interaction with $\mathfrak{D a \mathfrak { i }}$ always succeeds, while its interaction with $\mathfrak{F i d}$ always fails (i.e., there is no output).

Moreover, the fact that the interaction with $\mathfrak{D a \mathfrak { i }}$ succeeds for any negative strategy, including the case of the empty negative strategy allows us also to understand why the negative empty strategy is total. Such a strategy is in fact the smallest strategy to be orthogonal to $\mathfrak{D a i}$.

In terms of linear logic, the empty negative strategy $\emptyset$ interprets the rule for $T$, while $\mathfrak{D a \mathfrak { i }}$ would correspond to a "proof" of 0 (here $\mathrm{T}$ and 0 denote the additive constants top and zero respectively). But there is no corresponding "proof-object" for $\mathfrak{F i d}$. We want a strategy which corresponds to a proof to be total.

\subsubsection{Linearity.}

Definition 4.9 (Linearity). Given a strategy $\mathcal{D}: \Gamma$, we say that an occurrence of action $(\xi, I)$ in $\mathcal{D}$ is linear if the name $\xi$ is only used by that occurrence of action. We say that $\mathcal{D}: \Gamma$ is linear if each occurrence of (proper) action in $\mathcal{D}$ is linear.

Linearity has as consequence that all pointers are trivial (each move has only one possible justifier and the prefix relation between names univocally tells us which is), and then can be forgotten.

Remark 4.10. Linear strategies are essentially the strategies introduced in [22]. Our condition is actually more strict than the condition in [22], and allows us a simplification of some details, by restricting the setting only to multiplicatives. The linearity condition in [22] is slightly more complex, in order to take into account also the additive structures (additive duplication is allowed), but for our discussion it is enough to ask that in a strategy each name is only used once. 
Observe that a relevant consequence of our strict condition of linearity is that both positive and negative strategies have a single root (i.e., they are trees, and not forests). Hence, we can always write a negative strategy of root $x$ as $x^{-} . \mathcal{C}$. It is immediate to check that $\mathcal{C}$ is a positive strategy. As for positive strategies, let $\mathcal{D}$ have $\operatorname{root}\left(\sigma, I_{n}\right)^{+}$, i.e., $\mathcal{D}=\left(\sigma, I_{n}\right)^{+}$. $\mathcal{E}$. All views in $\mathcal{E}$ have a first action of the form $(\sigma i, K)$, with $i \in I_{n}$. We partition $\mathcal{E}$ into maximal subsets $\mathcal{E}_{i}, 1 \leq i \leq n$ of views which start with the same action. The linearity condition implies that each $\mathcal{E}_{i}$ is a negative strategy which either has a unique root of the form $(\sigma i, K)^{-}$or is the empty negative strategy $\left(i . e ., \mathcal{E}_{i}=\emptyset\right)$. We will also write $\mathcal{D}=\left(\sigma, I_{n}\right)^{+} .\left\{\mathcal{E}_{1}, \ldots, \mathcal{E}_{n}\right\}$, to emphasize the tree structure.

From now on, and till the end of Section 5, strategies are always linear strategies.

4.2. Dynamics in the linear case. The composition of untyped strategies can be described via the VAM machine (see Section 8). For the moment, we only describe normalization in the linear case (see [22, 12]). This case is simpler, but has all the key ingredients to follow most of the examples of this paper.

The central idea beyond names, is that we can compose two strategies when their interfaces have a common name, which appears in the two interfaces with opposite polarity. The key case is the following: $\mathcal{D}_{1}: \sigma^{+}, \Gamma$ and $\mathcal{D}_{2}: \sigma^{-}, \Delta$, with $\Gamma \cap \Delta=\emptyset$.

$\mathcal{D}_{1}$ can communicate with $\mathcal{D}_{2}$ through the name $\sigma$. The shared name $\sigma$ is called a cut.

Rather than define the dynamics for pairs of strategies, it is more convenient to define it for an arbitrary finite number of strategies at the same time.

Definition 4.11 (Cuts and cut-nets). Two interfaces $\Gamma_{1}, \Gamma_{2}$ are said to be compatible if all names are pairwise disjoint or equal, and in the latter case any name which appears in both the interfaces, appears with opposite polarity. If $\xi^{+} \in \Gamma_{i}$ and $\xi^{-} \in \Gamma_{j}$, the name $\xi$ is said to be a cut.

A non-empty finite set of interfaces $\left\{\Gamma_{1}, \ldots, \Gamma_{n}\right\}$ is said to be valid if:

- $\Gamma_{1}, \ldots, \Gamma_{n}$ are pairwise compatible interfaces;

- the graph defined here below is connected and acyclic:

- nodes: $\Gamma_{1}, \ldots, \Gamma_{n}$;

- edges: there is an edge between $\Gamma_{i}$ and $\Gamma_{j}$ for each cut $\xi$ such that $\xi^{+} \in \Gamma_{i}$ and $\xi^{-} \in \Gamma_{j}$. A non-empty finite set of strategies $\mathcal{R}=\left\{\mathcal{D}_{1}: \Gamma_{1}, \ldots, \mathcal{D}_{n}: \Gamma_{n}\right\}$ is said to be a cut-net if $\left\{\Gamma_{1}, \ldots, \Gamma_{n}\right\}$ is a valid set of interfaces.

We also write $\mathcal{R}=\left\{\mathcal{D}_{1}, \ldots, \mathcal{D}_{n}\right\}$ when the interfaces of the strategies are clear from the contexts or irrelevant for our purposes.

Remark 4.12. The acyclicity condition implies that any two interfaces in a valid set have at most one name in common. Observe that if $\left\{\Gamma_{1}, \Gamma_{2}\right\}$ is a valid set of interfaces, then there is exactly one cut (at most one by acyclicity, at least one by connectedness).

Example 4.13. The following three sets of interfaces are all pairwise compatible, but only the third one (3) is valid: (1) is not acyclic, an (2) is not connected. 2

\footnotetext{
${ }^{2}$ In the picture, we orient the edges for future uses of this example (Proposition 4.18). But recall that the edges of the graph as given in Definition 4.11 are not oriented.
} 

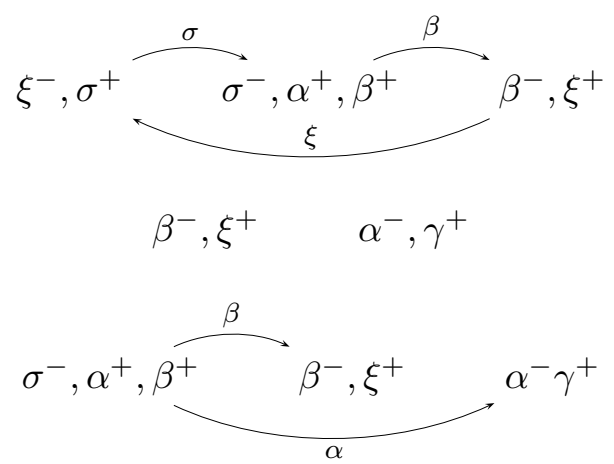

Definition 4.14 (Closed cut-net). A cut-net $\mathcal{R}$ is closed if all names in the interfaces are cuts.

Example 4.15. We have a typical example of closed cut-net when we have strategies on opposite interface, such as $\mathcal{D}: \xi^{+}$and $\mathcal{E}: \xi^{-}$.

Remark 4.16. Closed cut-nets contain exactly one positive strategy (see also Remark 4.19).

Given a cut-net $\mathcal{R}=\left\{\mathcal{D}_{1}: \Gamma_{1}, \ldots, \mathcal{D}_{n}: \Gamma_{n}\right\}$, the result of the composition is called normal form, and denoted by $\llbracket \mathcal{R} \rrbracket$. Composition (normalization) follows the standard paradigm of parallel composition (the interaction) plus hiding of internal communication: $\llbracket \mathcal{R} \rrbracket$ is obtained from the result of the interaction by hiding all the actions on internal names. The result is a strategy on the interface which is obtained from $\bigcup \Gamma_{i}$ by hiding all cut names.

Given the cut-net $\mathcal{R}$, if $\Theta$ is the set of the cuts, then $\left(\bigcup_{1 \leq i \leq n} \Gamma_{i}\right) \backslash \Theta$ is an interface, which is called the interface of $\mathcal{R}$.

Composition of the strategies in a cut-net can be described in several equivalent ways. The merging of orders [22, 16] is the most compact and mathematically pleasant — but it is specific to the linear case. In this paper, we prefer to describe composition via an abstract machine (the LAM [23, 12]), because this will serve as an introduction to the machine which performs the composition of strategies with repetitions.

Let us first introduce with a small example the basic ideas on the way in which strategies in a cut-net interact with each other to produce the normal form. Since each action appears only once, the dynamics is extremely simple: we match actions of opposite polarity.

Example 4.17. Let us consider the following strategies:

- $\mathcal{D}=a^{+} . b^{-} \cdot \dagger$;

- $\mathcal{E}=a^{+}$

- $\mathcal{F}=a^{-} . b^{+}$

where $a=(\xi, I)$ and $b=(\xi 1, K)$, so that $\mathcal{D}, \mathcal{E}: \xi^{+}$and $\mathcal{F}: \xi^{-}$. Notice that $b$ is enabled by $a$ in both the underlying universal arenas. We can draw these strategies as follows: 

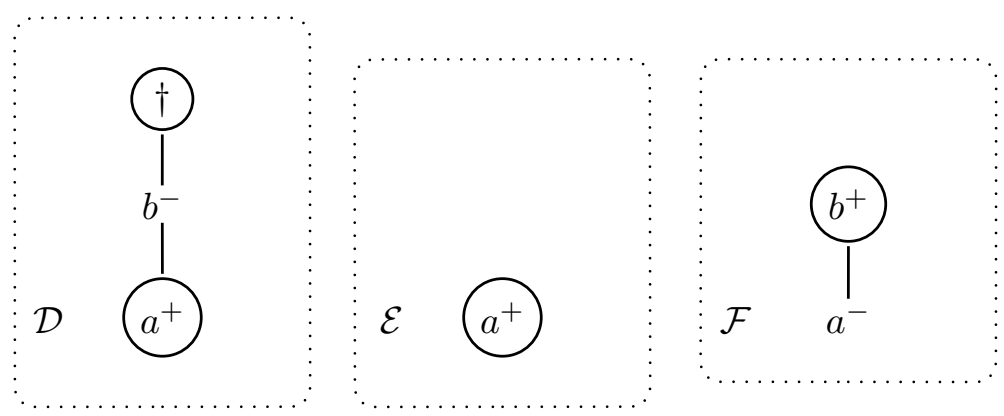

Let us have $\mathcal{D}$ interact with $\mathcal{F}$. Remember the intuition that "+" corresponds to an output, and "-" to an input. $\mathcal{D}$ starts by playing the move $a^{+}, \mathcal{F}$ receives $a$ and checks its answer to that move, which is $b^{+}$. If $\mathcal{D}$ receives input $b$, its answer is $\dagger$, which terminates the interaction. Summing up, the interaction produces $a^{+} \cdot a^{-} \cdot b^{+} \cdot b^{-} \cdot \dagger$. If we hide the internal communication, we get $\dagger$, i.e., $\llbracket \mathcal{D}, \mathcal{F} \rrbracket=\mathfrak{D} \mathfrak{a i}$.

If we have $\mathcal{E}$ interacting with $\mathcal{F}$, we again match $a^{+}$with $a^{-}$. Then $\mathcal{F}$ plays $b$, but $\mathcal{E}$ has not considered the action $b$. Here we have a deadlock i.e., $\llbracket \mathcal{E}, \mathcal{F} \rrbracket=\mathfrak{F i d}$.

4.2.1. Linear composition: LAM (Loci-Abstract-Machine). To formally define normalization, we need to introduce a notion of order on the strategies of a cut-net; such a definition makes explicit the order in which normalization accesses the strategies.

Up to the end of this section, let us fix a cut-net $\mathcal{R}=\left\{\mathcal{D}_{1}: \Gamma_{1}, \ldots, \mathcal{D}_{n}: \Gamma_{n}\right\}$.

Proposition 4.18 (Main strategy). Given a cut-net $\mathcal{R}$, we define the following precedence relation < on the strategies:

$\mathcal{D}_{i}: \Gamma_{i}<\mathcal{D}_{j}: \Gamma_{j}$ if there is a cut $\xi$ such that $\xi^{+} \in \Gamma_{i}$ and $\xi^{-} \in \Gamma_{j}$.

The order induced by this precedence relation has a minimal element, which is called the main strategy of $\mathcal{R}$.

Proof. Since the graph induced by the valid set of interfaces is acyclic (Definition 4.11), the order is arborescent, because each interface contains at most one negative name, hence each strategy has at most one immediate predecessor.

To verify that there is a unique minimal element, we observe that the precedence relation induces also an orientation on the edges of the graph: for each cut $\xi$ such that $\xi^{+} \in \Gamma_{i}$ and $\xi^{-} \in \Gamma_{j}$, the edge is oriented from $\Gamma_{i}$ to $\Gamma_{j}$ (see Example 4.13). Since the graph is connected, the oriented graph is a tree, and not a forest. Hence there is a unique minimal strategy.

Remark 4.19. In a cut-net, there is at most one positive strategy, which necessarily is the main one. The argument is the same as in the previous proof.

A cut name $\xi$ and all the names hereditarily generated from $\xi$, are said to be internal. We call internal an action on an internal name, otherwise the action is said to be visible.

Observe that if $\mathcal{R}$ is a closed cut-net, then all the names are internal (and its interface is empty). The only possible visible actions are $\dagger$ actions.

The following lemma is also an easy preliminary observation.

Lemma 4.20. Let $\mathcal{R}$ be a cut-net. Given a proper action $x^{\epsilon}$, it occurs in at most one $\mathcal{D} \in \mathcal{R}$. 
Definition 4.21 (LAM [12, 23]). Given a cut-net $\mathcal{R}$, the set $L A M(\mathcal{R})$ is the set of sequences of actions defined as follows.

(1) (Initialization) If the main strategy of $\mathcal{R}$ is empty, we set $\operatorname{LAM}(\mathcal{R}):=\emptyset$. Otherwise, if $a$ is the root of the main strategy, we set $a \in L A M(\mathcal{R})$.

(2) Let $p=x_{1} \ldots x_{n} \in L A M(\mathcal{R})$. We have the following two cases:

a. (Continuation)

The action $x_{n}$ is either a negative action or a positive visible action. If $x_{n}$ is proper, by the previous lemma there exists a unique strategy $\mathcal{D} \in \mathcal{R}$ such that a view $s . x_{n} \in \mathcal{D}$. In such a case, for each action $a$ which extends $s . x_{n}$ in $\mathcal{D}$ we set p. $a \in \operatorname{LAM}(\mathcal{R})$.

b. (Jump) The action $x_{n}=a^{+}$is an internal positive action. If there is $\mathcal{E} \in \mathcal{R}$ such that a view $s . a^{-} \in \mathcal{E}$, we set $p . a^{-} \in L A M(\mathcal{R})$. We say that $a^{-}$matches $a^{+}$.

Let us informally explain how the machine calculates the interaction of a cut-net $\mathcal{R}$. The machine visits actions of the strategies in $\mathcal{R}$ and collects the sequences of visited actions, proceeding as follows:

- We start on the root of the main strategy of a cut-net $\mathcal{R}$.

- If we visit a visible action $a$ occurring in some $\mathcal{D} \in \mathcal{R}$, we continue to explore the current strategy $\mathcal{D}$. The process branches when $a$ is a branching node of $\mathcal{D}$.

- If we visit an internal action $a^{+}$occurring in $\mathcal{D}$ we match it with its opposite $a^{-}$occurring in $\mathcal{E} \in \mathcal{R}$, then we continue to collect actions in $\mathcal{E}$ (this is a jump of the machine).

- We may eventually stop when either we reach a maximal action or an internal action which has no match.

Definition 4.22 (Hiding). Given a cut-net $\mathcal{R}$, and $p=x_{1}, \ldots, x_{n} \in L A M(\mathcal{R})$, we define $\operatorname{hide}(p)$ as the sequence obtained from $p$ by deleting all the internal actions.

Definition 4.23 (Normal form). Let $\mathcal{R}$ be a cut-net. We define the set $I(\mathcal{R})$ of the interactions of $\mathcal{R}$ as the closure by non-empty prefix of

$$
\{q . c \in L A M(\mathcal{R}): c \text { visible and positive }\} .
$$

The normal form of $\mathcal{R}$, denoted by $\llbracket \mathcal{R} \rrbracket$ is defined as

$$
\llbracket \mathcal{R} \rrbracket=\{\operatorname{hide}(p): p \in I(\mathcal{R}) \text { and hide }(p) \text { non-empty }\} .
$$

The normal form of a cut-net is a strategy [22, 9, 23].

Theorem 4.24. If $\mathcal{R}$ is a cut-net of interface $\Gamma$, then $\llbracket \mathcal{R} \rrbracket$ is a strategy on $\Gamma$.

Composition satisfies associativity, in the following sense [22, 9, 23].

Theorem 4.25 (Associativity). Let $\mathcal{R}$ be a cut-net which can be partitioned into cut-nets $\mathcal{R}=\mathcal{R}_{1} \cup \ldots \cup \mathcal{R}_{n}$. We have:

$$
\llbracket \mathcal{R} \rrbracket=\llbracket \llbracket \mathcal{R}_{1} \rrbracket, \ldots, \llbracket \mathcal{R}_{n} \rrbracket \rrbracket
$$


Remark 4.26. The standard associativity of game semantics (in terms of "morphisms") can be expressed in this setting, considering the universal arena on an interface of the form $\alpha^{-}, \beta^{+}$as an arena "of the form $A \rightarrow B$." Let us write for now $\alpha \rightarrow \beta$ instead of $\alpha^{-}, \beta^{+}$.

Let $\alpha, \beta, \gamma, \delta$ be disjoint names and consider $\mathcal{D}: \alpha \rightarrow \beta, \mathcal{E}: \beta \rightarrow \gamma$ and $\mathcal{F}: \gamma \rightarrow \delta$. By Theorems 4.24 and 4.25, we have that

$$
\llbracket \llbracket \mathcal{D}, \mathcal{E} \rrbracket, \mathcal{F} \rrbracket=\llbracket \mathcal{D}, \mathcal{E}, \mathcal{F} \rrbracket=\llbracket \mathcal{D}, \llbracket \mathcal{E}, \mathcal{F} \rrbracket \rrbracket
$$

is a strategy on interface $\alpha \rightarrow \delta$.

Example 4.27 (Interaction with $\mathfrak{D a \mathfrak { i }}$ or $\mathfrak{F} \mathfrak{i d}$ ). We can now examine how $\mathfrak{F i d}$ and $\mathfrak{D a \mathfrak { i }}$ behave in the normalization, and so make precise the discussion in Section 4.1.1. Let $\mathcal{R}=\left\{\mathcal{D}, \mathcal{E}_{1}, \ldots, \mathcal{E}_{n}\right\}$ be a positive cut-net, where $\mathcal{D}$ is the unique positive strategy (see Remark 4.19). Let us see what happens when $\mathcal{D}=\mathfrak{D} \mathfrak{a} \mathfrak{i}$ or $\mathcal{D}=\mathfrak{F i d}$.

- The interaction starts from $\mathcal{D}$ (because it is the main strategy).

- If $\mathcal{D}$ is $\mathfrak{D a i}$, the interaction reaches $\dagger$ at the first step, and terminates immediately, whatever the other (negative) strategies in $\mathcal{E}_{1}, \ldots, \mathcal{E}_{n}$ are.

- If $\mathcal{D}$ is $\mathfrak{F} \mathfrak{i d}$, nothing can happen. Since $\mathcal{D}$ is empty, the output of the interaction is also empty.

Summing up, we always have that:

$$
\llbracket \mathfrak{D a} \mathfrak{a}, \mathcal{E}_{1}, \ldots, \mathcal{E}_{n} \rrbracket=\mathfrak{D} \mathfrak{a} \mathfrak{i} \quad \text { and } \quad \llbracket \mathfrak{F i d}, \mathcal{E}_{1}, \ldots, \mathcal{E}_{n} \rrbracket=\mathfrak{F i d} .
$$

4.2.2. A notation to describe the interaction. In the sequel, given two strategies $\mathcal{D}$ and $\mathcal{F}$ we often describe their interaction in the following graphical way:

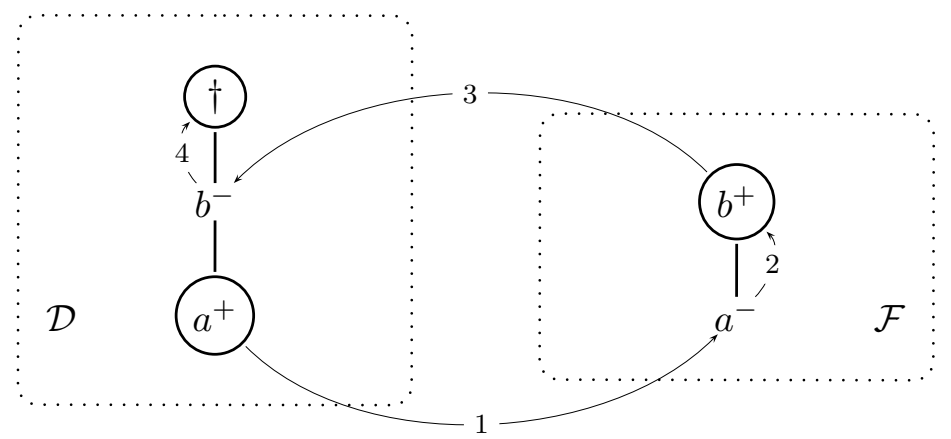

Here, we have taken $\mathcal{D}$ and $\mathcal{F}$ as in Example 4.17. We draw tagged arrows to denote the matching of actions (e.g., $a^{+}$matches $a^{-}$at step 1) and the (unique) positive action (the "answer") above a reached negative action (e.g.,,$b^{+}$after $a^{-}$). The tags $1,2, \ldots$ are only needed to record the chronological order in which actions are visited. Following the arrows with this order, we retrieve the sequence of actions $a^{+} \cdot a^{-} \cdot b^{+} . b^{-} \cdot \dagger$ which corresponds to the interaction of $\mathcal{D}$ and $\mathcal{F}$ of Example 4.17. 
4.3. Orthogonality. The most important case of composition in ludics is the closed case, the typical example being a cut-net given by two strategies on opposite interfaces $\mathcal{D}: \xi^{+}$ and $\mathcal{E}: \xi^{-}$.

We already observed that in this case, all names are internal, and the interface of the cut-net is empty. Since we know that there are only two possible strategies which have empty interface: $\mathfrak{F i d}$ and $\mathfrak{D a i}$, we only have two possible values as normal form. $\mathfrak{F i d}$ and $\mathfrak{D a i}$ are respectively interpreted as failure (deadlock) or success of the interaction process. More precisely, either normalization gives no output at all - and in this case the result is the empty strategy, i.e., $\mathfrak{F i d}$ - or it succeeds by reaching the action $\dagger$, which signals termination - and in this case the result is the strategy $\mathfrak{D a i}$. In the case of success, we say that the strategies are orthogonal.

The orthogonality relation is defined only on total strategies. In fact, we already know (Example 4.27) that if $\mathfrak{F i d} \in \mathcal{R}$ then $\llbracket \mathcal{R} \rrbracket=\mathfrak{F i d}$, whatever are the other strategies.

Let us first anticipate the definition in the key case of strategies on a unary interface, and then give the general definition of being orthogonal.

Given two total strategies $\mathcal{D}: \xi^{+}$and $\mathcal{E}: \xi^{-}$, they are said to be orthogonal, written $\mathcal{D} \perp \mathcal{E}$ (or equivalently $\mathcal{E} \perp \mathcal{D}$ ), if $\llbracket \mathcal{D}, \mathcal{E} \rrbracket=\mathfrak{D} \mathfrak{a i}$.

Orthogonality means that at each step of the interaction, the positive action $x^{+}$is matched with its negative dual action $x^{-}$, and the computation terminates by reaching a $\dagger$ action.

We can then define the orthogonal set of a set of total strategies $\mathbf{S}$ on the same interface $\xi^{\epsilon}$ in a standard way as

$$
\mathbf{S}^{\perp}=\left\{\mathcal{E}: \mathcal{E} \text { is a total strategy on the interface } \xi^{\bar{\epsilon}} \text { and } \mathcal{E} \perp \mathcal{D} \text { for any } \mathcal{D} \in \mathbf{S}\right\}
$$

Notice that (as in [22]) the partial strategy $\mathfrak{F} \mathfrak{i d}$ is ruled out in the definition of the orthogonal sets. For instance, if $\mathbf{S}$ is the empty set of negative strategies on $\xi^{-}$, then $\mathbf{S}^{\perp}$ is the set of all total strategies on $\xi^{+}$(in particular, $\mathfrak{F i d} \notin \mathbf{S}^{\perp}$ ).

Example 4.28. In example 4.17, $\mathcal{D} \perp \mathcal{F}$, while $\mathcal{E}$ and $\mathcal{F}$ are not orthogonal.

We now make this notion general.

Definition 4.29 (Counter-strategies). Given an interface $\Gamma$, we call family of counterstrategies (w.r.t. $\Gamma$ ) any family of total strategies $\left(\mathcal{E}_{\xi}: \xi^{\bar{\epsilon}}\right)_{\xi^{\epsilon} \in \Gamma}$.

With a slight abuse of notation, we will write simply $\left(\mathcal{E}_{\xi}\right)_{\xi \in \Gamma}$, by omitting the indication of the polarity (when clear from the context, we will also omit the indication of the indexing set $\Gamma$ ).

If $\mathcal{D}: \Gamma$ is a strategy, we will use the notation $\left\{\mathcal{D},\left(\mathcal{E}_{\xi}\right)\right\}$ for the cut-net $\{\mathcal{D}\} \cup\left\{\mathcal{E}_{\xi}: \xi \in \Gamma\right\}$ they induce. Observe that $\left\{\mathcal{D},\left(\mathcal{E}_{\xi}\right)\right\}$ is a closed cut-net.

Example 4.30. If $\Gamma=\xi^{+}$, a counter-strategy has the form $\mathcal{E}: \xi^{-}$.

If $\Gamma=\xi^{-}, \alpha^{+}, \beta^{+}$, then $\left\{\mathcal{E}_{1}: \xi^{+}, \quad \mathcal{E}_{2}: \alpha^{-}, \quad \mathcal{E}_{3}: \beta^{-}\right\}$is a family of counter-strategies.

We can now define the orthogonality relation and orthogonal sets. We use the same notation of Definition 4.29.

Definition 4.31 (Orthogonality, orthogonal set). Let $\mathcal{D}: \Gamma$ be a total strategy and $\left(\mathcal{E}_{\xi}\right)$ be a family of counter-strategies w.r.t. $\Gamma$. $\mathcal{D}$ and $\left(\mathcal{E}_{\xi}\right)$ are said to be orthogonal, written $\mathcal{D} \perp\left(\mathcal{E}_{\xi}\right)$ (or equivalently $\left.\left(\mathcal{E}_{\xi}\right) \perp \mathcal{D}\right)$, if $\llbracket \mathcal{D},\left(\mathcal{E}_{\xi}\right) \rrbracket$ is total. In other words, we have orthogonality if $\llbracket \mathcal{D},\left(\mathcal{E}_{\xi}\right) \rrbracket=\mathfrak{D a i}$. 
Given a set $\mathbf{S}$ of total strategies on the same interface $\Gamma$, its orthogonal set is defined as

$\mathbf{S}^{\perp}:=\left\{\left(\mathcal{E}_{\xi}\right):\left(\mathcal{E}_{\xi}\right)\right.$ is a family of counter-strategies w.r.t. $\Gamma$ and $\left(\mathcal{E}_{\xi}\right) \perp \mathcal{D}$ for any $\left.\mathcal{D} \in \mathbf{S}\right\}$.

Similarly, given a set $\mathbf{C}$ of families of counter-strategies w.r.t. $\Gamma$, its orthogonal set is defined as

$$
\mathbf{C}^{\perp}:=\left\{\mathcal{D}: \mathcal{D} \text { is total on interface } \Gamma \text { and } \mathcal{D} \perp\left(\mathcal{E}_{\xi}\right) \text { for any }\left(\mathcal{E}_{\xi}\right) \in \mathbf{C}\right\} .
$$

Orthogonality satisfies the usual closure properties: if $\mathbf{E}, \mathbf{F}$ are sets of total strategies on the same interface (resp. sets of families of counter-strategies w.r.t. the same interface), then

- $\mathbf{E} \subseteq \mathbf{F}$ implies $\mathbf{F}^{\perp} \subseteq \mathbf{E}^{\perp}$ (and thus $\mathbf{E}^{\perp \perp} \subseteq \mathbf{F}^{\perp \perp}$ );

- $\mathbf{E} \subseteq \mathbf{E}^{\perp \perp}$

- $\mathbf{E}^{\perp}=\mathbf{E}^{\perp \perp \perp}$.

In terms of games, orthogonality allows the players to agree (or not), without this being guaranteed in advance by the type: $\{\mathcal{D}\}^{\perp}$ is the set of the families of counter-strategies which are consensual (i.e., well interact) with $\mathcal{D}$.

\subsection{Interactive types (behaviours).}

Definition 4.32 (Behaviour). A behaviour (or interactive type) on the interface $\Gamma$ is a set $\mathbf{G}$ of strategies $\mathcal{D}: \Gamma$ such that $\mathbf{G}^{\perp \perp}=\mathbf{G}$ (i.e., it is closed by bi-orthogonal). We say that a behaviour $\mathbf{G}$ is positive or negative according to its interface.

We observe that (by the definition of orthogonal set) an interactive type only contains total strategies.

When is useful to emphasize that $\mathbf{G}$ is a set of strategies on the name $\xi$, we may annotate the name $\xi$ as a subscript: $\mathbf{G}_{\xi}$.

Example $4.33(\mathbf{0}, \boldsymbol{\top})$. Given a name $\xi$, the minimal positive behaviour on $\xi^{+}$is the one generated by the empty set of total strategies on $\xi^{+}$. Its orthogonal set consists of all the negative strategies on interface $\xi^{-}$. Let us call $\mathrm{T}$ this set, which is the maximal negative behaviour on $\xi$. By the definition of orthogonality, a strategy in $T^{\perp}$ must be consensual to all negative strategies on interface $\xi^{-}$. But the only strategy which can do this is $\mathfrak{D a i}$. Hence the closure by bi-orthogonal of the empty set of positive total strategies on $\xi^{+}$is $\{\mathfrak{D a \mathfrak { i }}\}$. Let us call $\mathbf{0}$ this behaviour.

In 22 T and $\mathbf{0}$ are the interpretations of the additive constants $\mathrm{T}$ and $\mathbf{0}$ of linear logic respectively, whence their name.

Remark 4.34. In our setting — but not in [22] - behaviours can be empty. While a positive behaviour is never empty (because it contains at least $\mathfrak{D a i}$ ), a negative behaviour can be empty: if $\mathbf{S}$ is the empty set of strategies on a negative interface, $\mathbf{S}=\mathbf{S}^{\perp \perp}$. This difference with [22] is a limit of our choice of "strict linearity" (Section 4.1.2), and will disappear in the non-linear setting.

This mismatch deserves some discussion. The purpose of this and next section is to give a compact but complete presentation of the construction of ludics and of internal completeness in the linear case. In particular, we want to show (in this setting which is easier to grasp) how full completeness follows from internal completeness, and provide a proof which will then be possible to generalize to a full setting in the second part of 
the paper. For this reason, we will restrict our attention to the multiplicatives. To have additive structure would make the behaviours never empty, but require some more technical definitions, without adding anything substantial to our purposes. The small price is the explicit request for the behaviours to be "non-empty" in the various constructions below.

4.5. Linear types constructors. In this section we consider the behaviours which will interpret logical formulas, more precisely multiplicative formulas.

4.5.1. Constructions on strategies. Let $\mathcal{D}_{1}: \xi 1^{-}, \ldots, \mathcal{D}_{n}: \xi n^{-}$with $n \geq 0$ be negative strategies. We obtain a new positive strategy on the interface $\xi^{+}$, denoted by $\mathcal{D}_{1} \bullet \cdots \bullet \mathcal{D}_{n}$, by adding to the union of the strategies the positive root $\left(\xi, I_{n}\right)^{+}$, i.e.,

$$
\mathcal{D}_{1} \bullet \cdots \bullet \mathcal{D}_{n}:=\left(\xi, I_{n}\right)^{+} \cdot\left\{\mathcal{D}_{1}, \ldots, \mathcal{D}_{n}\right\} .
$$

Recall that as we stipulated in Section 3.3, $I_{n}$ denotes either $\{1, \ldots, n\}$ if $n>0$, or $\emptyset$ if $n=0$.

\subsubsection{Constructions on behaviours.}

Definition 4.35 (Tensor/Par of behaviours). Let $\mathbf{N}_{1} \ldots, \mathbf{N}_{n}$ be non-empty negative behaviours on interface $\xi 1^{-}, \ldots, \xi n^{-}$respectively.

We define the set of strategies:

$$
\mathbf{N}_{1} \bullet \cdots \bullet \mathbf{N}_{n}:=\left\{\mathcal{D}_{1} \bullet \cdots \bullet \mathcal{D}_{n}: \mathcal{D}_{i} \in \mathbf{N}_{i} \text { for any } 1 \leq i \leq n\right\}
$$

and the behaviours

$$
\begin{array}{lll}
\mathbf{N}_{1} \otimes \cdots \otimes \mathbf{N}_{n} & :=\left(\mathbf{N}_{1} \bullet \cdots \bullet \mathbf{N}_{n}\right)^{\perp \perp} & \text { positive behaviour on } \xi^{+} ; \\
\mathbf{N}_{1}^{\perp} \not \supset \cdots \ngtr \mathbf{N}_{n}^{\perp} & :=\left(\mathbf{N}_{1} \bullet \cdots \bullet \mathbf{N}_{n}\right)^{\perp} & \text { negative behaviour on } \xi^{-}
\end{array}
$$

We call multiplicative a behaviour which is inductively generated by these constructions.

Remark 4.36 (0-ary case). In the case that $n=0$, the set $\mathbf{N}_{1} \bullet \cdots \bullet \mathbf{N}_{n}$ consists of a unique strategy $\mathcal{D}=(\xi, \emptyset)^{+}$. We have that $\mathbf{N}_{1}^{\perp} \not 2 \ldots \not 8 \mathbf{N}_{n}^{\perp}$ only contains the strategy $\mathcal{E}=(\xi, \emptyset)^{-} \cdot \mathfrak{D a} \mathfrak{i}$ , since there is no other possibility to be orthogonal to $\mathcal{D}$. Finally, $\mathbf{N}_{1} \otimes \cdots \otimes \mathbf{N}_{n}=\{\mathcal{D}, \mathfrak{D} \mathfrak{a}\}$, again because there are no other possibilities.

We denote by 1 the behaviour $\mathbf{N}_{1} \otimes \cdots \otimes \mathbf{N}_{n}$ and by $\perp$ the behaviour $\mathbf{N}_{1}^{\perp} \not \gamma \ldots \gamma \mathbf{N}_{n}^{\perp}$ in the case $n=0$. Of course, both behaviours are multiplicative.

Proposition 4.37. Let $\mathbf{P}=\mathbf{N}_{1} \otimes \cdots \otimes \mathbf{N}_{n}$ and $\mathbf{N}=\mathbf{N}_{1}^{\perp} \not 8 \ldots \not 8 \mathbf{N}_{n}^{\perp}$.

- $\mathbf{P}, \mathbf{N}$ are non-empty;

- $\mathbf{P}, \mathbf{N}$ contain only non-empty strategies.

In particular, multiplicative behaviours are never empty.

Proof. A positive behaviour is never empty, because it contains at least $\mathfrak{D a i}$. Moreover, $\mathfrak{F i d} \notin \mathbf{P}$, by definition of orthogonality. For negatives behaviours generated by our construction, observe that since $\mathbf{N}_{1}, \ldots, \mathbf{N}_{n}$ are non-empty, $\mathbf{N}_{1} \bullet \cdots \bullet \mathbf{N}_{n}$ is non-empty too and by construction all the strategies have the same root $\left(\xi, I_{n}\right)$. A negative behaviour $\mathbf{N}$ of the form $\left(\mathbf{N}_{1} \bullet \cdots \bullet \mathbf{N}_{n}\right)^{\perp}$ is also never empty, because it contains $\mathcal{E}:=\left(\xi, I_{n}\right)^{-} \cdot \dagger$. The empty strategy cannot belong to $\mathbf{N}$, because it is not orthogonal to any strategy in $\mathbf{N}_{1} \bullet \cdots \bullet \mathbf{N}_{n}$. 
4.6. Sequent of behaviours. As a behaviour on a unary interface corresponds in ludics to a logical formula, the notion of sequent of behaviours corresponds to the notion of sequent.

Definition 4.38 (Sequent of behaviours). Let $\Gamma=\xi_{1}^{\epsilon_{1}}, \ldots, \xi_{n}^{\epsilon_{n}}(n \geq 0)$ be an interface, and let $\boldsymbol{\Gamma}=\mathbf{G}_{\xi_{1}}, \ldots, \mathbf{G}_{\xi_{n}}(n \geq 0)$ behaviours of respective polarities $\epsilon_{1}, \ldots, \epsilon_{n}$.

We define a new behaviour on the same interface $\Gamma$, which we call sequent of behaviours and denote by $\vdash \boldsymbol{\Gamma}$, as follows:

$\vdash \boldsymbol{\Gamma}:=\left\{\mathcal{D}: \mathcal{D}\right.$ is total on interface $\Gamma$ and $\mathcal{D} \perp\left\{\mathcal{E}_{1}, \ldots, \mathcal{E}_{n}\right\}$ for all $\left.\mathcal{E}_{1} \in \mathbf{G}_{\xi_{1}}^{\perp}, \ldots, \mathcal{E}_{n} \in \mathbf{G}_{\xi_{n}}^{\perp}\right\}$.

It is clear that a sequent of behaviours is itself a behaviour, since it is the orthogonal set of the set of families of counter-strategies $\left(\mathcal{E}_{1}, \ldots, \mathcal{E}_{n}\right)$ such that $\mathcal{E}_{1} \in \mathbf{G}_{\xi_{1}}^{\perp}, \ldots, \mathcal{E}_{n} \in \mathbf{G}_{\xi_{n}}^{\perp}$.

Observe that:

- $\vdash \mathbf{P}=\mathbf{P}$ and $\vdash \mathbf{N}=\mathbf{N}$.

- if $\Pi$ is empty, $\vdash \mathbf{\Pi}=\{\mathfrak{D a} \mathfrak{a}\}$.

When moving to full completeness (and thus consider sequents of multiplicative behaviours), we will use also the following property, which is immediate by associativity (Theorem 4.25).

Proposition 4.39. Let $\mathbf{A}, \mathbf{G}_{1}, \ldots \mathbf{G}_{n}(n \geq 0)$ be a sequence of multiplicative behaviours, and $\boldsymbol{\Gamma}=\mathbf{G}_{1}, \ldots \mathbf{G}_{n}$. We have that:

- $\mathcal{D} \in \vdash \boldsymbol{\Gamma}, \mathbf{A}$ if and only if for each $\mathcal{F} \in \mathbf{A}^{\perp}, \llbracket \mathcal{D}, \mathcal{F} \rrbracket \in \vdash \boldsymbol{\Gamma}$.

- $\mathcal{D} \in \vdash \mathbf{\Gamma}, \mathbf{A}$ if and only if $\llbracket \mathcal{D},\left(\mathcal{E}_{i}\right) \rrbracket \in \vdash \mathbf{A}$, for each family $\left(\mathcal{E}_{i}\right)$ such that $\mathcal{E}_{1} \in \mathbf{G}_{1}^{\perp}, \ldots$, $\mathcal{E}_{n} \in \mathbf{G}_{n}^{\perp}$.

Proof. Let us abbreviate the set $\left\{\left(\mathcal{E}_{i}\right): \mathcal{E}_{1} \in \mathbf{G}_{1}^{\perp}, \ldots, \mathcal{E}_{n} \in \mathbf{G}_{n}^{\perp}\right\}$ by $\mathbf{C}$. We then have:

$$
\begin{aligned}
\mathcal{D} \in \vdash \boldsymbol{\Gamma}, \mathbf{A} & \Leftrightarrow \forall \mathcal{F} \in \mathbf{A}^{\perp} \forall\left(\mathcal{E}_{i}\right) \in \mathbf{C}, \mathcal{D} \perp\left\{\mathcal{F},\left(\mathcal{E}_{i}\right)\right\} \\
& \Leftrightarrow \forall \mathcal{F} \in \mathbf{A}^{\perp} \forall\left(\mathcal{E}_{i}\right) \in \mathbf{C}, \llbracket \mathcal{D}, \mathcal{F},\left(\mathcal{E}_{i}\right) \rrbracket \text { is total } \\
& \Leftrightarrow \forall \mathcal{F} \in \mathbf{A}^{\perp} \forall\left(\mathcal{E}_{i}\right) \in \mathbf{C}, \llbracket \llbracket \mathcal{D}, \mathcal{F} \rrbracket,\left(\mathcal{E}_{i}\right) \rrbracket \text { is total (associativity) } \\
& \Leftrightarrow \forall \mathcal{F} \in \mathbf{A}^{\perp} \forall\left(\mathcal{E}_{i}\right) \in \mathbf{C}, \llbracket \mathcal{D}, \mathcal{F} \rrbracket \perp\left(\mathcal{E}_{i}\right) \\
& \Leftrightarrow \forall \mathcal{F} \in \mathbf{A}^{\perp}, \llbracket \mathcal{D}, \mathcal{F} \rrbracket \in \vdash \mathbf{\Gamma} .
\end{aligned}
$$

The second claim is obtained by iterating the first one.

\section{LUDICS IN THE LINEAR CASE: INTERNAL AND FULL COMPLETENESS}

We introduce the notion of internal completeness and give a direct proof of internal completeness, as well as full completeness, without relying on separation.

In [22, the set of strategies which interprets MALL formulas satisfies a remarkable closure property, called internal completeness: the set $\mathbf{S}$ of strategies produced by the construction is essentially equal to its biorthogonal $\left(\mathbf{S}=\mathbf{S}^{\perp \perp}\right)$. Since the biorthogonal does not introduce new objects, we have a complete description of all strategies in the behaviour.

The best example is the interpretation $\mathbf{N}_{1} \otimes \mathbf{N}_{2}:=\left(\mathbf{N}_{1} \bullet \mathbf{N}_{2}\right)^{\perp \perp}$ of a tensor formula. One proves that $\left(\mathbf{N}_{1} \bullet \mathbf{N}_{2}\right) \cup\{\mathfrak{D a i}\}=\left(\mathbf{N}_{1} \bullet \mathbf{N}_{2}\right)^{\perp \perp}$, i.e., we do not add new objects when closing by biorthogonal: our description - the one which generates the set — is already complete. 
From this, full completeness follows. In fact, because of internal completeness, if $\mathcal{D} \in$ $\mathbf{N}_{1} \otimes \mathbf{N}_{2}$ and $\mathcal{D} \neq \mathfrak{D} \mathfrak{a} \mathfrak{i}$ we know we can decompose it as $\mathcal{D}_{1} \bullet \mathcal{D}_{2}$, with $\mathcal{D}_{1} \in \mathbf{N}_{1}$ and $\mathcal{D}_{2} \in \mathbf{N}_{2}$. This corresponds to writing the rule:

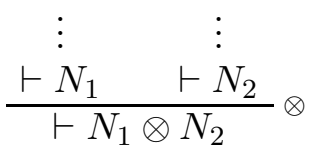

i.e., if each $\mathcal{D}_{i}$ corresponds to a proof of $N_{i}$, and $\mathcal{D}$ corresponds to a proof of $N_{1} \otimes N_{2}$.

5.1. Internal completeness. Until the end of Section 5, we assume the following:

- we fix a name $\xi$

- $\mathbf{N}_{1}, \ldots \mathbf{N}_{n}(n \geq 0)$ are non-empty negative behaviours, respectively on $\xi 1^{-}, \ldots, \xi n^{-}$.

Let us examine the form of the strategies which inhabit a behaviour of the type we have introduced in the last section. Let us first consider $\mathbf{N}_{1} \bullet \mathbf{N}_{2}$. By construction, each strategy in $\mathbf{N}_{1} \bullet \mathbf{N}_{2}$ is on $\xi^{+}$and has $x^{+}=(\xi,\{1,2\})^{+}$as root.

What is $\left(\mathbf{N}_{1} \bullet \mathbf{N}_{2}\right)^{\perp}$ ? By definition of linear normalization, each strategy has as root the action $x^{-}=(\xi,\{1,2\})^{-}$(otherwise, normalization would fail immediately). In particular we have the strategy $x^{-} . \dagger$.

What is $\left(\mathbf{N}_{1} \bullet \mathbf{N}_{2}\right)^{\perp \perp}$ ? All strategies have a positive root, which, to normalize against $\left(\mathbf{N}_{1} \bullet \mathbf{N}_{2}\right)^{\perp}$, must be either $\dagger$, or $x^{+}$. Hence, we know that a strategy $\mathcal{D} \in \mathbf{N}_{1} \otimes \mathbf{N}_{2}$ is either $\mathfrak{D a i}$ or has the form $x^{+} .\left\{\mathcal{D}_{1}, \mathcal{D}_{2}\right\}$, where $\mathcal{D}_{1}: \xi 1$ and $\mathcal{D}_{2}: \xi 2$. The following picture represents this.
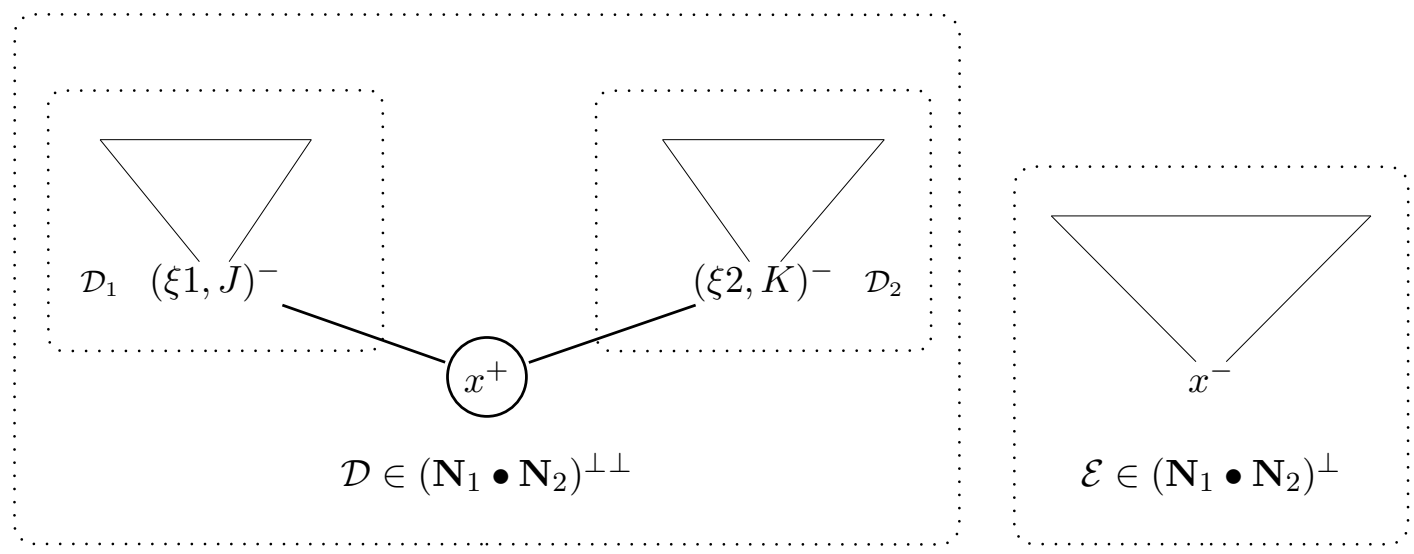

We now want to prove that if $\mathcal{D} \in\left(\mathbf{N}_{1} \otimes \mathbf{N}_{2}\right.$ ) (and $\mathcal{D} \neq \mathfrak{D a i}$ ) then $\mathcal{D}_{1} \in \mathbf{N}_{1}$ and $\mathcal{D}_{2} \in \mathbf{N}_{2}$, which means that $\left(\mathbf{N}_{1} \bullet \mathbf{N}_{2}\right)$ was already complete, i.e., closed by biorthogonal.

Proposition 5.1 (Internal completeness of tensor). Let $\mathbf{N}_{1}, \ldots, \mathbf{N}_{n}$ be $n \geq 0$ non-empty negative behaviours, respectively on $\xi 1^{-}, \ldots, \xi n^{-}$. We have that

$$
\mathbf{N}_{1} \otimes \cdots \otimes \mathbf{N}_{n}=\mathbf{N}_{1} \bullet \cdots \bullet \mathbf{N}_{n} \cup\{\mathfrak{D a i}\} .
$$

Proof. We have already shown that this is true in the case $n=0$ (Remark 4.36). W.l.o.g., we prove the claim in the case $n=2$.

Let $\mathcal{D} \in \mathbf{N}_{1} \otimes \mathbf{N}_{2}, \mathcal{D} \neq \mathfrak{D} \mathfrak{a i}$. We know (by the discussion above) that $\mathcal{D}$ has the form $x^{+} .\left\{\mathcal{D}_{1}, \mathcal{D}_{2}\right\}$, with $x=(\xi,\{1,2\})$. We now prove that $\mathcal{D}_{1} \in \mathbf{N}_{1}$ and $\mathcal{D}_{2} \in \mathbf{N}_{2}$. 
(i) Given any $\mathcal{E}: \xi 1^{+} \in \mathbf{N}_{1}^{\perp}$, we obtain the strategy $\mathcal{E}^{\prime}: \xi^{-}=x^{-} \cdot \mathcal{E}$ by adding the root $x^{-}$. We have that

$$
\llbracket x^{-} \cdot \mathcal{E}, x^{+} .\left\{\mathcal{D}_{1}, \mathcal{D}_{2}\right\} \rrbracket=\llbracket \mathcal{E}, \mathcal{D}_{1} \rrbracket
$$

by definition of normalization, and by the fact that since in $\mathcal{E}$ there are only names generated by $\xi 1$. Hence, $\mathcal{E}: \xi 1^{+}$only interact with the subtree $\mathcal{D}_{1}: \xi 1^{-}$. No action in $\mathcal{D}_{2}$ is ever used.

(ii) $\mathcal{E}^{\prime} \in\left(\mathbf{N}_{1} \bullet \mathbf{N}_{2}\right)^{\perp}$, because by using (因) we deduce that $\mathcal{E}^{\prime} \perp \mathcal{D}$, for any $\mathcal{D} \in\left(\mathbf{N}_{1} \bullet \mathbf{N}_{2}\right)$.

(iii) Given any $\mathcal{D} \in \mathbf{N}_{1} \otimes \mathbf{N}_{2}$, by definition we have that $\mathcal{D} \perp \mathcal{E}$ for each $\mathcal{E} \in\left(\mathbf{N}_{1} \bullet \mathbf{N}_{2}\right)^{\perp}$. Hence in particular, for each $\mathcal{E} \in \mathbf{N}_{1}^{\perp}$, we have $\mathcal{D} \perp \mathcal{E}^{\prime}\left(\mathcal{E}^{\prime}\right.$ defined as above). Again because of (因), $\mathcal{D}_{1} \perp \mathcal{E}$. This says that $\mathcal{D}_{1} \in\left(\mathbf{N}_{1}^{\perp}\right)^{\perp}=\mathbf{N}_{1}$.

Remark 5.2 (Important). The key to extend this argument to the case of ludics with repetition, is that here we only use two properties of the strategies: the dynamics (normalization), and the fact that the root is the only action on the name $\xi$ (to say at point (i) in the proof above that occurrences of $\xi 1$ only appear inside $\mathcal{D}_{1}$ ).

Proposition 5.3 (Internal completeness of par). Let $\mathbf{N}_{1}, \ldots, \mathbf{N}_{n}$ be as in 5.1 (hence $\mathbf{N}_{1}^{\perp}, \ldots, \mathbf{N}_{n}^{\perp}$ are positive behaviours respectively on $\left.\xi 1^{+}, \ldots, \xi n^{+}\right)$, and let $x=\left(\xi, I_{n}\right)$. We have:

$$
x^{-} . \mathcal{E} \in \mathbf{N}_{1}^{\perp} \not \supset \ldots \not \gamma \mathbf{N}_{n}^{\perp} \Leftrightarrow \mathcal{E} \in \vdash \mathbf{N}_{1}^{\perp}, \ldots, \mathbf{N}_{n}^{\perp} .
$$

Proof. The case $n=0$ is actually given by Remark 4.36, observing that the empty sequent of behaviours contains only $\mathfrak{D a i}$.

Let us give the proof in the case $n=2$. A strategy $x^{-}$. $\mathcal{E}$ belongs to $\mathbf{N}_{1}^{\perp} \not \gamma \mathbf{N}_{2}^{\perp}$ if and only if for any $x^{+} .\left\{\mathcal{D}_{1}, \mathcal{D}_{2}\right\} \in \mathbf{N}_{1} \bullet \mathbf{N}_{2}$, we have that $x^{-} \cdot \mathcal{E} \perp x^{+} .\left\{\mathcal{D}_{1}, \mathcal{D}_{2}\right\}$. By definition of normalization, $\llbracket x^{-} \cdot \mathcal{E}, x^{+} .\left\{\mathcal{D}_{1}, \mathcal{D}_{2}\right\} \rrbracket=\llbracket \mathcal{E}, \mathcal{D}_{1}, \mathcal{D}_{2} \rrbracket$, and from this and the definition of sequent of behaviours (Definition 4.38) the claim follows immediately.

5.2. Full completeness. We are now ready to show how full completeness is obtained from internal completeness, in the linear case. More precisely, we are going to introduce the calculus MLLS, which is the multiplicative fragment of the calculus HS introduced by Girard in [22, 23. We then show that full completeness can be derived from internal completeness of tensor and par and Proposition 4.39.

While the proof in 22] relies on separation, we give a direct proof which uses only the properties of the dynamics. The choice of omitting the additive here only simplifies (and shortens) the presentation; the proof can be extended to the additive structure without problems (but in that case, we would need to add one more "winning conditions", as we will discuss below).

5.2.1. MLLS. As it is the case for HS 22, 23], the calculus MLLS is affine, i.e., we have weakening (but restricted to positive formulas).

The calculus MLLS can be seen as the affine (with implicit weakening but without implicit contraction for positive formulas) restriction of the calculus MELLS given in Section $23^{3}$.

Formulas of MLLS are inductively given by:

$$
P::=N_{1} \otimes \cdots \otimes N_{n} \quad N::=P_{1} \not 8 \ldots \not 8 P_{n} \quad(n \geq 0) .
$$

\footnotetext{
${ }^{3}$ We do not consider the cut-rule here, but we do it in Section 11.3
} 
When $n=0$, we write I and $\perp$ for the positive and negative formula respectively.

Rules of MLLS are given in Table 3 .

$$
\begin{aligned}
& \text { Positive rules : } \quad \frac{\vdash \Pi_{1}, N_{1} \ldots \quad \vdash \Pi_{n}, N_{n}}{\vdash \Pi_{0}, \Pi_{1}, \ldots, \Pi_{n}, P} \operatorname{Pos}_{n} \\
& P=N_{1} \otimes \cdots \otimes N_{n} \text { and } n \geq 0 \\
& \text { Negative rules : } \quad \frac{\vdash \Pi, P_{1}, \ldots, P_{n}}{\vdash \Pi, N} \mathrm{Neg}_{n} \\
& N=P_{1} \not 2 \ldots>P_{n} \text { and } n \geq 0
\end{aligned}
$$

Table 3: MLLS

Notice the (implicit) weakening on occurrences of positive formulas $\Pi_{0}$ in the positive rule.

5.2.2. Interpretation. We only give an outline of the interpretation of formulas and sequents. We will discuss it in full detail the interpretation of derivations in the setting which also includes exponentials in Section 11.3.

Given a formula $F$ of MLLS and an arbitrary name $\xi$ we associate a multiplicative behaviour of the same polarity $\mathbf{F}$ on interface $\xi$ inductively as follows.

$$
\begin{gathered}
N_{1} \otimes \cdots \otimes N_{n} \xi:=N_{1} \xi 1 \otimes \cdots \otimes N_{n} \xi n \\
P_{1} \ngtr \ldots>P_{n} \xi:=P_{1} \xi 1 \not 2 \ldots 8 P_{n} \xi n .
\end{gathered}
$$

Given a positive sequent $\vdash P_{1}, \ldots, P_{n}$, and a positive interface $\xi_{1}^{+} \ldots, \xi_{n}^{+}$we associate the sequent of behaviours $\vdash P_{1} \xi_{1}, \ldots, P_{n} \xi_{n}$. Given a negative sequent $\vdash N, P_{1}, \ldots, P_{n}$ and a negative interface $\sigma^{-}, \xi_{1}^{+} \ldots, \xi_{n}^{+}$we associate the sequent of behaviours $\vdash N^{\sigma}, P_{1} \xi_{1}, \ldots, P_{n} \xi_{n}$.

The interpretation of a proof $\pi$ of $\vdash \Gamma$ will be a daimon-free strategy in the sequent of behaviours $\vdash \boldsymbol{\Gamma}$. Once given the interpretation of proofs (which we only do in Section 11.5) one can establish the following theorem.

Theorem 5.4. Let $\pi$ be a proof of a sequent $\vdash \Gamma$ in MLLS. There exists a daimon-free strategy $\mathcal{D} \in \vdash \boldsymbol{\Gamma}$ such that $\mathcal{D}$ is interpretation of $\pi$.

5.2.3. Full completeness. We now show the following in detail:

Theorem 5.5 (Full completeness). Let $\vdash \boldsymbol{\Gamma}$ be a sequent of behaviours which is interpretation of the sequent $\vdash \Gamma$ in MLLS. If $\mathcal{D}$ is a daimon-free strategy in $\vdash \boldsymbol{\Gamma}$ then $\mathcal{D}$ is the interpretation of a proof $\pi$ of the sequent $\vdash \Gamma$ in MLLS. 
We notice that we associate proofs only to daimon-free strategies. Being daimon-free is what is called a "winning condition." Since we are only concerned with the multiplicative structure, this is the only condition necessary for linear strategies.

When working with additive structure, or with exponentials, one also needs the notion of materiality, which is introduced in [22] and that we will discuss in Section [11. However, since we restrict out attention to the multiplicative fragment, we can overlook materiality for the moment.

Let $\vdash \boldsymbol{\Gamma}$ be the interpretation of the sequent $\vdash \Gamma$, and $\mathcal{D} \in \vdash \boldsymbol{\Gamma}$ a daimon-free strategy. Our purpose is to associate to $\mathcal{D}$ a derivation $\mathcal{D}^{\star}$ of $\vdash \Gamma$ in MLLS by progressively decomposing $\mathcal{D}$, i.e., inductively writing "the last rule." To be able to use internal completeness, which is defined on behaviours on unary interfaces (and not on sequents of behaviours), we will use - back and forth - the definition of sequent of behaviours and in particular Proposition 4.39,

The formula on which the last rule is applied is indicated by the name of the root action. For example, let us assume that the root of $\mathcal{D}$ is $(\xi, I)$; then if $\mathcal{D} \in \vdash \mathbf{G}_{\alpha}, \mathbf{F}_{\xi}$, the behaviour which corresponds to the last rule is the one on $\xi$, that is $\mathbf{F}_{\xi}$.

The proof is by induction on the number of logical symbols occurring in the sequent $\vdash \Gamma$ we have interpreted in $\vdash \boldsymbol{\Gamma}$.

In the sequel, we consider sequents of behaviours of the form $\vdash \mathbf{\Pi}, \mathbf{F}_{\xi}$, which are interpretations of sequents $\vdash \Pi, F$ of MLLS. $\mathbf{F}$ is the interpretation of a formula $F$ and $\Pi$ is a sequence of $m$ behaviours $\mathbf{Q}_{\alpha_{1}}, \ldots, \mathbf{Q}_{\alpha_{m}}$ which respectively interpret formulas $Q_{1}, \ldots, Q_{m}$. Observe that by the shape of the rules of MLLS and the fact that the interpretation of formulas preserves the polarity, $\boldsymbol{\Pi}$ always consists of positive behaviours only.

We also remark the following facts:

- Since we consider daimon-free strategies, all the actions in $\mathcal{D}$ are proper actions.

- By linearity, both positive and negative strategies have a single root (i.e., they are trees, and not forests), and hence are of the form $\mathcal{D}=x^{+} .\left\{\mathcal{D}_{1}, \ldots, \mathcal{D}_{n}\right\}$ or $\mathcal{D}=x^{-} . \mathcal{D}^{\prime}$.

- Suppose that $\mathcal{D}$ belongs to some negative behaviour $\mathbf{F}$ which is interpretation of a formula of MLLS. Since $\mathbf{F}$ has to be of the form $\left(\mathbf{N}_{1} \bullet \cdots \bullet \mathbf{N}_{n}\right)^{\perp}$, then $\mathcal{D}$ is not empty (see Proposition 4.37).

Below we use the following convention: we write $(\mathcal{E})$ and $(\mathcal{E}) \in \mathbf{C}$ for $\mathcal{E}_{1}, \ldots, \mathcal{E}_{m}$ and $\mathcal{E}_{1} \in \mathbf{Q}_{\alpha_{1}}^{\perp}, \ldots, \mathcal{E}_{m} \in \mathbf{Q}_{\alpha_{m}}^{\perp}$ respectively.

We have two cases.

Positive case. Let $\mathcal{D}=\left(\xi, I_{n}\right)^{+} .\left\{\mathcal{D}_{1}, \ldots, \mathcal{D}_{n}\right\}$ be a positive daimon-free strategy which belongs to $\vdash \boldsymbol{\Pi}, \mathbf{F}_{\xi}$. Let $\mathbf{F}_{\xi}=\mathbf{N}_{1} \otimes \cdots \otimes \mathbf{N}_{k}$. By Proposition 4.39, for any $(\mathcal{E}) \in \mathbf{C}$, we have that $\llbracket \mathcal{D},(\mathcal{E}) \rrbracket \in \mathbf{F}_{\xi}$, hence its root is $\left(\xi, I_{k}\right)$. By definition of normalization, the root of $\llbracket \mathcal{D},(\mathcal{E}) \rrbracket$ is still $\left(\xi, I_{n}\right)$ (because it is a visible action), hence $k=n$.

We proceed as follows, using internal completeness.

(1) Let $\mathcal{C}=\llbracket \mathcal{D},(\mathcal{E}) \rrbracket$. By internal completeness of the tensor (Proposition [5.1), we have that $\mathcal{C}$ can be written as $\mathcal{C}_{1} \bullet \cdots \bullet \mathcal{C}_{n}$, for some $\mathcal{C}_{i} \in \vdash \mathbf{N}_{i}$.

(2) By linearity, observe that a name $\alpha \in\left\{\alpha_{1}, \ldots, \alpha_{m}\right\}$ of the context $\mathbf{\Pi}=\mathbf{Q}_{\alpha_{1}}, \ldots, \mathbf{Q}_{\alpha_{m}}$ either appears in one of the $\mathcal{D}_{i}$ - and there is only one such a $\mathcal{D}_{i}$ - or it does not appear at all. This allows us to univocally split the context $\boldsymbol{\Pi}$ into disjoint subsets as follows. We define:

a. $\boldsymbol{\Pi}_{i}:=\left\{\mathbf{Q}_{\alpha} \in \boldsymbol{\Pi}\right.$ : the name $\alpha$ occurs in $\left.\mathcal{D}_{i}\right\}$, for $1 \leq i \leq n$; 
b. $\Pi_{0}:=\Pi \backslash \bigcup_{1 \leq i \leq n} \Pi_{i}$.

Let us split $(\mathcal{E}) \in \mathbf{C}$ into $(\mathcal{E})_{0} \in \mathbf{C}_{0},(\mathcal{E})_{1} \in \mathbf{C}_{1} \ldots,(\mathcal{E})_{n} \in \mathbf{C}_{n}$. By definition of normalization we have:

$$
\begin{aligned}
\mathcal{C}=\llbracket x^{+} .\left\{\mathcal{D}_{1}, \ldots, \mathcal{D}_{n}\right\},(\mathcal{E}) \rrbracket & =x^{+} .\left\{\llbracket \mathcal{D}_{1},(\mathcal{E})_{1} \rrbracket, \ldots, \llbracket \mathcal{D}_{n},(\mathcal{E})_{n} \rrbracket\right\} \\
& =\llbracket \mathcal{D}_{1},(\mathcal{E})_{1} \rrbracket \bullet \cdots \bullet \llbracket \mathcal{D}_{n},(\mathcal{E})_{n} \rrbracket \\
& =\mathcal{C}_{1} \bullet \cdots \bullet \mathcal{C}_{n} .
\end{aligned}
$$

From this, we conclude that $\llbracket \mathcal{D}_{1},(\mathcal{E})_{1} \rrbracket \in \vdash \mathbf{N}_{1}, \ldots, \llbracket \mathcal{D}_{n},(\mathcal{E})_{n} \rrbracket \in \vdash \mathbf{N}_{n}$. By applying Proposition 4.39 again, we have that $\mathcal{D}_{1} \in \vdash \mathbf{\Pi}_{1}, \mathbf{N}_{1}, \ldots, \mathcal{D}_{n} \in \vdash \mathbf{\Pi}_{n}, \mathbf{N}_{n}$. The size has decreased for all sequents, and $\mathcal{D}_{1}, \ldots, \mathcal{D}_{n}$ are obviously all daimon-free. We can then apply the inductive hypothesis and write the derivation:

$$
\begin{array}{cc}
\vdots \mathcal{D}_{1}^{\star} & \vdots \mathcal{D}_{n}^{\star} \\
\vdash \Pi_{1}, N_{1} \quad \ldots \quad \vdash \Pi_{n}, N_{n} \\
\hline \vdash \Pi_{0}, \Pi_{1} \ldots, \Pi_{n}, N_{1} \otimes \cdots \otimes N_{n}
\end{array} \operatorname{Pos}_{n}
$$

Notice that in case $\mathbf{F}_{\xi}=\mathbf{1}$ then $\mathcal{D}=(\xi, \emptyset)^{+}$and the procedure above gives the rule

$$
\overline{\vdash \Pi_{0}, I} \operatorname{Pos}_{0}
$$

Negative case. The negative case is an immediate application of the internal completeness for par (Proposition 5.3) and Proposition 4.39.

Let $\mathcal{D}=x^{-} \cdot \mathcal{D}^{\prime}$, and $x=\left(\xi, I_{n}\right)$. Assume $\mathcal{D} \in \vdash \boldsymbol{\Pi}, \mathbf{F}_{\xi}$ and $\mathcal{D}$ daimon-free, where $\mathbf{F}_{\xi}=\mathbf{P}_{1} \not \gamma \ldots \gamma \mathbf{P}_{n}$ is the interpretation of the formula $P_{1} \not 8 \ldots \gamma P_{n}$ and $\boldsymbol{\Pi}$ is the interpretation of the context $\Pi$ of a negative sequent $\vdash \Pi, P_{1} \not 8 \ldots \gamma P_{n}$ of MLLS.

For any family $(\mathcal{E}) \in \mathbf{C}$, we have

(1) $\llbracket \mathcal{D},(\mathcal{E}) \rrbracket \in \mathbf{P}_{1} \not \supset \ldots \gamma \mathbf{P}_{n}$, and the root is still $x^{-}$. This allows us to use internal completeness.

(2) By internal completeness (Proposition [5.3), we conclude that $\llbracket \mathcal{D},(\mathcal{E}) \rrbracket$ is of the form $x^{-} . \mathcal{D}^{\prime \prime}$ with $\mathcal{D}^{\prime \prime} \in \vdash \mathbf{P}_{1}, \ldots, \mathbf{P}_{n}$.

(3) By the definition of normalization,

$$
\llbracket \mathcal{D},(\mathcal{E}) \rrbracket=\llbracket x^{-} \cdot \mathcal{D}^{\prime},(\mathcal{E}) \rrbracket=x^{-} \cdot \llbracket \mathcal{D}^{\prime},(\mathcal{E}) \rrbracket .
$$

From this, we have that $\mathcal{D}^{\prime \prime}=\llbracket \mathcal{D}^{\prime},(\mathcal{E}) \rrbracket$ and hence $\llbracket \mathcal{D}^{\prime},(\mathcal{E}) \rrbracket \in \vdash \mathbf{P}_{1}, \ldots, \mathbf{P}_{n}$.

By applying Proposition 4.39 again, we have that $\mathcal{D}^{\prime} \in \vdash \boldsymbol{\Pi}, \mathbf{P}_{\xi 1}, \ldots, \mathbf{P}_{\xi n}$. By applying the inductive hypothesis, we can write the derivation:

$$
\begin{gathered}
\vdots \mathcal{D}^{\prime \star} \\
\frac{\vdash \Pi, P_{1}, \ldots, P_{n}}{\vdash \Pi, P_{1} \not \supset \times P_{n}} \mathrm{Neg}_{n}
\end{gathered}
$$




\section{Ludics With REPETITIONS: WHAT, HOW, WHY}

In the previous section, we assumed linearity of the strategies to prove internal completeness. From now on, we go back to the general definition of strategy (on an universal arena) as in the beginning of Section 4, without any hypothesis of linearity. This means that strategies now allow repeated actions.

In this section, we mainly discuss the difficulties in extending the approach of ludics to this setting, and introduce our solution, which will be technically developed in Section 7.

First, let us introduce some operations which we will use in this section to deal with repeated actions and describe the composition.

\subsection{Copies and renaming.}

Renaming. Given a strategy $\mathcal{D}: \xi$ of arbitrary polarity, let us indicate by $\mathcal{D}[\sigma / \xi]$ the strategy obtained from $\mathcal{D}$ by renaming, in all occurrences of action, the prefix $\xi$ into $\sigma$, i.e., each name $\xi . \alpha$ becomes $\sigma . \alpha$. Obviously, if $\mathcal{D}: \xi$, then $\mathcal{D}[\sigma / \xi]: \sigma$.

Renaming of the root. Given a positive strategy $\mathcal{D}: \xi^{+}$, let us indicate by $\underline{\sigma}(\mathcal{D})$ the strategy obtained by renaming the prefix $\xi$ into $\sigma$ in the root, and in all actions which are hereditarily justified by the root. If $\mathcal{D}: \xi^{+}$, we obtain a new strategy $\underline{\sigma}(\mathcal{D}): \sigma^{+}, \xi^{+}$.

We picture both the operations in Figures 2(a) and (b). For readability, we indicate an action on $\xi$ simply with the name $\xi$.

Copies of a behaviour. We remind that to emphasize that $\mathbf{A}$ is a set of strategies on interface $\xi$, we annotate the name $\xi$ as a subscript: $\mathbf{A}_{\xi}$. If $\mathbf{A}_{\xi}$ is a set of strategies on the name $\xi$, we write $\mathbf{A}_{\sigma}$ for $\left\{\mathcal{D}[\sigma / \xi]: \mathcal{D} \in \mathbf{A}_{\xi}\right\}$. $\mathbf{A}_{\sigma}$ is a copy of $\mathbf{A}_{\xi}$ : they are equal up to renaming.

6.2. Composition (normalization). In a strategy, actions can now be repeated. Composition of strategies as sets of views can be described via the VAM abstract machines introduced in [11. We describe composition in details in Section 8 ,

We now give an example of composition of strategies using the graphical notation introduce before.

However, what we will really need is only that composition has a fundamental property, expressed by the following equation:

$$
\llbracket \mathcal{D}, \mathcal{E} \rrbracket=\llbracket \underline{\sigma}(\mathcal{D}), \mathcal{E}, \mathcal{E}[\sigma / \xi] \rrbracket
$$

for any $\mathcal{D}: \xi^{+}$and $\mathcal{E}: \xi^{-}$such that the operations " $\underline{\sigma}(-)$ " and " $[\sigma / \xi]$ " make sense. This property will also hold for strategies with silent actions we introduce later. The proof for a more general equation is given in Section 8 (Proposition 8.15).

Remark 6.1. Equation 6.1 is reminiscent of the separation of head occurrence property of [2] and the linearization of the head occurrence property of [1] (see also the discussion therein).

From Equation (6.1), we have in particular:

Corollary 6.2. $\mathcal{D} \perp \mathcal{E}$ if and only if $\underline{\sigma}(\mathcal{D}) \perp\{\mathcal{E}, \mathcal{E}[\sigma / \xi]\}$. 
(a)
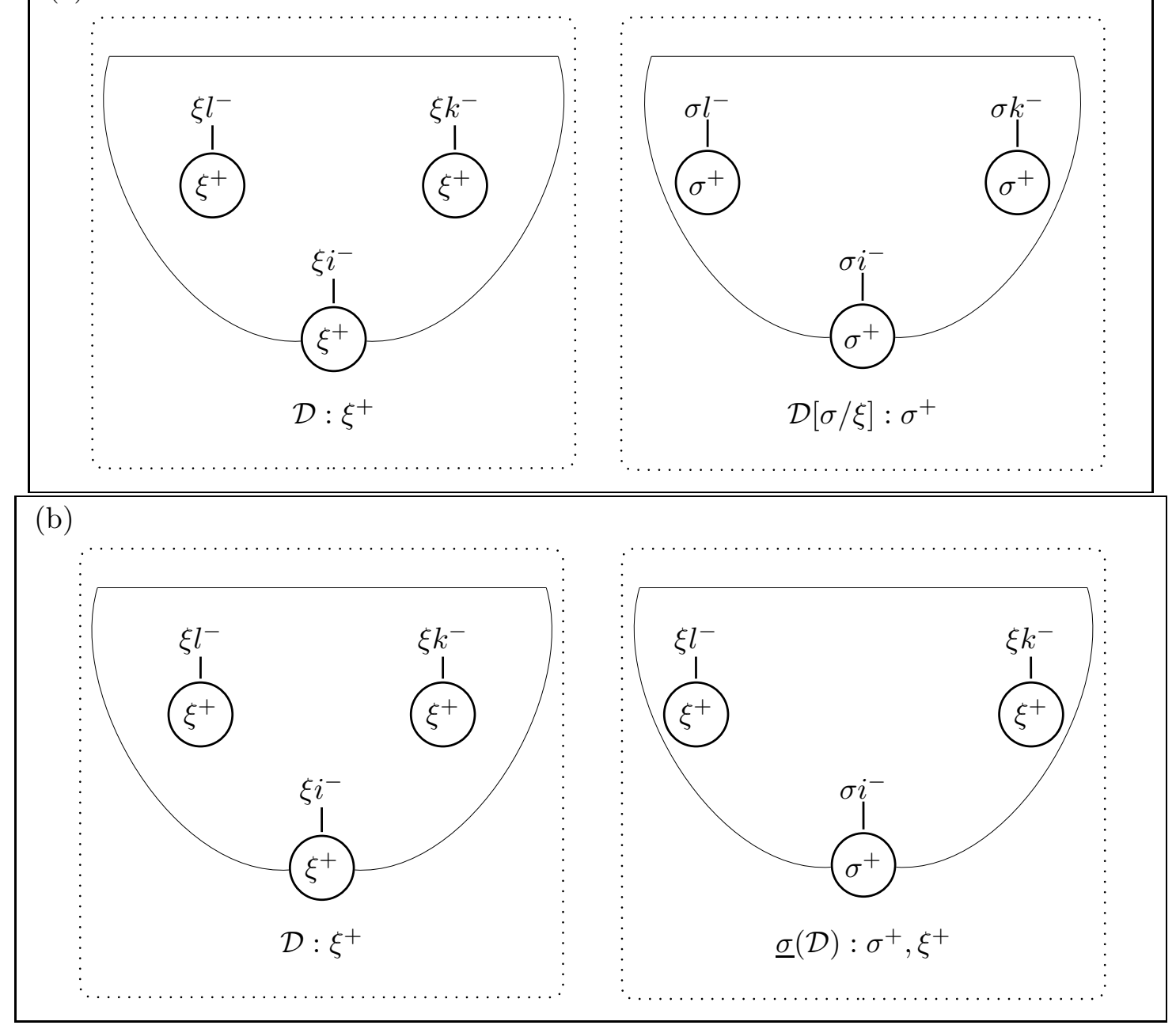

Figure 2: Renaming (a) and renaming of the root (b)

Let us see how Equation (6.1) works by giving a description of the composition.

Let $\mathcal{D}: \xi^{+}$and $\mathcal{E}: \xi^{-}$be two strategies, which we represent in Figure 3 (a) (again, we indicate an action $x$ on $\xi$ simply with the name $\xi$ ). The idea behind the abstract machine in [11] is that, when the two strategies $\mathcal{D}$ and $\mathcal{E}$ interact, every time $\mathcal{D}$ plays an action $x$ on $\xi$, a copy of $\mathcal{E}$ is created; i.e., composition works as if we had a copy of $\mathcal{E}$ for each occurrence of $x$ in $\mathcal{D}$. It is rather intuitive that the result of normalization is the same if we make this explicit, by renaming one occurrence of $x$ (namely the root), and making an explicit copy of $\mathcal{E}$, as illustrated in Figure 3 (b).

Example 6.3. Let us consider the strategies $\mathcal{D}$ and $\mathcal{E}$ in Figure 4 , where we indicate an action $x$ on $\xi$ simply with the name $\xi$. Observe that we explicitly need to draw a pointer from $\xi 11^{+}$to the right occurrence of $\xi 1^{-}$(the lowermost one in our case) which justifies it. The other pointers can be univocally determined. The interaction is the sequence given by following the arrows and the normal form is $\mathfrak{D a i}$. 


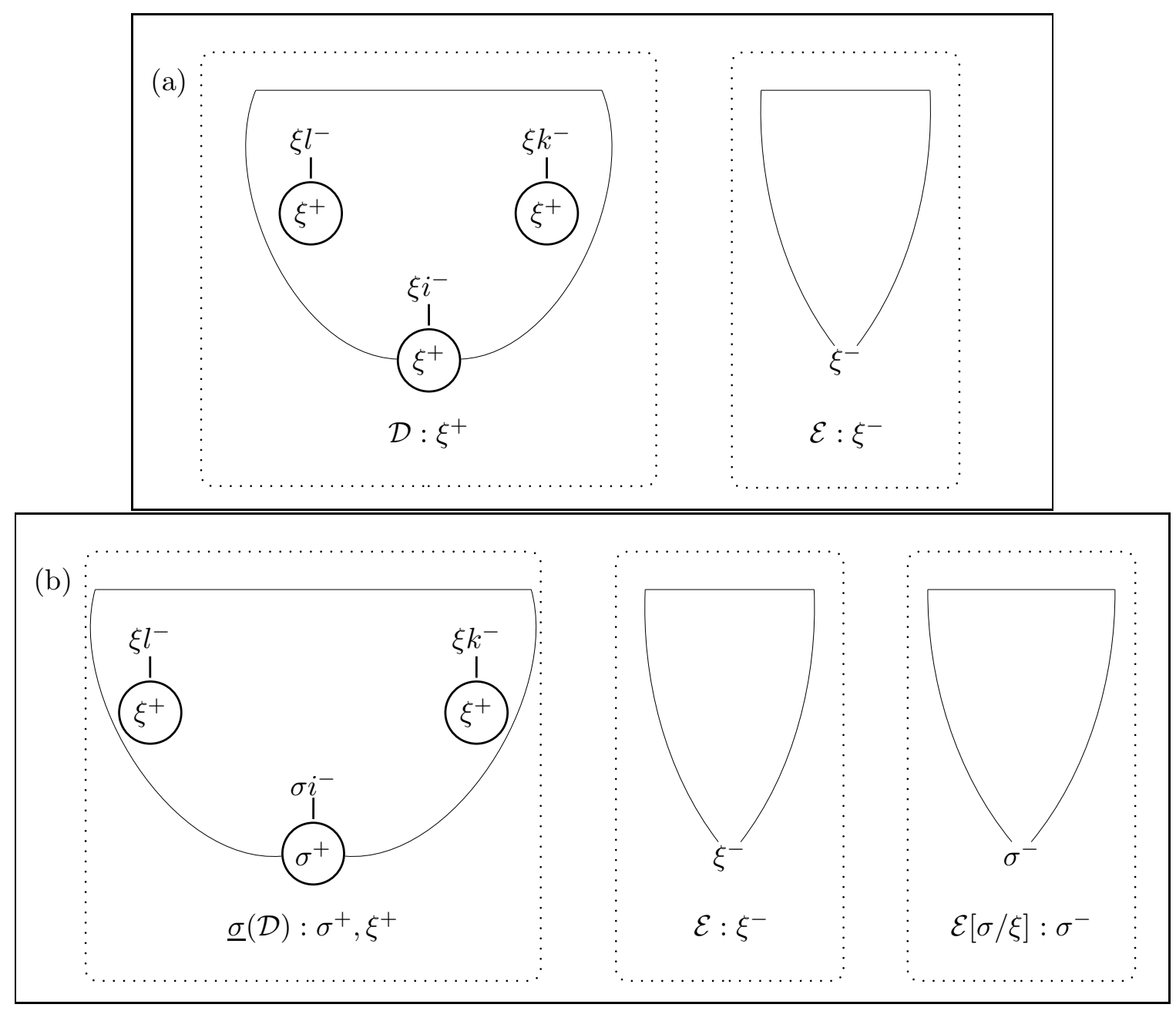

Figure 3: Composition (with repeated actions)

Example 6.4. We now check for $\mathcal{D}, \mathcal{E}$ in Example 6.3 that $\llbracket \mathcal{D}, \mathcal{E} \rrbracket=\llbracket \underline{\sigma}(\mathcal{D}), \mathcal{E}, \mathcal{E}[\sigma / \xi] \rrbracket$ as pictured in Figure 5. Since $\underline{\sigma}(\mathcal{D})$ is linear in this example, we no longer need to show the pointers explicitly.

6.3. What are the difficulties. We are ready to discuss which are the difficulties in extending the approach of ludics to a setting where strategies are non-linear.

Problem 1: Separation. The first problem when strategies have repetitions is with separation. Let us give a simple example of why separation fails if we allow repetitions.

Example 6.5 ([32]). Let $\mathcal{D}_{1}, \mathcal{D}_{2}: \xi^{+}$and $\mathcal{E}: \xi^{-}$be strategies as in Figure 6, where $x=(\xi, I), y=(\xi i, J)$. We cannot find a strategy orthogonal to $\mathcal{D}_{1}$ but not orthogonal to $\mathcal{D}_{2}$. For example, the interactions between $\mathcal{D}_{1}$ and $\mathcal{E}$ and $\mathcal{D}_{2}$ and $\mathcal{E}$ produce the same normal form $\mathfrak{D a i}$. 


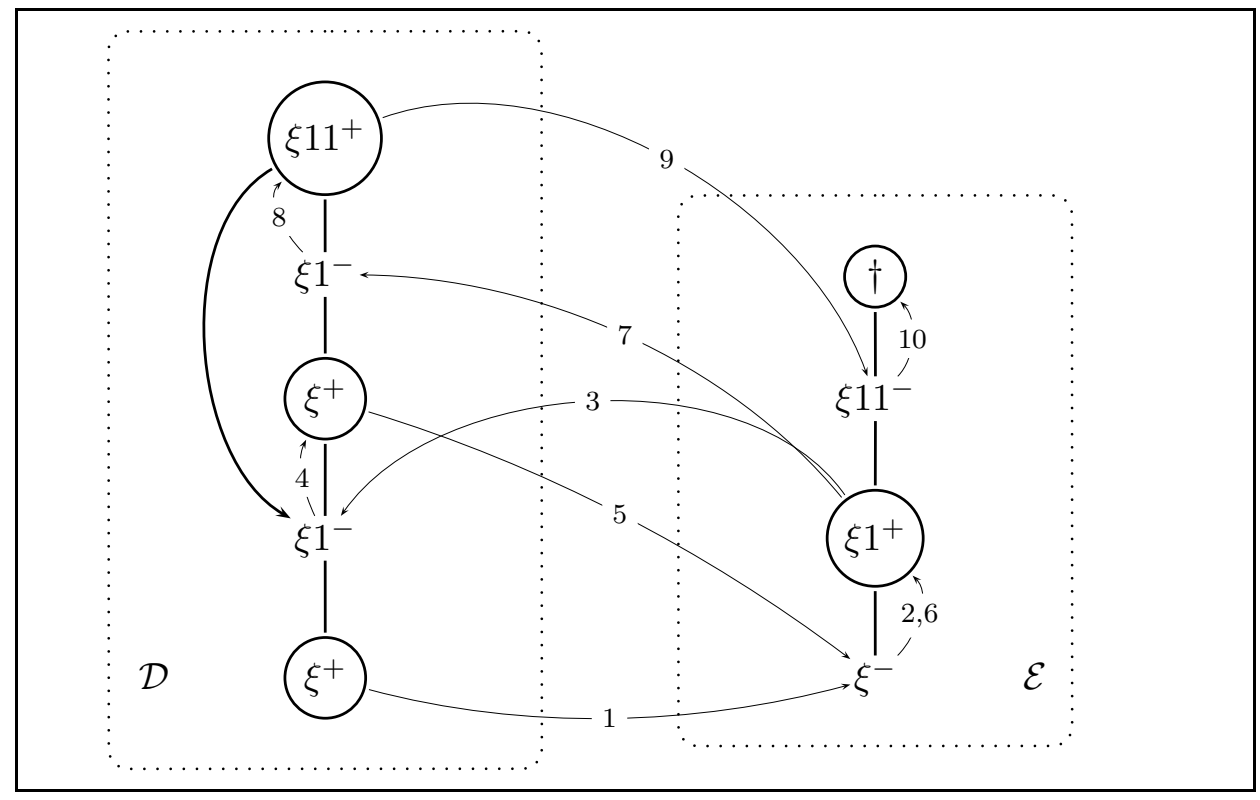

Figure 4: Example of interaction with repetitions

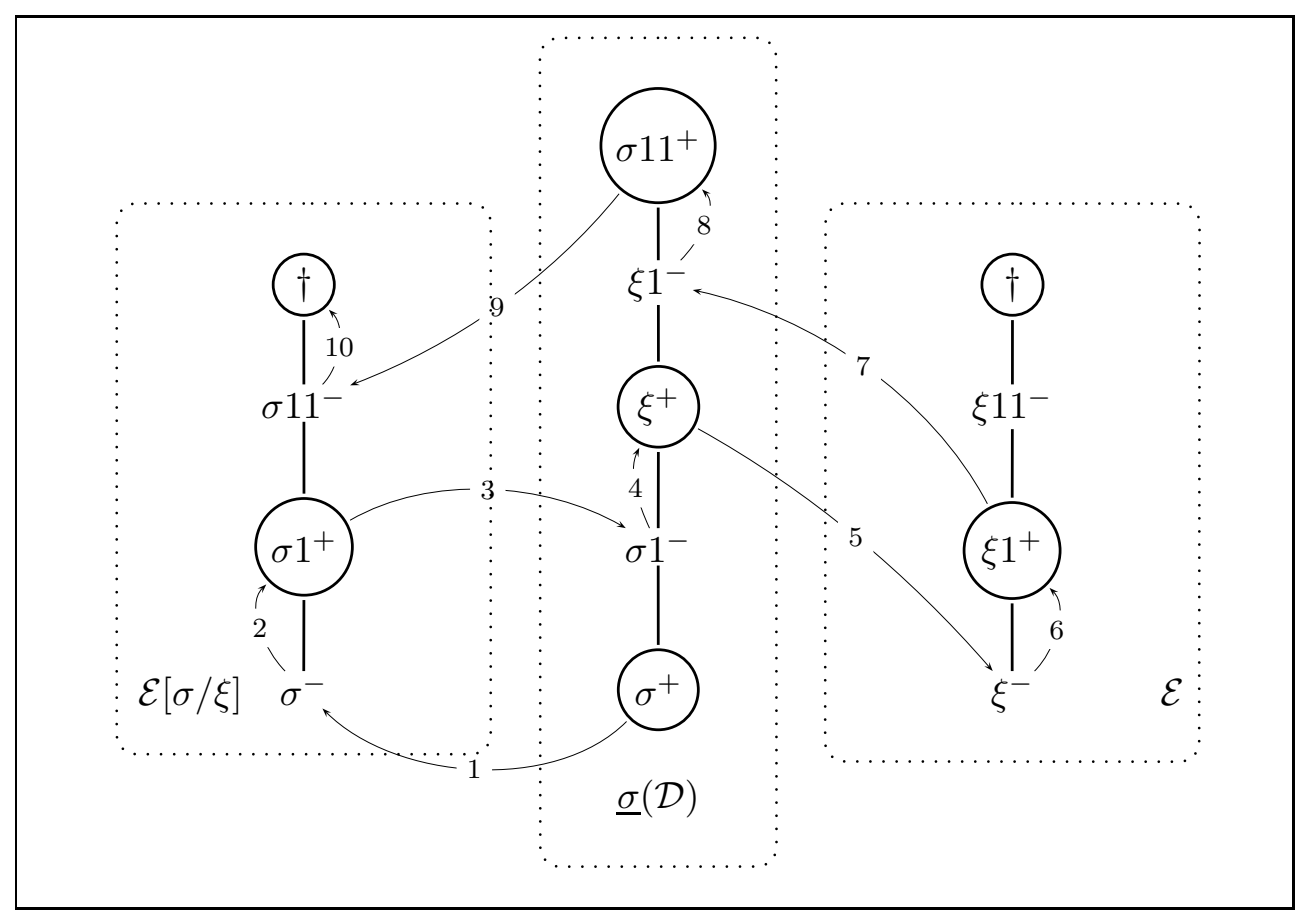

Figure 5: Example of interaction with copies

In this work, we ignore separation all together. As we discussed in Section 5, even if separation is an important property, we don't need it in order to have interactive types and internal completeness. In future work, it may be possible to refine our setting using Maurel's techniques [32]. 


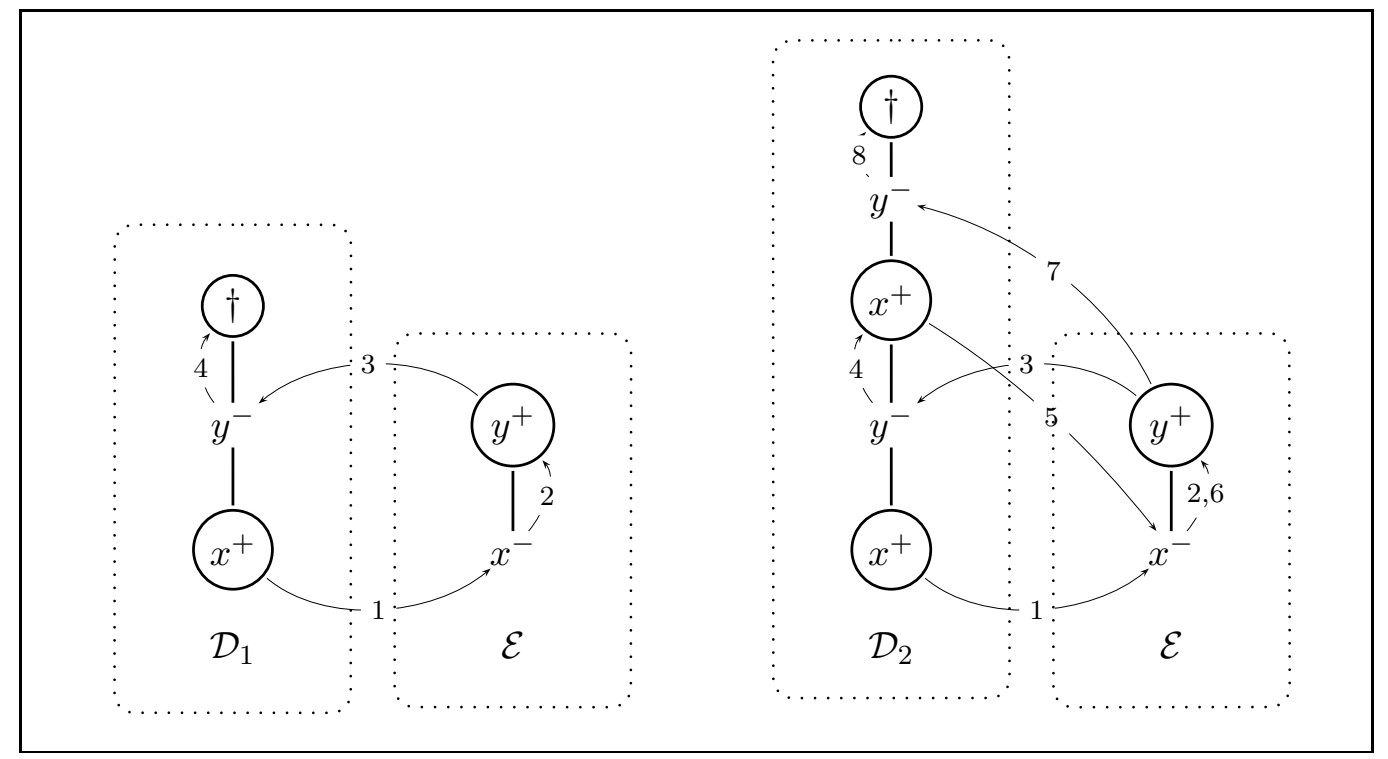

Figure 6: Non-separation

Problem 2: Enough tests (counter-strategies). The second problem - which we believe being the deeper one - has to do with having enough tests, i.e., enough counter-strategies. As in [22], we have defined an interactive type to be any set of strategies closed by biorthogonal. Assume we have defined how to interpret formulas of MELLS, like $P=$ $?_{P}\left(N_{1} \otimes \cdots \otimes N_{n}\right)$ and $P^{\perp}=!_{N}\left(N_{1}^{\perp} \not 2 \ldots \gamma N_{n}^{\perp}\right)$.

We would like to associate to each "good" strategy in the interpretation of a formula, say the positive formula $P$ above, in a behaviour that we indicate with $\mathbf{P}$, a syntactical proof of $\vdash P$ (full completeness). If $\mathcal{D}: \xi^{+} \in \mathbf{P}$, we would like to transform it into a strategy $\mathcal{D}^{\prime} \in \vdash \mathbf{P}_{\xi}, \mathbf{P}_{\sigma}$ (where distinct names indicate distinct copies). This corresponds to the contraction rule (in its upwards reading).

The natural idea is to use the same technique as in [2], and to rename the root, and all the actions which are hereditarily justified by it. We have already illustrated this operation in Section 6.1 (Figure 21). From $\mathcal{D}: \xi^{+}$, we obtain a new strategy $\mathcal{D}^{\prime}: \xi^{+}, \sigma^{+}$, where $\mathcal{D}^{\prime}=\underline{\sigma}(\mathcal{D})$.

We would like to prove that:

$$
(*) \mathcal{D} \in \vdash \mathbf{P}_{\xi} \Rightarrow(* *) \underline{\sigma}(\mathcal{D}) \in \vdash \mathbf{P}_{\xi}, \mathbf{P}_{\sigma} .
$$

To have (**), we need (see Definition 10.5) to know that $\underline{\sigma}(\mathcal{D}) \perp\{\mathcal{E}, \mathcal{F}\}$ for each $\mathcal{E} \in \mathbf{P}_{\xi}^{\perp}$ and each $\mathcal{F} \in \mathbf{P}_{\sigma}^{\perp}$. Since $\mathbf{P}_{\sigma}^{\perp}$ is a copy (renamed in $\sigma$ ) of $\mathbf{P}_{\xi}^{\perp}$, we can also write this condition as

$$
\underline{\sigma}(\mathcal{D}) \perp\{\mathcal{E}, \mathcal{F}[\sigma / \xi]\}
$$

where both $\mathcal{F}$ and $\mathcal{E}$ vary in $\mathbf{P}_{\xi}^{\perp}$.

However, from Equation (6.1) we only have that $\underline{\sigma}(\mathcal{D}) \perp\{\mathcal{E}, \mathcal{E}[\sigma / \xi]\}$ : two copies of the same (up to renaming) strategy $\mathcal{E}$. This fact can be rephrased by saying that in our "HO setting", strategies in the type $\mathbf{P}^{\perp}$, which is, roughly speaking, "of the form $!_{N} \mathbf{C}$ " are uniform: every time we find a repeated action in $\mathbf{P}$, Opponent $\mathbf{P}^{\perp}$ reacts in the same way. 
6.4. A solution: non-uniform tests. The need for having enough tests appears similar to the one which has led Girard to the introduction of the daimon rule: in ludics, one typically opposes to an abstract "proof of $A$ " an abstract "counter-proof of $A$." To have enough tests (that is, to have both proofs of $A$ and proofs of $A^{\perp}$ ) there is a new rule which allow us to justify any premise.

Similarly here, when we oppose to a proof of $\mathbf{P}$ a proof of $\mathbf{P}^{\perp}$, we need enough counterstrategies. We are led to enlarge the universe of tests by introducing non-uniform counterstrategies. This is extremely natural to realize in an AJM setting [2, 4, where a strategy of type "! ${ }_{N} \mathbf{C}$ " is a sort of infinite tensor of strategies on "C", each one with its index of copy. To have HO non-uniform counter-strategies, we introduce a non-deterministic sum of strategies. Let us illustrate the idea, which we will formalize in the next section.

Non-uniform counter-strategies. The idea is to allow a kind of "non-deterministic sum" of negative strategies $\mathcal{E}, \mathcal{F}$. Let us, for now, informally write such a sum of $\mathcal{E}$ and $\mathcal{F}$ in the following way, which is reminiscent of non-deterministic sum in $\pi$-calculus, where the two possible (non-deterministic) choices are prefixed by $\tau$ actions:

$$
\tau . \mathcal{E}+\tau . \mathcal{F}
$$

- During the composition with other strategies, we might have to use several times this strategy, hence "entering" it several times. Every time is presented with this choice, normalization will non-deterministically choose one of the two possible continuations. The choice could be different at each repetition.

- To define orthogonality, we essentially set:

$\mathcal{D} \perp(\tau . \mathcal{E}+\tau . \mathcal{F})$ if and only if $\llbracket \mathcal{D}, \tau \cdot \mathcal{E}+\tau . \mathcal{F} \rrbracket$ is total for each possible choice among the $\tau$ 's.

It is immediate that:

$$
\mathcal{D} \perp(\tau . \mathcal{E}+\tau . \mathcal{F}) \Rightarrow \mathcal{D} \perp \mathcal{E} \text { and } \mathcal{D} \perp \mathcal{F} \text {. }
$$

As we will see, if $\mathcal{E} \in \mathbf{G}$ and $\mathcal{F} \in \mathbf{G}$ for $\mathbf{G}$ interpreting a formula of MELLS, we have that $(\tau . \mathcal{E}+\tau . \mathcal{F}) \in \mathbf{G}$, and vice-versa. Hence:

- if $\mathcal{D} \in \mathbf{P}$, for each $\mathcal{E}, \mathcal{F} \in \mathbf{P}^{\perp}$ we have $\mathcal{D} \perp(\tau . \mathcal{E}+\tau . \mathcal{F})$.

- By using Equation (6.1) we have that $\underline{\sigma}(\mathcal{D}) \perp\{(\tau . \mathcal{E}+\tau . \mathcal{F}),(\tau . \mathcal{E}+\tau . \mathcal{F})[\sigma / \xi]\}$.

- By using Equation (6.3), we deduce that $\underline{\sigma}(\mathcal{D}) \perp\{\mathcal{E}, \mathcal{F}[\sigma / \xi]\}$, as we wanted.

Linearity of the root. Observe that by construction, in $\underline{\sigma}(\mathcal{D})$ the action at the root is positive and it is the only action on the name $\sigma$. We can hence apply the same argument we have already given in Section 5.1 for the internal completeness of tensor.

As a consequence, if $P=?_{P}\left(N_{1} \otimes N_{2}\right)$, given a "good" strategy $\mathcal{D} \in \mathbf{P}_{\xi}$, we have that $\underline{\sigma}(\mathcal{D})$ actually belongs to $\vdash \mathbf{P}_{\sigma}, \mathbf{P}_{\xi}$ and $(\sigma,\{1,2\})^{+}$occurs linearly in $\underline{\sigma}(\mathcal{D})$ (only at the root). Hence, $\underline{\sigma}(\mathcal{D})$ can be decomposed in strategies $\underline{\sigma}(\mathcal{D})_{1} \in \vdash \mathbf{P}_{\xi}, \mathbf{N}_{\sigma 1}$ and $\underline{\sigma}(\mathcal{D})_{2} \in \vdash \mathbf{P}_{\xi}, \mathbf{N}_{\sigma 2}$.

This allows us to associate to $\mathcal{D} \in \vdash \mathbf{\Pi}, \mathbf{P}$ a proof which essentially has this form:

$$
\begin{gathered}
\vdots \\
\frac{\vdash \Pi, ?_{P}\left(N_{1} \otimes N_{2}\right), N_{1} \quad \vdash \Pi, ?_{P}\left(N_{1} \otimes N_{2}\right), N_{2}}{\frac{\vdash \Pi, ?_{P}\left(N_{1} \otimes N_{2}\right), N_{1} \otimes N_{2}}{\vdash \Pi, ?_{P}\left(N_{1} \otimes N_{2}\right)} \text { dereliction }+ \text { contraction }}
\end{gathered}
$$




\section{LUDICS WITH REPETITIONS: NON-UNIFORM STRATEGIES}

In this section we technically implement the ideas which we have presented in Section 6.4. In particular, we revise the definition of universal arena and strategy so to accommodate actions which corresponds to the $\tau$ actions we have informally introduced, and a notion of strategy which correspond to the $\tau$-sum. In this section we use ideas which have been developed to bridge between ludics and concurrency in [16], but here we choose a more standard presentation.

The silent arena. We extend the set of actions with a set $M_{\tau}=\{t\} \cup\left\{t_{i}: i \in \mathbb{N}\right\}$ of actions which we call silent. We define the arena $A_{\tau}=\left(M_{\tau}, \vdash, \lambda\right)$ as follows.

- the set of moves is $M_{\tau}$;

- the polarity is $\lambda(t)=+, \lambda\left(t_{i}\right)=-$;

- $t \vdash t_{i}$, for each $i$.

We can represent $A_{\tau}$ as follows:

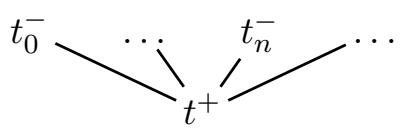

Definition 7.1 (Universal arena on an interface). Let $A(\xi, \epsilon)$ and $A_{\dagger}$ be as in Definition 4.1. We extend the construction of universal arena on an interface (Definition 4.3) as follows.

Let $\Pi=\xi_{1}, \ldots, \xi_{n}$ be a (possibly empty) positive interface.

The extended universal arena on the interface $\Pi$ is the arena

$$
U^{*}(\Pi):=A\left(\xi_{1},+\right)\|\cdots\| A\left(\xi_{n},+\right)\left\|A_{\dagger}\right\| A_{\tau} .
$$

The universal arena on a (negative) interface $\left\{\sigma^{-}\right\} \cup \Pi$ is the arena

$$
U^{*}(\Gamma):=A(\sigma,-) \triangleleft U^{*}(\Pi) .
$$

The silent actions play a role similar to that of $\dagger$ in Definition 4.6. The actions in both $A_{\dagger}$ and $A_{\tau}$ are "special actions" which are not localized on a name. Observe that even when the interface is empty, the universal arena still contains $A_{\uparrow} \| A_{\tau}$.

Non uniform strategies. As one may expected, we are now going to take strategies on $U^{*}(\Gamma)$. We reformulate the definition of strategy,

Definition 7.2 (Non-uniform strategies). Let $\Gamma$ be an interface. A non-uniform strategy (n.u. strategy for short) $\mathcal{D}$ on $\Gamma$, written $\mathcal{D}: \Gamma$, is a prefix-closed set of non-empty views (as in Definition 3.3) on the arena $U^{*}(\Gamma)$, such that:

(1) Coherence. If $s . m, s . n \in \mathcal{D}$ and $m \neq n$ then $m, n$ are negative.

(2) $\tau$-Positivity. If $s . m$ is maximal in $\mathcal{D}$ (i.e., no other view extends it), and $m$ is a proper action (i.e., and action on a name), then $m$ is positive.

We will call deterministic a n.u. strategy which has no silent actions. (Observe that in such a case the only special action is $\dagger$, and the definition coincides with the usual one, as in Definition 4.6.) 
The new Positivity condition says that if a maximal view terminates with a proper action, that action must be positive. However, a maximal view may terminate with a silent action $t_{i}$ (negative). This is necessary to our construction, and more precisely to the definition of orthogonality (see Section 9 and Example 9.3).

Remark 7.3. Definition 7.2 makes explicit the difference in role between proper actions and "special" actions, those in $A_{\dagger} \cup A_{\tau}$. This will be apparent in normalization. Since communication propagates along the names, the interaction between strategies only takes place on the proper actions. The $\dagger$ and the silent actions are never internal - as they have no names. But they have a fundamental role in the definition of orthogonality.

As a notational convention, from now on, by strategy we always mean n.u. strategy, otherwise we specify "deterministic" strategy.

Notation $\tau_{i}$. In the pictures, we will write $\tau_{i}$ for the segment of sequence $t . t_{i}$; this to convey the intuition that a pair $t . t_{i}$ represent a " $\tau$-action."

Example 7.4. The following is an example of non-uniform strategy:

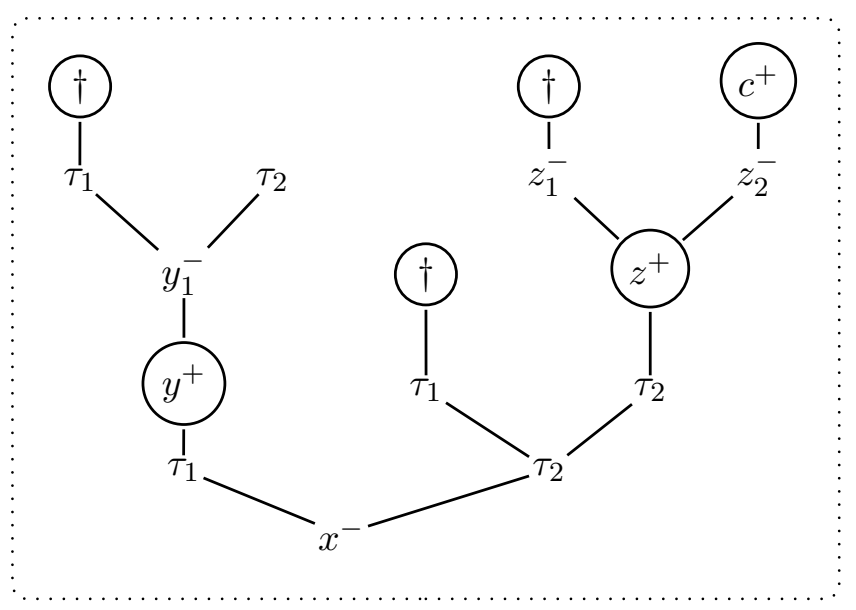

Sum of strategies. We use non-uniform strategies to capture the idea of "non-uniform" tests. As anticipated in Section 6.4, a non-uniform strategies can be seen as a non-deterministic sum of "standard" strategies.

Definition 7.5 ( $\tau$-sum). Given a family of strategies on the same interface (and hence all with the same polarity), we define their sum. Let $S$ be a non-empty subset of $\mathbb{N}$.

- If $\left\{\mathcal{D}_{i}: \Pi\right\}_{i \in S}$ is a family of positive strategies, we define their positive sum:

$$
\bigoplus_{i \in S}^{\tau} \mathcal{D}_{i}:=\bigcup_{i \in S}\left\{t^{+} \cdot t_{i}^{-} \cdot \mathcal{D}_{i}\right\}
$$

When $S$ is a finite set, say $\{1, \ldots, k\}$, we write $\mathcal{D}_{1} \oplus^{\tau} \cdots \oplus^{\tau} \mathcal{D}_{k}$.

- If $\left\{x^{-} . \mathcal{D}_{i}: \Gamma\right\}_{i \in S}$ is a family of negative strategies which have the same root $x^{-}$, we define their negative sum:

$$
\sum_{i \in S}^{\tau} x^{-} \cdot \mathcal{D}_{i}:=x^{-} \cdot \bigoplus_{i \in S}^{\tau} \mathcal{D}_{i}
$$

In the finite case, we also write $x^{-} \cdot \mathcal{D}_{1}+{ }^{\tau} \cdots+{ }^{\tau} x^{-} \cdot \mathcal{D}_{k}$. 
The following is easy to check.

Proposition 7.6. Let $\mathcal{D}_{i}$ be a family of positive strategies, and $\mathcal{F}_{i}$ a family of negative strategies. We have that $\bigoplus_{i \in S}^{\tau} \mathcal{D}_{i}$ is a positive strategy, and $\sum_{i \in S}^{\tau} \mathcal{F}_{i}$ is a negative strategy (in the sense of Definition [7.2).

A $\tau$-sum of strategies can be seen as a superposition of strategies in a way that they do not overlap.

Totality. Roughly speaking, a non-uniform strategy is total if it is not $\mathfrak{F i d}$, but also not obtained via $\tau$-sum with $\mathfrak{F i d}$. Precisely:

Definition 7.7 (Totality, non-uniform strategies). A strategy $\mathcal{D}$ is total if $\mathcal{D} \neq \mathfrak{F i d}$ and for each $s \in \mathcal{D}$, there are pointing sequences $p$ and $q$ such that:

$$
\text { p.a.q } \in \mathcal{D}, \quad s \sqsubseteq \text { p.a.q and } a \notin A_{\tau} .
$$

In words, each path from the root of $\mathcal{D}$ has to take to at least an action which is not silent. So for example, $\mathfrak{F i d} \oplus^{\tau} \mathfrak{F i d}$ or $\bigoplus_{1}^{\tau} \mathfrak{F i d}$ are partial strategies, as well as $\mathcal{D} \oplus^{\tau} \mathfrak{F i d}$, whatever positive strategy is $\mathcal{D}$. Notice also that any negative strategy is total, since it is either empty — and we have already discussed the reason of its totality in Section 4.1.1 — or it is not empty and each root is by definition a negative proper action (non-silent, because negative silent actions are never initial).

As in the linear case, in an untyped setting, partial strategies emerge naturally in case of unsuccessful interaction. In fact, they have a key role in the definition of orthogonality. Before discussing orthogonality and behaviours in the case of non-uniform strategies, we first make precise the definition of normalization.

Strategies on the empty interface. In the linear case, a strategy on the empty interface is a strategy on $A_{\dagger}$, hence, as we have already seen, there are only two possibilities: $\mathfrak{D a i}$ or Fid. The introduction of silent actions give more possibilities; a non-uniform strategy on the empty interface is a non-uniform strategy on the arena $A_{\dagger} \cup A_{\tau}$. Beside $\mathfrak{D a \mathfrak { i }}$ or $\mathfrak{F} \mathfrak{i d}$, there are other strategies, those which have as root $t^{+} \in A_{\tau}$. Each view can then contain several repetitions of silent actions, and terminate or not with a $\dagger$ action.

\section{Strategies With RePetitions: normalization Via the VAM abStract MACHINE}

The basic notions we use in this section (cut-net, visible action, ... ) are exactly the same as defined in Section 4.2 .

Composition of strategies in our setting works accordingly to the standard paradigm of "parallel composition plus hiding."

(1) Given a cut-net $\mathcal{R}$, we calculate the set of its interaction $I(\mathcal{R})$ (this is the parallel composition), which is a set of pointing strings, calculated via an abstract machine, the VAM [11].

(2) From the interactions $I(\mathcal{R})$, we obtain the strategy which corresponds to the normal form $\llbracket \mathcal{R} \rrbracket$ (Definition 8.9 ) by hiding the internal communication. 
Since we allow for the repetition of actions, there might be several occurrence of the same action $a$ in a strategy. To define the VAM, we need the following notion.

Definition 8.1 (View extraction). Let $s=x_{1} \ldots x_{n}$ be a pointing string of actions. We define the view of $s$ denoted by $\ulcorner s\urcorner$ as follows:

- $\ulcorner s\urcorner:=s$ if $s$ is empty;

- $\left\ulcorner s . x^{+}\right\urcorner:=\ulcorner s\urcorner \cdot x^{+}(x$ is positive);

- $\left\ulcorner s . x^{-}\right\urcorner=x^{-}$, if $x$ is initial (i.e., it does not point to any previous action).

- $\left\ulcorner s \cdot x^{-}\right\urcorner:=\ulcorner q\urcorner \cdot x^{-}$if $s=q . r . x$ and $x$ points to the last action of $q$.

Given a pointing string of actions $s=x_{1} \ldots x_{n}$ we obtain a subsequence $\ulcorner s\urcorner=x_{k_{1}} \ldots x_{k_{m}}$, where $1 \leq k_{1}<\cdots<k_{m} \leq n$. We say that the element at position $j$ in $\ulcorner s\urcorner(1 \leq j \leq m)$ corresponds to the element at position $k_{j}$ in $s\left(1 \leq k_{j} \leq n\right)$. We will use this in the following.

As for the pointers, the operation \ulcorner\urcorner is pointer preserving in the sense that if $x_{k_{y}}, x_{k_{z}}$ in $\ulcorner s\urcorner$ respectively correspond to $x_{i}, x_{j}$ in $s$, and $x_{j}$ points to $x_{i}$ in $s$, then $x_{k_{z}}$ points to $x_{k_{y}}$ in $\ulcorner s\urcorner$.

In words, we trace back from the end of $s$ : (i) following the pointers of negative actions of $s$ and erasing all actions under such pointers, (ii) bypassing positive actions, (iii) stopping the process when we reach an initial negative action. 4

Example 8.2. Given $s=x_{1} \cdot x_{2} \ldots x_{6}$ and $t=y_{1} \cdot y_{2} \ldots y_{5}$ as follows:

$$
s=a^{+} a^{-} b^{+} b_{0}^{-} a_{0}^{+} a_{0}^{-} \quad t=a^{+} a^{-} b^{+} b_{0}^{-} a_{0}^{+}
$$

we get $\ulcorner s\urcorner=x_{1} \cdot x_{6}$ and $\ulcorner t\urcorner=y_{2} \cdot y_{3} \cdot y_{4} \cdot y_{5}$ :

$$
\ulcorner s\urcorner=a^{+} a_{0}^{-} \quad\ulcorner t\urcorner=a^{-} b^{+} b_{0}^{-} a_{0}^{+}
$$

We will also rely on the following Lemma.

Lemma 8.3. Let $\mathcal{R}=\left\{\mathcal{D}_{1}, \ldots, \mathcal{D}_{n}\right\}$ be a cut-net. The following properties hold:

(1) Given an address $\xi$, and a polarity $\epsilon, \xi^{\epsilon}$ occurs in at most one of the interfaces.

(2) Each view $s \in \bigcup\left\{\mathcal{D}_{i}: 1 \leq i \leq n\right\}$ belongs exactly to one $\mathcal{D}_{i}$.

Proof.

(1) By definition of cut-net (Definition 4.11).

(2) The first action $x$ in the view $s$ is enough to determine to which $\mathcal{D}_{i}$ it belongs. If $x$ is a proper action $(\xi, I)^{\epsilon}$, the conclusion is immediate from the previous point ( $x$ belongs to the same strategy to which $\xi^{\epsilon}$ belongs). If $x$ is in $A_{\dagger} \cup A_{\tau}$, the interface must be positive; we already observed that a cut-net contains at most one positive interface.

\footnotetext{
${ }^{4}$ In general, the procedure of view extraction may delete some pointers. This will never happen for the pointing strings we consider in this paper.
} 
8.1. VAM (View-Abstract-Machine). Let us first informally explain how the abstract machine calculates the interaction of a cut-net $\mathcal{R}$. The machine visits actions of the strategies in $\mathcal{R}$ and collects the sequences of visited actions, proceeding as follows:

- We start on the roots of the main strategy of a cut-net $\mathcal{R}$.

- If we visit a visible action $a$ occurring in some $\mathcal{D} \in \mathcal{R}$, we continue to explore the current strategy $\mathcal{D}$. The process branches when $a$ is a branching node of $\mathcal{D}$.

- If we visit an internal action $a^{+}$occurring in $\mathcal{D}$ we match it with its opposite $a^{-}$occurring in $\mathcal{E} \in \mathcal{R}$, then we continue to collect actions in $\mathcal{E}$ (this is a jump of the machine). Since there could be several occurrences of $a^{-}$in $\mathcal{E}$, we use \ulcorner\urcorner to determine the correct occurrence of action to which we have to move.

- We may eventually stop when either we reach a maximal action or an internal action which has no match.

We now give the formal definition of the VAM. The definition below is a reformulation of the machine defined in [11].

Definition 8.4 (VAM). Let $\mathcal{R}=\left\{\mathcal{D}_{1}, \ldots, \mathcal{D}_{n}\right\}$ be a cut-net. $V A M(\mathcal{R})$ is the set pointing strings defined as follows. The construction preserves the following invariant:

$\left(^{*}\right) \quad$ if $p \in V A M(\mathcal{R})$ then $\ulcorner p\urcorner$ is a view which belongs to one of the $\mathcal{D} \in \mathcal{R}$.

(1) (Initialization) If the main strategy of $\mathcal{R}$ is empty, we set $V A M(\mathcal{R}):=\emptyset$. Otherwise, for each $a$ root of the main strategy, $a \in V A M(\mathcal{R})$.

(2) Let $p=x_{1} \ldots x_{n} \in V A M(\mathcal{R})$. We have the following two cases.

(a) (Continuation)

The action $x_{n}$ is either a negative action or a positive visible action. We consider $\ulcorner p\urcorner$. There exists a unique strategy $\mathcal{D} \in \mathcal{R}$ such that $\ulcorner p\urcorner \in \mathcal{D}$ (because of the invariant $(*)$ and Lemma 8.3). For each action $a$ which extends $\ulcorner p\urcorner$ in $\mathcal{D}$ (i.e., such that $\ulcorner p\urcorner . a \in \mathcal{D}$ ) we set $p . a \in V A M(\mathcal{R})$ where the pointer for $a$ is given by the equation $\ulcorner p . a\urcorner=\ulcorner p\urcorner . a$.

(b) (Jump) The action $x_{n}=a^{+}$is an internal positive action. We consider the sequence $p . a^{-}$obtained by adding the action $a^{-}$to $p$ and possibly a pointer as follows. If $x_{n}=(\xi i, J)^{+}$points to $x_{i}=(\xi, I)^{-}$we add a pointer from $a^{-}$to $x_{i-1}$ in $p$. If there is $\mathcal{D} \in \mathcal{R}$ such that $\left\ulcorner p . a^{-}\right\urcorner \in \mathcal{D}$, we set $p . a^{-} \in V A M(\mathcal{R})$. That occurrence of $a^{-}$is the match of $a^{+}$.

Remark 8.5 (The pointers in case (2)). In the case (2)(a), the equation $\ulcorner p \cdot a\urcorner=\ulcorner p\urcorner \cdot a$ summarizes the following conditions.

- Assume $a$ is negative. $a$ must point to $x_{n}$.

- Assume $a$ is positive. Let $\ulcorner p\urcorner=x_{k_{1}} \ldots x_{k_{m}}$, where each $x_{k_{j}}$ corresponds to en element $x_{i}$ in $p$. If in the view $\ulcorner p\urcorner . a \in \mathcal{D}$ we have that $a$ points to $x_{k_{j}}$, in $p . a$, we have that $a$ points to the corresponding element.

In the case $(2)(b)$, we have the following distinct situations.

- If $x_{n}=(\xi i, J)^{+}$points to $x_{i}=(\xi, I)^{-}$, then $i>1$, because if $x_{i}$ is a negative hidden action and by definition of cut-net it cannot be a root of the main strategy of $\mathcal{R}$. (Moreover, it is easily seen that $x_{i-1}=(\xi, I)^{+}$so that $x_{i}$ has been introduced by a Jump case.)

- If $x_{n}=(\xi, J)^{+}$points to a negative action $x_{i}=(\sigma, K)^{-}$with $\sigma$ and $\xi$ disjoint, it means that both occurrences of actions belongs to the same strategy $\mathcal{D} \in \mathcal{R}$ of interface $\sigma^{-}, \xi^{+}, \Pi$. 
We do not need to add any pointer, because the match for $x_{n}$ (if any) must be a root of a strategy $\mathcal{E}: \xi^{-}, \Pi^{\prime}$ of $\mathcal{R}$.

- Last, if $x_{n}=(\xi, J)^{+}$does not point to any action, then $x_{n}$ occurs in a strategy $\mathcal{D} \in \mathcal{R}$ of interface $\xi^{+}, \Pi$. Again, we do not need to add any pointer, because the match for $x_{n}$ (if any) must be a root of a strategy $\mathcal{E}: \xi^{-}, \Pi^{\prime}$ of $\mathcal{R}$.

Remark 8.6. Observe that the interface $\Gamma$ of the cut-net $\mathcal{R}$ is negative if and only if the main strategy is negative. In such a case, the first action in each $p \in V A M(\mathcal{R})$ is a root $a^{-}$ of the main strategy. Such an action is visible, and never occurs again in $p$.

\subsection{Normal form.}

Definition 8.7 (Hiding). Given a cut-net $\mathcal{R}$, and $p=x_{1} \ldots x_{n} \in V A M(\mathcal{R})$, we define $\operatorname{hide}(p)$ as the pointing string obtained as follows:

(1) If $x_{1}=a^{-}$is negative (see the remark above), the pointers are updated as follows: for each $c^{+}$visible action pointing to an internal action $b^{-}$, we make $c^{+}$point to $x_{1}$.

(2) We hide all the internal actions.

All pointers between actions which are visible are preserved, i.e., if $p=x_{1} \ldots \ldots x_{n}$ and $x_{i}$ points to $x_{j}(1 \leq j<i \leq n)$ in $p$, and both $x_{i}, x_{j}$ are visible, then in hide $(p)$ the action (which corresponds to) $x_{i}$ still points to the action (which corresponds to) $x_{j}$.

What remains after hiding is the subsequence of visible actions of $p$, written hide $(p)$, with the inherited pointer structure. If $X \subseteq V A M(\mathcal{R})$, we will also write hide $(X)$ for the set $\{\operatorname{hide}(p): p \in X\}$.

Remark 8.8. Observe that after step (1) of hiding, each visible action in $p$ either does not point to any previous action (but only because it is initial in the arena induced by the interface of the cut-net) or it points to a visible action. The latter point deserves some explanations. If an action $c^{+}$is visible and points to an internal action (say $b^{-}$), it means that both $b^{-}$and $c^{+}$belong to the same strategy $\mathcal{D}: \xi^{-}, \Pi$. Moreover, by definition of internal action and of the universal arena $U^{*}\left(\xi^{-}, \Pi\right)$, the action $b^{-}$must be an action $(\xi, I)^{-}$, initial in $A\left(\xi^{-}\right)$, the action $c^{+}$must be initial in $U^{*}(\Pi)$, and the pointer form $c^{+}$to $b^{-}$must correspond to the enabling $b^{-} \vdash c^{+}$introduced by the construction $A(\xi,-) \triangleleft U^{*}(\Pi)$.

By using the $V A M$ machine, we are now going to define the set $I(\mathcal{R})$ of the interactions of a cut-net $\mathcal{R}$, and from this its normal form $\llbracket \mathcal{R} \rrbracket$.

Definition 8.9 (Normal form). Let $\mathcal{R}$ be a cut-net. We define the set $I(\mathcal{R})$ of the interactions of $\mathcal{R}$ as the closure under non-empty prefix of

$$
\{q . c \in V A M(\mathcal{R}): c \text { is visible and not a proper negative action }\} \text {. }
$$

The normal form of $\mathcal{R}$, denoted by $\llbracket \mathcal{R} \rrbracket$ is defined as

$$
\llbracket \mathcal{R} \rrbracket=\{\operatorname{hide}(p): p \in I(\mathcal{R}) \text { and hide }(p) \text { non-empty }\} .
$$

Observe that since $V A M(\mathcal{R})$ is closed by non-empty prefix, we have that $I(\mathcal{R}) \subseteq V A M(\mathcal{R})$.

The normal form of a cut-net is a strategy. We show this fact in Proposition 8.12

Lemma 8.10. If p. $m, p . n \in V A M(\mathcal{R})$ and $m \neq n$, then $m, n$ are negative and visible. 
Proof. First of all, we observe that the polarity of $m$ and $n$ is the same. Assume $m, n$ are positive. If $p$ is empty, then $m, n$ are both roots of the main strategy (Case (1) in the VAM). By Coherence condition (Definition 7.2 ), we have that $m=n$. The case of $p$ non-empty is similar, with $m, n$ which extend $\ulcorner p\urcorner$ (Case (2)(a) in the VAM).

We have established that if $m \neq n$, then $m, n$ are negative. They must also be visible, because the only way to extend $p$ with a negative, internal action is Case (2)(b). However, in such a case since the extension of $\ulcorner p\urcorner$ is uniquely given by the construction, we would have that $m=n$.

Corollary 8.11. Let $p, q \in V A M(\mathcal{R})$. If hide $(p)=$ hide $(q)$ then $p \sqsubseteq q$ or $q \sqsubseteq p$.

Proof. Assume that neither $p \sqsubseteq q$ nor $q \sqsubseteq p$ hold, and let $s$ be the longest common prefix of $p$ and $q$, i.e., s.a $a p, s . b \sqsubseteq q$, and $a \neq b$. By Proposition 8.10, $a, b$ are negative and visible, hence hide $(s . a) \neq$ nide $(s . b)$ which contradicts the fact that hide $(p)=\operatorname{hide}(q)$.

Proposition 8.12. If $\mathcal{R}$ is a cut-net of interface $\Gamma$, then $\llbracket \mathcal{R} \rrbracket$ is a strategy on $\Gamma$.

Proof. We show the following:

(1) if $p \in V A M(p)$, then hide $(p)$ is a view (on the arena $U^{*}(\Gamma)$ );

(2) the set hide $(\operatorname{VAM}(\mathcal{R}))$ satisfies the Coherence condition (as given in Definition 7.2);

(3) $I(\mathcal{R})$ and $\llbracket \mathcal{R} \rrbracket$ satisfy $\tau$-Positivity (as given in Definition 7.2 ).

(1) a. $\operatorname{hide}(p)$ is a justified sequence (on the appropriate arena). It is immediate to check that the pointers in hide( $p)$ satisfy the justification condition, as this fact is inherited from the same property of the strategies which take part in the construction of $p$. The only delicate aspect are the pointers in the case where the interface of $\mathcal{R}$ is negative, i.e., $\Gamma=\sigma^{-}, \Pi$. More precisely, the pointers which in the arena $U^{*}\left(\sigma^{-}, \Pi\right)=A(\sigma,-) \triangleleft U^{*}(\Pi)$ correspond to the enabling $a \vdash c$, with $a$ initial in $A(\sigma,-)$ and $c$ initial in $U^{*}(\Pi)$. However, this situation is taken care by step (1) in the hiding (the updating of the pointers, see Remark 8.8).

b. $\operatorname{hide}(p)$ is alternating. First of all, we observe that if $p \in V A M(\mathcal{R})$, then $p$ is alternating (by construction). As a consequence, $h i d e(p)$ is also alternating, because all occurrences of internal actions appear (and are deleted) as pairs $p=p^{\prime} \cdot a^{+} \cdot a^{-} \cdot p^{\prime \prime}$, or they occur in the very last position.

c. $\operatorname{hide}(p)$ is a view. Each visible negative action in $p$ is either initial (Case (1)), or points to its immediate predecessor (Case (2)(a)).

(2) Assume $s . m \neq s . n$. We have that $s . m=\operatorname{hide}(p . m)$, s.n $=\operatorname{hide}(q . n)$ and $\operatorname{hide}(p)=$ hide $(q)=s$. Using Corollary 8.11, let assume $p \sqsubseteq q$. Let us consider $p . x \sqsubseteq q . n$. By Lemma 8.10, we have that if $m \neq x$, then $m, x$ are negative. The execution of the VAM introduces both $p . m$ and $p . x$ by checking how $\ulcorner p\urcorner$ is extended in $\mathcal{R}$. Since $m$ is visible, $x$ must be as well. From this we deduce that $x=n$ and then that $p=q$. Hence, $m, n$ are negative, by Lemma 8.10 ,

(3) We first observe that the $\tau$-Positivity condition holds in $I(\mathcal{R})$. Assume that $p=q . c \in$ $I(\mathcal{R})$ and has no extensions in $I(\mathcal{R})$; then $c$ is not a proper negative action. Moreover, from the fact that $c$ is visible, it follows that $\llbracket \mathcal{R} \rrbracket$ satisfies $\tau$-Positivity.

Like for the strategies in [22], our strategies are a variant of abstract Böhm trees (see [9, 32 for a description of ludics strategies in terms of Böhm trees). Abstract Böhm trees normalize via the VAM abstract machine [8, 11], which we have described in this section; a fundamental property that normalization satisfies is associativity. 
Theorem 8.13 (Associativity). Let $\mathcal{R}$ be a cut-net which can be partitioned into cut-nets $\mathcal{R}=\mathcal{R}_{1}, \ldots, \mathcal{R}_{n}$. We have:

$$
\llbracket \mathcal{R} \rrbracket=\llbracket \llbracket \mathcal{R}_{1} \rrbracket, \ldots, \llbracket \mathcal{R}_{n} \rrbracket \rrbracket
$$

We omit the formal proof of this fact, which is established in [8, 11]. We must observe that in our setting, the only essential difference (w.r.t. abstract Böhm trees) are the conditions on the polarity of the maximal actions, but this is irrelevant to establish associativity.

8.3. Renamings and normalization. We now state a property which relates renamings (in the sense of Section 6.1) and normalization. We first generalize the renaming operator defined in Section 6.1. We now allow simultaneous renamings in arbitrary interfaces.

Let $\Gamma=\left\{\alpha_{1}^{\epsilon_{1}}, \ldots, \alpha_{k}^{\epsilon_{k}}, \alpha_{k+1}^{\epsilon_{k+1}}, \ldots, \alpha_{n}^{\epsilon_{n}}\right\}$ be an interface, $\Delta=\left\{\alpha_{1}^{\epsilon_{1}}, \ldots, \alpha_{k}^{\epsilon_{k}}\right\}$ and $\Delta^{\prime}=$ $\beta_{1}, \ldots, \beta_{k}$ names such that $\Gamma^{\prime}:=\left\{\beta_{1}^{\epsilon_{1}}, \ldots, \beta_{k}^{\epsilon_{k}}\right\} \cup\left\{\alpha_{k+1}^{\epsilon_{k+1}}, \ldots, \alpha_{n}^{\epsilon_{n}}\right\}$ forms an interface.

Let $\mathcal{D}$ be a strategy on $\Gamma$. By $\mathcal{D}\left[\Delta^{\prime} / \Delta\right]$ we denote the strategy on interface $\Gamma^{\prime}$ obtained from $\mathcal{D}$ by renaming, in all occurrences of action, the prefix $\alpha_{l}$ into $\beta_{l}$ for any $1 \leq l \leq k$. We observe that $\mathcal{D}\left[\Delta^{\prime} / \Delta\right]$ is indeed a strategy (in the sense of Definition [7.2) because:

(1) we explicitly request that $\Gamma^{\prime}$ forms an interface;

(2) the conditions of being a strategy are inherited from $\mathcal{D}$, since the operation of renaming preserves the polarity of the actions, their nature (proper, silent, ...), and the pointer structure of the strategy (our operation acts only on names of proper actions, all the existing pointers are preserved).

The main property we need in the sequel is following one. Let us consider the following data:

- $\mathcal{D}: \Gamma, \xi^{+}, \sigma^{+}$

- $\mathcal{E}: \xi^{-}, \Delta$, where $\Delta=\alpha_{1}^{+}, \ldots, \alpha_{k}^{+}$;

- $\mathcal{E}\left[\sigma / \xi, \Delta^{\prime} / \Delta\right]: \sigma^{-}, \Delta^{\prime}$, where $\Delta^{\prime}=\beta_{1}^{+}, \ldots, \beta_{k}^{+}$(an "isomorphic" copy of $\mathcal{E}$ );

- $\mathcal{D}[\xi / \sigma]: \Gamma, \xi^{+}$;

- $\left(\mathcal{F}_{j}\right)=\mathcal{F}_{1}, \ldots, \mathcal{F}_{n}$ a list of strategies.

Let us assume that $\mathcal{R}:=\left\{\mathcal{D}, \mathcal{E}, \mathcal{E}\left[\sigma / \xi, \Delta^{\prime} / \Delta\right],\left(\mathcal{F}_{j}\right)\right\}$ forms a cut-net of interface $\Lambda, \Delta, \Delta^{\prime}$, where names in $\Lambda$ comes from $\Gamma$ and the names of the interfaces of $\left(\mathcal{F}_{j}\right)$, so that $\mathcal{R}^{\prime}:=$ $\left\{\mathcal{D}[\xi / \sigma], \mathcal{E},\left(\mathcal{F}_{j}\right)\right\}$ is a cut-net on interface $\Lambda, \Delta$. We have:

\section{Proposition 8.14.}

$$
\llbracket \mathcal{D}, \mathcal{E}, \mathcal{E}\left[\sigma / \xi, \Delta^{\prime} / \Delta\right],\left(\mathcal{F}_{j}\right) \rrbracket\left[\Delta / \Delta^{\prime}\right]=\llbracket \mathcal{D}[\xi / \sigma], \mathcal{E},\left(\mathcal{F}_{j}\right) \rrbracket .
$$

Proof. (Sketch.) By reasoning on the names of the interfaces, we can easily deduce:

- The names in $\Delta$ and $\Delta^{\prime}$ (resp. $\Delta$ ) are not cuts in $\mathcal{R}$ (resp. $\mathcal{R}^{\prime}$ ).

- $\mathcal{D}$ (resp. $\mathcal{F}_{j}$ ) is the main strategy of $\mathcal{R}$ if and only if $\mathcal{D}[\xi / \sigma]$ (resp. $\mathcal{F}_{j}$ ) is the main strategy of $\mathcal{R}^{\prime}$.

- Neither $\mathcal{E}$ nor $\mathcal{E}\left[\sigma / \xi, \Delta^{\prime} / \Delta\right]$ (resp. $\mathcal{E}$ ) is the main strategy of $\mathcal{R}$ (resp. $\mathcal{R}^{\prime}$ ).

If we normalize $\mathcal{R}$ and $\mathcal{R}^{\prime}$, they share a very similar dynamics: the only difference is that the part of interaction between $\mathcal{D}, \mathcal{E}$ and the "isomorphic" copy of $\mathcal{E}$ in $\mathcal{R}$ is reproduced in $\mathcal{R}^{\prime}$ by $\mathcal{D}[\sigma / \xi]$ and $\mathcal{E}$. But this creates no relevant differences on the pointing string in $V A M(\mathcal{R})$ and $V A M\left(\mathcal{R}^{\prime}\right)$, since we have a pointing string e.g.,

$$
p=\ldots(\sigma, I) \ldots(\xi . \gamma, K) \ldots\left(\alpha_{i} . L\right) \ldots\left(\beta_{j} . M\right) \ldots \in V A M(\mathcal{R})
$$


if and only if we have a "corresponding" pointing string of the same length

$$
p^{\prime}=\ldots(\xi, I) \ldots(\xi \cdot \gamma, K) \ldots\left(\alpha_{i} . L\right) \ldots\left(\alpha_{j} . M\right) \ldots \in V A M\left(\mathcal{R}^{\prime}\right)
$$

where, for $p=x_{1} \ldots x_{n}$ and $p^{\prime}=y_{1} \ldots y_{n}$ :

- the polarity of $x_{i}$ and $y_{i}$ is the same, for $1 \leq i \leq n$;

- the pointer structures of $p$ and $p^{\prime}$ are exactly the same in the sense that $x_{i}$ points to $x_{j}$ if and only if $y_{i}$ points to $y_{j}$;

- for any $1 \leq i \leq n$, either $x_{i}=y_{i}$ or $x_{i}=(\sigma \cdot \gamma, I)$ and $y_{i}=(\xi \cdot \gamma, I)$ or $x_{i}=\left(\alpha_{l} \cdot \gamma, I\right)$ and $y_{i}=\left(\beta_{l} \cdot \gamma, I\right)$, for $1 \leq l \leq k$.

This follows from the fact that using the views extraction operation on pointing strings given by the VAM, we can univocally determine a specific view of strategy. In particular, given $p$ (resp. $p^{\prime}$ ) we can univocally reconstruct $p^{\prime}$ (resp. $p$ ).

Since the nature (visible, hidden, proper, silent,...) of the action in $p$ and $p^{\prime}$ corresponds elementwise too, it follows that $p \in I(\mathcal{R})$ if and only if there is a "corresponding" $p^{\prime} \in I\left(\mathcal{R}^{\prime}\right)$. The difference between $p$ and $p^{\prime}$ then is only on names occurring in proper actions, but names generated by cuts $\xi$ and $\sigma$ will be erased by hiding, thus obtaining a view $s \in \llbracket \mathcal{R} \rrbracket$ and a "corresponding" view $s^{\prime} \in \llbracket \mathcal{R}^{\prime} \rrbracket$, and names generated by $\Delta^{\prime}$ which are not in $s^{\prime}$ are renamed by using the renaming $\left[\Delta / \Delta^{\prime}\right]$ which is only needed to this aim.

A formal proof can be carried out by induction on the length of the pointing strings.

As a special instance of the previous proposition we have:

Proposition 8.15 (Copies). Let $\mathcal{D}: \xi^{+}$and $\mathcal{E}: \xi^{-}$be strategies. We have:

$$
\llbracket \mathcal{D}, \mathcal{E} \rrbracket=\llbracket \underline{\sigma}(\mathcal{D}), \mathcal{E}, \mathcal{E}[\sigma / \xi] \rrbracket .
$$

\section{Orthogonality And interactive types}

9.1. Orthogonality. The definition of orthogonality is the same as for linear strategies (Definition 4.31); we repeat it for convenience.

Like in the linear case (and with a similar meaning) orthogonality is a relation which is defined on total strategies (with the notion of totality being now that in Definition 7.7).

Definition 9.1 (Orthogonality, orthogonal set). Let $\mathcal{D}: \Gamma$ be a total strategy and $\left(\mathcal{E}_{\xi}\right)_{\xi \in \Gamma}$ be a family of counter-strategies (Definition 4.29). $\mathcal{D}$ and $\left(\mathcal{E}_{\xi}\right)$ are said to be orthogonal, written $\mathcal{D} \perp\left(\mathcal{E}_{\xi}\right)$ if $\llbracket \mathcal{D},\left(\mathcal{E}_{\xi}\right) \rrbracket$ is total.

Given a set $\mathbf{S}$ of total strategies on the same interface $\Gamma$, its orthogonal set is defined as

$\mathbf{S}^{\perp}:=\left\{\left(\mathcal{E}_{\xi}\right):\left(\mathcal{E}_{\xi}\right)\right.$ is a family of counter-strategies w.r.t. $\Gamma$ and $\left(\mathcal{E}_{\xi}\right) \perp \mathcal{D}$ for any $\left.\mathcal{D} \in \mathbf{S}\right\}$.

Similarly, given a set $\mathbf{C}$ of families of counter-strategies w.r.t. $\Gamma$, its orthogonal set is defined as

$$
\mathbf{C}^{\perp}:=\left\{\mathcal{D}: \mathcal{D} \text { is total on interface } \Gamma \text { and } \mathcal{D} \perp\left(\mathcal{E}_{\xi}\right) \text { for any }\left(\mathcal{E}_{\xi}\right) \in \mathbf{C}\right\} .
$$


Observe that $\llbracket \mathcal{D},\left(\mathcal{E}_{\xi}\right) \rrbracket$ is a strategy on the empty interface. By Definition 7.7 , we have orthogonality if $\llbracket \mathcal{D},\left(\mathcal{E}_{\xi}\right) \rrbracket \neq \mathfrak{F i d}$ and for each $s \in \llbracket \mathcal{D},\left(\mathcal{E}_{\xi}\right) \rrbracket$ there is $p . \dagger \in \llbracket \mathcal{D},\left(\mathcal{E}_{\xi}\right) \rrbracket$ such that $s \sqsubseteq p . \dagger$. The intuition is that each interaction should lead to $\dagger$. Coherently with this intuition, we will also see that two distinct views in the normal form of a closed net always branch on silent actions.

The following lemma is a direct consequence of the definition of orthogonality:

Lemma 9.2. Let $\mathcal{E}: \xi^{-}$be a negative strategy such that $\mathcal{E}=\sum_{i \in S}{ }^{\tau} \mathcal{E}_{i}$. Let $\left\{\mathcal{D}_{1}, \ldots, \mathcal{D}_{n}, \mathcal{E}\right\}$ be a closed cut-net. We have that:

$$
\llbracket \mathcal{D}_{1}, \ldots, \mathcal{D}_{n}, \mathcal{E} \rrbracket \text { is total } \Rightarrow \llbracket \mathcal{D}_{1}, \ldots, \mathcal{D}_{n}, \mathcal{E}_{i} \rrbracket \text { is total, for each } i \in S .
$$

Hence in particular, for any strategy $\mathcal{D} \perp \mathcal{E}$, we have

$$
\mathcal{D} \perp \mathcal{E} \Rightarrow \mathcal{D} \perp \mathcal{E}_{i}, \quad \text { for each } i \in S .
$$

The converse of Lemma 9.2 does not hold in general. We now give a concrete example, which is also useful to better understand composition and orthogonality.

Example 9.3. Let us consider the following strategies.

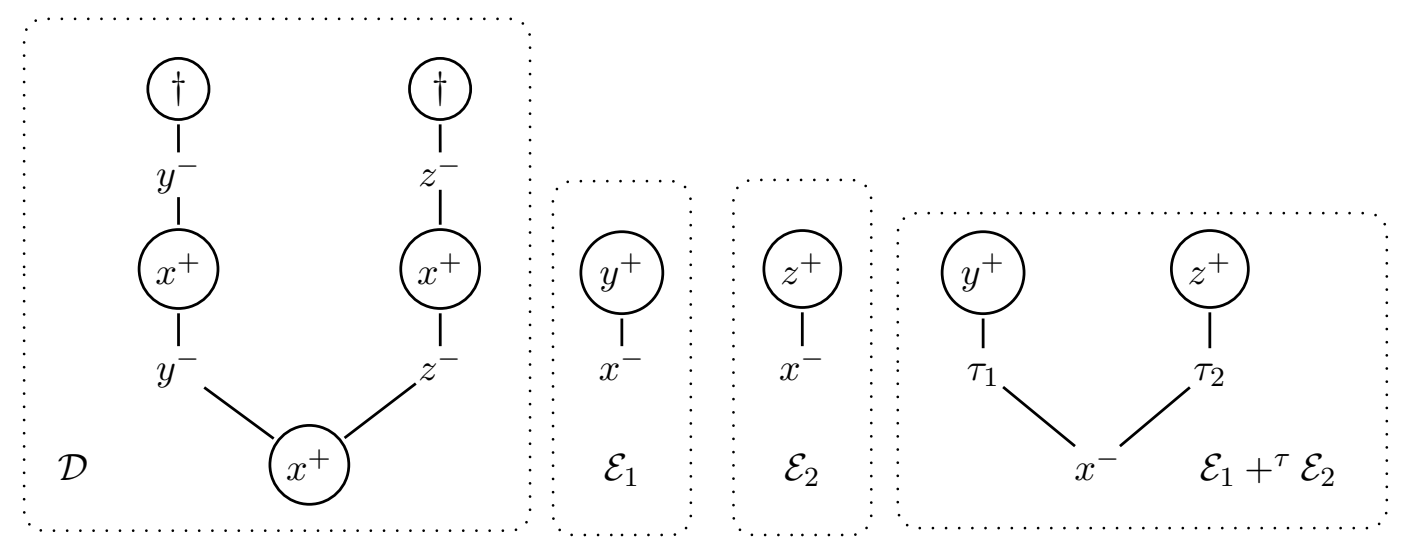

If we compose $\mathcal{D}$ with $\mathcal{E}_{i}$, it is rather clear that we always reach $\dagger$, hence $\mathcal{D} \perp \mathcal{E}_{1}$ and $\mathcal{D} \perp \mathcal{E}_{2}$. On the other hand, if we compose $\mathcal{D}$ with $\mathcal{E}_{1}+{ }^{\tau} \mathcal{E}_{2}$, we have the interaction as (partially) described below.

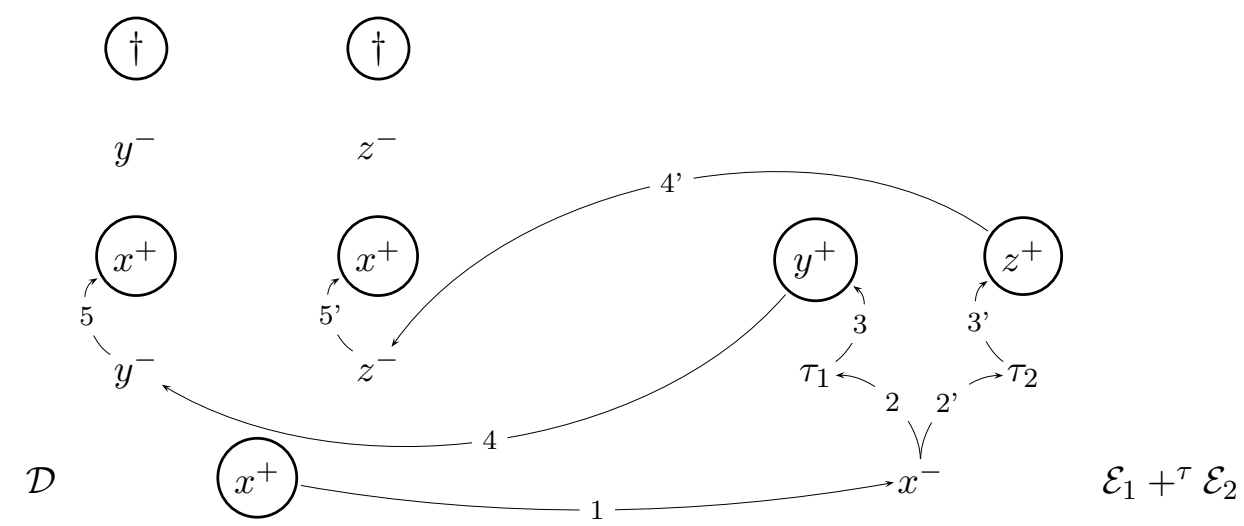


After the steps tagged by 5 and $5^{\prime}$ the interaction "re-enters" in $\mathcal{E}_{1}+{ }^{\tau} \mathcal{E}_{2}$. The steps which follow 5 are described below (for the steps which follow $5^{\prime}$ the situation is symmetric).

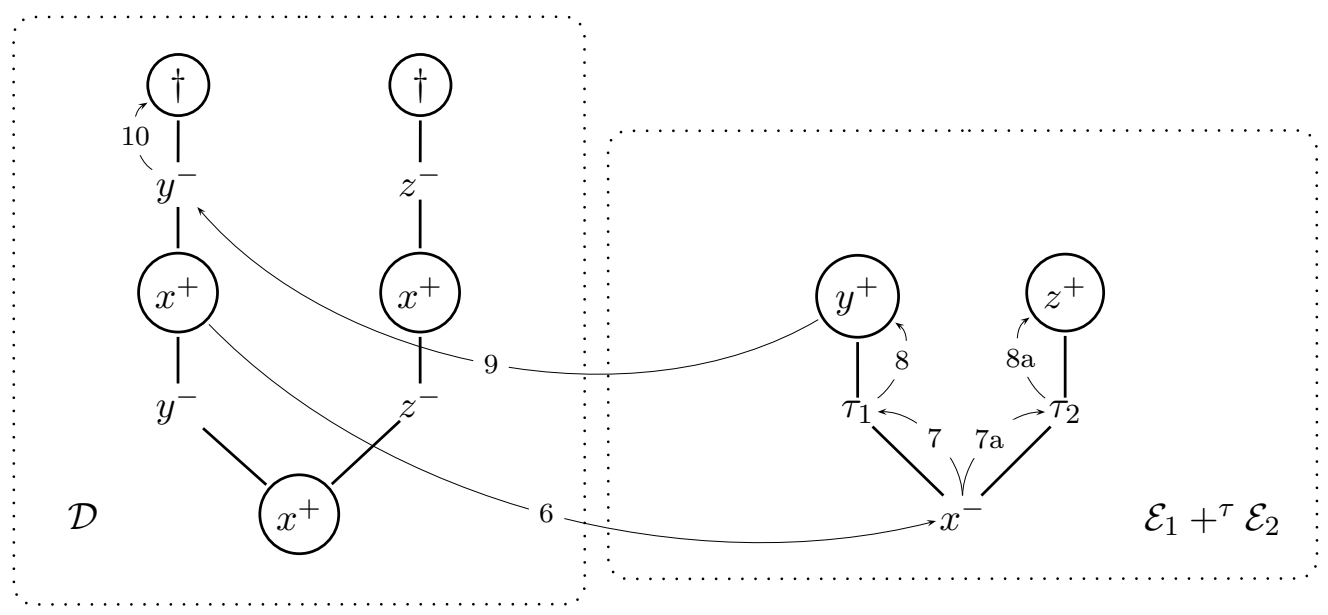

Notice that after the step tagged by $8 a$ we have a deadlock: the action $z^{+}$should match an action $z^{-}$above (i.e., justified) by the last visited occurrence of $x^{+}$(the leftmost one), but there is no such an action since we only have $y^{-}$.

The result of composition is:

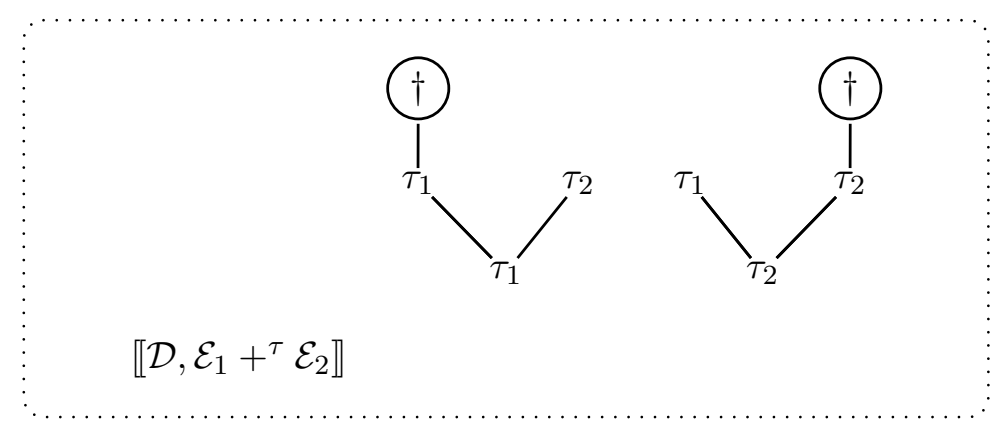

which has four maximal views:
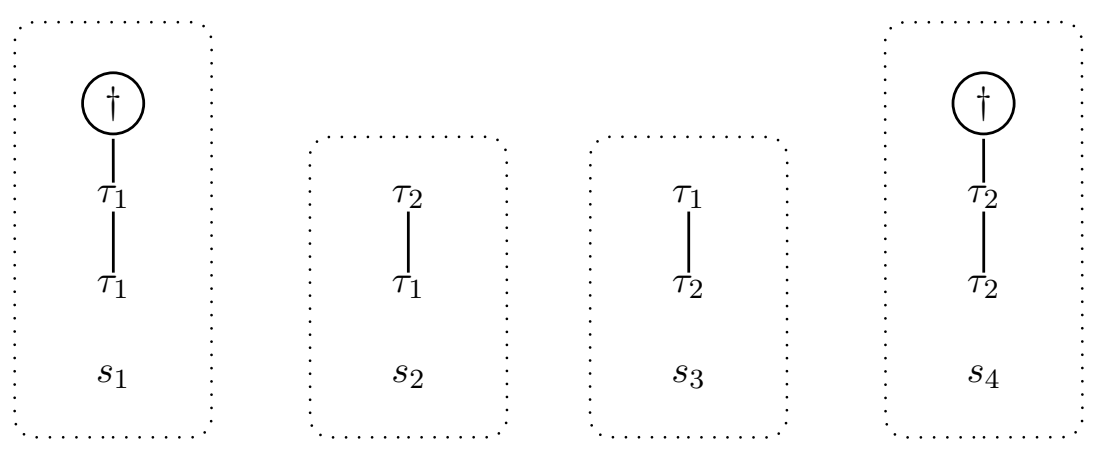
From this we conclude that $\mathcal{D} \not \perp \quad \mathcal{E}_{1}+{ }^{\tau} \mathcal{E}_{2}$ because $s_{2}$ and $s_{3}$ do not satisfy the totality condition of Definition 7.7 .

We will use also the following properties of normalization.

Lemma 9.4. Let $\mathcal{D}, \mathcal{D}_{1}, \mathcal{D}_{2}$ be strategies on the same positive interface $\Pi=\xi 1^{+}, \ldots, \xi n^{+}$. Let $\left(\mathcal{E}_{i}\right)_{\xi i \in \Pi}=\left(\mathcal{E}_{i}\right)=\left\{\mathcal{E}_{1}, \ldots, \mathcal{E}_{n}\right\}$ be a family of (negative) counter-strategies w.r.t. $\Pi$.

We have the following:

(a) $\left\{\mathcal{E}_{1}, \ldots, \mathcal{E}_{n}\right\} \perp \mathcal{D}$ if and only if $x^{+} .\left\{\mathcal{E}_{1}, \ldots, \mathcal{E}_{n}\right\} \perp x^{-}$.D , where $x=\left(\xi, I_{n}\right)$;

(b) $\left(\mathcal{E}_{i}\right) \perp \mathcal{D}_{1} \oplus^{\tau} \mathcal{D}_{2}$ if and only if $\left(\mathcal{E}_{i}\right) \perp \mathcal{D}_{1}$ and $\left(\mathcal{E}_{i}\right) \perp \mathcal{D}_{2}$.

Proof.

(a) By construction, the root action $x$ is never repeated in $x^{+} \cdot\left\{\mathcal{E}_{1}, \ldots, \mathcal{E}_{n}\right\}$. By definition of VAM, the interaction in the cut-net $\left\{x^{+} .\left\{\mathcal{E}_{1}, \ldots, \mathcal{E}_{n}\right\}, x^{-} . \mathcal{D}\right\}$ starts by matching $x^{+}$ with $x^{-}$, and then continue as in $\left\{\mathcal{E}_{1}, \ldots, \mathcal{E}_{n}, \mathcal{D}\right\}$. More precisely,

$V A M\left(x^{+} .\left\{\mathcal{E}_{1}, \ldots, \mathcal{E}_{n}\right\}, x^{-} . \mathcal{D}\right)=\left\{x^{+}, x^{+} . x^{-}\right\} \cup\left\{x^{+} . x^{-} . q: q \in V A M\left(\mathcal{E}_{1}, \ldots, \mathcal{E}_{n}, \mathcal{D}\right)\right\}$.

If $I\left(\mathcal{E}_{1}, \ldots, \mathcal{E}_{n}, \mathcal{D}\right) \neq \emptyset$, then

$$
I\left(x^{+} .\left\{\mathcal{E}_{1}, \ldots, \mathcal{E}_{n}\right\}, x^{-} . \mathcal{D}\right)=\left\{x^{+}, x^{+} . x^{-}\right\} \cup\left\{x^{+} . x^{-} . q: q \in I\left(\mathcal{E}_{1}, \ldots, \mathcal{E}_{n}, \mathcal{D}\right)\right\} .
$$

If $I\left(\mathcal{E}_{1}, \ldots, \mathcal{E}_{n}, \mathcal{D}\right)=\emptyset$, then $I\left(x^{+} .\left\{\mathcal{E}_{1}, \ldots, \mathcal{E}_{n}\right\}, x^{-} . \mathcal{D}\right)$ is also empty. By hiding, we have the conclusion.

(b) By definition of VAM,

$$
\begin{aligned}
V A M\left(\mathcal{E}_{1}, \ldots, \mathcal{E}_{n}, \mathcal{D}_{1} \oplus^{\tau} \mathcal{D}_{2}\right) & =\left\{t . t_{1} . q: q \in V A M\left(\mathcal{E}_{1}, \ldots, \mathcal{E}_{n}, \mathcal{D}_{1}\right)\right\} \\
& \cup\left\{t . t_{2} . q: q \in \operatorname{VAM}\left(\mathcal{E}_{1}, \ldots, \mathcal{E}_{n}, \mathcal{D}_{2}\right)\right\} .
\end{aligned}
$$

Since

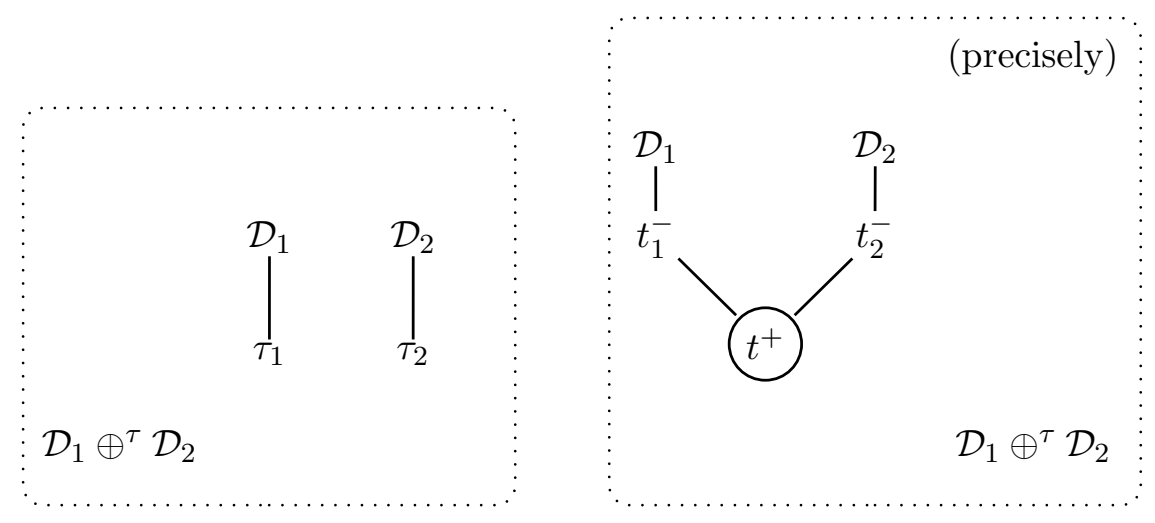

we have 


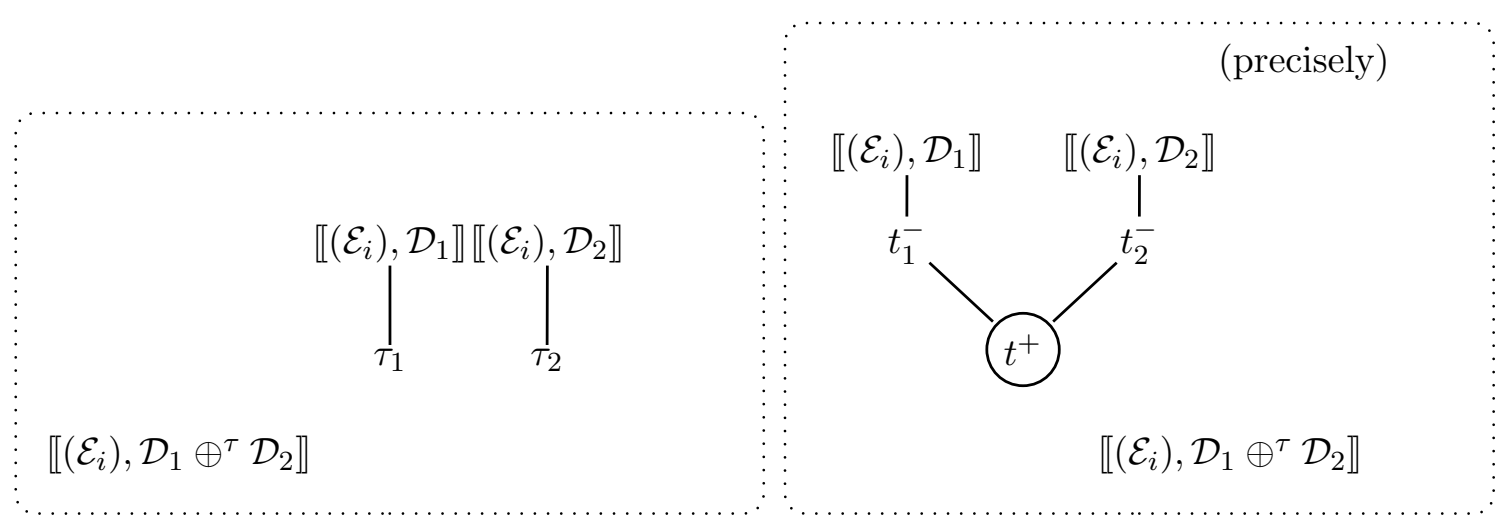

9.2. Interactive types. As already defined in Section 4.3, a behaviour $\mathbf{G}$ is a set of strategies closed by biorthogonal. Since we have abandoned the limitation of "strict linearity" which we had in Section 4.1.2, now behaviours are never empty.

Definition 9.5. A behaviour on the interface $\Gamma$ is a set $\mathbf{G}$ of strategies $\mathcal{D}: \Gamma$ such that $\mathbf{G}^{\perp \perp}=\mathbf{G}$. A behaviour is positive or negative according to the polarity of the interface.

Proposition 9.6. A behaviour is always non-empty.

Proof. A positive behaviour always contains at least $\mathfrak{D a i}$. A negative behaviour on the interface $\sigma^{-}, \xi_{1}^{+}, \ldots, \xi_{n}^{+}$, always contains at least the following strategy $\mathfrak{D a i}{ }^{-}$(called negative daimon in [22]):

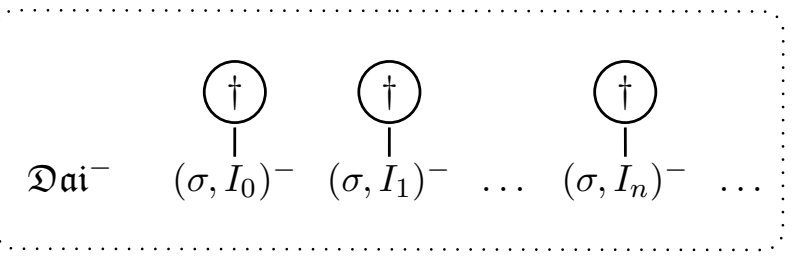

Indeed, let $\mathcal{D}: \sigma^{+}$and $\mathcal{E}_{1}: \xi_{1}^{-}, \ldots, \mathcal{E}_{n}: \xi_{n}^{-}$be total counter-strategies. Let us consider then the closed cut-net $\left\{\mathfrak{D a i} \mathfrak{a}^{-}, \mathcal{D}, \mathcal{E}_{1}, \ldots, \mathcal{E}_{n}\right\}$ and calculate its normal form. By definition of the abstract machine, we start by collecting actions in $\mathcal{D}$ (because it is the main strategy). Since $\mathcal{D}$ is total, after a sequence of silent actions we reach $\dagger$ or we reach a positive action $\left(\sigma, I_{n}\right)^{+}$ (both cases are possible "in parallel", since $\mathcal{D}$ is non-uniform). In the latter case this proper action matches its opposite $\left(\sigma, I_{n}\right)^{-}$and we eventually reach $\dagger$. The normal form is then a total strategy on the empty interface, since each path from the root of $\llbracket \mathfrak{D a i}{ }^{-}, \mathcal{D}, \mathcal{E}_{1}, \ldots, \mathcal{E}_{n} \rrbracket$ leads to $\dagger$. 
Example $9.7\left(?_{P} \mathbf{0}, !_{N} \mathrm{~T}\right)$. Let us see what happens to the behaviour generated from $\mathfrak{D a i}: \xi^{+}(c f$. Example 4.33) in the non-linear setting.

$$
?_{P} \mathbf{0}:=\{\mathfrak{D a i}\}^{\perp \perp} \text { on interface } \xi^{+} ; \quad !_{N} \top:=\{\mathfrak{D a i}\}^{\perp} \text { on interface } \xi^{-} .
$$

We have that $?_{P} \mathbf{0}$ contains a unique deterministic strategy, which is $\mathfrak{D} \mathfrak{a}$. $?_{P} \mathbf{0}$ also contains strategies which are set of views of the form $s=t . t_{i} \ldots . t . t_{j} \cdot \dagger$. On the other side, $!_{N} \top$ contains all negative strategies which have interface $\xi$, including the empty one.

When we take $?_{P} \mathbf{0}$ on the empty interface, it consists exactly of all the total strategies on the empty interface.

\section{LudiCs With REPETITIONS: TYPES AND INTERNAL COMPLETENESS}

In this section, we give constructions for the behaviours which correspond to the construction of MELLS formulas, and prove that they enjoy internal completeness.

10.1. MELLS types. In this section, we use the same constructions on strategies as in Section 4.5. The resulting behaviours are different, because normalization is different (i.e., non-linear).

10.1.1. Constructions on strategies. Let $\mathcal{D}_{i}: \xi i^{-}, \Pi(1 \leq i \leq n)$ be $n \geq 0$ negative strategies (which can possibly also be empty). We obtain a new positive strategy on the interface $\xi^{+}, \Pi$, denoted by $\mathcal{D}_{1} \bullet \cdots \bullet \mathcal{D}_{n}$, by adding to the union of the strategies the positive root $\left(\xi, I_{n}\right)^{+}$, i.e.,

$$
\mathcal{D}_{1} \bullet \cdots \bullet \mathcal{D}_{n}:=\left(\xi, I_{n}\right)^{+} .\left\{\mathcal{D}_{1}, \ldots, \mathcal{D}_{n}\right\}
$$

We observe that the root of the resulting strategy is linear. A converse construction also exists.

Lemma 10.1. Let $\mathcal{D}: \xi^{+}, \Pi$ be a positive strategy having as root a linear occurrence of the proper action $\left(\xi, I_{n}\right)^{+}$. We can write $\mathcal{D}$ as $\mathcal{D}_{1} \bullet \cdots \bullet \mathcal{D}_{n}$, with $\mathcal{D}_{i}: \xi i^{-}, \Pi$ for each $1 \leq i \leq n$.

Proof. Let us write $\mathcal{D}$ as $\left(\xi, I_{n}\right)^{+}$. $\mathcal{E}$. All views in $\mathcal{E}$ have a first action of the form $(\xi i, K)^{-}$, with $1 \leq i \leq n$. We partition $\mathcal{E}$ into maximal subsets of views which start with an action on the same name, that is, we set $\mathcal{D}_{i}:=\left\{s \in \mathcal{E}: s=(\xi i, K)^{-} . s^{\prime}\right.$ for some $\left.K\right\}$. Then it is immediate to verify that each $\mathcal{D}_{i}$ is in fact a negative strategy on interface $\xi i^{-}, \Pi$ and that $\mathcal{E}=\mathcal{D}_{1} \cup \ldots \cup \mathcal{D}_{n}$. We finally conclude $\mathcal{D}=\mathcal{D}_{1} \bullet \cdots \bullet \mathcal{D}_{n}$.

Lemma 10.2. Let $\mathcal{D}_{1}: \xi 1^{-}, \Pi, \ldots, \mathcal{D}_{n}: \xi n^{-}, \Pi$ be negative strategies. Let $\left(\mathcal{F}_{\alpha}\right)_{\alpha \in \Pi}$ be a family of counter-strategies (w.r.t. П). We have that

$$
\llbracket \mathcal{D}_{1} \bullet \cdots \bullet \mathcal{D}_{n},\left(\mathcal{F}_{\alpha}\right) \rrbracket=\llbracket \mathcal{D}_{1},\left(\mathcal{F}_{\alpha}\right) \rrbracket \bullet \cdots \bullet \llbracket \mathcal{D}_{n},\left(\mathcal{F}_{\alpha}\right) \rrbracket .
$$

That is,

$$
\llbracket\left(\xi, I_{n}\right)^{+} \cdot\left\{\mathcal{D}_{1}, \ldots, \mathcal{D}_{n}\right\},\left(\mathcal{F}_{\alpha}\right) \rrbracket=\left(\xi, I_{n}\right)^{+} \cdot\left\{\llbracket \mathcal{D}_{1},\left(\mathcal{F}_{\alpha}\right) \rrbracket, \ldots, \llbracket \mathcal{D}_{n},\left(\mathcal{F}_{\alpha}\right) \rrbracket\right\} .
$$

Proof. It easily follows from the definition of VAM. We first observe that by construction, the root of $\mathcal{D}_{1} \bullet \cdots \bullet \mathcal{D}_{n}$ is $\left(\xi, I_{n}\right)^{+}$and that it is a visible action. This implies that it is also the root of $\llbracket \mathcal{D}_{1} \bullet \cdots \bullet \mathcal{D}_{n},\left(\mathcal{F}_{\alpha}\right) \rrbracket$. Since $\left(\xi, I_{n}\right)^{+}$occurs linearly, the interaction never uses (occurrences of) $\left(\xi, I_{n}\right)^{+}$again, and we can write the equations above. 
From now until the end of Section 10, let us fix a family of $n(n \geq 0)$ negative behaviours $\mathbf{N}_{\xi i}: \xi_{i}^{-}$, with $1 \leq i \leq n$. We define

$$
\mathbf{N}_{\xi 1} \bullet \cdots \bullet \mathbf{N}_{\xi n}:=\left\{\mathcal{D}_{1} \bullet \cdots \bullet \mathcal{D}_{n}: \mathcal{D}_{i} \in \mathbf{N}_{\xi i}\right\}
$$

We define a new positive (resp. negative) behaviour on the interface $\xi^{+}$(resp. $\xi^{-}$) as follows:

$$
\mathbf{F}_{\xi}^{+}\left(\mathbf{N}_{\xi 1}, \ldots, \mathbf{N}_{\xi n}\right):=\left(\mathbf{N}_{\xi 1} \bullet \ldots \bullet \mathbf{N}_{\xi n}\right)^{\perp \perp} ; \quad \mathbf{F}_{\xi}^{-}\left(\mathbf{N}_{\xi 1}^{\perp}, \ldots, \mathbf{N}_{\xi n}^{\perp}\right):=\left(\mathbf{N}_{\xi 1} \bullet \cdots \bullet \mathbf{N}_{\xi n}\right)^{\perp}
$$

Remark 10.3. We stress once more that, by construction, all strategies in $\mathbf{N}_{\xi 1} \bullet \cdots \bullet \mathbf{N}_{\xi n}$ have as root $x=\left(\xi, I_{n}\right)^{+}$, which is linear. The repetitions of occurrences of $x$ are obtained via the closure by biorthogonality, and hence only belong to $\left(\mathbf{N}_{\xi 1} \bullet \cdots \bullet \mathbf{N}_{\xi n}\right)^{\perp \perp}$.

When $n=0$ we write $?_{P} \mathbf{1}$ and $!_{N} \perp$ for the positive behaviour and the negative one given by the previous constructions respectively. Precisely, ? $1=\{\mathcal{D}\}^{\perp \perp}$ and $!_{N} \perp=\{\mathcal{D}\}^{\perp}$, where $\mathcal{D}=(\xi, \emptyset)^{+}$.

Lemma 10.4. If the root $x$ of $\mathcal{D} \in \mathbf{P}_{\xi}=\left(\mathbf{N}_{\xi 1} \bullet \cdots \bullet \mathbf{N}_{\xi n}\right)^{\perp \perp}$ is a proper action, then $x=\left(\xi, I_{n}\right)^{+}$.

Proof. By construction, all the strategies in $\mathbf{N}_{\xi 1} \bullet \cdots \bullet \mathbf{N}_{\xi n}$ have root $x=\left(\xi, I_{n}\right)^{+}$. Hence, $\left(\mathbf{N}_{\xi 1} \bullet \cdots \bullet \mathbf{N}_{\xi n}\right)^{\perp}$ contains the strategy $\mathcal{E}:=\left(\xi, I_{n}\right)^{-} . \dagger$. Since any $\mathcal{D} \in\left(\mathbf{N}_{\xi 1} \bullet \cdots \bullet \mathbf{N}_{\xi n}\right)^{\perp \perp}$ has to be orthogonal to $\mathcal{E}$, when its root is a proper action it must be $\left(\xi, I_{n}\right)^{+}$.

10.2. Sequent of behaviours. The definition of sequent of behaviours remains the same as in the linear case, and Proposition 4.39] still holds. For the reader's convenience we repeat them below.

Definition 10.5 (Sequent of behaviours). Let $\Gamma=\xi_{1}^{\epsilon_{1}}, \ldots, \xi_{n}^{\epsilon_{n}}(n \geq 0)$ be an interface, and let $\boldsymbol{\Gamma}=\mathbf{G}_{\xi_{1}}, \ldots, \mathbf{G}_{\xi_{n}}(n \geq 0)$ behaviours of respective polarities $\epsilon_{1}, \ldots, \epsilon_{n}$.

We define a new behaviour on the same interface $\Gamma$, which we call sequent of behaviours and denote by $\vdash \boldsymbol{\Gamma}$, as follows:

$\vdash \boldsymbol{\Gamma}:=\left\{\mathcal{D}: \mathcal{D}\right.$ is total on interface $\Gamma$ and $\mathcal{D} \perp\left\{\mathcal{E}_{1}, \ldots, \mathcal{E}_{n}\right\}$ for all $\left.\mathcal{E}_{1} \in \mathbf{G}_{\xi_{1}}^{\perp}, \ldots, \mathcal{E}_{n} \in \mathbf{G}_{\xi_{n}}^{\perp}\right\}$.

Observe that if $\Gamma$ is empty, then $\vdash \Gamma$ consists of those strategies on the empty interface which are total (cf. Section 7). Hence, $\vdash \boldsymbol{\Gamma}$ is $\{\mathfrak{D a} \mathfrak{a}\}^{\perp \perp}$.

We will use the following two results.

Proposition 10.6. Let $\mathbf{A}, \mathbf{G}_{1}, \ldots \mathbf{G}_{n}(n \geq 0)$ be a sequence of behaviours, and $\boldsymbol{\Gamma}=$ $\mathbf{G}_{1}, \ldots \mathbf{G}_{n}$. We have that:

- $\mathcal{D} \in \vdash \boldsymbol{\Gamma}, \mathbf{A}$ if and only if for each $\mathcal{F} \in \mathbf{A}^{\perp}, \llbracket \mathcal{D}, \mathcal{F} \rrbracket \in \vdash \boldsymbol{\Gamma}$.

- $\mathcal{D} \in \vdash \mathbf{\Gamma}, \mathbf{A}$ if and only if $\llbracket \mathcal{D},\left(\mathcal{E}_{i}\right) \rrbracket \in \vdash \mathbf{A}$, for each family $\left(\mathcal{E}_{i}\right)$ such that $\mathcal{E}_{1} \in \mathbf{G}_{1}^{\perp}, \ldots$, $\mathcal{E}_{n} \in \mathbf{G}_{n}^{\perp}$.

The proof is the same as for Proposition 4.39 (but now using Theorem 8.13). Observe again that if $\Gamma=\emptyset$, the condition in the first claim is simply a reformulation of the definition of orthogonality: $\mathcal{D} \perp \mathcal{F}$ if $\llbracket \mathcal{D}, \mathcal{F} \rrbracket$ is a total strategy on the empty interface. 
Lemma 10.7. Let $\mathbf{N}=\left(\mathbf{N}_{\xi 1} \bullet \cdots \bullet \mathbf{N}_{\xi n}\right)^{\perp}$. We have that

(1) If $\mathcal{F} \in \mathbf{N}$, then $\mathcal{F} \neq \emptyset$.

(2) If $\mathcal{F} \in \vdash \mathbf{N}, \boldsymbol{\Pi}$, then $\mathcal{F} \neq \emptyset$.

Proof.

(1) $\mathcal{F} \neq \emptyset$ because each $\mathcal{D} \in \mathbf{N}_{\xi 1} \bullet \cdots \bullet \mathbf{N}_{\xi n}$ has root $\left(\xi, I_{n}\right)^{+}$, and $\mathcal{F}$ has to be orthogonal to $\mathcal{D}$.

(2) The second point is proven with a similar argument.

10.3. Internal completeness. The following two propositions are the core of internal completeness. Internal completeness for a negative behaviour is the same as in the linear case. For the positive case, we also first state internal completeness for those strategies whose root is linear. The key element which allows us to reduce the general case to this one is Lemma 10.10.

Proposition 10.8 (Internal completeness of $\mathbf{F}^{-}$). Let $\mathbf{P}_{\xi i}=\mathbf{N}_{\xi i}^{\perp}$ and $x=\left(\xi, I_{n}\right)$.

$$
\text { (1) } x^{-} . \mathcal{D} \in \mathbf{F}^{-}\left(\mathbf{P}_{\xi_{1}}, \ldots, \mathbf{P}_{\xi_{n}}\right) \Leftrightarrow(2) \mathcal{D} \in \vdash \mathbf{P}_{\xi_{1}}, \ldots, \mathbf{P}_{\xi_{n}} .
$$

Proof. The proof follows immediately from the definitions. Expanding the definition, we obtain the two following properties, which are equivalent by using Lemma 9.4 (a):

(1) $x^{-} . \mathcal{D} \perp x^{+} .\left\{\mathcal{E}_{1}, \ldots, \mathcal{E}_{n}\right\}$, for any $\mathcal{E}_{1} \in \mathbf{P}_{\xi_{1}}^{\perp}, \ldots, \mathcal{E}_{n} \in \mathbf{P}_{\xi_{n}}^{\perp}$;

(2) $\mathcal{D} \perp\left\{\mathcal{E}_{1}, \ldots, \mathcal{E}_{n}\right\}$, for any $\mathcal{E}_{1} \in \mathbf{P}_{\xi_{1}}^{\perp}, \ldots, \mathcal{E}_{n} \in \mathbf{P}_{\xi_{n}}^{\perp}$.

Proposition 10.9 (Linear internal completeness of $\left.\mathbf{F}^{+}\right)$. Let $\mathcal{D} \in \mathbf{F}^{+}\left(\mathbf{N}_{\sigma 1}, \ldots, \mathbf{N}_{\sigma n}\right)$ have a root that is a linear occurrence of a proper action. Then $\mathcal{D}=\mathcal{D}_{1} \bullet \cdots \bullet \mathcal{D}_{n}$, with $\mathcal{D}_{i} \in \mathbf{N}_{\sigma i}$, for any $1 \leq i \leq n$.

Proof. By Lemma 10.4 we know that the root of $\mathcal{D}$ must be $\left(\sigma, I_{n}\right)^{+}$. Since it occurs linearly, we can apply Lemma 10.1 and then write $\mathcal{D}$ as $\mathcal{D}_{1} \bullet \cdots \bullet \mathcal{D}_{n}$. We already observed (Remark 5.2), that the argument in Proposition 5.1 only relies on the fact that the root is a linear occurrence of action. We can hence repeat the same proof as in 5.1.

The essential property which allows us to use the argument we sketched in Section 6.4 is the following Lemma 10.10 (1). Point (2) guarantees that all tests we are interested in have the form requested by point (1).

Lemma 10.10. Let $\mathbf{N}_{\xi}=\left(\mathbf{N}_{\xi 1} \bullet \cdots \bullet \mathbf{N}_{\xi n}\right)^{\perp}, \mathbf{P}_{\xi}=\left(\mathbf{N}_{\xi 1} \bullet \cdots \bullet \mathbf{N}_{\xi n}\right)^{\perp \perp}$, and $x=\left(\xi, I_{n}\right)$.

(1) Let $\mathcal{F}_{1}, \mathcal{F}_{2} \in \mathbf{N}_{\xi}$. Assume $\mathcal{F}_{1}=x^{-} . \mathcal{D}_{1}, \mathcal{F}_{2}=x^{-} . \mathcal{D}_{2}$. We have that $\mathcal{F}_{1}+{ }^{\tau} \mathcal{F}_{2} \in \mathbf{N}_{\xi}$ (cf. Definition 7.5).

(2) Let us denote by $\mathbf{N}_{\xi}\langle x\rangle$ the set of all $\mathcal{F} \in \mathbf{N}_{\xi}$ such that $\mathcal{F}$ has $x$ as unique root. We have that $\left(\mathbf{N}_{\xi}\langle x\rangle\right)^{\perp}=\mathbf{N}_{\xi}^{\perp}$ i.e.,

$$
\mathbf{P}_{\xi}=\left(\mathbf{N}_{\xi}\langle x\rangle\right)^{\perp}
$$

Proof.

(1) By internal completeness (Proposition 10.8), $\mathcal{D}_{1}, \mathcal{D}_{2} \in \vdash \mathbf{N}_{\xi 1}^{\perp}, \ldots, \mathbf{N}_{\xi n}^{\perp}$. By Lemma 9.4, $\mathcal{D}_{1} \oplus^{\tau} \mathcal{D}_{2} \in \vdash \mathbf{N}_{\xi 1}^{\perp}, \ldots, \mathbf{N}_{\xi n}^{\perp}$. By Proposition 10.8 again, we conclude $\mathcal{F}_{1}+\tau \mathcal{F}_{2}=$ $x^{-} .\left(\mathcal{D}_{1} \oplus^{\tau} \mathcal{D}_{2}\right) \in \mathbf{N}$. 
(2) Let $\mathcal{F} \in\left(\mathbf{N}_{\xi 1} \bullet \cdots \bullet \mathbf{N}_{\xi n}\right)^{\perp}$. Being on interface $\xi$, a negative strategy $\mathcal{F}$ may have several roots, all of the form $(\xi, K)^{-}$(all views in $\mathcal{F}$ start with such an action). We define $\mathcal{F}_{x} \subseteq \mathcal{F}$ as the subset of views whose first action is $x=\left(\xi, I_{n}\right)$. $\mathcal{F}_{x}$ is clearly a strategy. We proceed in two steps.

(i) We prove that for each $\mathcal{F} \in \mathbf{N}_{\xi}$, then $\mathcal{F}_{x} \in \mathbf{N}_{\xi}$.

Let $\mathcal{D}: \xi^{+} \in \mathbf{N}_{\xi 1} \bullet \cdots \bullet \mathbf{N}_{\xi n}$. A positive strategy has a unique root. By construction, the root of $\mathcal{D}$ is the action $x^{+}=\left(\xi, I_{n}\right)^{+}$. For each $\mathcal{F} \in \mathbf{N}_{\xi}$, it must be $\mathcal{D} \perp \mathcal{F}$. Since $\mathcal{D}$ is the main strategy in the cut-net $\{\mathcal{D}, \mathcal{F}\}, \mathcal{F}$ need to have $x^{-}=\left(\xi, I_{n}\right)^{-}$as a root, in order to match $x^{+}$, otherwise those strategies would not be orthogonal. We observe that the normalization with any $\mathcal{D} \in \mathbf{N}_{\xi 1} \bullet \cdots \bullet \mathbf{N}_{\xi n}$ only uses views in $\mathcal{F}_{x}$, because $\mathcal{D}$ by construction does not contain any other occurrence of action on the name $\xi$ but the root. As a consequence, $\llbracket \mathcal{D}, \mathcal{F} \rrbracket=\llbracket \mathcal{D}, \mathcal{F}_{x} \rrbracket$.

(ii) We can now prove that $\left(\mathbf{N}_{\xi}\langle x\rangle\right)^{\perp} \subseteq \mathbf{N}_{\xi}^{\perp}$ (the other inclusion is obvious, since $\left.\mathbf{N}_{\xi}\langle x\rangle \subseteq \mathbf{N}_{\xi}\right)$. Let $\mathcal{D} \in\left(\mathbf{N}_{\xi}\langle x\rangle\right)^{\perp}$. We prove that for each $\mathcal{F} \in \mathbf{N}_{\xi}, \mathcal{D} \perp \mathcal{F}$, i.e., $\mathcal{D} \in \mathbf{N}_{\xi}^{\perp}$. More precisely, we prove that $\llbracket \mathcal{D}, \mathcal{F} \rrbracket=\llbracket \mathcal{D}, \mathcal{F}_{x} \rrbracket$, by showing that the part of $\mathcal{F}$ used in the interaction against $\mathcal{D}$ is all contained in $\mathcal{F}_{x}$.

Assume there exists $p \in I(\mathcal{D}, \mathcal{F})$ such that $\ulcorner p\urcorner \notin \mathcal{F}_{x}$, and let us take it minimal, i.e., $\ulcorner p\urcorner=y^{-}$, where $y=(\xi, J), J \neq I_{n}$ (i.e., the interaction enters the root of another subtree of $\mathcal{F}_{y}$ of $\left.\mathcal{F}\right)$. In this case $p=p^{\prime} . y^{+} . y^{-}$, with $\ulcorner t\urcorner \in\left\{\mathcal{D}, \mathcal{F}_{x}\right\}$, for each $t \sqsubseteq p^{\prime} . y^{+}$. We observe that $p^{\prime} . y^{+}$has no extensions in $\operatorname{VAM}\left(\mathcal{D}, \mathcal{F}_{x}\right)$ (because there is no match for $y^{+}$), and that it contains no $\dagger$. Let $s$ be the maximal prefix of $p^{\prime} . y^{+}$which has an extension $q . \dagger$ in $\operatorname{VAM}\left(\mathcal{D}, \mathcal{F}_{x}\right)$ (i.e., an extension which terminates with a $\dagger$ action). We have $s . a \sqsubseteq p^{\prime} . y^{+}, s . b \sqsubseteq q . \dagger$, and by Lemma 8.10, we have that $a, b$ are negative silent actions. Hence s.a $\in I\left(\mathcal{D}, \mathcal{F}_{x}\right)$, and hide $($ s.a $) \in \llbracket \mathcal{D}, \mathcal{F}_{x} \rrbracket$, but hide $(s . a)$ has no extension terminating with a $\dagger$ action, against the hypothesis that $\mathcal{D} \perp \mathcal{F}_{x}$.

Observe that, putting all elements together, we have also established that

$$
\llbracket \mathcal{D}, \mathcal{F} \rrbracket=\llbracket \mathcal{D}, \mathcal{F}_{x} \rrbracket \text { for each } \mathcal{D} \in \mathbf{N}_{\xi}^{\perp}, \mathcal{F} \in \mathbf{N}_{\xi} .
$$

We will use this in the sequel.

Observe that the property at the point (1) above does not hold in general, for arbitrary behaviours (see Example 9.3 and take $\mathbf{G}=\{\mathcal{D}\}^{\perp}$ ).

Lemma 10.11. Let $\mathcal{D} \in \mathbf{P}_{\xi}=\left(\mathbf{N}_{\xi 1} \bullet \cdots \bullet \mathbf{N}_{\xi n}\right)^{\perp \perp}$, such that the root is a proper action. We have that $\underline{\sigma}(\mathcal{D}) \in \vdash \mathbf{P}_{\xi}, \mathbf{P}_{\sigma}$. Moreover, the new root on $\sigma$ is linear.

Proof. By Lemma $10.10(2), \mathbf{P}_{\xi}=\left(\mathbf{N}_{\xi}\langle x\rangle\right)^{\perp}$. For all pairs $\mathcal{E}, \mathcal{F} \in \mathbf{N}_{\xi}\langle x\rangle$, we have $\mathcal{D} \perp \mathcal{E}$ and $\mathcal{D} \perp \mathcal{F}$. By Lemma 10.10 (1), we have that $\mathcal{D} \perp\left(\mathcal{E}+{ }^{\tau} \mathcal{F}\right)$. Using Proposition 8.15, we have that $\underline{\sigma}(\mathcal{D}) \perp\left\{\mathcal{E}+^{\tau} \mathcal{F},\left(\mathcal{E}+{ }^{\tau} \mathcal{F}\right)[\sigma / \xi]\right\}$ and by using twice Lemma 9.2 we have that $\underline{\sigma}(\mathcal{D}) \perp\{\mathcal{E}, \mathcal{F}[\sigma / \xi]\}$, that is $\underline{\sigma}(\mathcal{D}) \in \vdash \mathbf{P}_{\xi}, \mathbf{P}_{\sigma}$. The linearity of $\underline{\sigma}(\mathcal{D})$ is given by the construction " $\underline{\sigma}(-)$.

Corollary 10.12 (Internal completeness of $\left.\mathbf{F}^{+}\right)$. Let $\mathcal{D} \in \mathbf{P}_{\xi}=\mathbf{F}^{+}\left(\mathbf{N}_{\xi 1}, \ldots, \mathbf{N}_{\xi n}\right)$ be as in Lemma 10.11. Then $\underline{\sigma}(\mathcal{D})=\mathcal{D}_{1}^{\prime} \bullet \cdots \bullet \mathcal{D}_{n}^{\prime}$ where each $\mathcal{D}_{i}^{\prime} \in \vdash \mathbf{N}_{\sigma i}, \mathbf{P}_{\xi}$.

Proof. By Lemma 10.4, the root $x$ of $\mathcal{D} \in \mathbf{P}_{\xi}$ is $x=\left(\xi, I_{n}\right)$. By Lemma 10.11, we have that $\underline{\sigma}(\mathcal{D}) \in \vdash \mathbf{P}_{\xi}, \mathbf{P}_{\sigma}$. The root $\left(\sigma, I_{n}\right)$ is the only occurrence of action on $\sigma$. By Lemma 10.1, we can write $\underline{\sigma}(\mathcal{D})$ as $\underline{\sigma}(\mathcal{D})=\mathcal{E}_{1} \bullet \cdots \bullet \mathcal{E}_{n}$. Now we use Proposition 10.6. For each $\mathcal{A}_{\xi} \in \mathbf{P}_{\xi}^{\perp}$, we have $\llbracket \underline{\sigma}(\mathcal{D}), \mathcal{A} \rrbracket \in \mathbf{P}_{\sigma}$. By Lemma 10.2 we have that: $\llbracket \mathcal{E}_{1} \bullet \cdots \bullet \mathcal{E}_{n}, \mathcal{A} \rrbracket=\llbracket \mathcal{E}_{1}, \mathcal{A} \rrbracket \bullet \cdots \bullet \llbracket \mathcal{E}_{1}, \mathcal{A} \rrbracket$. 
By Proposition 10.9, $\llbracket \mathcal{E}_{i}, \mathcal{A} \rrbracket \in \mathbf{N}_{\sigma i}$. By using again Proposition 10.6, we have that $\mathcal{E}_{i} \in \vdash$ $\mathbf{N}_{\sigma i}, \mathbf{P}_{\xi}$.

\section{LUdics With REPETITIONS: FULL COMPLETENESS}

In this section, we show that our model is fully complete with respect to MELLS (Section 2).

As usual in game semantics (e.g., [31, 33]), not all strategies are suitable to be interpretation of a proof. In general, strategies which are interpretations of a proof have to satisfy some winning conditions which describe a strategy with "good properties." Our winning strategies are those that are finite, deterministic, daimon-free and material (see below).

We now introduce the notion of materiality.

11.1. Materiality. It is important to have in mind that normalization does not necessarily visit all the actions of a strategy. This is exactly what underlies the notion of materiality. Let us first examine an example, to understand materiality.

By definition of normalization (Section 8), at each step, the machine examines (i.e., visits) an occurrence of action $a$ in a view s.a belonging to a strategy of the cut-net. We say that the view s.a is used or visited.

Example 11.1. Let $\mathcal{D}, \mathcal{E}, \mathcal{F}$ be the strategies in Figure 7.

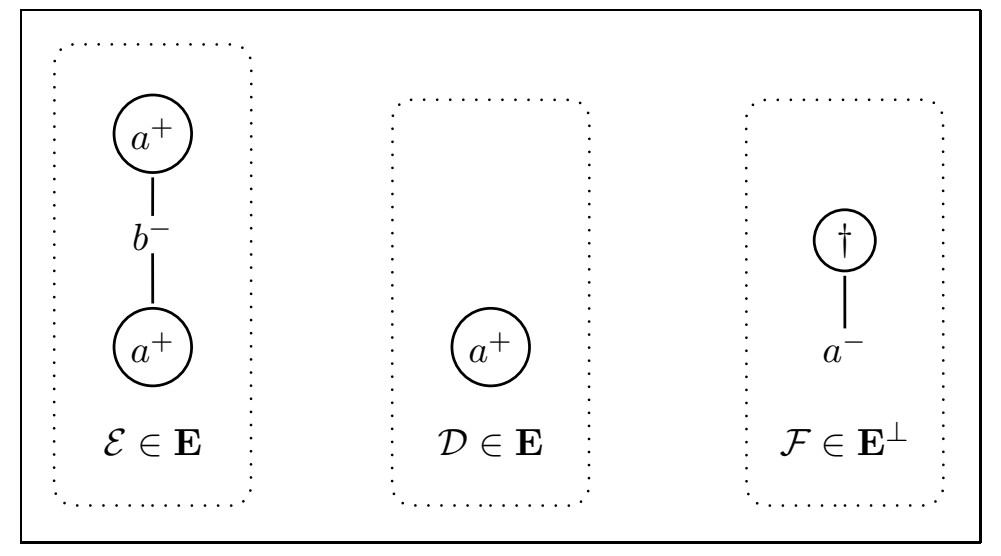

Figure 7: Materiality

Consider the behaviour $\mathbf{E}=\{\mathcal{D}\}^{\perp \perp}$ and notice that $\mathcal{F} \in \mathbf{E}^{\perp}$. Observe also that $\mathcal{E} \in \mathbf{E}$, but the normalization between $\mathcal{E}$ and $\mathcal{F}$ uses only the first action $a^{+}$; the action $b^{-}$is never visited through the interaction between $\mathcal{E}$ and $\mathcal{F}$.

The notion of materiality exactly captures the significant part of a strategy $\mathcal{D}$ in a behaviour, i.e., the part that is really used to react to the tests in $\mathbf{E}^{\perp}$.

To make these ideas formal, we proceed in two steps.

First, in Section 11.1.1, we consider a strategy $\mathcal{D}$, and a family of counter-strategies $\left(\mathcal{E}_{i}\right)$. We define the restriction of $\mathcal{D}$ to the part $\mathcal{D}^{\prime} \subseteq \mathcal{D}$ which is really used (visited) to produce the normal form $\llbracket \mathcal{D},\left(\mathcal{E}_{i}\right) \rrbracket$. We have that $\mathcal{D}^{\prime}$ is a strategy, and that $\llbracket \mathcal{D}^{\prime},\left(\mathcal{E}_{i}\right) \rrbracket=\llbracket \mathcal{D},\left(\mathcal{E}_{i}\right) \rrbracket$. Any 
occurrence of action in $\mathcal{D}$ which is outside $\mathcal{D}^{\prime}$ will never be accessed during this normalization and in fact those - not visited - actions are not significant in the calculation.

Then, in Section 11.1.2, we consider $\mathcal{D}$ inside a behaviour G. From the point of view of the behaviour $\mathbf{G}$, the part of $\mathcal{D}$ which is significant is the part that is really used to react to the tests (strategies of $\mathbf{G}^{\perp}$ ). This leads to the definition of material part of a strategy in a behaviour.

11.1.1. Part of a cut-net used by the normalization. When normalizing two strategies $\mathcal{D}, \mathcal{E}$, only a part $\mathcal{D}^{\prime} \subseteq \mathcal{D}, \mathcal{E}^{\prime} \subseteq \mathcal{E}$ is used to produce the normal form; this is the set of views which are examined by the machine in Definition 8.4. If we run the machine on $\{\mathcal{D}, \mathcal{E}\}$ or on $\left\{\mathcal{D}^{\prime}, \mathcal{E}^{\prime}\right\}$, the result will be the same.

Definition 11.2. Let $\mathcal{R}$ be a cut-net. For each $\mathcal{D} \in \mathcal{R}$, we define the restriction of $\mathcal{D}$ to those of its views which are used (or visited) in the process of interaction to produce $\llbracket \mathcal{R} \rrbracket$ as follows:

$$
\mathcal{D}^{\mathcal{R}}=\mathcal{D} \cap\{\ulcorner p\urcorner: p \in I(\mathcal{R})\} .
$$

If $\mathcal{R}=\left\{\mathcal{D}, \mathcal{E}_{1}, \ldots, \mathcal{E}_{n}\right\}$, we will also write $\mathcal{D}\left[\mathcal{E}_{1}, \ldots, \mathcal{E}_{n}\right]$ for $\mathcal{D}^{\mathcal{R}}$.

Lemma 11.3. Let $\mathcal{R}$ be a cut-net. For each $\mathcal{D} \in \mathcal{R}, \mathcal{D}^{\mathcal{R}}$ is a strategy.

Proof. Let $\mathcal{D} \in \mathcal{R}$. Since $\mathcal{D}^{\mathcal{R}} \subseteq \mathcal{D}$, to show that it is a strategy, we only need to show that it satisfies $\tau$-Positivity, in the sense of Definition [7.2. Let $s . a$ be maximal in $\mathcal{D}^{\mathcal{R}}$. By construction, s. $a=\ulcorner p\urcorner$, for some $p=x_{1} \ldots x_{n} \in I(\mathcal{R})$. Moreover, by definition of view extraction \ulcorner\urcorner (Definition 8.1) $x_{n}=a$. If $a$ is a proper negative action, by definition of $I(\mathcal{R}), p$ must have an extension. Let us consider a minimal one, $p . c^{+}$. It has to be that $\ulcorner p\urcorner . c^{+} \in \mathcal{D}$ (by Definition 8.4 of VAM). We have that $\left\ulcorner p . c^{+}\right\urcorner=\ulcorner p\urcorner . c^{+}=s a . c^{+} \in \mathcal{D}^{\mathcal{R}}$, against the hypothesis that $s . a$ is maximal.

The part of a strategy which we have defined in Definition 11.2 is the part of the cut-net in which normalization takes place.

Lemma 11.4. Let $\mathcal{R}=\left\{\mathcal{D}_{1}, \ldots, \mathcal{D}_{n}\right\}$ be a cut-net. We have that

$$
\llbracket \mathcal{D}_{1}, \ldots, \mathcal{D}_{n} \rrbracket=\llbracket \mathcal{D}_{1}^{\mathcal{R}}, \ldots, \mathcal{D}_{n}^{\mathcal{R}} \rrbracket .
$$

Proof. It is a straightforward consequence of Definition 8.4, since all the views of $\mathcal{D}_{1}, \ldots, \mathcal{D}_{n}$ which are used for the construction of $I(\mathcal{R})$ are contained in $\mathcal{D}_{1}^{\mathcal{R}}, \ldots, \mathcal{D}_{n}^{\mathcal{R}}$.

11.1.2. Material part of a strategy in a behaviour. If $\mathcal{D}$ is a strategy in a behaviour $\mathbf{G}$, the part of $\mathcal{D}$ which is used to interact with all the tests (i.e., the strategies in $\mathbf{G}^{\perp}$ ) is the only significant part of $\mathcal{D}$ from the point of view of the behaviour.

To make the notion of materiality easier to grasp, we first give he definition in the case of behaviours on unary interfaces, and then generalize it to sequents of behaviours.

Definition 11.5 (Materiality). Let $\mathbf{G}$ be a behaviour and $\mathcal{D}$ a strategy in $\mathbf{G}$. We define the material part of $\mathcal{D}$ in $\mathbf{G}$ as

$$
|\mathcal{D}|_{\mathbf{G}}:=\bigcup\left\{\mathcal{D}[\mathcal{E}]: \mathcal{E} \in \mathbf{G}^{\perp}\right\} .
$$

A strategy $\mathcal{D} \in \mathbf{G}$ is said to be material in $\mathbf{G}$ if $\mathcal{D}=|\mathcal{D}|_{\mathbf{G}}$. 
For $\boldsymbol{\Gamma}=\mathbf{G}_{1}, \ldots, \mathbf{G}_{n}$, let $\vdash \boldsymbol{\Gamma}$ be a sequent of behaviours, $\mathcal{D} \in \vdash \boldsymbol{\Gamma}$, and $\left(\mathcal{E}_{i}\right)$ a family of counter-strategies $\mathcal{E}_{1} \in \mathbf{G}_{1}^{\perp}, \ldots, \mathcal{E}_{n} \in \mathbf{G}_{n}^{\perp}$. The material part of $\mathcal{D}$ in $\vdash \boldsymbol{\Gamma}$ is defined as

$$
|\mathcal{D}|_{\vdash \Gamma \boldsymbol{\Gamma}}:=\bigcup\left\{\mathcal{D}\left[\left(\mathcal{E}_{i}\right)\right]: \mathcal{E}_{1} \in \mathbf{G}_{1}^{\perp}, \ldots, \mathcal{E}_{n} \in \mathbf{G}_{n}^{\perp}\right\} .
$$

A strategy $\mathcal{D} \in \vdash \Gamma$ is said material in $\vdash \Gamma$ if $\mathcal{D}=|\mathcal{D}|_{\vdash \Gamma}$.

As an immediate consequence of Lemma 11.3, we have that:

Lemma 11.6. $|\mathcal{D}|_{\mathbf{G}}$ and $|\mathcal{D}|_{\vdash \boldsymbol{\Gamma}}$ are strategies.

Example 11.7. Let us consider $\mathcal{D}$ and $\mathcal{E}$ as in Example 11.1, We see that $\mathcal{D}$ is material in $\mathbf{E}$ whereas $\mathcal{E}$ is not.

The content of the definition of materiality is made explicit by the properties below.

Proposition 11.8. Let $\mathbf{G}$ be a behaviour on a unary interface and $\mathcal{D} \in \mathbf{G}$. We have:

(1) $\llbracket|\mathcal{D}|_{\mathbf{G}}, \mathcal{E} \rrbracket=\llbracket \mathcal{D}, \mathcal{E} \rrbracket$, for each $\mathcal{E} \in \mathbf{G}^{\perp}$.

(2) $|\mathcal{D}|_{\mathbf{G}} \perp \mathcal{E}$, for each $\mathcal{E} \in \mathbf{G}^{\perp}$. In particular, $|\mathcal{D}|_{\mathbf{G}} \in \mathbf{G}$.

Proof. By Lemma 11.4, we have that $\llbracket \mathcal{D}[\mathcal{E}], \mathcal{E} \rrbracket=\llbracket \mathcal{D}, \mathcal{E} \rrbracket$. From this we have (1) which implies (2).

The previous proposition obviously extends to sequents of behaviours.

Lemma 11.9. Let $\mathbf{N}=\left(\mathbf{N}_{\xi 1} \bullet \cdots \bullet \mathbf{N}_{\xi n}\right)^{\perp}$ be a behaviour on interface $\xi^{-}$. We have that if $\mathcal{F}$ is material in $\mathbf{N}$, then it has a single root which is $x=\left(\xi, I_{n}\right)^{-}$.

In other words, with the notations introduced in Lemma 10.10, we have $|\mathcal{F}|_{\mathbf{N}} \in \mathbf{N}\langle x\rangle$, for each $\mathcal{F} \in \mathbf{N}$.

Proof. The proof is actually part of the proof of Lemma 10.10 (2), because we have proven that given $\mathcal{F} \in \mathbf{N}$, for each $\mathcal{D} \in \mathbf{N}^{\perp}$, we have $\llbracket \mathcal{D}, \mathcal{F} \rrbracket=\llbracket \mathcal{D}, \mathcal{F}_{x} \rrbracket$, which in turn implies $\mathcal{F}[\mathcal{D}] \subseteq \mathcal{F}_{x}$.

Example 11.10 (Materiality with constant types). Let us fix a name $\xi$. The material and deterministic strategies in the constant type $?_{P} \mathbf{1}$ are $\mathcal{D}=(\xi, \emptyset)^{+}$and $\mathfrak{D} \mathfrak{a i}$. Moreover, $\mathcal{D}$ is winning in $?_{P} \mathbf{1}$ (see Definition 11.11).

The only material and deterministic strategy which inhabits $!_{N} \perp$ is $\mathcal{E}=(\xi, \emptyset)^{-} \cdot \mathfrak{D a i}$. In particular, there are no winning strategies in $!_{N} \perp$, i.e., " no syntactical derivations of $\vdash !_{N} \perp . "$

11.2. Completeness theorems. In Section 11.3, we describe the interpretation of a formula $F$ of MELLS into a behaviour $\mathbf{F}$ and similarly the interpretation of a syntactical sequent of MELLS into a sequent of behaviours. A derivation of a sequent in MELLS will be interpreted by a winning strategy which belongs to the interpretation of the sequent.

Definition 11.11 (Winning strategy). A strategy $\mathcal{D} \in \vdash \Gamma$ is said winning if it is finite, deterministic, daimon-free and material in $\vdash \boldsymbol{\Gamma}$.

In the sequel, finiteness, determinism, daimon-freeness and materiality are also called winning conditions. 
Remark 11.12 (Finiteness condition). We here assume finiteness among the winning conditions. However, recent work by Basaldella and Terui [6] shows an exciting property of interactive types: any material, deterministic and daimon free strategy in a behaviour which is interpretation of a logical formula is finite. We are confident that this result is also valid our setting; we need to check this in detail and we postpone it to a subsequent work.

The rest of this article is then devoted to proving the following theorems.

Soundness: (Theorem 11.16) Let $\pi$ be a derivation of a sequent $\vdash \Gamma$ in MELLS interpreted by a sequent of behaviours $\vdash \boldsymbol{\Gamma}$. There exists a winning strategy $\mathcal{D}(\pi) \in \vdash \boldsymbol{\Gamma}$.

Full Completeness: (Theorem 11.17) Let $\vdash \boldsymbol{\Gamma}$ be the interpretation of a sequent $\vdash \Gamma$ of MELLS and let $\mathcal{D} \in \vdash \boldsymbol{\Gamma}$. If $\mathcal{D}$ is winning, then $\mathcal{D}$ is the interpretation of a cut-free derivation $\pi$ of the sequent $\vdash \Gamma$ in MELLS.

11.3. Interpretation of formulas, sequents, derivations. In the rest of this work, we only consider behaviours inductively defined as follows, using the types constructors introduced in Section 10 .

$$
\mathbf{P}_{\xi}::=\mathbf{F}^{+}\left(\mathbf{N}_{\xi 1}, \ldots, \mathbf{N}_{\xi n}\right) \quad \mathbf{N}_{\xi}::=\mathbf{F}^{-}\left(\mathbf{P}_{\xi 1}, \ldots, \mathbf{P}_{\xi n}\right)
$$

where $n \geq 0$ and $\xi$ is an arbitrary name.

We now interpret formulas and sequents of MELLS. Intuitively, given a MELLS formula $F$ we associate to it a behaviour $\mathbf{F}$ of the same polarity. Given a sequent $\vdash$ $F_{1}, \ldots, F_{n}$, we associate to the occurrences of formula $F_{1}, \ldots, F_{n}$ behaviours $\mathbf{F}_{1}, \ldots, \mathbf{F}_{n}$ to form a sequent of behaviours $\vdash \mathbf{F}_{1}, \ldots, \mathbf{F}_{n}$.

Definition 11.13 (Interpretation of formulas and sequents of MELLS).

(1) Given a formula $F$ and an arbitrary name $\xi$ we associate a behaviour of the same polarity $\mathbf{F}$ on interface $\xi$ inductively as follows.

$$
\begin{gathered}
?_{P}\left(N_{1} \otimes \cdots \otimes N_{n}\right)^{\xi}:=\mathbf{F}^{+}\left(N_{1} \xi 1, \ldots, N_{n} \xi n\right) ; \\
!_{N}\left(P_{1} \not \ldots \otimes P_{n}\right)^{\xi}:=\mathbf{F}^{-}\left(P_{1} \xi 1, \ldots, P_{n} \xi n\right) .
\end{gathered}
$$

(2a) Given a positive sequent $\vdash P_{1}, \ldots, P_{n}$, and a positive interface $\xi_{1}^{+} \ldots, \xi_{n}^{+}$we associate the sequent of behaviours $\vdash P_{1} \xi_{1}, \ldots, P_{n} \xi_{n}$.

(2b) Given a negative sequent $\vdash N, P_{1}, \ldots, P_{n}$ and a negative interface $\sigma^{-}, \xi_{1}^{+} \ldots, \xi_{n}^{+}$we associate the sequent of behaviours $\vdash N^{\sigma}, P_{1} \xi_{1}, \ldots, P_{n} \xi_{n}$.

For instance, we have $?_{P} \mid \xi=?_{P} 1$ and $!_{N} \perp \xi=!_{N} \perp$ on interface $\xi^{+}$and $\xi^{-}$respectively.

In the sequel, we indicate the behaviour $F^{\xi}$ by $\mathbf{F}_{\xi}$ or just by $\mathbf{F}$ when the name $\xi$ is clear from the context or irrelevant for our purposes. Similarly, we write $\vdash \mathbf{P}_{\xi_{1}}, \ldots, \mathbf{P}_{\xi_{n}}$ and $\vdash \mathbf{N}_{\sigma}, \mathbf{P}_{\xi_{1}}, \ldots, \mathbf{P}_{\xi_{n}}$ or just $\vdash \mathbf{\Pi}$ and $\vdash \mathbf{N}, \boldsymbol{\Pi}$.

We are now ready to define the interpretation of a derivation $\pi$ of a sequent $\vdash \Gamma$ in MELLS.

Definition 11.14 (Interpretation of derivations of MELLS). Let $\pi$ be a derivation of a sequent $\vdash F_{1}, \ldots, F_{n}$ in MELLS and $\vdash \mathbf{F}_{\xi_{1}}, \ldots, \mathbf{F}_{\xi_{n}}$ its interpretation on an arbitrary interface $\xi_{1}, \ldots, \xi_{n}$. The interpretation of $\pi$ in $\vdash \mathbf{F}_{\xi_{1}}, \ldots, \mathbf{F}_{\xi_{n}}$ is a strategy $\mathcal{D}(\pi)$ on interface $\xi_{1}, \ldots, \xi_{n}$ inductively defined as follows.

Positive rule: $\pi$ ends with a positive rule $\operatorname{Pos}_{n}$ and the principal formula is $P=?_{P}\left(N_{1} \otimes\right.$ $\left.\cdots \otimes N_{n}\right)$. 


\begin{tabular}{ccc}
$\vdots \pi_{1}$ & & $\vdots \pi_{n}$ \\
$\vdash \Pi, P, N_{1}$ & $\ldots$ & $\vdash \Pi, P, N_{n}$ \\
\hline & $\vdash \Pi, P$ & $\operatorname{Pos}_{n}$
\end{tabular}

Let $\mathcal{D}\left(\pi_{i}\right)$ be the interpretation of $\pi_{i}$ in $\vdash \mathbf{\Pi}, \mathbf{P}_{\xi}, \mathbf{N}_{\sigma i}$, for $1 \leq i \leq n$. The strategy

$$
\mathcal{D}(\pi):=\left(\sigma, I_{n}\right)^{+} .\left\{\mathcal{D}_{1}, \ldots, \mathcal{D}_{n}\right\}[\xi / \sigma]
$$

is the interpretation of $\pi$ in $\vdash \mathbf{\Pi}, \mathbf{P}_{\xi}$.

Negative rule: $\pi$ ends with a negative rule $\operatorname{Neg}_{n}$, where $N=!_{N}\left(P_{1} \not 8 \ldots\right.$ \& $\left.P_{n}\right)$.

$$
\begin{gathered}
\vdots \pi_{0} \\
\frac{\vdash \Pi, P_{1}, \ldots, P_{n}}{\vdash \Pi, N} \mathrm{Neg}_{n}
\end{gathered}
$$

Let $\mathcal{D}\left(\pi_{0}\right)$ be the interpretation of $\pi_{0}$ in $\vdash \mathbf{\Pi}, \mathbf{P}_{\xi 1}, \ldots, \mathbf{P}_{\xi n}$. The strategy

$$
\mathcal{D}(\pi):=\left(\xi, I_{n}\right)^{-} \cdot \mathcal{D}\left(\pi_{0}\right)
$$

is the interpretation of $\pi$ in $\vdash \mathbf{\Pi}, \mathbf{N}_{\xi}$.

Cut: $\pi$ ends with a Cut rule:

$$
\begin{array}{cc}
\vdots \pi_{1} & \vdots \pi_{2} \\
\vdash \Xi, \Pi, P \quad \vdash \Delta, P^{\perp} \\
\vdash \Xi, \Pi, \Delta
\end{array}
$$

Let $\mathcal{D}\left(\pi_{1}\right)$ and $\mathcal{D}\left(\pi_{2}\right)$ be the interpretation of $\pi_{1}$ and $\pi_{2}$ in $\vdash \boldsymbol{\Xi}, \boldsymbol{\Pi}, \mathbf{P}_{\xi}$ and $\vdash \boldsymbol{\Delta}, \mathbf{P}_{\xi}^{\perp}$ respectively. The strategy

$$
\mathcal{D}(\pi):=\llbracket \mathcal{D}\left(\pi_{1}\right), \mathcal{D}\left(\pi_{2}\right) \rrbracket
$$

is the interpretation of $\pi$ in $\vdash \boldsymbol{\Xi}, \boldsymbol{\Pi}, \boldsymbol{\Delta}$. (Here we are assuming that the names in $\boldsymbol{\Pi}$ and $\Delta$ are disjoint.)

Example 11.15. Let us consider the case $n=0$ in the interpretation of the positive and negative rule respectively. Following the previous definition (with the same notation) we have:

- The strategy $(\xi, \emptyset)^{+}$is the interpretation in $\vdash \mathbf{\Pi}, ?_{P} \mathbf{1}_{\xi}$ of the derivation $\overline{\vdash \Pi, ?_{P} \mathbf{I}}$ Pos $_{0}$ (where the behaviour $?_{P} \mathbf{1}_{\xi}$ interprets the occurrence of the principal formula $?_{P} I$ ).

- The strategy $(\xi, \emptyset)^{-} \cdot \mathcal{D}(\rho)$ is the interpretation in $\vdash \Pi, !_{N} \perp_{\xi}$ of the derivation

$$
\begin{aligned}
& \vdots \rho \\
& \frac{\vdash \Pi}{\vdash \Pi, !_{N} \perp} \mathrm{Neg}_{0}
\end{aligned}
$$

11.4. Soundness. Having fixed the interpretations of formulas, sequents and derivations we are ready to show:

Theorem 11.16 (Soundness). Let $\pi$ be a derivation of a sequent $\vdash \Gamma$ in MELLS and $\mathcal{D}(\pi)$ be the interpretation of $\pi$ in a sequent of behaviours $\vdash \boldsymbol{\Gamma}$. We have that:

$$
\mathcal{D}(\pi) \text { is a winning strategy in } \vdash \boldsymbol{\Gamma} \text {. }
$$

Moreover, the interpretation is invariant under cut-elimination. 
The proof consists of several lemmas and it is given in Appendix B.

11.5. Full completeness. We can finally prove the following:

Theorem 11.17 (Full Completeness). Let $\vdash \Gamma$ be a sequent of behaviours which is interpretation of the sequent $\vdash \Gamma$ in MELLS. If $\mathcal{D}$ is a winning strategy in $\vdash \boldsymbol{\Gamma}$ then it is the interpretation of a cut-free derivation $\pi$ of the sequent $\vdash \Gamma$ in MELLS.

Proof. Since $\mathcal{D}$ is finite, we can reason by induction on the number $\nu$ of its actions. Let us examine the other implications of the winning conditions on $\mathcal{D}$. Since $\mathcal{D}$ is a daimon-free and deterministic strategy, all occurrences of actions in $\mathcal{D}$ are proper actions. Moreover, we have that $\mathcal{D}$ is non-empty, and has a single root (both properties always hold for positive strategies, and here also in case $\mathcal{D}$ is negative by Lemma 10.7 and 11.9, because $\mathcal{D}$ is material).

Like in the linear case, we will use - back and forth - the definition of sequent of behaviours and more precisely Proposition 10.6, Let $\vdash \boldsymbol{\Gamma}$ be the interpretation of the sequent $\vdash \Gamma$, and $\mathcal{D} \in \vdash \Gamma$ a winning strategy. Our purpose is to associate to $\mathcal{D}$ a derivation $\mathcal{D}^{\star}$ of $\vdash \Gamma$ in MELLS, by progressively decomposing $\mathcal{D}$, i.e., inductively writing "the last rule."

The formula on which the last rule is applied is indicated by the name of the root action. The argument is the same as in the linear case. For example, assume that the root of $\mathcal{D}$ is $(\xi, I)$; then if $\mathcal{D} \in \vdash \mathbf{F}_{\xi}, \mathbf{Q}_{\sigma}$, the behaviour which corresponds to the last rule is the one on $\xi$, i.e., $\mathbf{F}_{\xi}$.

In the sequel, we consider sequents of behaviours of the form $\vdash \mathbf{\Pi}, \mathbf{F}_{\xi}$, which are interpretations of a sequent $\vdash \Pi, F$ in MELLS. $\mathbf{F}$ is the interpretation of a formula $F$ and $\Pi$ is a sequence of $m$ behaviours $\mathbf{Q}_{\alpha_{1}}, \ldots, \mathbf{Q}_{\alpha_{m}}$ which respectively interpret formulas $Q_{1}, \ldots, Q_{m}$. Observe that $\boldsymbol{\Pi}$ always consists of positive behaviours only.

We use the following convention: we write $(\mathcal{E})$ and $(\mathcal{E}) \in \mathbf{C}$ for $\mathcal{E}_{1}, \ldots, \mathcal{E}_{m}$ and $\mathcal{E}_{1} \in$ $\mathbf{Q}_{\alpha_{1}}^{\perp}, \ldots, \mathcal{E}_{m} \in \mathbf{Q}_{\alpha_{m}}^{\perp}$ respectively.

We have two cases.

Positive case. Let $\mathcal{D}=\left(\xi, I_{n}\right)^{+} .\left\{\mathcal{D}_{1}, \ldots, \mathcal{D}_{n}\right\}(n \geq 0)$ be a positive winning strategy which belongs to $\vdash \boldsymbol{\Pi}, \mathbf{P}_{\xi}$, where $\mathbf{P}_{\xi}=\mathbf{F}^{+}\left(\mathbf{N}_{\xi 1}, \ldots, \mathbf{N}_{\xi k}\right)$ is the interpretation of the formula of MELLS $P=?_{P}\left(N_{1} \otimes \cdots \otimes N_{k}\right)$.

By Proposition 10.6 , for any $(\mathcal{E}) \in \mathbf{C}$, we have that $\mathcal{C}=\llbracket \mathcal{D},(\mathcal{E}) \rrbracket \in \mathbf{P}_{\xi}$. The root of $\llbracket \mathcal{D},(\mathcal{E}) \rrbracket$ is still $\left(\xi, I_{n}\right)^{+}$(by the definition of the abstract machine, since it is a visible action which is root of the main). Hence $k=n$.

We now use internal completeness.

(1) By internal completeness of positive connectives (Proposition 10.12), we have that the strategy $\underline{\sigma}(\llbracket \mathcal{D},(\mathcal{E}) \rrbracket)$ can be written as $\mathcal{C}_{1} \bullet \cdots \bullet \mathcal{C}_{n}$, where $\mathcal{C}_{i} \in \vdash \mathbf{P}_{\xi}, \mathbf{N}_{\sigma i}$.

(2) It is immediate that $\underline{\sigma}(\llbracket \mathcal{D},(\mathcal{E}) \rrbracket)=\llbracket \underline{\sigma}(\mathcal{D}),(\mathcal{E}) \rrbracket$. By Lemma 10.1, we can write $\underline{\sigma}(\mathcal{D})=$ $\left(\sigma, I_{n}\right)^{+} .\left\{\mathcal{D}_{1}^{\prime}, \ldots, \mathcal{D}_{n}^{\prime}\right\}$ as $\mathcal{D}_{1}^{\prime} \bullet \cdots \bullet \mathcal{D}_{n}^{\prime}$. By Lemma 10.2, we have that $\llbracket \mathcal{D}_{1}^{\prime} \bullet \cdots \bullet \mathcal{D}_{n}^{\prime},(\mathcal{E}) \rrbracket=$ $\llbracket \mathcal{D}_{1}^{\prime},(\mathcal{E}) \rrbracket \bullet \cdots \bullet \llbracket \mathcal{D}_{n}^{\prime},(\mathcal{E}) \rrbracket$.

From (1), we conclude that $\llbracket \mathcal{D}_{i}^{\prime},(\mathcal{E}) \rrbracket \in \vdash \mathbf{P}_{\xi}, \mathbf{N}_{\sigma i}$.

By applying Proposition 10.6 again, we have that $\mathcal{D}_{i}^{\prime} \in \vdash \mathbf{\Pi}, \mathbf{P}_{\xi}, \mathbf{N}_{\sigma i}$. 
If $\nu=1, \mathcal{D}$ consists of a single action $\left(\xi, I_{n}\right)^{+}$, and we must have $n=0$. Otherwise, we would have empty $\mathcal{D}_{i}^{\prime}$ 's, and we already observed that this cannot be the case. Hence, $\nu=1$ gives $\mathbf{P}_{\xi}=?_{P} \mathbf{1}$, and we have that $\mathcal{D}=(\xi, \emptyset)^{+}$is the interpretation of the rule $\operatorname{Pos}_{0}$.

If $\nu>1$, we note that the winning conditions are preserved for each $\mathcal{D}_{i}^{\prime}$ (by Lemma 11.18 and Lemma 11.19 below) and the number of actions decreases. Hence, the inductive hypothesis applies, and we can write the derivation:

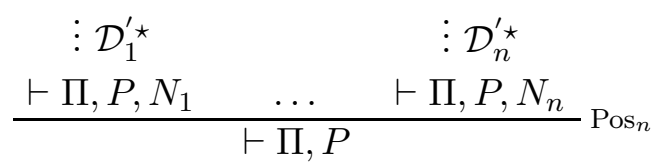

Negative case. Let us consider a negative winning strategy $\mathcal{D} \in \vdash \mathbf{\Pi}, \mathbf{N}_{\xi}$, where the behaviour $\mathbf{N}_{\xi}=\mathbf{F}^{-}\left(\mathbf{P}_{1}, \ldots, \mathbf{P}_{n}\right)$ is the interpretation of a formula $N=!_{N}\left(P_{1} \not 2 \ldots \not>P_{n}\right)$ of MELLS. Let $\mathcal{D}=x^{-} \cdot \mathcal{D}^{\prime}$, with $x=\left(\xi, I_{n}\right)$.

For any family $(\mathcal{E}) \in \mathbf{C}$, we have

(1) $\llbracket \mathcal{D},(\mathcal{E}) \rrbracket \in \mathbf{N}_{\xi}$, and the root is still $x^{-}$. This allows us to use internal completeness.

(2) By internal completeness of negative connectives (Proposition 10.8), we conclude that $\llbracket \mathcal{D},(\mathcal{E}) \rrbracket$ is of the form $x^{-} . \mathcal{D}^{\prime \prime}$ with $\mathcal{D}^{\prime \prime} \in \vdash \mathbf{P}_{\xi 1}, \ldots, \mathbf{P}_{\xi n}$.

(3) By the definition of normalization,

$$
\llbracket \mathcal{D},(\mathcal{E}) \rrbracket=\llbracket x^{-} \cdot \mathcal{D}^{\prime},(\mathcal{E}) \rrbracket=x^{-} \cdot \llbracket \mathcal{D}^{\prime},(\mathcal{E}) \rrbracket .
$$

From this, we have that $\mathcal{D}^{\prime \prime}=\llbracket \mathcal{D}^{\prime},(\mathcal{E}) \rrbracket$ and hence $\llbracket \mathcal{D}^{\prime},(\mathcal{E}) \rrbracket \in \vdash \mathbf{P}_{\xi 1}, \ldots, \mathbf{P}_{\xi n}$.

By applying Proposition [10.6] again, we have that $\mathcal{D}^{\prime} \in \vdash \boldsymbol{\Pi}, \mathbf{P}_{\xi 1}, \ldots, \mathbf{P}_{\xi n}$. The winning conditions are preserved for $\mathcal{D}^{\prime}$ (Lemma 11.20 below) and the number of actions decreases. Hence, the inductive hypothesis applies. Then, we can write the derivation:

$$
\begin{aligned}
& \vdots \mathcal{D}^{\prime \star} \\
& \frac{\vdash \Pi, P_{1}, \ldots, P_{n}}{\vdash \Pi, N} \mathrm{Neg}_{n}
\end{aligned}
$$

We still have to prove that the winning conditions are preserved by the deconstructions we have used in the last proof. The only condition which is not obvious is materiality.

To show that a strategy $\mathcal{D}$ is material in $\vdash \boldsymbol{\Gamma}$, we check that for each $s \in \mathcal{D}$ there is a certain family of counter-strategies $(\mathcal{E}) \in \mathbf{C}$ such that $s=\ulcorner p\urcorner$ and $p \in I(\mathcal{D},(\mathcal{E}))$. In other words, $s$ is used to produce the normal form $\llbracket \mathcal{D},(\mathcal{E}) \rrbracket$ (see Definitions 11.5 and 11.2).

Lemma 11.18. Let $\mathbf{P}_{\xi}=\mathbf{F}^{+}\left(\mathbf{N}_{\xi 1}, \ldots, \mathbf{N}_{\xi n}\right), \mathcal{D} \in \vdash \mathbf{\Pi}, \mathbf{P}_{\xi}$ and $\underline{\sigma}(\mathcal{D}) \in \vdash \boldsymbol{\Pi}, \mathbf{P}_{\xi}, \mathbf{P}_{\sigma}$. If $\mathcal{D}$ is material in $\vdash \mathbf{\Pi}, \mathbf{P}_{\xi}$ then $\underline{\sigma}(\mathcal{D})$ is material in $\vdash \mathbf{\Pi}, \mathbf{P}_{\xi}, \mathbf{P}_{\sigma}$.

Proof. W.l.o.g., we assume $\boldsymbol{\Pi}=\mathbf{Q}_{\alpha}$. If $\mathcal{D}: \alpha^{+}, \xi^{+}$is material, it means that for each $t \in \mathcal{D}$, we have $t=\ulcorner q\urcorner$, with $q \in I(\mathcal{D}, \mathcal{E}, \mathcal{A})$ for a certain $\mathcal{E} \in \mathbf{P}_{\xi}^{\perp}$ and a certain $\mathcal{A} \in \mathbf{Q}_{\alpha}^{\perp}$.

Let us consider $\mathcal{R}=\{\underline{\sigma}(\mathcal{D}), \mathcal{E}, \mathcal{E}[\sigma / \xi], \mathcal{A}\}$ and $\mathcal{R}^{\prime}=\{\mathcal{D}, \mathcal{E}, \mathcal{A}\}$. As discussed in the proof of Proposition 8.14, $p \in I(\mathcal{R})$ if and only if there is a "corresponding" $p^{\prime} \in I\left(\mathcal{R}^{\prime}\right)$, where $p^{\prime}$ is obtained from $p$ by the operation of renaming of pointing strings described in the proof of Proposition 8.14.

Now, let us consider a view $s \in \underline{\sigma}(\mathcal{D}): \alpha^{+}, \xi^{+}, \sigma^{+}$. We have that $s^{\prime} \in \mathcal{D}$ (where $s^{\prime}$ is obtained from $s$ by the same operation of renaming), and since $\mathcal{D}$ is material, $s^{\prime}=\ulcorner q\urcorner$ for 
$q \in I(\mathcal{D}, \mathcal{E}, \mathcal{A})$, where $\mathcal{E}$ and $\mathcal{A}$ are those provided by the materiality of $\mathcal{D}$. Let $p$ be the pointing string such that $q=p^{\prime}$. We conclude that $s=\ulcorner p\urcorner$, for $p \in I(\underline{\sigma}(\mathcal{D}), \mathcal{E}, \mathcal{E}[\sigma / \xi], \mathcal{A})$.

Lemma 11.19. Let $\mathbf{P}_{\sigma}=\mathbf{F}^{+}\left(\mathbf{N}_{\sigma 1}, \ldots, \mathbf{N}_{\sigma n}\right), \mathcal{D}=\mathcal{D}_{1} \bullet \ldots \bullet \mathcal{D}_{n} \in \vdash \mathbf{\Pi}, \mathbf{P}_{\sigma}$, and $\mathcal{D}_{i} \in \vdash$ $\boldsymbol{\Pi}, \mathbf{N}_{\sigma i}$. If $\mathcal{D}_{1} \bullet \cdots \bullet \mathcal{D}_{n}$ is material in $\vdash \mathbf{\Pi}, \mathbf{P}_{\sigma}$, then each $\mathcal{D}_{i}$ is material in $\vdash \mathbf{\Pi}, \mathbf{N}_{\sigma i}$.

Proof. For concreteness, we only discuss the case $\boldsymbol{\Pi}=\mathbf{Q}$, and $n=2$ (the general case being a simple generalization). Let $\mathcal{D}=x^{+} .\left\{\mathcal{D}_{1}, \mathcal{D}_{2}\right\}$ (we recall that $x$ occurs linearly in $\mathcal{D}$ ). For each $x^{-} . \mathcal{E} \in \mathbf{P}_{\sigma}^{\perp}$, we have by internal completeness (Proposition 10.8) that $\mathcal{E} \in \vdash \mathbf{N}_{\sigma 1}^{\perp}, \mathbf{N}_{\sigma 2}^{\perp}$; moreover for each $\mathcal{A} \in \mathbf{Q}^{\perp}, \llbracket \mathcal{D}_{i}, \mathcal{A} \rrbracket \in \mathbf{N}_{\sigma_{i}}$.

Let $s \in \mathcal{D}_{1}$. By construction, $x^{+} . s$ is a view in $\mathcal{D}$, and since $\mathcal{D}$ is material, $x^{+} . s$ belongs to $\mathcal{D}\left[x^{-} \cdot \mathcal{E}, \mathcal{A}\right]$, for a certain $x^{-} . \mathcal{E} \in \mathbf{P}_{\sigma}^{\perp}$ and a certain $\mathcal{A} \in \mathbf{Q}^{\perp}$ (we restrict our attention to single-rooted strategies in $\mathbf{P}_{\sigma}^{\perp}$, thanks to Lemma 10.10). It is then straightforward to check that if $x^{+} . s$ is used in the interaction which produces $\llbracket x^{+} .\left\{\mathcal{D}_{1}, \mathcal{D}_{2}\right\}, x^{-} \cdot \mathcal{E}, \mathcal{A} \rrbracket$, then $s$ is used in the interaction which produces $\llbracket \mathcal{D}_{1}, \mathcal{F}, \mathcal{A} \rrbracket$, where $\mathcal{F}:=\llbracket \mathcal{E}, \mathcal{D}_{2}, \mathcal{A} \rrbracket \in \mathbf{N}_{\sigma 1}^{\perp}$.

Lemma 11.20. Let $\mathbf{N}_{\xi}=\mathbf{F}^{-}\left(\mathbf{P}_{\xi 1}, \ldots, \mathbf{P}_{\xi n}\right)$. If $x^{-} . \mathcal{D} \in \vdash \mathbf{\Pi}, \mathbf{N}_{\xi}$ is material, then $\mathcal{D} \in \vdash$ $\Pi, \mathbf{P}_{\xi 1}, \ldots, \mathbf{P}_{\xi n}$ is material.

Proof. The argument is similar to the one for Lemma B.1(2), or rather it is its inverse. The only subtlety is that to ensure that the root of the counter-strategy we fix in $\mathbf{N}_{\xi}^{\perp}$ is linear, we need to go through some lengthy but straightforward steps of renaming (which we omit here) by repeated use of Proposition 8.14.

\section{Conclusion}

In this work, we started by recalling the standard notion of HO strategy and we have shown how ludics strategies can be expressed in term HO strategies by giving an universal arena. We have revised the main results of the higher-level part of ludics (namely, internal completeness) giving direct proofs of them using basic properties of the dynamics only. We have motivated and introduced the notion of non-uniform strategy and shown that we still have a suitable form of internal completeness when strategies are non-linear and non-uniform. From this, we finally have shown a full completeness result with respect to MELLS.

\section{Related and future work.}

Maurel's exponentials. Maurel [32] has built a sophisticated setting to recover a form of separation when having repetitions in ludics; however, the complexity of the setting prevented him from going further and studying interpretation and full completeness issues. In this paper, we ignore separation all together, and in fact we show that we don't need it in order to have interactive types and internal completeness. In future work, we hope it may be possible to refine our setting by using Maurel's techniques. In Maurel's setting, strategies have a quantitative information carried by probabilistic values (called coefficients). The values in the coefficients have a central role, and must satisfy a set of "quantitative conditions" inspired by measure theory. This is fundamentally different from our "indexed silent actions", as the specific natural number which is chosen as index for a silent action is irrelevant (in particular, all the indexes can be interchanged, and this does not affect orthogonality), and 
there are no condition attached. Our indexed silent actions have the same role as in [41]. However, in a way, we think that our approach could be seen as a simplification - or rather a kind of quotient - on Maurel's coefficients; on this grounds, we hope it may be possible to refine our silent actions by attaching probabilities to them, without losing our high-level results.

AJM style exponentials for ludics. A different solution that uses AJM style exponentials has been studied by the first of the two authors in [4]. Essentially, the strategies which inhabit a semantical type $!_{N} \mathbf{A}$ are those of the form $(\mathcal{D}, 1) \cup(\mathcal{E}, 2) \cup \ldots$ : an indexed (and disjoint) superimposition of strategies $\mathcal{D}, \mathcal{E}, \ldots$ of $\mathbf{A}$. However, the approach we use in this paper, which exploits similar ideas - namely the disjoint superimposition which is technically implemented by using silent actions here - is considerably simpler, as we do not need to consider further (rather complex) "uniformity" conditions to discriminate those strategies which are interpretations of syntactical derivations; here the "uniform strategies" are simply the deterministic strategies. We think that the approach to repetitions we implement in this paper is more suitable for more applicative uses of ludics [15, 38, 39].

Computational ludics. By using the approach we present in this paper, Basaldella and Terui [6] have recently extended Terui's computational ludics [39] in order to accommodate exponentials.

Their paper is aimed at analyzing the traditional logical duality between proofs and models from the point of view of ludics and they get an alternative proof of full completeness based on a direct construction of a counter-model. Very interestingly, that work also reveals an exciting property of the "interactive types." Unlike in standard HO game semantics, finiteness does not need to be requested as a condition for strategies to be winning; it is rather an outcome of the closure by orthogonality. In fact, Basaldella and Terui show that any material, deterministic strategy in a behaviour which is interpretation of logical formula is finite. We are confident that this result is also valid our setting. However, a careful verification of all the details is needed.

Non-deterministic innocent strategies. They have been introduced by Harmer in 24, with the purpose of modeling non-determinism (in PCF with erratic choice).

In this paper we introduce non-uniform strategies, which are realized by means of nondeterministic sums. However, the purpose of our non-deterministic sums is to implement non-uniformity via "formal sums" of strategies, in order to provide enough tests to make possible the interactive approach of ludics. The different purpose is reflected in the composition, which is simpler in our setting, where is in fact reduced to deterministic composition. Our strategies could be seen as a "concrete" implementation of Harmer's solution, in a simplified setting. Harmer overcomes the problems with composition moving from naive non-deterministic strategies $S: A \rightarrow B$ to an "indirect" definition of strategies of the kind $S: A \times \mathbb{N} \rightarrow B$. We have instead silent actions, which can be seen as $\tau$ actions carrying an index $i \in \mathbb{N}$. These actions have a two-fold role: they guard the sum (as in [41]), and provide an "index of copy" (as in AJM game semantics, but here the index is unfold only when needed), but do not go so far as to model non-determinism. In particular, we do not introduce any quotient on the strategies. 
Game semantics for linear logic. When we work with innocent strategies, in this paper we consider the variant of $\mathrm{HO}$ strategies introduced in [31. Having now enriched the setting of ludics with duplicative features, we want to make comparisons with other kinds of game semantics for linear logic. In particular, we are interested in finding connections between our treatment of duplication and the resource modalities of games semantics introduced by Melliès and Tabareau in [33].

Abstract machines. Curien and Herbelin in [11] have studied composition of strategies as sets of views. In particular they have developed the View-Abstract-Machine (VAM) (see also [8]) which is the device we use in this paper.

\section{ACKNOWLEDGEMENT}

We are in debt with Olivier Laurent for his sharp remarks and detailed suggestions which significantly contributed to improve an earlier version of our work. Many thanks to PierreLouis Curien, Kazushige Terui, Mauro Piccolo, for fruitful discussions and helpful suggestions. Finally, we gratefully acknowledge the anonymous referees whose in depth revision and many detailed comments have given an invaluable aid in improving the quality of this paper.

\section{REFERENCES}

[1] Abramsky, S.: Axioms for definability and full completeness. In: Proof, Language, and Interaction (Essay in honor of Robin Milner) The MIT Press (2000) 55-76.

[2] Abramsky, S., Jagadeesan, R., Malacaria, P.: Full Abstraction for PCF. Inf. Comput. 163(2) (2000) 409-470.

[3] Andreoli, J.-M.: Logic Programming with Focusing Proof in Linear Logic. J. Log. Comput. 2(3) (1992) 297-347.

[4] Basaldella, M.: On Exponentials in Ludics. PhD Thesis (2008) University of Siena.

[5] Basaldella, M., Faggian, C.: Ludics with Repetitions (Exponentials, Interactive Types and Completeness). In: LICS. (2009) 375-384.

[6] Basaldella, M., Terui, K.: On the meaning of logical completeness. Logical Methods in Computer Science 6(4:11) (2010) 1-35.

[7] Coquand, T.: A semantics of evidence for classical arithmetic. J. Symb. Log. 60(1) (1995) 325-337.

[8] Curien, P.-L.: Abstract Böhm trees. Math. Struct. in Comp. Sci. 8(6) (1998) 559-591.

[9] Curien, P.-L.: Introduction to Linear Logic and Ludics, part II. Advances of Mathematics (China) 35(1) (2006) 1-44.

[10] Curien, P.-L.: Notes on game semantics. Manuscript (2006).

[11] Curien, P.-L., Herbelin, H.: Abstract machines for dialogue games. Panoramas et Synthèses 27 (2009) 231-275.

[12] Faggian, C.: Travelling on designs. In: CSL. (2002) 427-441.

[13] Faggian, C., Fleury, M.-R., Quatrini, M.: An introduction to uniformity in Ludics. In: Linear logic in computer science, London Math. Soc. Lecture Note Ser., 316, Cambridge Univ. Press, Cambridge, (2004) 236-246.

[14] Faggian, C., Hyland, J.M.E.: Designs, disputes and strategies. In : CSL. (2002) 442-457.

[15] Faggian, C., Piccolo, M.: Ludics is a model for the finitary linear pi-calculus. In: TLCA. (2007) $148-162$.

[16] Faggian, C., Piccolo, M.: Partial Orders, Event Structures, and Linear Strategies. In: TLCA. (2009) 95-111.

[17] Girard, J.-Y.: Linear Logic. Theor. Comput. Sci. 50(1) (1987) 1-102.

[18] Girard, J.-Y.: Geometry of interaction I: Interpretation of System F. Logic Colloquium 88, In R. Ferro et al., (1989) 221-260. 
[19] Girard, J.-Y.: A New Constructive Logic: Classical Logic. Math. Struct. in Comp. Sci. 1(3) (1991) $255-296$.

[20] Girard, J.-Y.: On the meaning of logical rules I: syntax vs. semantics. Computational Logic (U. Berger and H. Schwichtenberg eds) Heidelberg Springer-Verlag (1999) 215 - 272.

[21] Girard, J.-Y.: On the meaning of logical rules II : multiplicatives and additives. Foundation of Secure Computation (2000) 183-212.

[22] Girard, J.-Y.: Locus solum: From the rules of logic to the logic of rules. Math. Struct. in Comp. Sci. 11(3) (2001) 301-506.

[23] Girard, J.-Y.: Le Point Aveugle, Cours de logique, Tome II: Vers l'imperfection. Visions des Sciences. Hermann (2007).

[24] Harmer, R. S.: Games and Full Abstraction for Nondeterministic Languages. PhD Thesis (1999) University of London.

[25] Harmer, R. S.: Innocent game semantics. Manuscript (2006).

[26] Hyland, J.M.E., Ong, C.H.L.: On full abstraction for PCF: I, II, and III. Inf. Comput. 163(2) (2000) $285-408$.

[27] Hyland, J.M.E., Schalk, A.: Glueing and orthogonality for models of linear logic. Theor. Comput. Sci. 294(1-2) (2003) 183-231.

[28] Krivine, J.-L.: Realizability in classical logic. Panoramas et Synthèses 27 (2009) 197-229.

[29] Laurent, O.: Étude de la polarization en logique. PhD thesis, Université Aix-Marseille II (2002).

[30] Laurent, O.: Polarized games. Ann. Pure Appl. Logic 130(1-3) (2004) 79-123.

[31] Laurent, O.: Syntax vs. semantics: A polarized approach. Theor. Comput. Sci. 343(1-2) (2005) 177-206.

[32] Maurel, F.: Un cadre quantitatif pour la Ludique. PhD Thesis (2004) Université Paris VII.

[33] Melliès, P.-A., Tabareau, N.: Resource modalities in game semantics. In: LICS. (2007) 389-398.

[34] Melliès, P.-A., Vouillon, J.: Recursive Polymorphic Types and Parametricity in an Operational Framework. In: LICS. (2005) 82-91.

[35] Nickau, H.: Hereditarily Sequential Functionals: A Game-Theoretic Approach to Sequentiality. PhD thesis, Universität GH Siegen (1996).

[36] Paolini, L.: Parametric $\lambda$-Theories. Theor. Comput. Sci. 398(1-3) (2008) 51-62.

[37] Pitts, A.M.: Parametric polymorphism and operational equivalence. Math. Struct. in Comp. Sci. 10(3) (2000) 321-359.

[38] Saurin, A.: Towards Ludics Programming: Interactive Proof Search. In: ICLP. (2008) 253-268.

[39] Terui, K.: Computational ludics. To appear in Theor. Comput. Sci. (2008).

[40] Yoshida, N., Berger, M., Honda, K.: Strong Normalisation in the pi-Calculus. In: LICS. (2001) 311-322.

[41] Varacca, D., Yoshida, N.: Typed Event Structures and the pi-Calculus: Extended Abstract. In: MFPS. (2006) 373-397.

\section{Appendix A. Admissibility of the Cut-rule and expressivity of MELLS}

In Section A.1 we prove that the cut-rule is admissible in MELLS.

We then discuss the expressivity of MELLS by relating it to more standard systems (in Sections A.2 and A.3). Since we are interested in clarifying the nature of the logical rules and sequents of MELLS, we omit the cut-rule in all system.

In the sequel we use extensively the following notions.

Definition A.1 (Depth, height). We define the depth of a formula $F$, noted by $d(F)$ as the length of the longest branch of $F$ in the obvious tree representation of $F$. More precisely, let $F$ be a formula in some language and $G_{1}, \ldots, G_{n}$ its immediate subformulas. We define $d(F)$ as the natural number inductively given by:

$$
d(F):=\sup \left\{d\left(G_{1}\right), \ldots, d\left(G_{n}\right)\right\}+1 .
$$

Let $\pi$ be a syntactical derivation of a sequent in some system. The height of $\pi$, noted by $h(\pi)$, is inductively given as follows: if $\pi$ ends with a rule whose premises are derived by 
$\pi_{1}, \ldots, \pi_{n}$, then

$$
h(\pi):=\sup \left\{h\left(\pi_{1}\right), \ldots, h\left(\pi_{n}\right)\right\}+1 .
$$

For instance, if $F$ (resp. $\pi$ ) is a formula (resp. derivation) in MELLS, we have that:

- $d(F)=1$ if and only if either $F=?_{P} \mid$ or $F=!_{N} \perp$;

- $h(\pi)=1$ if and only if $\pi$ is of the form $\frac{\vdash \Pi, ?_{P} I}{\text { Pos }_{0}}$ for some $\Pi$.

The following lemma is also useful in the sequel.

Proposition A.2 (Structural rules).

(1) Weakening: if $\vdash \Gamma$ is cut-free derivable then $\vdash \Gamma, Q$ is cut-free derivable.

(2) Contraction: if $\vdash \Gamma, Q, Q$ is cut-free derivable then $\vdash \Gamma, Q$ is cut-free derivable.

Proof.

(1) Let $\pi$ a be derivation of $\vdash \Gamma$. By induction on the height of $\pi$ we now construct a derivation $\pi^{\prime}$ of $\vdash \Gamma, Q$.

a. $\pi$ ends with a positive rule, where $P=?_{P}\left(N_{1} \otimes \cdots \otimes N_{n}\right)$ :

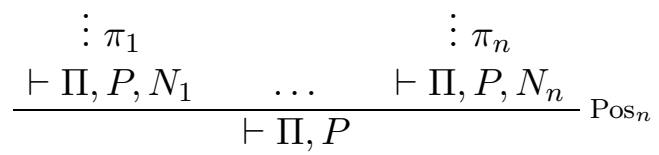

By inductive hypothesis, $\pi_{i}^{\prime}$ is a derivation of $\vdash \Pi, Q, P, N_{i}$ for any $1 \leq i \leq n$. We can then apply a rule $\operatorname{Pos}_{n}$ and obtain

$$
\begin{array}{ccc}
\vdots \pi_{1}^{\prime} & \vdots \pi_{n}^{\prime} \\
\vdash \Pi, Q, P, N_{1} & \ldots & \vdash \Pi, Q, P, N_{n} \\
\hline \multicolumn{3}{c}{\vdash \Pi, Q, P}
\end{array}
$$

b. $\pi$ ends with a negative rule, where $N=!_{N}\left(P_{1} \not 8 \ldots\right.$ \& $\left.P_{n}\right)$ :

$$
\begin{gathered}
\vdots \rho \\
\frac{\vdash \Pi, P_{1}, \ldots, P_{n}}{\vdash \Pi, N} \mathrm{Neg}_{n}
\end{gathered}
$$

By inductive hypothesis, $\rho^{\prime}$ is a derivation of $\vdash \Pi, Q, P_{1}, \ldots, P_{n}$. We can then apply a rule $\mathrm{Neg}_{n}$ and obtain

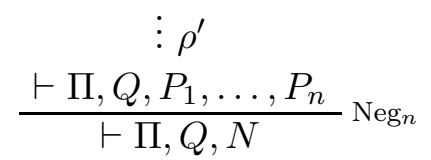

(2) Let $\pi$ a be derivation of $\vdash \Gamma, Q, Q$. By induction on the height of $\pi$ we now construct a derivation $\pi^{\prime}$ of $\vdash \Gamma, Q$.

a. $\pi$ ends with a positive rule, where the principal formula of $\operatorname{Pos}_{n}$ is (an occurrence of) some formula $P=?_{P}\left(N_{1} \underset{\vdots}{\otimes} \cdots \otimes N_{n}\right)$ in $\Pi, Q, Q: \quad \vdots \pi_{n}$

$$
\frac{\vdash \Pi, Q, Q, N_{1} \quad \ldots \quad \vdash \Pi, Q, Q, N_{n}}{\vdash \Pi, Q, Q} \operatorname{Pos}_{n}
$$

By inductive hypothesis, $\pi_{i}^{\prime}$ is a derivation of $\vdash \Pi, Q, N_{i}$ for any $1 \leq i \leq n$. Since $P$ also occurs in $\Pi, Q$, we can then apply $\operatorname{Pos}_{n}$ and obtain 


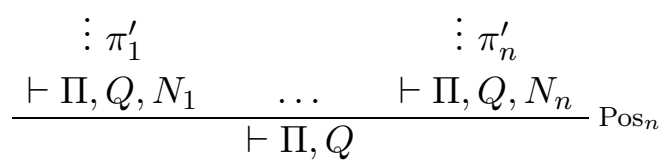

b. $\pi$ ends with a negative rule, where $N=!_{N}\left(P_{1} \not 8 \ldots 8 P_{n}\right)$ :

$$
\begin{gathered}
\vdots \rho \\
\frac{\vdash \Pi, Q, Q, P_{1}, \ldots, P_{n}}{\vdash \Pi, Q, Q, N} \mathrm{Neg}_{n}
\end{gathered}
$$

By inductive hypothesis, $\rho^{\prime}$ is a derivation of $\vdash \Pi, Q, P_{1}, \ldots, P_{n}$. We can then apply $\mathrm{Neg}_{n}$ rule and obtain

$$
\begin{gathered}
\vdots \rho^{\prime} \\
\frac{\vdash \Pi, Q, P_{1}, \ldots, P_{n}}{\vdash \Pi, Q, N} \mathrm{Neg}_{n}
\end{gathered}
$$

A.1. Admissibility of the Cut-rule. In this section we show that the cut-rule

$$
\frac{\vdash \Xi, \Pi, P \quad \vdash \Delta, P^{\perp}}{\vdash \Xi, \Pi, \Delta} \text { Cut }
$$

is admissible in MELLS (more precisely, the cut-rule is admissible in MELLS without Cut).

Theorem A.3 (Cut-elimination). Let $\pi$ and $\rho$ be cut-free derivations of $\vdash \Xi, \Pi, P$ and $\vdash \Delta, P^{\perp}$ respectively. The sequent $\vdash \Xi, \Pi, \Delta$ is derivable with a cut-free derivation $\theta$.

Proof. The proof by induction on the pair $(d(P), h(\pi)+h(\rho))$ - where $d(P)$ and $h(\pi)$ denote the depth of the formula $P$ and height of the derivation $\pi$ respectively (Definition A.1 ordered lexicographically: $(d(P), h(\pi)+h(\rho))<\left(d\left(P^{\prime}\right), h\left(\pi^{\prime}\right)+h\left(\rho^{\prime}\right)\right)$ if and only if either $d(P)<d\left(P^{\prime}\right)$ or $d(P)=d\left(P^{\prime}\right)$ and $h(\pi)+h(\rho)<h\left(\pi^{\prime}\right)+h\left(\rho^{\prime}\right)$.

Observe that we are assuming that $\pi$ and $\rho$ are cut-free derivations, this implies that the last rule of $\rho$ is always a negative rule having $P^{\perp}$ as principal formula.

We now give a procedure to obtain a cut-free derivation $\theta$ of $\vdash \Xi, \Pi, \Delta$. We have three distinct cases, depending on the last rule of $\pi$.

(a) The last rule of $\pi$ is a positive rule $\operatorname{Pos}_{n}$ and $P$ is principal in $\operatorname{Pos}_{n}$.

For readability, we only consider the cases $n=0$ and $n=2$. The general $n$-ary case straightforwardly follows.

If $n=0$, then $P=?_{P} \mathrm{I}, P^{\perp}=!_{N} \perp$ and $d(P)=1$. We have derivations:

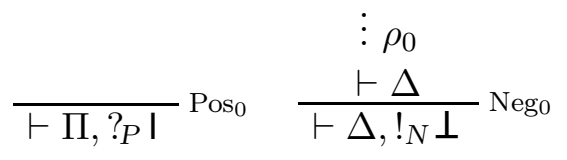

The cut-free derivation $\theta$ of $\vdash \Pi, \Delta$, is obtained from $\rho_{0}$ by weakening, in the sense of Proposition A.2 (1) (weakening on positive formulas).

If $n=2$, then $P=?_{P}\left(N_{1} \otimes N_{2}\right)$ and $P^{\perp}=!_{N}\left(N_{1}^{\perp} \not \gamma N_{2}^{\perp}\right)$. We have derivations: 


$$
\begin{aligned}
& \vdots \pi_{1} \quad \vdots \pi_{2} \quad \vdots \rho_{0} \\
& \frac{\vdash \Pi, P, N_{1} \quad \vdash \Pi, P, N_{2}}{\vdash \Pi, P} \operatorname{Pos}_{2} \quad \frac{\vdash \Delta, N_{1}^{\perp}, N_{2}^{\perp}}{\vdash \Delta, P^{\perp}} \mathrm{Neg}_{2}
\end{aligned}
$$

We now construct a cut-free derivation $\theta$ of $\vdash \Pi, \Delta$ as follows.

Consider for $i \in\{1,2\}$ the following derivations:

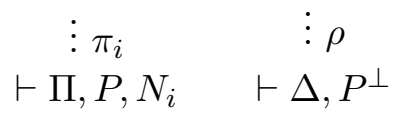

Since $h\left(\pi_{i}\right)<h(\pi)$, we have $\left(d(P), h\left(\pi_{i}\right)+h(\rho)\right)<(d(P), h(\pi)+h(\rho))$ and the induction hypothesis yields a cut-free derivation $\psi_{i}$ of $\vdash \Pi, \Delta, N_{i}$. Consider now

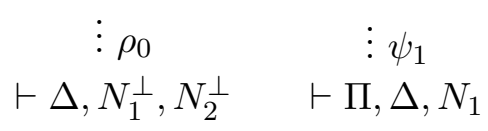

Since $N_{1}^{\perp}$ is an immediate subformula of $P$, we have $d\left(N_{1}^{\perp}\right)<d(P)$ and by induction we have a cut-free derivation $\theta_{1}$ of $\vdash \Pi, \Delta, \Delta, N_{2}^{\perp}$. Similarly, from $\theta_{1}$ and $\psi_{2}$ we get a cut-free derivation $\theta_{2}$ of $\vdash \Pi, \Pi, \Delta, \Delta, \Delta$. From $\theta_{2}$ we finally obtain a cut-free derivation $\theta$ of $\vdash \Pi, \Delta$ by repeatedly applying Proposition A.2 (2) (contraction on positive formulas).

(b) The last rule of $\pi$ is a positive rule $\operatorname{Pos}_{n}$ and $P$ is not principal in $\operatorname{Pos}_{n}$ :

$$
\begin{aligned}
& \vdots \pi_{1} \quad \vdots \pi_{n} \\
& \begin{array}{cc}
\vdash \Pi, P, Q, N_{1} \quad \ldots \quad \vdash \Pi, P, Q, N_{n} \\
\qquad \operatorname{Pos}_{n} \quad \vdash \Delta, P^{\perp}
\end{array}
\end{aligned}
$$

and the principal formula of $\operatorname{Pos}_{n}$ is the occurrence of formula $Q=?_{P}\left(N_{1} \otimes \cdots \otimes N_{n}\right)$. We now define a cut-free derivation $\theta$ of $\vdash \Pi, Q, \Delta$ as follows. For $1 \leq i \leq n$ consider pairs of derivations

$$
\begin{array}{cc}
\vdots \pi_{i} & \vdots \rho \\
\vdash \Pi, P, Q, N_{i} & \vdash \Delta, P^{\perp}
\end{array}
$$

Since $h\left(\pi_{i}\right)<h(\pi)$, we have $\left(d(P), h\left(\pi_{i}\right)+h(\rho)\right)<(d(P), h(\pi)+h(\rho))$ and by induction we get a cut-free derivation $\psi_{i}$ of $\vdash \Pi, Q, \Delta, N_{i}$. We now apply $\operatorname{Pos}_{n}$ to the sequents derived by $\psi_{1}, \ldots, \psi_{n}$, to obtain the cut-free derivation $\theta$ of $\vdash \Pi, Q, \Delta$ :

$$
\begin{array}{ccc}
\vdots \psi_{1} & \vdots \psi_{n} \\
\vdash \Pi, Q, \Delta, N_{1} & \ldots & \vdash \Pi, Q, \Delta, N_{n} \\
\hline \multicolumn{2}{c}{\vdash \Pi, Q, \Delta} & \operatorname{Pos}_{n}
\end{array}
$$

Notice that when $n=0$ and hence $Q=?_{P} \mid$ and $h(\pi)=1$, we have that 


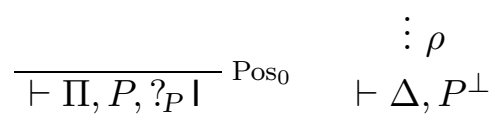

The procedure described above gives the cut-free derivation $\overline{\vdash \Pi, \Delta, ?_{P} I}{ }^{\text {Pos}_{0}}$.

(c) The last rule of $\pi$ is a negative rule $\mathrm{Neg}_{n}$, having $N=!_{N}\left(P_{1} \not \gamma \ldots \not 8 P_{n}\right)$ as principal formula.

$$
\begin{array}{cc}
\vdots \pi_{0} & \vdots \\
\frac{\vdash \Pi, P, P_{1}, \ldots, P_{n}}{\vdash \Pi, P, N} \mathrm{Neg}_{n} & \vdash \Delta, P^{\perp}
\end{array}
$$

To build a cut-free derivation of $\vdash N, \Pi, \Delta$, we first consider

$$
\begin{array}{cc}
\vdots \pi_{0} & \vdots \rho \\
\vdash \Pi, P, P_{1}, \ldots, P_{n} & \vdash \Delta, P^{\perp}
\end{array}
$$

Since $h\left(\pi_{0}\right)<h(\pi)$, we have $\left(d(P), h\left(\pi_{0}\right)+h(\rho)\right)<(d(P), h(\pi)+h(\rho))$ and by induction we get a cut-free derivation $\psi$ of $\vdash \Pi, P_{1}, \ldots, P_{n}, \Delta$. Applying $\mathrm{Neg}_{n}$, we finally get a cut-free derivation $\theta$ of $\vdash N, \Pi, \Delta$

$$
\begin{gathered}
\vdots \psi \\
\frac{\vdash \Pi, P_{1}, \ldots, P_{n}, \Delta}{\vdash N, \Pi, \Delta} \operatorname{Neg}_{n}
\end{gathered}
$$

By using the previous theorem, we have:

Corollary A.4. If $\vdash \Gamma$ is derivable in MELLS then $\vdash \Gamma$ is derivable in MELLS without Cut.

Proof. By induction on the height of a derivation $\pi$ of $\vdash \Gamma$. Suppose that $\pi$ ends with:

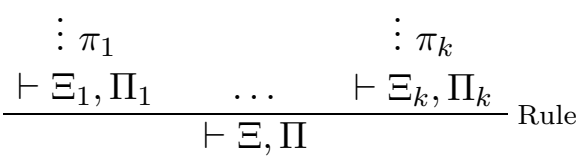

By inductive hypothesis, we have cut-free derivations $\psi_{i}$ of $\vdash \Xi_{i}, \Pi_{i}$, for any $1 \leq i \leq k$. If Rule is not Cut, we apply it to the sequents $\vdash \Xi_{i}, \Pi_{i}$ derived from $\psi_{i}$ and get a cut-free derivation of the conclusion. If Rule is Cut (and hence $k=2$ ), from the cut-free derivations $\psi_{1}$ and $\psi_{2}$ we get a cut-free derivation of the conclusion, by means of Theorem A.3.

A.2. MELLS related to the intuitionistic sequent calculus LJ. We show a correspondence between the calculus MELLS and a fragment of intuitionistic logic sequent calculus $\mathbf{L J}$, that we call $\mathbf{L} \mathbf{J}_{0}$, in which any formula is (hereditarily) a negation of a (possibly empty) conjunction of formulas. 
The main motivation for doing this is that in $\mathbf{L} \mathbf{J}_{0}$ we can employ the bilateral presentation of intuitionistic sequents - with at most one formula on the right side of the entailment symbol - to represent, in a more traditional way, the asymmetry between the polarized formulas of MELLS.

Formulas of $\mathbf{L} \mathbf{J}_{0}$ are given by the following grammar:

We write $\neg \top$ when $n=05$

$$
\mathrm{A}::=\neg\left(\mathrm{A}_{1} \wedge \cdots \wedge \mathrm{A}_{n}\right) \quad(n \geq 0) .
$$

Analogously to the standard calculus $\mathbf{L} \mathbf{J}$, a sequent of $\mathbf{L} \mathbf{J}_{0}$ is a pair of (possibly empty) multi-sets of formulas $\Pi$, 三, written $\Pi \vdash_{i n t} \equiv$, such that $\Xi$ contains at most one (occurrence of) formula.

The calculus $\mathbf{L} \mathbf{J}_{0}$ consists of two kinds of rules. They are given in Table 4 .

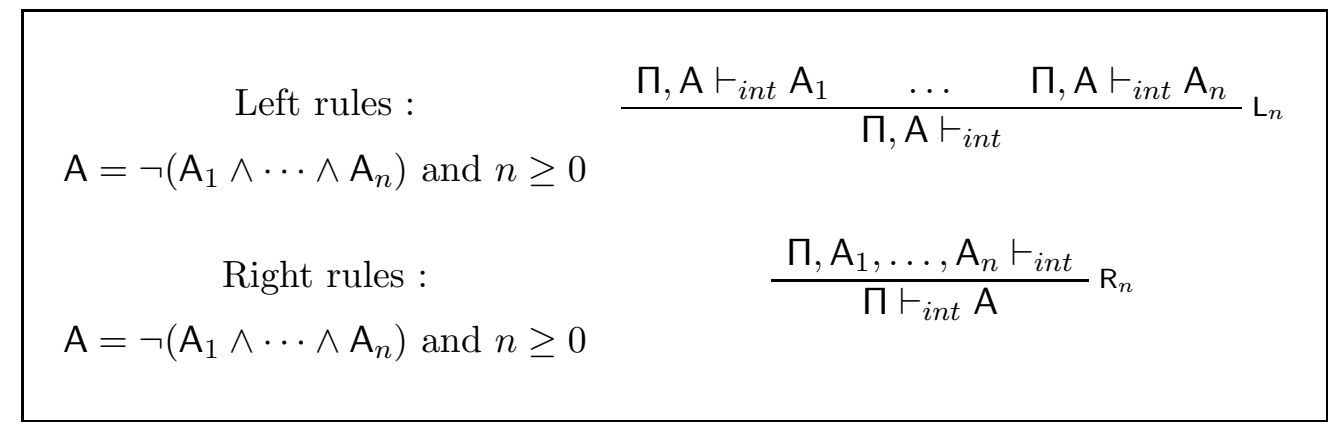

Table 4: $\mathbf{L J}_{0}$

In particular, when $n=0$ we have:

$$
\overline{\Pi, \neg \top \vdash_{i n t}} L_{0} \quad \frac{\Pi \vdash_{i n t}}{\Pi \vdash_{i n t} \neg \top} \mathrm{R}_{0}
$$

A.2.1. From MELLS to $\mathbf{L} \mathbf{J}_{0}$. The translation * of formulas of MELLS into formulas of $\mathbf{L} \mathbf{J}_{0}$ is given as follows.

$$
?_{P}\left(N_{1} \otimes \cdots \otimes N_{n}\right)^{*}:=\neg\left(N_{1}^{*} \wedge \cdots \wedge N_{n}^{*}\right) ; \quad !_{N}\left(P_{1} \not 8 \ldots>P_{n}\right)^{*}:=\neg\left(P_{1}^{*} \wedge \cdots \wedge P_{n}^{*}\right) .
$$

Given a multi-set $\Gamma=F_{1}, \ldots, F_{n}$ of formulas of of MELLS we denote by $\Gamma^{*}$ the multi-set $F_{1}^{*}, \ldots, F_{n}^{*}$ of formulas of $\mathbf{L} \mathbf{J}_{0}$.

Given a sequent $\vdash \Pi, \Xi$ of MELLS, where $\Pi$ is a multi-set of positive formulas and $\Xi$ is either empty of it consists of exactly of one (occurrence of) negative formula, we define $\vdash \Pi, \Xi^{*}:=\Pi^{*} \vdash_{i n t} \Xi^{*}$.

We have the following:

Proposition A.5. If $\vdash \Pi, \Xi$ is derivable in MELLS then $\vdash \Pi, \Xi^{*}$ is derivable in $\mathbf{L} \mathbf{J}_{0}$.

Proof. By induction on the height of the derivation $\pi$ of $\vdash \Pi, \Xi$ in MELLS.

- $\pi$ ends with a positive rule, where $P=?_{P}\left(N_{1} \otimes \cdots \otimes N_{n}\right)$.

\footnotetext{
${ }^{5}$ We chose the symbol $\top$ because $\top$ usually denotes the nullary version of $\wedge$. Consistently, we write $\neg \top$ for the nullary version of $\neg\left(A_{1} \wedge \cdots \wedge A_{n}\right)$.
} 


$$
\frac{\vdash \Pi, P, N_{1} \quad \ldots \quad \vdash \Pi, P, N_{n}}{\vdash \Pi, P} \operatorname{Pos}_{n}
$$

By inductive hypothesis, we have derivable sequents

$$
\vdash \Pi, P, N_{i}^{*}=\Pi^{*}, \neg\left(N_{1}^{*} \wedge \cdots \wedge N_{n}^{*}\right) \vdash_{i n t} N_{i}^{*},
$$

for any $1 \leq i \leq n$. We can then apply $\mathrm{L}_{n}$-rule and obtain

$$
\frac{\Pi^{*}, \neg\left(N_{1}^{*} \wedge \cdots \wedge N_{n}^{*}\right) \vdash_{i n t} N_{1}^{*} \quad \cdots \quad \Pi^{*}, \neg\left(N_{1}^{*} \wedge \cdots \wedge N_{n}^{*}\right) \vdash_{i n t} N_{n}^{*}}{\Pi^{*}, \neg\left(N_{1}^{*} \wedge \cdots \wedge N_{n}^{*}\right) \vdash_{i n t}}\left\llcorner_{n}\right.
$$

Since $\vdash \Pi, P^{*}=\Pi^{*}, \neg\left(N_{1}^{*} \wedge \cdots \wedge N_{n}^{*}\right) \vdash_{\text {int }}$, we are done.

- $\pi$ ends with a negative rule, where $N=!_{N}\left(P_{1}>8 \ldots 8 P_{n}\right)$.

$$
\frac{\vdash \Pi, P_{1}, \ldots, P_{n}}{\vdash \Pi, N} \mathrm{Neg}_{n}
$$

By inductive hypothesis, the sequent

$$
\vdash \Pi, P_{1}, \ldots, P_{n}^{*}=\Pi^{*}, P_{1}^{*}, \ldots, P_{n}^{*} \vdash_{i n t}
$$

is derivable. We can then apply $\mathrm{R}_{n}$-rule and obtain

$$
\frac{\Pi^{*}, P_{1}^{*}, \ldots, P_{n}^{*} \vdash_{i n t}}{\Pi^{*} \vdash_{i n t} \neg\left(P_{1}^{*} \wedge \cdots \wedge P_{n}^{*}\right)} \mathrm{R}_{n}
$$

Since $\vdash \Pi, N^{*}=\Pi^{*} \vdash_{i n t} \neg\left(P_{1}^{*} \wedge \cdots \wedge P_{n}^{*}\right)$, we are done.

A.2.2. From $\mathbf{L J}_{0}$ to MELLS. We also define an inverse translation $\diamond$ as follows.

We first define two translations, noted by ${ }^{\mathrm{p}}$ and ${ }^{\mathrm{n}}$, from formulas of $\mathbf{L} \mathbf{J}_{0}$ to positive and negative formulas of MELLS respectively:

$$
\neg\left(A_{1} \wedge \cdots \wedge A_{n}\right)^{p}:=?_{P}\left(A_{1}^{\mathrm{n}} \otimes \cdots \otimes A_{n}^{\mathrm{n}}\right) ; \quad \neg\left(\mathrm{A}_{1} \wedge \cdots \wedge \mathrm{A}_{n}\right)^{\mathrm{n}}:=!_{N}\left(\mathrm{~A}_{1}^{\mathrm{p}} \not 8 \cdots \not \mathrm{A}_{n}^{\mathrm{p}}\right) .
$$

In particular, $\neg \top \mathrm{P}=?_{P} \mathrm{I}$. Notice also that $A^{\mathrm{p}}=\left(\mathrm{A}^{\mathrm{n}}\right)^{\perp}$.

Given a multi-set $\Gamma=A_{1}, \ldots, A_{n}$ of formulas of $\mathbf{L} \mathbf{J}_{0}$ we write $\Gamma^{\mathrm{p}}\left(\operatorname{resp} . \Gamma^{\mathrm{n}}\right)$ for $A_{1}^{\mathrm{p}}, \ldots, A_{n}^{\mathrm{p}}$ (resp. $\left.A_{1}^{\mathrm{n}}, \ldots, \mathrm{A}_{n}^{\mathrm{n}}\right)$. Given a sequent $\Pi \vdash_{i n t} \equiv$ of $\mathbf{L} \mathbf{J}_{0}$ we define $\Pi \vdash_{i n t} \equiv \diamond:=\vdash \Pi^{\mathrm{p}}, \Xi^{\mathrm{n}}$.

Notice that $\Pi \vdash_{i n t} \equiv \diamond$ is always a sequent of MELLS, since it contains at most one (occurrence of) negative formula $\Xi^{\mathrm{n}}$.

We have:

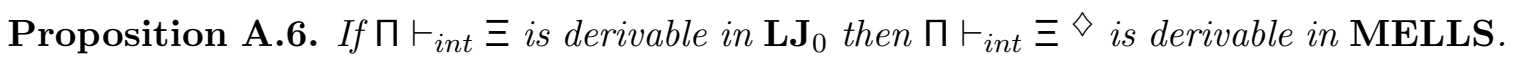

Proof. By induction on the height of a derivation $\pi$ of $\Pi \vdash_{i n t} \equiv$ in $\mathbf{L} \mathbf{J}_{0}$.

- $\pi$ ends with a left rule, where $A=\neg\left(A_{1} \wedge \cdots \wedge A_{n}\right)$.

$$
\frac{\Pi, \mathrm{A} \vdash_{i n t} \mathrm{~A}_{1} \quad \ldots \quad \Pi, \mathrm{A} \vdash_{i n t} \mathrm{~A}_{n}}{\Pi, \mathrm{A} \vdash_{i n t}}\left\llcorner_{n}\right.
$$

By inductive hypothesis, we have derivable sequents

$$
\Pi, \mathrm{A} \vdash_{i n t} \mathrm{~A}_{i} \diamond=\vdash \Pi^{\mathrm{p}}, ?_{P}\left(\mathrm{~A}_{1}^{\mathrm{n}} \otimes \cdots \otimes \mathrm{A}_{n}^{\mathrm{n}}\right), \mathrm{A}_{i}^{\mathrm{n}},
$$

for any $1 \leq i \leq n$. We can then apply $\operatorname{Pos}_{n}$-rule and obtain

$$
\frac{\vdash \Pi^{\mathrm{P}}, ?_{P}\left(\mathrm{~A}_{1}^{\mathrm{n}} \otimes \cdots \otimes \mathrm{A}_{n}^{\mathrm{n}}\right), \mathrm{A}_{1}^{\mathrm{n}} \quad \cdots \quad \vdash \Pi^{\mathrm{P}}, ?_{P}\left(\mathrm{~A}_{1}^{\mathrm{n}} \otimes \cdots \otimes \mathrm{A}_{n}^{\mathrm{n}}\right), \mathrm{A}_{n}^{\mathrm{n}}}{\vdash \Pi^{\mathrm{P}}, ?_{P}\left(\mathrm{~A}_{1}^{\mathrm{n}} \otimes \cdots \otimes \mathrm{A}_{n}^{\mathrm{n}}\right)} \operatorname{Pos}_{n}
$$

Since $\Pi, A \vdash_{i n t} \diamond=\vdash \Pi^{\mathrm{p}}, ?_{P}\left(\mathrm{~A}_{1}^{\mathrm{n}} \otimes \cdots \otimes \mathrm{A}_{n}^{\mathrm{n}}\right)$, we conclude the argument. 
- $\pi$ ends with a right rule, where $A=\neg\left(A_{1} \wedge \cdots \wedge A_{n}\right)$.

$$
\frac{\Pi, \mathrm{A}_{1}, \ldots, \mathrm{A}_{n} \vdash_{i n t}}{\Pi \vdash_{i n t} \mathrm{~A}} \mathrm{R}_{n}
$$

By inductive hypothesis, the sequent

$$
\Pi, \mathrm{A}_{1}, \ldots, \mathrm{A}_{n} \vdash_{\text {int }} \diamond=\vdash \Pi^{\mathrm{p}}, \mathrm{A}_{1}^{\mathrm{p}}, \ldots, \mathrm{A}_{n}^{\mathrm{p}}
$$

is derivable. Applying $\mathrm{Neg}_{n}$-rule we obtain

$$
\frac{\vdash \Pi^{\mathrm{p}}, \mathrm{A}_{1}^{\mathrm{p}}, \ldots, \mathrm{A}_{n}^{\mathrm{p}}}{\vdash \Pi^{\mathrm{P}}, !_{N}\left(\mathrm{~A}_{1}^{\mathrm{p}} \text { × } \ldots 2 \mathrm{~A}_{n}^{\mathrm{p}}\right)} \mathrm{Neg}_{n}
$$

Since $\Pi \vdash_{i n t} A \diamond=\vdash \Pi^{\mathrm{p}}, !_{N}\left(\mathrm{~A}_{1}^{\mathrm{p}} \not \supset \ldots \not \mathrm{A}_{n}^{\mathrm{p}}\right)$, we conclude the argument.

A.2.3. Composing ${ }^{*}$ and $\diamond$. We can finally show that the translations ${ }^{*}$ and $\diamond$ are the inverse of each other, in the sense we are now going to make precise.

We first show the following lemma.

\section{Lemma A.7.}

(1) For any positive formula $P$ of MELLS, we have $\left(P^{*}\right)^{\mathfrak{p}}=P$. Similarly, for any negative formula $N$, we have $\left(N^{*}\right)^{\mathrm{n}}=N$.

(2) For any formula $\mathrm{A}$ of $\mathbf{L} \mathbf{J}_{0}$, we have $\left(\mathrm{A}^{\mathrm{p}}\right)^{*}=\left(\mathrm{A}^{\mathrm{n}}\right)^{*}=\mathrm{A}$.

(3) For any sequent $\vdash \Gamma$ of MELLS, we have $\left(\vdash \Gamma^{*}\right)^{\diamond}=\vdash \Gamma$.

(4) For any sequent $\Pi \vdash_{\text {int }} \Xi$ of $\mathbf{L J}_{0}$, we have $\left(\Pi \vdash_{\text {int }} \Xi \diamond\right)^{*}=\Pi \vdash_{\text {int }} \Xi$.

Proof.

(1) By induction on the depth of $F$.

Let $P=?_{P}\left(N_{1} \otimes \cdots \otimes N_{n}\right)$. We have $\left(P^{*}\right)^{\mathrm{p}}=\neg\left(N_{1}^{*} \wedge \cdots \wedge N_{n}^{*}\right)^{\mathrm{p}}=?_{P}\left(\left(N_{1}^{*}\right)^{\mathrm{n}} \otimes\right.$ $\left.\cdots \otimes\left(N_{n}^{*}\right)^{\mathrm{n}}\right)$, and by inductive hypothesis we conclude the argument. Similarly, let $N=!_{N}\left(P_{1} \not 8 \ldots \not 8 P_{n}\right)$. We have $\left(N^{*}\right)^{\mathrm{n}}=\neg\left(P_{1}^{*} \wedge \cdots \wedge P_{n}^{*}\right)^{\mathrm{n}}=!_{N}\left(\left(P_{1}^{*}\right)^{\mathrm{p}} \not 8 \ldots \not 8\left(P_{n}^{*}\right)^{\mathrm{p}}\right)$, and again by inductive hypothesis we conclude the argument.

(2) By induction on the depth of $A=\neg\left(A_{1} \wedge \cdots \wedge A_{n}\right)$.

We have $\left(A^{\mathrm{p}}\right)^{*}=?_{P}\left(\mathrm{~A}_{1}^{\mathrm{n}} \otimes \cdots \otimes \mathrm{A}_{n}^{\mathrm{n}}\right)^{*}=\neg\left(\left(\mathrm{A}_{1}^{\mathrm{n}}\right)^{*} \wedge \cdots \wedge\left(\mathrm{A}_{n}^{\mathrm{n}}\right)^{*}\right)$, and by inductive hypothesis we conclude the argument. Similarly, $\left(A^{\mathrm{n}}\right)^{*}=!_{N}\left(\mathrm{~A}_{1}^{\mathrm{p}} \times 2 \ldots 8 \mathrm{~A}_{n}^{\mathrm{p}}\right)^{*}=\neg\left(\left(\mathrm{A}_{1}^{\mathrm{p}}\right)^{*} \wedge\right.$ $\left.\cdots \wedge\left(\mathrm{A}_{n}^{\mathrm{p}}\right)^{*}\right)$ and again by inductive hypothesis we conclude the argument.

(3) Let $\vdash \Gamma=\vdash P_{1}, \ldots, P_{n}, \Xi$. We have $\left(\vdash P_{1}, \ldots, P_{n}, \Xi^{*}\right)^{\diamond}=\left(P_{1}^{*}, \ldots, P_{n}^{*} \vdash_{\text {int }} \Xi^{*}\right)^{\diamond}=$ $\vdash\left(P_{1}^{*}\right)^{\mathrm{p}}, \ldots,\left(P_{n}^{*}\right)^{\mathrm{p}},\left(\Xi^{*}\right)^{\mathrm{n}}$, and by point $(1)$ above we conclude the argument.

(4) If $\Pi \vdash_{i n t} \Xi=\mathrm{A}_{1}, \ldots, \mathrm{A}_{n} \vdash_{\text {int }} \Xi$, we get $\left(\mathrm{A}_{1}, \ldots, \mathrm{A}_{n} \vdash_{i n t} \Xi \diamond\right)^{*}=\left(\vdash \mathrm{A}_{1}^{\mathrm{p}}, \ldots, \mathrm{A}_{n}^{\mathrm{p}}, \Xi^{\mathrm{n}}\right)^{*}=$ $\left(\mathrm{A}_{1}^{\mathrm{p}}\right)^{*}, \ldots,\left(\mathrm{A}_{n}^{\mathrm{p}}\right)^{*} \vdash_{\text {int }}\left(\Xi^{\mathrm{n}}\right)^{*}$, and by point $(2)$ above we conclude the argument.

Given a derivation $\pi$ of a sequent $\vdash \Pi, \Xi$ of MELLS we denote by $\pi^{*}$ the derivation of the sequent $\vdash \Pi, \Xi *$ of $\mathbf{L J}_{0}$ given by Proposition A.5. Similarly, given a derivation $\pi$ of a sequent $\Pi \vdash_{\text {int }} \equiv$ of $\mathbf{L} \mathbf{J}_{0}$ we denote by $\pi^{\diamond}$ the derivation of the sequent $\Pi \vdash_{\text {int }} \equiv \diamond$ of MELLS given by Proposition A.6.

We can finally show the following:

Theorem A.8.

(1) $\left(\pi^{*}\right)^{\diamond}=\pi$;

(2) $\left(\pi^{\diamond}\right)^{*}=\pi$. 
Proof.

(1) By induction on the height of the derivation $\pi$ of a sequent $\vdash \Pi, \Xi$ of MELLS.

Suppose that $\pi$ ends with

$$
\begin{array}{ccc}
\vdots \pi_{1} & & \vdots \pi_{n} \\
\vdash \Pi_{1}, \Xi_{1} & \ldots & \vdash \Pi_{n}, \Xi_{n} \\
\hline \multicolumn{3}{c}{\text { Rule }}
\end{array}
$$

By Proposition A.5, we get the derivation $\pi^{*}$ ending with

$$
\begin{aligned}
& \vdots \pi_{1}^{*} \quad \vdots \pi_{n}^{*} \\
& \frac{\Pi_{1}^{*} \vdash_{i n t} \Xi_{1}^{*} \quad \ldots \quad \Pi_{n}^{*} \vdash_{i n t} \Xi_{n}^{*}}{\Pi^{*} \vdash_{i n t} \Xi^{*}} \text { Rule }^{*}
\end{aligned}
$$

and by Proposition A.6 we finally get the derivation $\left(\pi^{*}\right)^{\diamond}$ ending with

$$
\begin{array}{cc}
\vdots\left(\pi_{1}^{*}\right)^{\diamond} & \vdots\left(\pi_{n}^{*}\right)^{\diamond} \\
\frac{\vdash\left(\Pi_{1}^{*}\right)^{\mathrm{p}},\left(\Xi_{1}^{*}\right)^{\mathrm{n}} \quad \ldots \quad \vdash\left(\Pi_{n}^{*}\right)^{\mathrm{p}},\left(\Xi_{n}^{*}\right)^{\mathrm{n}}}{\vdash\left(\Pi^{*}\right)^{\mathrm{p}},\left(\Xi^{*}\right)^{\mathrm{n}}}\left(\text { Rule }^{*}\right)^{\diamond}
\end{array}
$$

By Lemma A.7 (3), $\vdash\left(\Pi_{1}^{*}\right)^{\mathrm{p}},\left(\Xi_{1}^{*}\right)^{\mathrm{n}}=\vdash \Pi_{1}, \Xi_{1}, \ldots, \vdash\left(\Pi_{n}^{*}\right)^{\mathrm{p}},\left(\Xi_{n}^{*}\right)^{\mathrm{n}}=\vdash \Pi_{n}, \Xi_{n}$ and $\vdash\left(\Pi^{*}\right)^{\mathrm{p}},\left(\Xi^{*}\right)^{\mathrm{n}}=\vdash \Pi, \Xi$. It is immediate to verify that the principal and the auxiliary (occurrences of) formulas of Rule are exactly the same of (Rule*) $)^{\diamond}$, and hence the two expressions denote the same rule. By inductive hypothesis $\left(\pi_{1}^{*}\right)^{\diamond}=\pi_{1}, \ldots,\left(\pi_{n}^{*}\right)^{\diamond}=\pi_{n}$. We can finally conclude $\left(\pi^{*}\right)^{\diamond}=\pi$.

(2) By induction on the height of the derivation $\pi$ of a sequent $\Pi \vdash_{\text {int }} \equiv$ of $\mathbf{L} \mathbf{J}_{0}$.

Suppose that $\pi$ ends with

$$
\begin{aligned}
& \vdots \pi_{1} \quad \vdots \pi_{n} \\
& \frac{\Pi_{1} \vdash_{i n t} \Xi_{1} \quad \ldots \quad \Pi_{1} \vdash_{i n t} \Xi_{n}}{\Pi \vdash_{i n t} \equiv} \text { Rule }
\end{aligned}
$$

By Proposition A.6, we get the derivation $\pi^{\diamond}$ ending with

$$
\begin{array}{ccc}
\vdots \pi_{1}^{\diamond} & \vdots \pi_{n}^{\diamond} \\
\vdash \Pi_{1}^{\mathrm{p}}, \Xi_{1}^{\mathrm{n}} \quad \ldots \quad & \vdash \Pi_{n}^{\mathrm{p}}, \Xi_{n}^{\mathrm{n}} \\
\hline & \vdash \Pi^{\mathrm{p}}, \Xi^{\mathrm{n}} & \text { Rule }^{\diamond}
\end{array}
$$

and by Proposition A.5 we finally get the derivation $\left(\pi^{\diamond}\right)^{*}$ ending with

$$
\begin{aligned}
& \vdots\left(\pi_{1}^{\diamond}\right)^{*} \quad \vdots\left(\pi_{n}^{\diamond}\right)^{*} \\
& \frac{\left(\Pi_{1}^{\mathrm{p}}\right)^{*} \vdash_{i n t}\left(\Xi_{1}^{\mathrm{n}}\right)^{*} \quad \ldots \quad\left(\Pi_{n}^{\mathrm{p}}\right)^{*} \vdash_{i n t}\left(\Xi_{n}^{\mathrm{n}}\right)^{*}}{\left(\Pi^{\mathrm{p}}\right)^{*} \vdash_{i n t}\left(\Xi^{\mathrm{n}}\right)^{*}}\left(\mathrm{Rule}^{\diamond}\right)^{*}
\end{aligned}
$$

By Lemma A.7 (4), $\left(\Pi_{1}^{\mathrm{p}}\right)^{*} \vdash_{\text {int }}\left(\Xi_{1}^{\mathrm{n}}\right)^{*}=\Pi_{1} \vdash_{\text {int }} \Xi_{1}, \ldots,\left(\Pi_{n}^{\mathrm{p}}\right)^{*} \vdash_{i n t}\left(\Xi_{n}^{\mathrm{n}}\right)^{*}=\Pi_{n} \vdash_{\text {int }} \Xi_{n}$ and $\left(\Pi^{\mathfrak{p}}\right)^{*} \vdash_{i n t}\left(\Xi^{\mathrm{n}}\right)^{*}=\Pi \vdash_{i n t} \Xi$. As before, it is immediate to verify that the principal and the auxiliary (occurrences of) formulas of Rule are exactly the same of (Rule $)^{*}$, and hence they denote the same rule. By inductive hypothesis $\left(\pi_{1}^{\diamond}\right)^{*}=\pi_{1}, \ldots,\left(\pi_{n}^{\diamond}\right)^{*}=\pi_{n}$. We finally conclude $\left(\pi^{\diamond}\right)^{*}=\pi$. 
A.3. On the expressivity of MELLS. In this part, we discuss the relation between MELLS and some other (more standard) polarized variants of MELL (see also [6]).

A.3.1. $\mathbf{M E L L}_{\mathrm{pol}}$. We first recall the syntax of $\mathbf{M E L L}_{\mathrm{pol}}$ [29], the fragment of MELL given by the following data.

Formulas of $\mathbf{M E L L}_{\mathrm{pol}}$ are the polarized formulas given by the following grammar:

$$
\begin{aligned}
& \text { Positive formulas: } \mathrm{P}::=1|\mathrm{P} \otimes \mathrm{P}| \quad \text { !N } \\
& \text { Negative formulas: } \mathrm{N}::=\perp|\mathrm{N} \not 8 \mathrm{~N}| \text { ?P }
\end{aligned}
$$

Rules of MELL $_{\text {pol }}$ are the standard rules of MELL (Table 1) applied to sequents containing polarized formulas.

For our purposes, it is convenient to redefine the syntax of $\mathbf{M E L} \mathbf{L}_{\mathrm{pol}}$ by considering formulas in a certain canonical form, using the syntactical isomorphisms of linear logic

$$
\begin{array}{ll}
(A \otimes B) \otimes C \cong A \otimes(B \otimes C), & A \otimes 1 \cong A, \\
(A \ngtr B) \not \subset C \cong A>(B \ngtr C), & A \ngtr \perp \cong A .
\end{array}
$$

We redefine the formulas of $\mathbf{M E L L}_{\mathrm{pol}}$ as follows:

$$
\begin{array}{cccccc}
\text { Positive formulas: } & \mathrm{P}::=! \mathrm{M} & \mathrm{Q}::=\mathrm{P}_{1} \otimes \cdots \otimes \mathrm{P}_{n} \quad(n \geq 0) \\
\text { Negative formulas : } & \mathrm{N}::=? \mathrm{Q} & \mathrm{M}::=\mathrm{N}_{1} \not 2 \ldots \not \mathrm{N}_{n} \quad(n \geq 0)
\end{array}
$$

The constant 1 (resp. $\perp$ ) is given by $\mathrm{P}_{1} \otimes \cdots \otimes \mathrm{P}_{n}$ (resp. $\mathrm{N}_{1} \not 2 \ldots 8 \mathrm{~N}_{n}$ ) with $n=0$.

Notice that in this reformulation we now allow the unary tensor " $\otimes$ !M" and par "Х?Q." We also point out that $\otimes ! \mathrm{M}$ is different from $! \mathrm{M}$ as they have different outermost connectives. Similarly, 2 ?Q is different from ?Q. On the other hand, we do not have formulas like

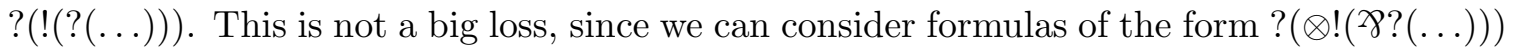
in place of them.

The rules are almost the same we gave for MELL. The only difference is that we here consider tensor and par rules of any arity $n \geq 0$. They are given in Table 5 .

$$
\begin{array}{ccc}
\text { Multiplicative rules } & \frac{\vdash \Gamma_{1}, \mathrm{P}_{1} \ldots}{\vdash \Gamma_{1}, \ldots, \Gamma_{n}, \mathrm{P}_{1} \otimes \cdots \otimes \mathrm{P}_{n}} \otimes_{n}, \mathrm{P}_{n} \\
& \frac{\vdash \Gamma, \mathrm{N}_{1}, \ldots, \mathrm{N}_{n}}{\vdash \Gamma, \mathrm{N}_{1} 8 \ldots \gamma \mathrm{N}_{n}} \ngtr_{n} \\
\text { Exponential and structural rules } & \frac{\vdash \mathrm{N}_{1}, \ldots, \mathrm{N}_{n}, \mathrm{M}}{\vdash \mathrm{N}_{1}, \ldots, \mathrm{N}_{n}, ! \mathrm{M}} ! \frac{\vdash \Gamma, \mathrm{Q}}{\vdash \Gamma, ? \mathrm{Q}} \\
& \frac{\vdash \Gamma}{\vdash \Gamma, \mathrm{N}} \mathrm{W} \frac{\vdash \Gamma, \mathrm{N}, \mathrm{N}}{\vdash \Gamma, \mathrm{N}} \mathrm{C}
\end{array}
$$

Table 5: MELL $_{\mathrm{pol}}$ 
The following lemmas are useful in the sequel.

Lemma A.9. If the sequent $\vdash \Gamma$ is derivable in $\mathbf{M E L L}_{\mathrm{pol}}$ then $\Gamma$ contains at most one occurrence of positive formulas.

Proof. By induction on the height of a derivation $\pi$ of $\vdash \Gamma$.

- Suppose that $\pi$ ends with a $\otimes_{n}$-rule. By inductive hypothesis, since $\mathrm{P}_{i}$ is positive, any $\Gamma_{i}$ consists of negative formulas only. Hence, in final sequent, $\mathrm{P}_{1} \otimes \cdots \otimes \mathrm{P}_{n}$ is the only occurrence of positive formula.

- Suppose that $\pi$ ends with a $\gamma_{n}$-rule. In this case, the number of occurrence of positive formulas in the premise is the same as in the conclusion.

- Suppose that $\pi$ ends with the !-rule. In this case, there is no occurrence of positive formula in the premise and exactly one in the conclusion.

- Suppose that $\pi$ ends with the ?-rule. In this case, there exactly one occurrence of positive formula in the premise and none in the conclusion.

- Suppose that $\pi$ ends with a structural rule $\mathrm{W}$ or $\mathrm{C}$. In this case the number of occurrence of positive formulas in the premise is the same as in the conclusion.

Lemma A.10. If $\vdash \Gamma, \mathrm{N}_{1} \not \supset \ldots \ngtr \mathrm{N}_{m}$ is derivable then $\vdash \Gamma, \mathrm{N}_{1}, \ldots, \mathrm{N}_{m}$ is derivable.

Proof. By induction on the height of the derivation $\pi$ of $\vdash \Gamma, \mathrm{N}_{1} \not \gamma \ldots \gamma \mathrm{N}_{m}$, we construct a derivation $\pi^{\prime}$ of $\vdash \Gamma, \mathrm{N}_{1}, \ldots, \mathrm{N}_{m}$ as follows.

- Suppose that $\pi$ ends with

$$
\begin{array}{cccc}
\vdots \pi_{1} & \vdots \pi_{i} & \vdots \\
\vdash \Gamma_{1}, \mathrm{P}_{1} \ldots & \vdash \Gamma_{i}, \mathrm{~N}_{1} \gamma \ldots \gamma \mathrm{N}_{m}, \mathrm{P}_{i} & \ldots & \vdash \Gamma_{n}, \mathrm{P}_{n} \\
\hline \multicolumn{2}{c}{\vdash \Gamma_{1}, \ldots, \Gamma_{i}, \mathrm{~N}_{1} \gamma \ldots \gamma \mathrm{N}_{m}, \ldots, \Gamma_{n}, \mathrm{P}_{1} \otimes \cdots \otimes \mathrm{P}_{n}}
\end{array} \otimes_{n}
$$

By inductive hypothesis, $\pi_{i}^{\prime}$ derives $\vdash \Gamma_{i}, \mathrm{~N}_{1}, \ldots, \mathrm{N}_{m}, \mathrm{P}_{i}$. We take

$$
\begin{aligned}
& \begin{array}{lll}
\vdots & \vdots & \vdots \\
1 & \vdots
\end{array} \pi_{n} \\
& \frac{\vdash \Gamma_{1}, \mathrm{P}_{1} \ldots \quad \vdash \Gamma_{i}, \mathrm{~N}_{1}, \ldots, \mathrm{N}_{m}, \mathrm{P}_{i} \quad \ldots \quad \vdash \Gamma_{n}, \mathrm{P}_{n}}{\vdash \Gamma_{1}, \ldots, \Gamma_{i}, \mathrm{~N}_{1}, \ldots, \mathrm{N}_{m}, \ldots, \Gamma_{n}, \mathrm{P}_{1} \otimes \cdots \otimes \mathrm{P}_{n}} \otimes_{n}
\end{aligned}
$$

- Suppose that $\pi$ ends with a $\gamma_{k}$-rule. We distinguish two subcases.

If $\mathrm{N}_{1} \not 8 \ldots 2 \mathrm{~N}_{m}$ is the principal formula in the last rule

$$
\begin{gathered}
\vdots \pi_{0} \\
\frac{\vdash \Gamma, \mathrm{N}_{1}, \ldots, \mathrm{N}_{m}}{\vdash \Gamma, \mathrm{N}_{1} \not 2 \ldots \mathrm{N}_{m}} \ngtr_{m}
\end{gathered}
$$

we take the derivation $\pi^{\prime}=\pi_{0}$.

Otherwise, $N_{1} 28 \ldots N_{m}$ is not the principal formula in the last rule

$$
\begin{aligned}
& \vdots \pi_{0} \\
& \frac{\vdash \Gamma, \mathrm{N}_{1} \not 8 \ldots \gamma \mathrm{N}_{m}, \mathrm{~N}_{1}^{\prime}, \ldots, \mathrm{N}_{n}^{\prime}}{\vdash \Gamma, \mathrm{N}_{1} \not 2 \ldots \not 8 \mathrm{~N}_{m}, \mathrm{~N}_{1}^{\prime} \not 2 \ldots 8 \mathrm{~N}_{n}^{\prime}} x_{n}
\end{aligned}
$$

By inductive hypothesis, $\pi_{0}^{\prime}$ derives $\vdash \Gamma, \mathrm{N}_{1}, \ldots, \mathrm{N}_{m}, \mathrm{~N}_{1}^{\prime}, \ldots, \mathrm{N}_{n}^{\prime}$. We take 


$$
\begin{gathered}
\vdots \pi_{0}^{\prime} \\
\frac{\vdash \Gamma, \mathrm{N}_{1}, \ldots, \mathrm{N}_{m}, \mathrm{~N}_{1}^{\prime}, \ldots, \mathrm{N}_{n}^{\prime}}{\vdash \Gamma, \mathrm{N}_{1}, \ldots, \mathrm{N}_{m}, \mathrm{~N}_{1}^{\prime} \not 2 \ldots \gamma \mathrm{N}_{n}^{\prime}} \ngtr_{n}
\end{gathered}
$$

- Suppose that $\pi$ ends with Rule $\in\{$ ?,W,C $\}$

$$
\begin{gathered}
\vdots \pi_{0} \\
\frac{\vdash \Gamma^{\prime}, \mathrm{N}_{1} \not \ldots \gamma \mathrm{N}_{m}}{\vdash \Gamma, \mathrm{N}_{1} \gamma \ldots \gamma \mathrm{N}_{m}} \text { Rule }
\end{gathered}
$$

By inductive hypothesis, $\pi_{0}^{\prime}$ derives $\vdash \Gamma^{\prime}, N_{1}, \ldots, N_{m}$. We take

$$
\begin{gathered}
\vdots \pi_{0}^{\prime} \\
\frac{\vdash \Gamma^{\prime}, \mathrm{N}_{1}, \ldots, \mathrm{N}_{m}}{\vdash \Gamma, \mathrm{N}_{1}, \ldots, \mathrm{N}_{m}} \text { Rule }
\end{gathered}
$$

We finally observe that there are no other cases: due to the presence of $N_{1} \not 8 \ldots \gamma 8 N_{m}$ in the final sequent, $\pi$ cannot end with the !-rule.

A.3.2. $\mathbf{M E L L}_{\mathrm{pol}}^{*}$. The next step is to consider the following subsystem of $\mathbf{M E L L} \mathbf{L}_{\mathrm{pol}}$ that we call $\mathbf{M E L L}_{\mathrm{pol}}^{*}$.

Formulas of $\mathbf{M E L L}_{\mathrm{pol}}^{*}$ are the same of $\mathbf{M E L L}_{\mathrm{pol}}$ but sequents are now multi-set of formulas of the form $\vdash \Delta=\vdash \mathrm{N}_{1}, \ldots, \mathrm{N}_{k-1}, \mathrm{~F}_{k}$, for some $k \geq 0$. In other words, a sequent of $\mathbf{M E L L}_{\text {pol }}^{*}$ contains at most one occurrence of formulas which is not a ?-formula. In some cases we also denote a sequent of $\mathbf{M E L L}_{\text {pol }}^{*}$ by $\vdash$ ? $\Gamma, \mathrm{F}$.

The rules of $\mathbf{M E L L} \mathbf{L}_{\mathrm{pol}}^{*}$ are the same of $\mathbf{M E L L} \mathbf{p o l}_{\mathrm{pol}}$, with the obvious modifications due to the constraint on sequents. They are given in Table 6 .

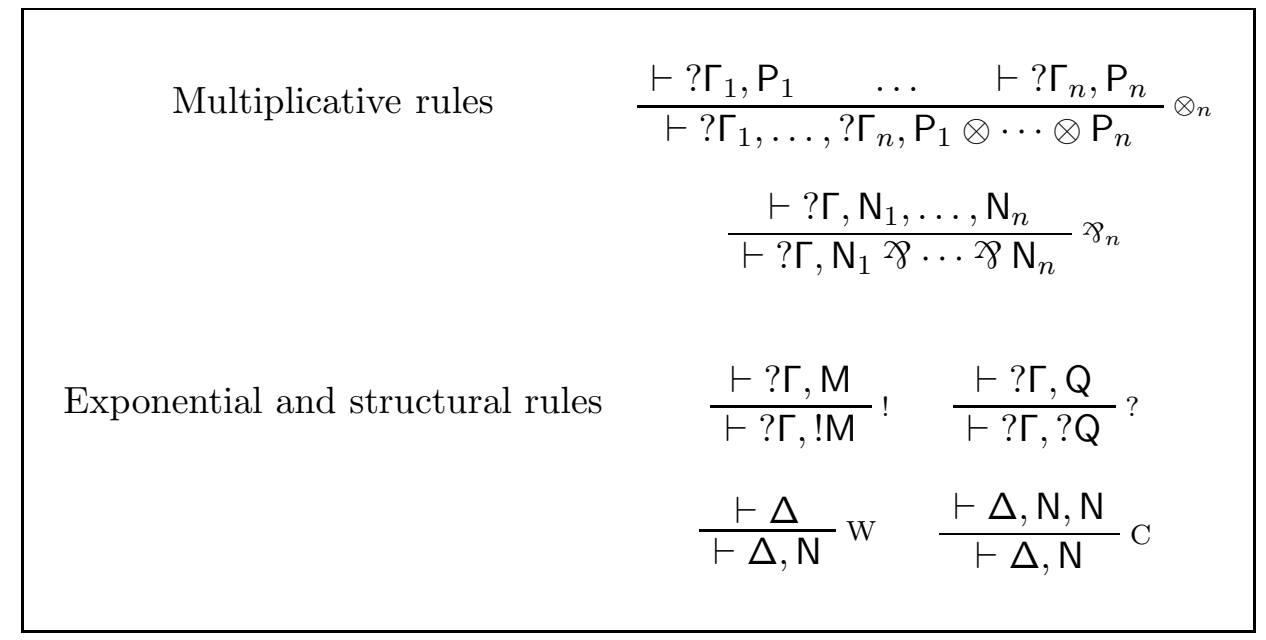

Table 6: $\mathbf{M E L L} \mathbf{L o l}_{\mathrm{pol}}$

The main consequence of restriction on the shape of sequents is that a sequent of the form 


$$
\vdash ? \Gamma, ? \mathrm{Q}, \mathrm{N}_{1} \gamma \ngtr \ldots \gamma \mathrm{N}_{n}
$$

cannot be inferred using the ?-rule on $Q$, as the resulting premise

$$
\vdash ? \Gamma, \mathrm{Q}, \mathrm{N}_{1}>2 \ldots>\mathrm{N}_{n}
$$

which is a sequent in $\mathbf{M E L L} \mathbf{p o l}_{\text {pol }}$, would not be a sequent of $\mathbf{M E L L}_{\mathrm{pol}}^{*}$.

Clearly, if $\vdash \Delta$ is derivable in $\mathbf{M E L L}_{\text {pol }}^{*}$, then $\vdash \Delta$ is derivable in $\mathbf{M E L L}_{\text {pol }}$. But we also have the converse.

Proposition A.11. If $\vdash \Delta$ is derivable in $\mathbf{M E L L}_{\mathrm{pol}}$ then $\vdash \Delta$ is derivable in $\mathbf{M E L L} \mathbf{p o l}_{\mathrm{pol}}^{*}$.

By induction on the height of a derivation $\pi$ of $\vdash \Delta$ in $\mathbf{M E L L}_{\text {pol }}$ we construct a derivation $\pi^{*}$ of $\vdash \Delta$ in $\mathbf{M E L L}_{\text {pol }}^{*}$. There are several cases to analyze.

Structural rules: $\pi$ ends with a structural rule Rule $\in\{\mathrm{W}, \mathrm{C}\}$ :

$$
\begin{aligned}
& \vdots \pi_{0} \\
& \frac{\vdash \Delta^{\prime}}{\vdash \Delta} \text { Rule }
\end{aligned}
$$

Since structural rules only affect ?-formulas, it is clear that $\vdash \Delta^{\prime}$ is a sequent of $\mathbf{M E L L} \mathbf{L}_{\text {pol }}^{*}$. The inductive hypothesis yields a derivation $\pi_{0}^{*}$ of the premise. We can then apply Rule and conclude.

Positive case: Suppose that $\vdash \Delta$ contains an (occurrence of) positive formula $F$. We have the following subcases.

- $\mathrm{F}=\mathrm{P}_{1} \otimes \cdots \otimes \mathrm{P}_{n}$ and $\pi$ ends with a $\otimes_{n}$-rule:

$$
\begin{aligned}
& \vdots \pi_{1} \quad \vdots \pi_{n} \\
& \frac{\vdash ? \Gamma_{1}, \mathrm{P}_{1} \quad \ldots \quad \vdash ? \Gamma_{n}, \mathrm{P}_{n}}{\vdash ? \Gamma_{1}, \ldots, ? \Gamma_{n}, \mathrm{P}_{1} \otimes \cdots \otimes \mathrm{P}_{n}} \otimes_{n}
\end{aligned}
$$

Since the premises are sequent of $\mathbf{M E L L}_{\mathrm{pol}}^{*}$, the inductive hypothesis yields the derivations $\pi_{1}^{*}, \ldots, \pi_{n}^{*}$ of the premises in $\mathbf{M E L L}_{\mathrm{pol}}^{*}$. We can then apply $\otimes_{n}$ and conclude.

- $\mathrm{F}=! \mathrm{M}$ and $\pi$ ends with the !-rule:

$$
\begin{gathered}
\vdots \pi_{0} \\
\vdash ? \Gamma, \mathrm{M} \\
\hline \vdash ? \Gamma, ! \mathrm{M} !
\end{gathered}
$$

Since $\vdash$ ?, $\mathrm{M}$ is a sequent of $\mathbf{M E L L} \mathbf{L}_{\text {pol }}^{*}$, the inductive hypothesis yields a derivation $\pi_{0}^{*}$ of the premise. We can then apply! and conclude.

There are no other cases, since the only possibility left out would be an inference of the form

$$
\frac{\vdash ? \Gamma^{\prime}, \mathrm{Q}, \mathrm{F}}{\vdash ? \Gamma^{\prime}, ? \mathrm{Q}, \mathrm{F}} ?
$$

but by Lemma A.9, $\vdash ? \Gamma^{\prime}, \mathrm{Q}, \mathrm{F}$ is not derivable in $\mathbf{M E L L} \mathbf{L}_{\mathrm{pol}}$, as it contains two occurrences of positive formula.

Negative case: Suppose that $\vdash \Delta$ contains no positive formula, so that $\vdash \Delta=\vdash$ ?, $\mathrm{F}$, where $\mathrm{F}$ is an (occurrence of) negative formula. We have the following subcases. 
- $\mathrm{F}=\mathrm{N}_{1} \not \gamma \ldots \gamma \mathrm{N}_{n}$. In such a case $\pi$ does not necessarily end with a $\gamma_{n}$-rule. For instance, the last rules of $\pi$ could be

$$
\frac{\vdash ? \Gamma^{\prime}, \mathrm{Q}, \mathrm{N}_{1}>8 \ldots 8 \mathrm{~N}_{n}}{\vdash ? \Gamma^{\prime}, ? \mathrm{Q}, \mathrm{N}_{1}>8 \ldots 8 \mathrm{~N}_{n}} x_{n}
$$

and $\vdash ? \Gamma^{\prime}, \mathrm{Q}, \mathrm{N}_{1} \not 8 \ldots 8 \mathrm{~N}_{n}$ is not a sequent of $\mathbf{M E L L} \mathbf{p o l}_{\mathrm{po}}^{*}$, as we have already discussed. We then proceed as follows.

Observe that the formula $\mathrm{N}_{1} \not 8 \ldots \gamma \mathrm{N}_{n}$ cannot be affected by means of structural rules, and the contexts of the $\otimes_{n}$-rules are splitting. Hence, there is a unique branch in $\pi$ where at some stage the formula $N_{1} \not \gamma \ldots \not 8 N_{n}$ is decomposed by means of a $\not{ }_{n}$-rule:

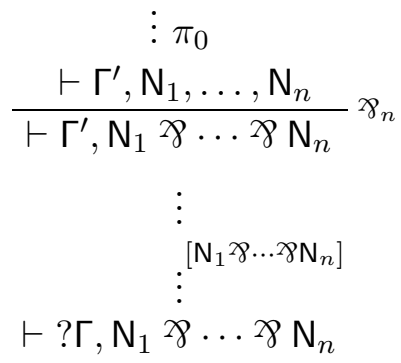

We also observe that $\vdash \Gamma^{\prime}, N_{1}, \ldots, N_{n}$ might not be a sequent of $\mathbf{M E L L}_{\text {pol }}^{*}$ and that there is no application of the !-rule in the branch.

Let $\rho$ be the proof-tree obtained from $\pi$ by replacing the previous branch with

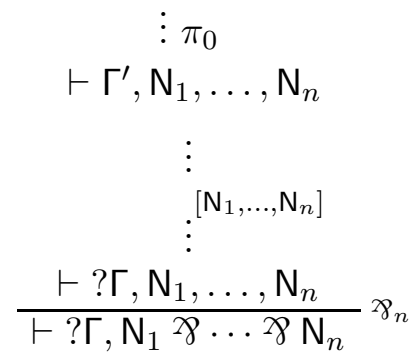

By Lemma A.10, $\rho$ is a correct derivation of $\vdash ? \Gamma, N_{1} \not \gamma \ldots \gamma \mathbf{N}_{n}$ in $\mathbf{M E L L} \mathbf{L}_{\text {pol }}$ ending with a $\gamma_{n}$-rule. Moreover, the height of $\rho$ is the same of $\pi$ because the new branch has the same height of the previous one. We can then apply the inductive hypothesis to the derivation, say $\rho_{0}$, of the premise $\vdash$ ? $, \mathrm{N}_{1}, \ldots, \mathrm{N}_{n}$ of the last inference rule of $\rho$. We obtain a derivation $\rho_{0}^{*}$ of $\vdash ? \Gamma, \mathrm{N}_{1}, \ldots, \mathrm{N}_{n}$ in $\mathbf{M E L L}_{\mathrm{pol}}^{*}$. To conclude, we apply $\gamma_{n}$. - $\mathrm{F}=$ ?Q and $\pi$ ends with the ?-rule:

$$
\begin{gathered}
\vdots \pi_{0} \\
\vdash ? \Gamma, \mathrm{Q} \\
\hline \vdash ? \Gamma, ? \mathrm{Q}
\end{gathered}
$$

Since $\vdash ? \Gamma, \mathrm{Q}$ is a sequent of $\mathbf{M E L L} \mathbf{L}_{\mathrm{po}}^{*}$, the inductive hypothesis yields a derivation $\pi_{0}^{*}$. We can then apply? and conclude the argument 
It is now possible to give a correspondence between MELLS and $\mathbf{M E L L}_{\text {pol }}^{*}$. We do this in the next section.

A.3.3. Correspondence with $\mathbf{M E L L} \mathbf{L}_{\mathrm{pol}}^{*}$. We first observe that any exponential formula (that is, a ?-formula or a !-formula) of $\mathbf{M E L L} \mathbf{p o l}_{\text {pol }}^{*}$ can be generated by the following grammar:

$$
\begin{array}{cc}
\text { Positive exponential formulas : } & \mathrm{P}::=!\left(\mathrm{N}_{1} \ngtr \ldots \times \mathrm{N}_{n}\right) \quad(n \geq 0) \\
\text { Negative exponential formulas : } & \mathrm{N}::=?\left(\mathrm{P}_{1} \otimes \cdots \otimes \mathrm{P}_{n}\right) \quad(n \geq 0)
\end{array}
$$

We can then define a translation ${ }^{\circ}$ from formulas of MELLS to exponential formulas of MELL $\mathbf{p o l}_{\text {pol }}^{*}$ recursively as follows:

$$
\begin{aligned}
& P^{\circ}=?_{P}\left(N_{1} \otimes \cdots \otimes N_{n}\right)^{\circ}:=?\left(N_{1}^{\circ} \otimes \cdots \otimes N_{n}^{\circ}\right) ; \\
& N^{\circ}=!_{N}\left(P_{1} \not 2 \cdots \ngtr P_{n}\right)^{\circ}:=!\left(P_{1}^{\circ} \not 2 \cdots \varnothing P_{n}^{\circ}\right) .
\end{aligned}
$$

Notice that the translation ${ }^{\circ}$ inverts the polarity. Given a multi-set of formulas $\Gamma=$ $F_{1}, \ldots, F_{n}$ of MELLS we write $\Gamma^{\circ}$ for the multi-set $F_{1}^{\circ}, \ldots, F_{n}^{\circ}$ of formulas of $\mathbf{M E L L}_{\text {pol }}^{*}$. Notice that, as a consequence of the restriction on the polarities for sequents of MELLS, $\vdash \Gamma^{\circ}$ is always a sequent of $\mathbf{M E L L}_{\mathrm{pol}}^{*}$, as it contains at most one occurrence of !-formula (all the remaining ones are ?-formulas).

Proposition A.12. If $\vdash \Gamma$ is derivable in MELLS then $\vdash \Gamma^{\circ}$ is derivable in $\mathbf{M E L L}_{\mathrm{pol}}^{*}$.

Proof. By induction on the height of a derivation $\pi$ of $\vdash \Gamma$ in MELLS.

- $\pi$ is $\overline{\vdash \Pi, ?_{P} I}$ Poso $_{0}$. We set:

$\frac{{\frac{\overline{\vdash 1}^{1}}{\vdash ? 1}}^{\text {؛ weakenings }}}{\frac{\vdash \Pi^{\circ}, ? 1}{}}$

- $\pi$ ends with a positive rule on $P=?_{P}\left(N_{1} \otimes \cdots \otimes N_{n}\right)$ (with $\left.n \geq 1\right)$ :

$$
\frac{\vdash \Pi, P, N_{1} \quad \ldots \quad \vdash \Pi, P, N_{n}}{\vdash \Pi, P} \operatorname{Pos}_{n}
$$

By inductive hypothesis, $\vdash \Pi^{\circ}, P^{\circ}, N_{i}^{\circ}$ is derivable for any $1 \leq i \leq n$. We set:

$$
\frac{\frac{\vdash \Pi^{\circ}, P^{\circ}, N_{1}^{\circ} \quad \ldots \quad \vdash \Pi^{\circ}, P^{\circ}, N_{n}^{\circ}}{\vdash \Pi^{\circ}, \ldots, \Pi^{\circ}, P^{\circ}, \ldots, P^{\circ}, N_{1}^{\circ} \otimes \cdots \otimes N_{n}^{\circ}} \otimes_{n}}{\frac{\vdash \Pi^{\circ}, \ldots, \Pi^{\circ}, P^{\circ}, \ldots, P^{\circ}, ?\left(N_{1}^{\circ} \otimes \cdots \otimes N_{n}^{\circ}\right)}{\vdots \text { contractions } \vdots}} ?
$$

- $\pi$ ends with a negative rule on $N=!_{N}\left(P_{1} \not 8 \ldots \not 8 P_{n}\right)$ (with $\left.n \geq 0\right)$ :

$$
\frac{\vdash \Pi, P_{1}, \ldots, P_{n}}{\vdash \Pi, !_{N}\left(P_{1} \text { × } \ldots \text { × } P_{n}\right)} \mathrm{Neg}_{n}
$$

By inductive hypothesis, $\vdash \Pi^{\circ}, P_{1}^{\circ}, \ldots, P_{n}^{\circ}$ is derivable and we set: 


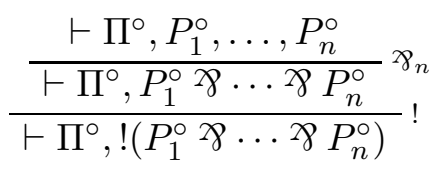

To show the converse, we define a translation ${ }^{-}$from formulas of $\mathbf{M E L L} \mathbf{L}_{\text {pol }}^{*}$ to formulas of MELLS recursively as follows:

$$
\begin{array}{ll}
\mathrm{P}^{\bullet}=\mathrm{M}^{\bullet}:=\mathrm{M}^{\bullet} ; & \mathrm{Q}^{\bullet}=\mathrm{P}_{1} \otimes \cdots \otimes \mathrm{P}_{n} \bullet:=?_{P}\left(\mathrm{P}_{1}^{\bullet} \otimes \cdots \otimes \mathrm{P}_{n}^{\bullet}\right) ; \\
\mathrm{N}^{\bullet}=? \mathrm{Q}^{\bullet}:=\mathrm{Q}^{\bullet} ; & \mathrm{M}^{\bullet}=\mathrm{N}_{1} \not 2 \cdots \not \mathrm{N}_{n}^{\bullet}:=!_{N}\left(\mathrm{~N}_{1}^{\bullet} \not 2 \cdots \not \mathrm{N}_{n}^{\bullet}\right) .
\end{array}
$$

We observe that the translation ${ }^{\bullet}$ inverts the polarity of the exponential formulas whereas it preserves the polarity of the other formulas. Given a multi-set of formulas $\Gamma=\mathrm{F}_{1}, \ldots, \mathrm{F}_{k}$ of $\mathbf{M E L L}_{\text {pol }}^{*}$ we write $\Gamma^{\bullet}$ for the multi-set $\mathrm{F}_{1}^{\bullet}, \ldots, \mathrm{F}_{k}^{\bullet}$ of formulas of MELLS.

Consider now a sequent $\vdash \Delta=\vdash \mathrm{N}_{1}, \ldots, \mathrm{N}_{k-1}, \mathrm{~F}_{k}$ of $\mathbf{M E L L}_{\mathrm{pol}}^{*}$. Since $\mathrm{N}_{1}, \ldots, \mathrm{N}_{k-1}$ are ?-formula, the multi-set $\mathrm{N}_{1}^{\bullet}, \ldots, \mathrm{N}_{k-1}^{\bullet}, \mathrm{F}_{k}^{\bullet}$ contains at most one occurrence of negative formula. Hence, every sequent $\vdash \Delta$ of MELL $_{\text {pol }}^{*}$ is sent to a sequent $\vdash \Delta^{\bullet}$ of MELLS.

We are now ready to show the converse correspondence.

Proposition A.13. If $\vdash \Delta$ is derivable in $\mathbf{M E L L}_{\mathrm{pol}}^{*}$ then $\vdash \Delta^{\bullet}$ is derivable in MELLS.

Proof. By induction on the height of a derivation $\pi$ of a sequent $\vdash \Delta$ in MELL $_{\text {pol }}^{*}$.

$\otimes_{n}$ : Suppose that $\pi$ ends with

$$
\frac{\vdash ? \Gamma_{1}, \mathrm{P}_{1} \quad \ldots \quad \vdash ? \Gamma_{n}, \mathrm{P}_{n}}{\vdash ? \Gamma_{1}, \ldots, ? \Gamma_{n}, \mathrm{P}_{1} \otimes \cdots \otimes \mathrm{P}_{n}} \otimes n
$$

We have to show a derivation of

$$
\vdash ? \Gamma_{1}, \ldots, ? \Gamma_{n}, \mathrm{P}_{1} \otimes \cdots \otimes \mathrm{P}_{n}^{\bullet}=\vdash ? \Gamma_{1}^{\bullet}, \ldots, ? \Gamma_{n}^{\bullet}, ?_{P}\left(\mathrm{P}_{1}^{\bullet} \otimes \cdots \otimes \mathrm{P}_{n}^{\bullet}\right) .
$$

By inductive hypothesis, the sequent $\vdash$ ? $\Gamma_{i}^{\bullet}, \mathrm{P}_{i}^{\bullet}$ is derivable for any $1 \leq i \leq n$. By Proposition A.2 (1) $\vdash ? \Gamma_{1}^{\bullet}, \ldots, ? \Gamma_{n}^{\bullet}, \mathrm{Q}^{\bullet}, \mathrm{P}_{i}^{\bullet}$ is also derivable for any $1 \leq i \leq n$, where $\mathrm{Q}^{\bullet}=?_{P}\left(\mathrm{P}_{1}^{\bullet} \otimes \cdots \otimes \mathrm{P}_{n}^{\bullet}\right)$. We take:

$$
\frac{\vdash ? \Gamma_{1}^{\bullet}, \ldots, ? \Gamma_{n}^{\bullet}, \mathrm{Q}^{\bullet}, \mathrm{P}_{1}^{\bullet} \quad \ldots \quad \vdash ? \Gamma_{1}^{\bullet}, \ldots, ? \Gamma_{n}^{\bullet}, \mathrm{Q}^{\bullet}, \mathrm{P}_{n}^{\bullet}}{\vdash ? \Gamma_{1}^{\bullet}, \ldots, ? \Gamma_{n}^{\bullet}, \mathrm{Q}^{\bullet}}
$$

$\gamma_{n}:$ Suppose that $\pi$ ends with

$$
\frac{\vdash ? \Gamma, \mathrm{N}_{1}, \ldots, \mathrm{N}_{n}}{\vdash ? \Gamma, \mathrm{N}_{1} 2 \gamma \ldots 2 \mathrm{~N}_{n}} \gamma_{n}
$$

We have to show a derivation of

$$
\vdash ? \Gamma, \mathrm{N}_{1} 28 \ldots 28 \mathrm{~N}_{n}^{\bullet}=\vdash ? \Gamma^{\bullet}, !_{N}\left(\mathrm{~N}_{1}^{\bullet} \not 2 \ldots 8 \mathrm{~N}_{n}^{\bullet}\right) \text {. }
$$

By inductive hypothesis, the sequent $\vdash ? \Gamma^{\bullet}, \mathrm{N}_{1}^{\bullet}, \ldots, \mathrm{N}_{n}^{\bullet}$ is derivable. We take:

$$
\frac{\vdash ? \Gamma^{\bullet}, \mathrm{N}_{1}^{\bullet}, \ldots, \mathrm{N}_{n}^{\bullet}}{\vdash ? \Gamma^{\bullet}, !_{N}\left(\mathrm{~N}_{1}^{\bullet} \not 2 \ldots \gamma \mathrm{N}_{n}^{\bullet}\right)} \mathrm{Neg}_{n}
$$

!: Suppose that $\pi$ ends with

$$
\frac{\vdash ? \Gamma, \mathrm{M}}{\vdash ? \Gamma, ! \mathrm{M}}
$$

By inductive hypothesis, the sequent $\vdash$ ? $\Gamma^{\bullet}, \mathrm{M}^{\bullet}$ is derivable. Since $! \mathrm{M} \bullet=\mathrm{M}^{\bullet}$, we conclude the argument.

?: Suppose that $\pi$ ends with 


$$
\frac{\vdash ? \Gamma, \mathrm{Q}}{\vdash ? \Gamma, ? \mathrm{Q}} ?
$$

By inductive hypothesis, the sequent $\vdash ?^{\bullet}, \mathrm{Q}^{\bullet}$ is derivable. Since $? \mathrm{Q}^{\bullet}=\mathrm{Q}^{\bullet}$, we conclude the argument.

W : Suppose that $\pi$ ends with

$$
\frac{\vdash \Delta}{\vdash \Delta, \mathrm{N}} \mathrm{W}
$$

By inductive hypothesis, the sequent $\vdash \Delta^{\bullet}$ is derivable. By Proposition A.2 (1), the sequent $\vdash \Delta^{\bullet}, N^{\bullet}$ is also derivable.

C : Suppose that $\pi$ ends with

$$
\frac{\vdash \Delta, \mathrm{N}, \mathrm{N}}{\vdash \Delta, \mathrm{N}} \mathrm{C}
$$

By inductive hypothesis, the sequent $\vdash \Delta^{\bullet}, N^{\bullet}, N^{\bullet}$ is derivable. By Proposition A.2 (2), the sequent $\vdash \Delta^{\bullet}, N^{\bullet}$ is also derivable.

Regarding the composition of the translations ${ }^{\circ}$ and $\bullet$, we observe the following properties.

\section{Lemma A.14.}

(1) For any formula $F$ of MELLS, we have $\left(F^{\circ}\right)^{\bullet}=F$.

(2) For any exponential formula $\mathrm{F}$ of $\mathbf{M E L L}_{\text {pol }}^{*}$, we have $\left(\mathrm{F}^{\bullet}\right)^{\circ}=\mathrm{F}$.

(3) For any sequent $\vdash \Gamma$ of MELLS, we have $\left(\vdash \Gamma^{\circ}\right)^{\bullet}=\vdash \Gamma$.

(4) Let $\Delta=\mathrm{F}_{1}, \ldots, \mathrm{F}_{n}$. We get $\left(\vdash \mathrm{F}_{1}, \ldots, \mathrm{F}_{n} \bullet\right)^{\circ}=\left(\vdash \mathrm{F}_{1}^{\bullet}, \ldots, \mathrm{F}_{n}^{\bullet}\right)^{\circ}=\vdash\left(\mathrm{F}_{1}^{\bullet}\right)^{\circ}, \ldots,\left(\mathrm{F}_{n}^{\bullet}\right)^{\circ}$, and by point (2) above we conclude the argument.

(5) For any sequent $\vdash \Delta$ of MELL $_{\text {pol }}^{*}$ consisting of exponential formulas only, we have $(\vdash \Delta \bullet)^{\circ}=\vdash \Delta$.

Proof.

(1) By induction on the depth of $F$. Let $P=?_{P}\left(N_{1} \otimes \cdots \otimes N_{n}\right)$. We have:

$$
\begin{aligned}
\left(P^{\circ}\right)^{\bullet} & =?_{P}\left(N_{1} \otimes \cdots \otimes N_{n}\right) \circ \bullet \\
& =?\left(N_{1}^{\circ} \otimes \cdots \otimes N_{n}^{\circ}\right) \\
& =N_{1}^{\circ} \otimes \cdots \otimes N_{n}^{\circ} \bullet \\
& =?_{P}\left(\left(N_{1}^{\circ} \bullet \otimes \cdots \otimes\left(N_{n}^{\circ}\right)^{\bullet}\right),\right.
\end{aligned}
$$

and by inductive hypothesis we conclude the argument. The negative case is similar.

(2) By induction on the depth of $\mathrm{F}$. Let $\mathrm{P}=!\left(\mathrm{N}_{1} \not \gamma \ldots \gamma \mathrm{N}_{n}\right)$. We have:

$$
\begin{aligned}
& \left(\mathrm{P}^{\bullet}\right)^{\circ}=!\left(\mathrm{N}_{1} \not 2 \ldots \gamma \mathrm{N}_{n}\right) \bullet \circ \\
& =\mathrm{N}_{1} \not 8 \ldots \gamma \mathrm{N}_{n} \bullet \\
& =!_{N}\left(\mathrm{~N}_{1}^{\bullet} \not \gamma \ldots \not \mathrm{N}_{n}^{\bullet}\right)^{\circ} \\
& =!\left(\left(\mathrm{N}_{1}^{\bullet}\right)^{\circ} \not 2 \ldots \not 8\left(\mathrm{~N}_{n}^{\bullet}\right)^{\circ}\right) \text {, }
\end{aligned}
$$

and by inductive hypothesis we conclude the argument. The negative case is similar.

(3) Let $\Gamma=F_{1}, \ldots, F_{n}$. We get $\left(\vdash F_{1}, \ldots, F_{n}^{\circ}\right)^{\bullet}=\left(\vdash F_{1}^{\circ}, \ldots, F_{n}^{\circ}\right)^{\bullet}=\vdash\left(F_{1}^{\circ}\right)^{\bullet}, \ldots,\left(F_{n}^{\circ}\right)^{\bullet}$, and by point (1) above we conclude the argument.

(4) Let $\Delta=\mathrm{F}_{1}, \ldots, \mathrm{F}_{n}$. We get $\left(\vdash \mathrm{F}_{1}, \ldots, \mathrm{F}_{n}^{\bullet}\right)^{\circ}=\left(\vdash \mathrm{F}_{1}^{\bullet}, \ldots, \mathrm{F}_{n}^{\bullet}\right)^{\circ}=\vdash\left(\mathrm{F}_{1}^{\bullet}\right)^{\circ}, \ldots,\left(\mathrm{F}_{n}^{\bullet}\right)^{\circ}$, and by point (2) above we conclude the argument.

We can finally collect the previous results in the next theorem. 
Theorem A.15.

(1) Let $\vdash \Delta$ be a sequent of $\mathbf{M E L L}_{\mathrm{pol}}$ consisting of exponential formulas only. $\vdash \Delta$ is derivable in $\mathbf{M E L L}_{\mathrm{pol}}$ if and only if $\vdash \Delta^{\bullet}$ is derivable in MELLS.

(2) $\vdash \Gamma$ is derivable in MELLS if and only if $\vdash \Gamma^{\circ}$ is derivable in $\mathbf{M E L L}_{\mathrm{pol}}$.

Proof.

(1) If $\vdash \Delta$ is a derivable sequent of $\mathbf{M E L L}_{\mathrm{pol}}$, then by Lemma A.9 it contains at most one occurrence of positive formula. Since $\vdash \Delta$ is made of exponential formulas only, it is of the form $\vdash$ ? $\Gamma$, $F$, where $F$ is either a ?-formula or a !-formula. In particular, $\vdash \Delta$ is a sequent of $\mathbf{M E L L}_{\text {pol }}^{*}$. By Proposition A.11, $\vdash \Delta$ is a derivable sequent of $\mathbf{M E L L}_{\text {pol }}^{*}$. By Proposition A.13, $\vdash \Delta^{\bullet}$ is derivable in MELLS.

Conversely, if $\vdash \Delta^{\bullet}$ is derivable in MELLS then, by Proposition A.12, $\left(\vdash \Delta^{\bullet}\right)^{\circ}$ is derivable in MELL $_{\text {pol }}^{*}$ and hence in of MELL $_{\text {pol }}$. By Lemma A.14 (4), $\left(\vdash \Delta^{\bullet}\right)^{\circ}=\vdash \Delta$ and we conclude the argument.

(2) If $\vdash \Gamma$ is derivable in MELLS then, by Proposition A.12, $\vdash \Gamma^{\circ}$ is derivable in $\mathbf{M E L L}_{\text {pol }}^{*}$ and hence also in MELL $_{\text {pol }}$.

For the converse, we observe that by the definition of ${ }^{\circ}, \vdash \Gamma^{\circ}$ is a sequent of $\mathbf{M E L L}_{\text {pol }}^{*}$. Since we are assuming that it is derivable in $\mathbf{M E L L}_{\mathrm{pol}}$, by Proposition A.11, it is also derivable in MELL $_{\text {pol }}^{*}$. By Proposition A.13, $\left(\vdash \Gamma^{\circ}\right)^{\bullet}$ is derivable in MELLS. By Lemma A.14 $(3),\left(\vdash \Gamma^{\circ}\right)^{\bullet}=\vdash \Gamma$ and we conclude the argument.

\section{Appendix B. Soundness}

We first show some technical lemmas.

Lemma B.1 (Logical rules).

(1) Let $\mathbf{P}=\mathbf{F}^{+}\left(\mathbf{N}_{1}, \ldots, \mathbf{N}_{n}\right)$ be a positive behaviour. If $\mathcal{E}_{1} \in \vdash \mathbf{\Pi}, \mathbf{N}_{\sigma 1}, \ldots, \mathcal{E}_{n} \in \vdash \boldsymbol{\Pi}, \mathbf{N}_{\sigma n}$ then $\mathcal{D}:=\left(\sigma, I_{n}\right)^{+} .\left\{\mathcal{E}_{1}, \ldots, \mathcal{E}_{n}\right\} \in \vdash \boldsymbol{\Pi}, \mathbf{P}_{\sigma}$. Moreover, if $\mathcal{E}_{1}, \ldots, \mathcal{E}_{n}$ are winning, then $\mathcal{D}$ is winning.

(2) Let $\mathbf{N}=\mathbf{F}^{-}\left(\mathbf{P}_{1}, \ldots, \mathbf{P}_{n}\right)$ be a negative behaviour.

If $\mathcal{E} \in \vdash \boldsymbol{\Pi}, \mathbf{P}_{\xi 1}, \ldots, \mathbf{P}_{\xi n}$ then $\mathcal{D}:=\left(\xi, I_{n}\right)^{-} . \mathcal{E} \in \vdash \mathbf{\Pi}, \mathbf{N}_{\xi}$.

Moreover, if $\mathcal{E}$ is winning, then $\mathcal{D}$ is winning.

Proof. Let us fix $\boldsymbol{\Pi}=\mathbf{Q}_{\alpha_{1}}, \ldots, \mathbf{Q}_{\alpha_{k}}$ and arbitrary strategies $\mathcal{F}_{1} \in \mathbf{Q}_{\alpha_{1}}^{\perp}, \ldots, \mathcal{F}_{k} \in \mathbf{Q}_{\alpha_{k}}^{\perp}$. We will write $\left(\mathcal{F}_{j}\right)$ and $\left(\mathcal{F}_{j}\right) \in \mathbf{C}$ for $\mathcal{F}_{1}, \ldots, \mathcal{F}_{k}$ and $\mathcal{F}_{1} \in \mathbf{Q}_{\alpha_{1}}^{\perp}, \ldots, \mathcal{F}_{k} \in \mathbf{Q}_{\alpha_{k}}^{\perp}$ respectively.

(1) For sake of clarity, we distinguish two subcases.

If $n=0$, then $\mathbf{P}=?_{P} \mathbf{1}$ and $\mathcal{D}$ is just $(\sigma, \emptyset)^{+}$. It is then immediate to show (1), since for any $\mathcal{A} \in \mathbf{P}_{\sigma}^{\perp}=!_{N} \perp_{\sigma}$, we have $\llbracket \mathcal{D}, \mathcal{A},\left(\mathcal{F}_{j}\right) \rrbracket=\llbracket \mathcal{D}, \mathcal{A} \rrbracket$ and $\mathcal{D}$ and $\mathcal{A}$ are obviously orthogonal. It is also immediate to check that $\mathcal{D}$ is winning.

If $n>0$, by Proposition 10.6, $\mathcal{E}_{i} \in \vdash \mathbf{\Pi}, \mathbf{N}_{\sigma i}$ if and only if $\mathcal{E}_{i}^{\prime}:=\llbracket \mathcal{E}_{i},\left(\mathcal{F}_{j}\right) \rrbracket \in \mathbf{N}_{\sigma i}$, for any $1 \leq i \leq n$. By construction of $\mathbf{F}^{+}\left(\mathbf{N}_{1}, \ldots, \mathbf{N}_{n}\right)$, the strategy $\left(\sigma, I_{n}\right)^{+} .\left\{\mathcal{E}_{1}^{\prime}, \ldots, \mathcal{E}_{n}^{\prime}\right\} \in$ $\mathbf{P}_{\sigma}$. By using Lemma 10.2 and Proposition 10.6 again, we conclude $\mathcal{D} \in \vdash \boldsymbol{\Pi}, \mathbf{P}_{\sigma}$.

As for winning conditions, the only one which is not immediate is materiality. Let $\mathcal{A}$ be a strategy in $\mathbf{N}_{\sigma i}^{\perp}$ and $\mathcal{A}^{*}:=\left(\sigma, I_{n}\right)^{-} . \mathcal{A} \in \mathbf{P}_{\sigma}^{\perp}$. Observe that by construction $\left(\sigma, I_{n}\right)^{+}$ occurs linearly in $\mathcal{D}$ at the root. This makes the interaction of the cut-net $\left\{\mathcal{D}, \mathcal{A}^{*},\left(\mathcal{F}_{j}\right)\right\}$ particularly simple to describe: (i) $\left(\sigma, I_{n}\right)^{+}$and $\left(\sigma, I_{n}\right)^{-}$match, (ii) after matching, the 
interaction is exactly as in the cut-net $\left\{\mathcal{E}_{i}, \mathcal{A},\left(\mathcal{F}_{j}\right)\right\}$. So we have (in the notation of Definition 11.2)

$$
\mathcal{D}\left[\mathcal{A}^{*},\left(\mathcal{F}_{j}\right)\right]=\left(\sigma, I_{n}\right)^{+} . \mathcal{E}_{i}\left[\mathcal{A},\left(\mathcal{F}_{j}\right)\right] .
$$

Since $\mathcal{E}_{i}$ is material, if we let vary $\mathcal{A} \in \mathbf{N}_{\sigma i}^{\perp}$ and $\left(\mathcal{F}_{j}\right) \in \mathbf{C}$ and use the counter-strategies $\mathcal{A}^{*},\left(\mathcal{F}_{j}\right)$, we can visit the whole subtree $\left(\sigma, I_{n}\right)^{+} . \mathcal{E}_{i} \subseteq \mathcal{D}$. Applying the same reasoning to each $1 \leq i \leq n$ we have our claim.

(2) By Proposition 10.6, $\mathcal{E} \in \vdash \boldsymbol{\Pi}, \mathbf{P}_{\xi 1}, \ldots, \mathbf{P}_{\xi n}$ if and only if $\mathcal{E}^{\prime}:=\llbracket \mathcal{E},\left(\mathcal{F}_{j}\right) \rrbracket \in \vdash \mathbf{P}_{\xi 1}, \ldots$, $\mathbf{P}_{\xi n}$. By Proposition 10.8, $\left(\xi, I_{n}\right)^{-} \cdot \mathcal{E}^{\prime} \in \vdash \mathbf{N}_{\xi}$. Since we have that $\left(\xi, I_{n}\right)^{-} \cdot \llbracket \mathcal{E},\left(\mathcal{F}_{j}\right) \rrbracket=$ $\llbracket\left(\xi, I_{n}\right)^{-} \cdot \mathcal{E},\left(\mathcal{F}_{j}\right) \rrbracket$, by using Proposition 10.6 again, we conclude $\mathcal{D} \in \vdash \mathbf{\Pi}, \mathbf{N}_{\xi}$.

As for winning conditions, the only one which is not immediate is materiality. For $\mathcal{A}_{1}, \in \mathbf{P}_{\xi 1}^{\perp}, \ldots, \mathcal{A}_{n} \in \mathbf{P}_{\xi n}^{\perp}$, let us set $\mathcal{A}^{*}:=\left(\xi, I_{n}\right)^{+} .\left\{\mathcal{A}_{1}, \ldots, \mathcal{A}_{n}\right\} \in \mathbf{N}_{\xi}^{\perp}$. Since by construction $\left(\xi, I_{n}\right)^{+}$occurs linearly in $\mathcal{A}^{*}$, the interaction of the cut-net $\left\{\mathcal{D}, \mathcal{A}^{*},\left(\mathcal{F}_{j}\right)\right\}$ can be simply described as follows: (i) the actions $\left(\xi, I_{n}\right)^{+}$and $\left(\xi, I_{n}\right)^{-}$match, (ii) after matching the interaction is the exactly as in the cut-net $\left\{\mathcal{E}, \mathcal{A}_{1}, \ldots, \mathcal{A}_{n},\left(\mathcal{F}_{j}\right)\right\}$. So we have

$$
\mathcal{D}\left[\mathcal{A}^{*},\left(\mathcal{F}_{j}\right)\right]=\left(\xi, I_{n}\right)^{-} \cdot \mathcal{E}\left[\mathcal{A}_{1}, \ldots, \mathcal{A}_{n},\left(\mathcal{F}_{j}\right)\right] .
$$

Since $\mathcal{E}$ is material, if we let vary $\mathcal{A}_{1}, \in \mathbf{P}_{\xi 1}^{\perp}, \ldots, \mathcal{A}_{n} \in \mathbf{P}_{\xi n}^{\perp}$ and $\left(\mathcal{F}_{j}\right) \in \mathbf{C}$, we can use the counter-strategies $\mathcal{A}^{*},\left(\mathcal{F}_{j}\right)$ in order to completely visit $\mathcal{D}$.

Lemma B.2 (Structural rules).

(1) Weakening: if $\mathcal{D} \in \vdash \boldsymbol{\Gamma}$ then $\mathcal{D} \in \vdash \boldsymbol{\Gamma}, \mathbf{Q}_{\xi}$.

Moreover, if $\mathcal{D}$ is winning (in $\vdash \mathbf{\Gamma}$ ) then $\mathcal{D}$ is winning (in $\left.\vdash \boldsymbol{\Gamma}, \mathbf{Q}_{\xi}\right)$.

(2) Contraction: if $\mathcal{D} \in \vdash \boldsymbol{\Gamma}, \mathbf{Q}_{\xi}, \mathbf{Q}_{\sigma}$ then $\mathcal{D}[\xi / \sigma] \in \vdash \boldsymbol{\Gamma}, \mathbf{Q}_{\xi}$.

Moreover, if $\mathcal{D}$ is winning then $\mathcal{D}[\xi / \sigma]$ is winning.

Proof. Let us fix $\boldsymbol{\Gamma}=\mathbf{F}_{\alpha_{1}}, \ldots, \mathbf{F}_{\alpha_{k}}$ and arbitrary strategies $\mathcal{F}_{1} \in \mathbf{F}_{\alpha_{1}}^{\perp}, \ldots, \mathcal{F}_{k} \in \mathbf{F}_{\alpha_{k}}^{\perp}$. We will write $\left(\mathcal{F}_{j}\right)$ and $\left(\mathcal{F}_{j}\right) \in \mathbf{C}$ for $\mathcal{F}_{1}, \ldots, \mathcal{F}_{k}$ and $\mathcal{F}_{1} \in \mathbf{F}_{\alpha_{1}}^{\perp}, \ldots, \mathcal{F}_{k} \in \mathbf{F}_{\alpha_{k}}^{\perp}$ respectively.

(1) Since no action with name $\xi$ occurs in $\mathcal{D}$, we have for any $\mathcal{E} \in \mathbf{Q}_{\xi}^{\perp}$ :

$$
\llbracket \mathcal{D},\left(\mathcal{F}_{j}\right) \rrbracket=\llbracket \mathcal{D}, \mathcal{E},\left(\mathcal{F}_{j}\right) \rrbracket,
$$

where $\mathcal{D}$ is taken on the interface $\alpha_{1}, \ldots, \alpha_{k}$ of $\vdash \boldsymbol{\Gamma}\left(\operatorname{resp} . \alpha_{1}, \ldots, \alpha_{k}, \xi^{+}\right.$of $\left.\vdash \boldsymbol{\Gamma}, \mathbf{Q}_{\xi}\right)$ on the LHS (resp. RHS) of the equality above. Moreover it is easily seen that the interactions of the two cut-nets are exactly the same. The result then immediately follows.

(2) We first prove that $\mathcal{D} \in \vdash \boldsymbol{\Gamma}, \mathbf{Q}_{\xi}, \mathbf{Q}_{\sigma}$ implies $\mathcal{D}[\xi / \sigma] \in \vdash \boldsymbol{\Gamma}, \mathbf{Q}_{\xi}$. By hypothesis, for any $\mathcal{A}, \mathcal{B} \in \mathbf{Q}_{\xi}^{\perp}$, we have that $\mathcal{D} \perp\left\{\mathcal{A}, \mathcal{B}[\sigma / \xi],\left(\mathcal{F}_{j}\right)\right\}$. When $\mathcal{A}=\mathcal{B}$, we have that $\mathcal{D} \perp\left\{\mathcal{A}, \mathcal{A}[\sigma / \xi],\left(\mathcal{F}_{j}\right)\right\}$ which implies $\mathcal{D}[\xi / \sigma] \perp\left\{\mathcal{A},\left(\mathcal{F}_{j}\right)\right\}$ by Proposition 8.14. Hence, we have $\mathcal{D}[\xi / \sigma] \in \vdash \boldsymbol{\Gamma}, \mathbf{Q}_{\xi}$.

As for winning conditions, the only one which is not immediate to prove is materiality. By Lemma 10.10 (2), we have that $\mathbf{Q}_{\xi}=\left(\mathbf{Q}_{\xi}^{\perp}\langle x\rangle\right)^{\perp}$, where recall that $\mathbf{Q}_{\xi}^{\perp}\langle x\rangle$ consists of the negative strategies of $\mathbf{Q}^{\perp}$ which have a unique negative root $x=\left(\xi, I_{n}\right)$. Since $\mathcal{D}$ is material in $\vdash \boldsymbol{\Gamma}, \mathbf{Q}_{\xi}, \mathbf{Q}_{\sigma}$, we have by Definition 11.5.

$$
\mathcal{D}=\bigcup\left\{\mathcal{D}\left[\mathcal{A}, \mathcal{B}[\sigma / \xi],\left(\mathcal{F}_{j}\right)\right]: \mathcal{A}, \mathcal{B} \in \mathbf{Q}_{\xi}^{\perp}\langle x\rangle \text { and }\left(\mathcal{F}_{j}\right) \in \mathbf{C}\right\} .
$$

Since $\mathcal{A}, \mathcal{B}$ have the same root $x$, by Lemma 10.10 (1) the strategy $\mathcal{A}+{ }^{\tau} \mathcal{B} \in \mathbf{Q}_{\xi}^{\perp}$. The strategy $\mathcal{A}+{ }^{\tau} \mathcal{B}$ has the same unique root $x$ too, hence $\mathcal{A}+{ }^{\tau} \mathcal{B} \in \mathbf{Q}_{\xi}^{\perp}\langle x\rangle$. 
Observe that using the counter-strategy $\mathcal{A}+{ }^{\tau} \mathcal{B}$ we are able to visit the part of $\mathcal{D}$ which can be visited interchanging $\mathcal{A}$ and $\mathcal{B}$ (and possibly visit new actions), i.e.,

$$
\begin{array}{lll}
\mathcal{D}\left[\mathcal{A}, \mathcal{B}[\sigma / \xi],\left(\mathcal{F}_{j}\right)\right] & \subseteq \mathcal{D}\left[\mathcal{A}+{ }^{\tau} \mathcal{B},\left(\mathcal{A}+{ }^{\tau} \mathcal{B}\right)[\sigma / \xi],\left(\mathcal{F}_{j}\right)\right] \\
\mathcal{D}\left[\mathcal{B}, \mathcal{A}[\sigma / \xi],\left(\mathcal{F}_{j}\right)\right] & \subseteq & \mathcal{D}\left[\mathcal{A}+{ }^{\tau} \mathcal{B},\left(\mathcal{A}+{ }^{\tau} \mathcal{B}\right)[\sigma / \xi],\left(\mathcal{F}_{j}\right)\right]
\end{array}
$$

But then, we have that

$$
\begin{aligned}
\mathcal{D} & =\bigcup\left\{\mathcal{D}\left[\mathcal{A}+{ }^{\tau} \mathcal{B},\left(\mathcal{A}+{ }^{\tau} \mathcal{B}\right)[\sigma / \xi],\left(\mathcal{F}_{j}\right)\right]: \mathcal{A}+{ }^{\tau} \mathcal{B} \in \mathbf{Q}_{\xi}^{\perp}\langle x\rangle \text { and }\left(\mathcal{F}_{j}\right) \in \mathbf{C}\right\} \\
& =\bigcup\left\{\mathcal{D}\left[\mathcal{C}, \mathcal{C}[\sigma / \xi],\left(\mathcal{F}_{j}\right)\right]: \mathcal{C} \in \mathbf{Q}_{\xi}^{\perp}\langle x\rangle \text { and }\left(\mathcal{F}_{j}\right) \in \mathbf{C}\right\} .
\end{aligned}
$$

Similarly as in Proposition 8.14, we can derive that

$$
\mathcal{D}\left[\mathcal{C}, \mathcal{C}[\sigma / \xi],\left(\mathcal{F}_{j}\right)\right][\xi / \sigma]=\mathcal{D}[\xi / \sigma]\left[\mathcal{C},\left(\mathcal{F}_{j}\right)\right]
$$

because the interactions of the cut-nets $\left\{\mathcal{D}, \mathcal{C}, \mathcal{C}[\sigma / \xi],\left(\mathcal{F}_{j}\right)\right\}$ and $\left\{\mathcal{D}[\xi / \sigma], \mathcal{C},\left(\mathcal{F}_{j}\right)\right\}$ differ only in the names of some hidden actions but have the same pointer structure. Hence:

$$
\begin{aligned}
\mathcal{D}[\xi / \sigma] & =\bigcup\left\{\mathcal{D}\left[\mathcal{C}, \mathcal{C}[\sigma / \xi],\left(\mathcal{F}_{j}\right)\right]: \mathcal{C} \in \mathbf{Q}_{\xi}^{\perp}\langle x\rangle \text { and }\left(\mathcal{F}_{j}\right) \in \mathbf{C}\right\}[\xi / \sigma] \\
& =\bigcup\left\{\mathcal{D}\left[\mathcal{C}, \mathcal{C}[\sigma / \xi],\left(\mathcal{F}_{j}\right)\right][\xi / \sigma]: \mathcal{C} \in \mathbf{Q}_{\xi}^{\perp}\langle x\rangle \text { and }\left(\mathcal{F}_{j}\right) \in \mathbf{C}\right\} \\
& =\bigcup\left\{\mathcal{D}[\xi / \sigma]\left[\mathcal{C},\left(\mathcal{F}_{j}\right)\right]: \mathcal{C} \in \mathbf{Q}_{\xi}^{\perp}\langle x\rangle \text { and }\left(\mathcal{F}_{j}\right) \in \mathbf{C}\right\},
\end{aligned}
$$

which shows the materiality of $\mathcal{D}[\xi / \sigma]$ in $\vdash \boldsymbol{\Gamma}, \mathbf{Q}_{\xi}$.

We can now prove the following:

Proposition B.3. Let $\pi$ be a cut-free derivation of a sequent $\vdash \Gamma$ in MELLS and $\mathcal{D}(\pi)$ be the interpretation of $\pi$ in a sequent of behaviours $\vdash \Gamma . \mathcal{D}(\pi)$ is a winning strategy in $\vdash \boldsymbol{\Gamma}$.

Proof. By induction on the height of $\pi$. As for positive rules, we use Lemmas B.1 (1) and B.2. As for negatives rules, we use Lemma B.1 (2).

In order to expand the previous proposition to derivation with cuts, we need to study the relation between composition of strategies and the procedure of cut-elimination defined in the proof of admissibility of the cut-rule of MELLS (Theorem A.3).

We first show the following:

Lemma B.4 (Cut-rule). If $\mathcal{D} \in \vdash \boldsymbol{\Xi}, \boldsymbol{\Pi}, \mathbf{P}_{\xi}$ and $\mathcal{E} \in \vdash \boldsymbol{\Delta}, \mathbf{P}_{\xi}^{\perp}$ then $\llbracket \mathcal{D}, \mathcal{E} \rrbracket \in \vdash \boldsymbol{\Xi}, \boldsymbol{\Pi}, \boldsymbol{\Delta}$

Proof. We only consider the case in which $\boldsymbol{\Xi}$ is empty, the case $\boldsymbol{\Xi}=\mathbf{N}$ is similar.

Let $\boldsymbol{\Pi}=\mathbf{A}_{\alpha_{1}}, \ldots, \mathbf{A}_{\alpha_{n}}$ and $\boldsymbol{\Delta}=\mathbf{B}_{\beta_{1}}, \ldots, \mathbf{B}_{\beta_{k}}$ on disjoint interfaces $\alpha_{1}^{+}, \ldots, \alpha_{n}^{+}$and $\beta_{1}^{+}, \ldots, \beta_{k}^{+}$respectively. Let $\mathcal{A}_{1} \in \mathbf{A}_{\alpha_{1}}^{\perp}, \ldots, \mathcal{A}_{n} \in \mathbf{A}_{\alpha_{n}}^{\perp}$ and $\mathcal{B}_{1} \in \mathbf{B}_{\beta_{1}}^{\perp}, \ldots, \mathcal{B}_{k} \in \mathbf{B}_{\beta_{k}}^{\perp}$ be arbitrary strategies and write $\left(\mathcal{A}_{j}\right)$ and $\left(\mathcal{B}_{l}\right)$ for $\mathcal{A}_{1}, \ldots, \mathcal{A}_{n}$ and $\mathcal{B}_{1}, \ldots, \mathcal{B}_{k}$ respectively.

By Proposition 10.6 we have that $\llbracket \mathcal{D},\left(\mathcal{A}_{j}\right) \rrbracket \in \mathbf{P}_{\xi}$ and $\llbracket \mathcal{E},\left(\mathcal{B}_{l}\right) \rrbracket \in \mathbf{P}_{\xi}^{\perp}$ which implies that $\llbracket \llbracket \mathcal{D},\left(\mathcal{A}_{j}\right) \rrbracket, \llbracket \mathcal{E},\left(\mathcal{B}_{l}\right) \rrbracket \rrbracket$ is total. By associativity, we have that $\llbracket \llbracket \mathcal{D}, \mathcal{E} \rrbracket,\left(\mathcal{A}_{j}\right),\left(\mathcal{B}_{l}\right) \rrbracket$ is total, which shows that $\llbracket \mathcal{D}, \mathcal{E} \rrbracket \in \vdash \boldsymbol{\Pi}, \boldsymbol{\Delta}$.

We now relate our interpretation and Proposition A.2, which deals with the structural rules of MELLS. 


\section{Lemma B.5.}

(1) Let $\mathcal{D}(\pi)$ be the interpretation of a cut-free derivation $\pi$ of $\vdash \Gamma$ in a sequent of behaviours $\vdash \Gamma$ and $\mathcal{D}\left(\pi^{\prime}\right)$ be the interpretation of the cut-free derivation $\pi^{\prime}$ of $\vdash \Gamma, Q$ in the sequent of behaviours $\vdash \boldsymbol{\Gamma}, \mathbf{Q}_{\xi}$ as given by Proposition $\mathrm{A.2}(1)$. We have $\mathcal{D}(\pi)=\mathcal{D}\left(\pi^{\prime}\right)$.

(2) Let $\mathcal{D}(\pi)$ be interpretation of a cut-free derivation $\pi$ of $\vdash \Gamma, Q, Q$ in a sequent of behaviours $\vdash \boldsymbol{\Gamma}, \mathbf{Q}_{\xi}, \mathbf{Q}_{\sigma}$ and $\mathcal{D}\left(\pi^{\prime}\right)$ be the interpretation of the cut-free derivation $\pi^{\prime}$ of $\vdash \Gamma, Q$ in a sequent of behaviours $\vdash \boldsymbol{\Gamma}, \mathbf{Q}_{\xi}$ as given by Proposition A.2 (2). We have $\mathcal{D}\left(\pi^{\prime}\right)=\mathcal{D}(\pi)[\xi / \sigma]$.

Proof. By induction on the height of $\pi$.

We can finally show the correspondence between composition and cut-elimination.

Lemma B.6 (Composition and cut-elimination). Let $\pi$ and $\rho$ be cut-free derivations in MELLS of $\vdash \Xi, \Pi, P$ and $\vdash \Delta, P^{\perp}$ respectively and $\mathcal{D}(\pi)$ and $\mathcal{D}(\rho)$ be the interpretation of $\pi$ and $\rho$ in $\vdash \boldsymbol{\Xi}, \boldsymbol{\Pi}, \mathbf{P}_{\xi}$ and $\vdash \boldsymbol{\Delta}, \mathbf{P}_{\xi}^{\perp}$ respectively. Let $\theta$ be the cut-free derivation of $\vdash \Xi, \Pi, \Delta$ as given by Theorem $A .3$ and $\mathcal{D}(\theta)$ its interpretation in $\vdash \boldsymbol{\Xi}, \boldsymbol{\Pi}, \boldsymbol{\Delta}$.

We have $\llbracket \mathcal{D}(\pi), \mathcal{D}(\rho) \rrbracket=\mathcal{D}(\theta)$.

Proof. As in Theorem A.3, the proof is given by induction on the pair $(d(P), h(\pi)+h(\rho))$, where $d$ (resp. $h$ ) denotes the depth of a formula (resp. the height of a proof), as given in Definition A.1. We distinguish three subcases.

(a) The last rule of $\pi$ is a positive rule $\operatorname{Pos}_{n}$ and $P$ is principal in $\operatorname{Pos}_{n}$. As in Theorem A.3. we only consider the cases $n=0$ and $n=2$.

If $n=0$ and we have

$$
\begin{gathered}
\vdots \rho_{0} \\
\frac{\vdash \Delta}{\vdash \Pi, ?_{P} \mid} \operatorname{Pos}_{0} \quad \frac{\vdash \Delta, !_{N} \perp}{} \mathrm{Neg}_{0}
\end{gathered}
$$

the procedure described in the proof of Theorem A.3 gives the cut free derivation $\theta$ of $\vdash \Pi, \Delta$, where $\theta$ is obtained from $\rho_{0}$ by means of Proposition A.2 (1) (weakening on positive formulas).

By our interpretation, we have that $\mathcal{D}(\pi)=(\xi, \emptyset)^{+}$and $\mathcal{D}(\rho)=(\xi, \emptyset)^{-} \cdot \mathcal{D}\left(\rho_{0}\right)$. By normalization and Lemma B.5 $(1)$, we have $\llbracket \mathcal{D}(\pi), \mathcal{D}(\rho) \rrbracket=\mathcal{D}\left(\rho_{0}\right)=\mathcal{D}(\theta)$.

If $n=2$, for $P=?_{P}\left(N_{1} \otimes N_{2}\right)$ and $P^{\perp}=!_{N}\left(N_{1}^{\perp} \not \gamma N_{2}^{\perp}\right)$, we have

$$
\begin{array}{ccc}
\begin{array}{c}
\vdots \pi_{1} \\
\vdash \Pi, P, N_{1}
\end{array} & \vdots \pi_{2} & \vdots \rho_{0} \\
\vdash \Pi, P, N_{2} & \text { Pos }_{2} & \frac{\vdash \Delta, N_{1}^{\perp}, N_{2}^{\perp}}{\vdash \Delta, P^{\perp}} \text { Neg }_{2}
\end{array}
$$

Suppose that $\pi$ is interpreted by $\mathcal{D}(\pi)=(\sigma,\{1,2\})^{+} \cdot\left\{\mathcal{D}\left(\pi_{1}\right), \mathcal{D}\left(\pi_{2}\right)\right\}[\xi / \sigma]$ in the sequent of behaviours $\vdash \boldsymbol{\Pi}, \mathbf{P}$ on interface $\Pi, \xi^{+}$and $\rho$ is interpreted by $\mathcal{D}(\rho)=(\xi,\{1,2\})^{-} \cdot \mathcal{D}\left(\rho_{0}\right)$ in the sequent of behaviours $\vdash \boldsymbol{\Delta}, \mathbf{P}^{\perp}$ on interface $\Delta, \xi^{-}$. Since the construction of the cut-free derivation $\theta$ of $\vdash \Pi, \Delta$ (as given in Theorem A.3) involves "copies" of derivations and contractions, we also consider:

- $\mathcal{A}_{1}:=\mathcal{D}\left(\pi_{1}\right)\left[\Pi^{\prime} / \Pi\right]$, the interpretation of $\pi_{1}$ in the sequent of behaviours $\vdash \mathbf{\Pi}, \mathbf{P}, \mathbf{N}_{1}$ on interface $\Pi^{\prime}, \xi^{+}, \sigma_{1}^{-}$, 
- $\mathcal{A}_{2}:=\mathcal{D}\left(\pi_{2}\right)\left[\Pi^{\prime \prime} / \Pi\right]$, the interpretation of $\pi_{2}$ in the sequent of behaviours $\vdash \mathbf{\Pi}, \mathbf{P}, \mathbf{N}_{2}$ on interface $\Pi^{\prime \prime}, \xi^{+}, \sigma_{2}^{-}$,

- $\mathcal{B}:=\mathcal{D}(\rho)\left[\sigma / \xi, \Delta^{\prime} / \Delta\right]$, the interpretation of $\rho$ in the sequent of behaviours $\vdash \boldsymbol{\Delta}, \mathbf{P}^{\perp}$ on interface $\Delta^{\prime}, \sigma^{-}$,

- $\mathcal{B}_{0}:=\mathcal{D}\left(\rho_{0}\right)\left[\sigma 1 / \xi 1, \sigma 2 / \xi 2, \Delta^{\prime} / \Delta\right]$, the interpretation of $\rho_{0}$ in the sequent of behaviours $\vdash \boldsymbol{\Delta}, \mathbf{N}_{1}^{\perp}, \mathbf{N}_{2}^{\perp}$ on interface $\Delta^{\prime}, \sigma 1^{-}, \sigma 2^{-}$. Equivalently, $\mathcal{B}_{0}$ is obtained from $\mathcal{B}$ by removing its root $(\sigma,\{1,2\})^{-}$, i.e., $\mathcal{B}=(\sigma,\{1,2\})^{-}$. $\mathcal{B}_{0}$,

- $\mathcal{C}_{1}:=\mathcal{D}(\rho)\left[\Delta^{\prime \prime} / \Delta\right]$, the interpretation of $\rho$ in the sequent of behaviours $\vdash \boldsymbol{\Delta}, \mathbf{P}^{\perp}$ on interface $\Delta^{\prime \prime}, \xi^{-}$,

- $\mathcal{C}_{2}:=\mathcal{D}(\rho)\left[\Delta^{\prime \prime \prime} / \Delta\right]$, the interpretation of $\rho$ in the sequent of behaviours $\vdash \boldsymbol{\Delta}, \mathbf{P}^{\perp}$ on interface $\Delta^{\prime \prime \prime}, \xi^{-}$,

where the names $\Pi^{\prime}, \Pi^{\prime \prime}, \Delta^{\prime}, \Delta^{\prime \prime}, \Delta^{\prime \prime \prime}$ are all fresh and disjoint.

Recall that the procedure described in Theorem A.3 introduces cut-free derivations $\psi_{1}$ of $\vdash \Pi, \Delta, N_{1}$ (from $\pi_{1}$ and $\rho$ ), $\psi_{2}$ of $\vdash \Pi, \Delta, N_{2}$ (from $\pi_{2}$ and $\rho$ ), $\theta_{1}$ of $\vdash \Pi, \Delta, \Delta, N_{2}^{\perp}$ (from $\rho_{0}$ and $\psi_{1}$ ), $\theta_{2}$ of $\vdash \Pi, \Pi, \Delta, \Delta, \Delta$ (from $\theta_{1}$ and $\psi_{2}$ ). Finally, the cut-free derivation $\theta$ of $\vdash \Pi, \Delta$ is obtained from $\theta_{2}$ by means of contractions, in the sense of Proposition A.2 (2) (contraction on positive formulas).

In terms of our interpretation, we have:

$$
\mathcal{D}(\theta)=\llbracket \llbracket \mathcal{B}_{0}, \llbracket \mathcal{A}_{1}, \mathcal{C}_{1} \rrbracket \rrbracket, \llbracket \mathcal{A}_{2}, \mathcal{C}_{2} \rrbracket \rrbracket\left[\Pi / \Pi^{\prime}, \Pi / \Pi^{\prime \prime}, \Delta / \Delta^{\prime}, \Delta / \Delta^{\prime \prime}, \Delta / \Delta^{\prime \prime \prime}\right]
$$

where $\llbracket \mathcal{A}_{1}, \mathcal{C}_{1} \rrbracket\left(\right.$ resp. $\left.\llbracket \mathcal{A}_{2}, \mathcal{C}_{2} \rrbracket\right)$ interprets the derivation $\psi_{1}$ (resp. $\left.\psi_{2}\right), \llbracket \mathcal{B}_{0}, \llbracket \mathcal{A}_{1}, \mathcal{C}_{1} \rrbracket \rrbracket$ interprets the derivation $\theta_{1}, \llbracket \llbracket \mathcal{B}_{0}, \llbracket \mathcal{A}_{1}, \mathcal{C}_{1} \rrbracket \rrbracket, \llbracket \mathcal{A}_{2}, \mathcal{C}_{2} \rrbracket \rrbracket$ interprets the derivation $\theta_{2}$ and finally, by Lemma B.5 (2), the renamings $\left[\Pi / \Pi^{\prime}, \Pi / \Pi^{\prime \prime}, \Delta / \Delta^{\prime}, \Delta / \Delta^{\prime \prime}, \Delta / \Delta^{\prime \prime \prime}\right]$ take care of the contractions.

We have to show that $\llbracket \mathcal{D}(\pi), \mathcal{D}(\rho) \rrbracket=\mathcal{D}(\theta)$.

Writing $a$ (resp. $b$ ) for $(\sigma,\{1,2\})$ (resp. $(\xi,\{1,2\})$ and using Proposition 8.14 and the associativity of the normalization, we get:

$$
\begin{aligned}
\llbracket \mathcal{D}(\pi), \mathcal{D}(\rho) \rrbracket & =\llbracket a^{+} \cdot\left\{\mathcal{D}\left(\pi_{1}\right), \mathcal{D}\left(\pi_{2}\right)\right\}[\xi / \sigma], b^{-} \cdot \mathcal{D}\left(\rho_{0}\right) \rrbracket \\
& =\llbracket a^{+} \cdot\left\{\mathcal{D}\left(\pi_{1}\right), \mathcal{D}\left(\pi_{2}\right)\right\}, b^{-} \cdot \mathcal{D}\left(\rho_{0}\right), \mathcal{B} \rrbracket\left[\Delta / \Delta^{\prime}\right] \\
& =\llbracket \llbracket a^{+} \cdot\left\{\mathcal{D}\left(\pi_{1}\right), \mathcal{D}\left(\pi_{2}\right)\right\}, b^{-} \cdot \mathcal{D}\left(\rho_{0}\right) \rrbracket, \mathcal{B} \rrbracket\left[\Delta / \Delta^{\prime}\right]
\end{aligned}
$$

In $\llbracket a^{+} .\left\{\mathcal{D}\left(\pi_{1}\right), \mathcal{D}\left(\pi_{2}\right)\right\}, b^{-} \cdot \mathcal{D}\left(\rho_{0}\right) \rrbracket$, the action $a^{+}$is visible and then we can "push" $\mathcal{D}(\rho)$ in both premises $\mathcal{D}\left(\pi_{1}\right)$ and $\mathcal{D}\left(\pi_{2}\right)$ using the strategies $\mathcal{A}_{1}, \mathcal{A}_{2}, \mathcal{C}_{1}, \mathcal{C}_{2}$ introduced above as follows:

$$
\llbracket a^{+} \cdot\left\{\mathcal{D}\left(\pi_{1}\right), \mathcal{D}\left(\pi_{2}\right)\right\}, b^{-} \cdot \mathcal{D}\left(\rho_{0}\right) \rrbracket=a^{+} \cdot\left\{\llbracket \mathcal{A}_{1}, \mathcal{C}_{1} \rrbracket, \llbracket \mathcal{A}_{2}, \mathcal{C}_{2} \rrbracket\right\}\left[\Pi / \Pi^{\prime}, \Pi / \Pi^{\prime \prime}, \Delta / \Delta^{\prime \prime}, \Delta / \Delta^{\prime \prime \prime}\right]
$$

where the renamings $\left[\Pi / \Pi^{\prime}, \Pi / \Pi^{\prime \prime}, \Delta / \Delta^{\prime \prime}, \Delta / \Delta^{\prime \prime \prime}\right]$ ensure that the strategy on LHS and the strategy on RHS of the equality are on the same interface $\Pi, \Delta, \sigma^{+}$. We have that

$$
\begin{aligned}
\llbracket \mathcal{D}(\pi), \mathcal{D}(\rho) \rrbracket & =\llbracket a^{+} \cdot\left\{\llbracket \mathcal{A}_{1}, \mathcal{C}_{1} \rrbracket, \llbracket \mathcal{A}_{2}, \mathcal{C}_{2} \rrbracket\right\}\left[\Pi / \Pi^{\prime}, \Pi / \Pi^{\prime \prime}, \Delta / \Delta^{\prime \prime}, \Delta / \Delta^{\prime \prime \prime}\right], \mathcal{B} \rrbracket\left[\Delta / \Delta^{\prime}\right] \\
& =\llbracket a^{+} \cdot\left\{\llbracket \mathcal{A}_{1}, \mathcal{C}_{1} \rrbracket, \llbracket \mathcal{A}_{2}, \mathcal{C}_{2} \rrbracket\right\}, \mathcal{B} \rrbracket\left[\Pi / \Pi^{\prime}, \Pi / \Pi^{\prime \prime}, \Delta / \Delta^{\prime}, \Delta / \Delta^{\prime \prime}, \Delta / \Delta^{\prime \prime \prime}\right] \\
& =\llbracket \mathcal{B}_{0}, \llbracket \mathcal{A}_{1}, \mathcal{C}_{1} \rrbracket, \llbracket \mathcal{A}_{2}, \mathcal{C}_{2} \rrbracket \rrbracket\left[\Pi / \Pi^{\prime}, \Pi / \Pi^{\prime \prime}, \Delta / \Delta^{\prime}, \Delta / \Delta^{\prime \prime}, \Delta / \Delta^{\prime \prime \prime}\right] \\
& =\llbracket \llbracket \mathcal{B}_{0}, \llbracket \mathcal{A}_{1}, \mathcal{C}_{1} \rrbracket \rrbracket, \llbracket \mathcal{A}_{2}, \mathcal{C}_{2} \rrbracket \rrbracket\left[\Pi / \Pi^{\prime}, \Pi / \Pi^{\prime \prime}, \Delta / \Delta^{\prime}, \Delta / \Delta^{\prime \prime}, \Delta / \Delta^{\prime \prime \prime}\right] \\
& =\mathcal{D}(\theta),
\end{aligned}
$$

where the second equality above is justified by the fact that those renamings on "contexts" of visible actions do not modify the calculation of the normal form (and we have correct 
cut-nets on both sides of the equality), the third one by the fact that by construction $a^{+}$ occurs linearly (recall that $\mathcal{B}=a^{-} \cdot \mathcal{B}_{0}$ ) and the fourth one by associativity.

(b) The last rule of $\pi$ is a positive rule $\operatorname{Pos}_{n}$ and $P$ is not principal in $\operatorname{Pos}_{n}$ :

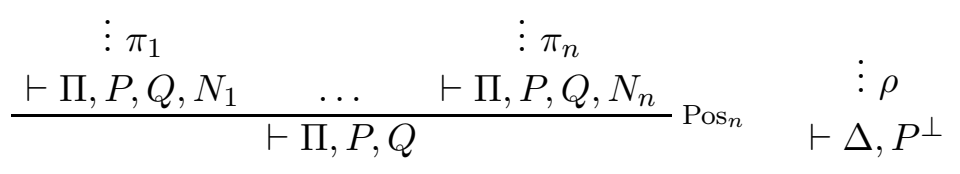

and the principal formula of $\operatorname{Pos}_{n}$ is the occurrence of formula $Q=?_{P}\left(N_{1} \otimes \cdots \otimes N_{n}\right)$.

Let $\mathcal{D}(\pi)=\left(\sigma, I_{n}\right)^{+} .\left\{\mathcal{D}\left(\pi_{1}\right), \ldots, \mathcal{D}\left(\pi_{n}\right)\right\}[\alpha / \sigma]$ be the interpretation of $\pi$ in the sequent of behaviours $\vdash \boldsymbol{\Pi}, \mathbf{P}_{\xi}, \mathbf{Q}_{\alpha}$ on interface $\Pi, \xi^{+}, \alpha^{+}$and $\mathcal{D}(\rho)$ the interpretation of $\rho$ in the sequent of behaviours $\vdash \Delta, \mathbf{P}_{\xi}^{\perp}$ on interface $\Delta, \xi^{-}$.

The procedure described in Theorem A.3 gives a cut-free derivation $\theta$ which is interpreted by $\mathcal{D}(\theta)=\left(\sigma, I_{n}\right)^{+} .\left\{\llbracket \mathcal{D}\left(\pi_{1}\right), \mathcal{D}(\rho) \rrbracket, \ldots, \llbracket \mathcal{D}\left(\pi_{n}\right), \mathcal{D}(\rho) \rrbracket\right\}[\alpha / \sigma]$, where each $\llbracket \mathcal{D}\left(\pi_{i}\right), \mathcal{D}(\rho) \rrbracket$ is the strategy which is interpretation of $\vdash \Pi, Q, \Delta, N_{i}$ on interface $\Pi, \alpha^{+}, \Delta, \sigma i^{-}$.

We have to show that $\llbracket \mathcal{D}(\pi), \mathcal{D}(\rho) \rrbracket=\mathcal{D}(\theta)$, but this easily follows from the definition of normalization, observing that the main strategy $\mathcal{D}(\pi)$ starts with a visible positive action. (c) The last rule of $\pi$ is a negative rule $\mathrm{Neg}_{n}$, having $N=!_{N}\left(P_{1} \not \gamma \ldots \not \gamma P_{n}\right)$ as principal formula.

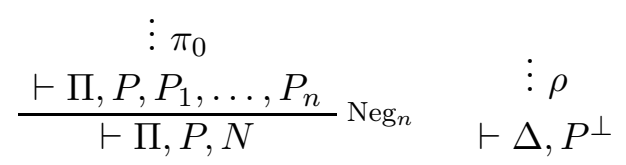

Suppose that $\mathcal{D}(\pi)=\left(\alpha, I_{n}\right)^{-} \cdot \mathcal{D}(\rho)$ interprets $\pi$ in the sequent of behaviours $\vdash \mathbf{\Pi}, \mathbf{P}_{\xi}, \mathbf{N}_{\alpha}$ on interface $\Pi, \xi^{+}, \alpha^{-}$and $\mathcal{D}(\rho)$ interprets $\rho$ in the sequent of behaviours $\vdash \boldsymbol{\Delta}, \mathbf{P}_{\xi}^{\perp}$ on interface $\Delta, \xi^{-}$.

The procedure described in Theorem A.3 gives a cut-free derivation $\theta$ which is interpreted by $\mathcal{D}(\theta)=\left(\alpha, I_{n}\right)^{-} \cdot \llbracket \mathcal{D}\left(\pi_{0}\right), \mathcal{D}(\rho) \rrbracket$, where $\llbracket \mathcal{D}\left(\pi_{0}\right), \mathcal{D}(\rho) \rrbracket$ is the strategy which is interpretation of $\vdash \Pi, P_{1}, \ldots, P_{n}, \Delta$ on interface $\Pi, \alpha 1^{+}, \ldots, \alpha n^{+}, \Delta$.

We have to show that $\llbracket \mathcal{D}(\pi), \mathcal{D}(\rho) \rrbracket=\mathcal{D}(\theta)$, but this easily follows from the definition of normalization, observing that the main strategy $\mathcal{D}(\pi)$ starts with a visible negative action.

We can finally prove:

Theorem B.7 (Soundness). Let $\pi$ be a derivation of a sequent $\vdash \Gamma$ in MELLS and $\mathcal{D}(\pi)$ be the interpretation of $\pi$ in a sequent of behaviours $\vdash \boldsymbol{\Gamma}$.

$$
\mathcal{D}(\pi) \text { is a winning strategy in } \vdash \boldsymbol{\Gamma} \text {. }
$$

Moreover, the interpretation is invariant under cut-elimination.

Proof. If $\pi$ is cut-free, the result is given in Proposition B.3. If $\pi$ contains cuts, then using Lemmas B.1, B.2 and B.4 we can inductively show that $\mathcal{D}(\pi) \in \vdash \boldsymbol{\Gamma}$.

By repeatedly applying Lemma B.6, we eventually prove that $\mathcal{D}(\pi)$ is also the interpretation of a cut-free derivation interpreted in $\vdash \boldsymbol{\Gamma}$. Finally, we use Proposition B.3 again.

This work is licensed under the Creative Commons Attribution-NoDerivs License. To view a copy of this license, visit http://creativecommons.org/licenses/by-nd/2.0/ or send a letter to Creative Commons, 171 Second St, Suite 300, San Francisco, CA 94105, USA, or Eisenacher Strasse 2, 10777 Berlin, Germany 